\title{
Extended TOF-SIMS analysis on \\ low-nickel austenitic stainless steels: \\ The influence of oxide layers on hydrogen embrittlement
}

zur Erlangung des mathematisch-naturwissenschaftlichen Doktorgrades

"Doctor rerum naturalium"

der Georg-August-Universität Göttingen

im Promotionsprogramm ProPhys

der Georg-August University School of Science (GAUSS)

vorgelegt von

Chika Izawa

aus Kanagawa

Göttingen 2015 


\section{Thesis Committee}

Prof. Dr. Astrid Pundt

Institut für Materialphysik, Georg-August-Universität Göttingen

Prof. Dr. Reiner Kirchheim

Institut für Materialphysik, Georg-August-Universität Göttingen

\section{Members of the Examination Board}

Reviewer: Prof. Dr. Astrid Pundt

Institut für Materialphysik, Georg-August-Universität Göttingen

Second Reviewer: . Prof. Dr. Reiner Kirchheim

Institut für Materialphysik, Georg-August-Universität Göttingen

\section{Further members of the Examination Board:}

Prof. Dr. Cynthia A. Volkert

Institut für Materialphysik Georg-August-Universität Göttingen

Prof. Dr. Hans-Ulrich Krebs

Institut für Materialphysik Georg-August-Universität Göttingen

Prof. Dr. Claus Ropers

IV. Physikalisches Institut Georg-August-Universität Göttingen

Prof. Dr. Michael Seibt

IV. Physikalisches Institut Georg-August-Universität Göttingen

Date of the oral examiniation: 21 July 2015 


\section{Contents}

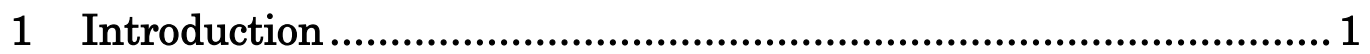

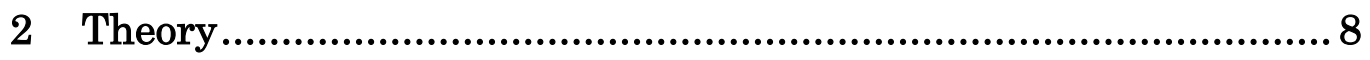

$2.1 \quad$ Austenitic stainless steels............................................... 8

2.1.1 Stainless steels....................................................... 8

2.1.2 Schaeffler diagram...................................................... 10

2.1.3 Martensitic transformation ........................................ 12

2.2 Hydrogen Embrittlement............................................... 14

2.2.1 Hydrogen Embrittlement mechanisms.......................... 16

2.2.2 Tensile test properties and evaluation of the effect of hydrogen on tensile properties.................................... 24

2.2.3 Effect of test variables ............................................... 27

2.2.4 Permeability, diffusivity and solubility of hydrogen ........ 31

2.3 Oxide layer formation ................................................ 38

2.3.1 Oxidation condition - Ellingham diagram .................... 39

2.3.2 Mechanism of oxidation ......................................... 41

2.3.3 Transport mechanism ........................................... 42

2.3.4 Kinetics of oxidation ............................................... 43

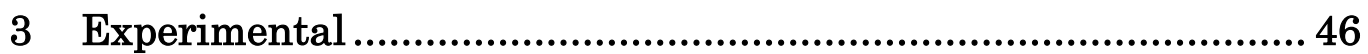

3.1 Alloy Development and evaluation of mechanical properties 46

3.1.1 Chemical composition ............................................ 46

3.1.2 Production of tensile test specimens .......................... 47

3.1.3 Tensile tests........................................................... 48

3.2 Oxide layer analysis........................................................ 49

3.2.1 Secondary Ion Mass Spectrometry (SIMS) .................... 49

3.2.2 SIMS - Sample preparation ........................................ 65

3.2.3 Energy Dispersive X-ray spectrometry (EDX) ...............68

3.3 Dislocation observation and dislocation density measurement68 
3.3.1 Transmission Electron Microscope (TEM) ..................... 68

3.3.2 TEM - Sample preparation......................................... 70

3.3.3 X-ray diffraction $(\mathrm{XRD})$................................................. 71

3.3.4 XRD - Sample preparation ........................................... 74

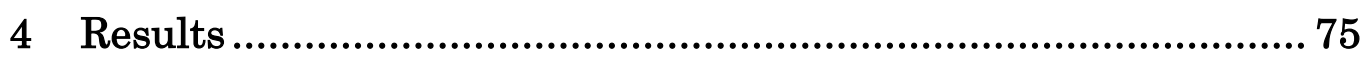

4.1 Selection of furnace ..................................................... 75

4.1.1 Oxide layer formed by three types of furnace................ 75

4.1.2 Influence of annealing time - Inductive heater .............. 78

4.1.3 Influence of alloying elements - Mo heater.................... 80

4.1.4 Selection of furnace.................................................... 83

4.2 Reference steel: Tensile test at RT .................................... 83

4.2.1 Influence of $\alpha^{\prime}$-martensite......................................... 84

4.2.1.1 Tensile test results .............................................. 84

4.2.1.2 SIMS analysis .................................................. 86

4.2.2 Influence of annealing time....................................... 91

4.2.2.1 Tensile test results ............................................... 91

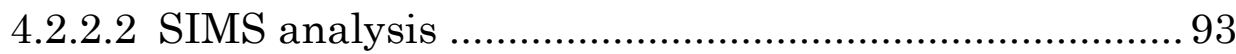

4.2.2.3 Measurement of dislocation density by TEM and XRD .. 96

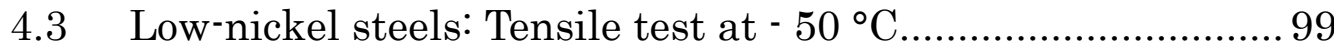

4.3.1 Tensile tests results ................................................ 99

4.3.2 SIMS analysis and EDX ......................................... 102

4.3.3 Dislocation density measured by XRD......................... 110

4.3.4 Martensite formation at $-50{ }^{\circ} \mathrm{C}$ in air ....................... 111

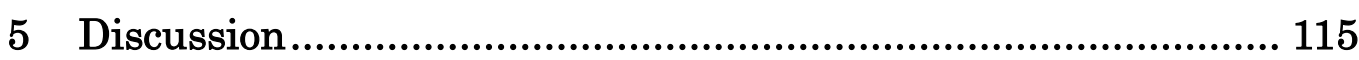

$5.1 \quad$ Oxide layer formation ................................................ 115

5.1.1 Iron (Fe), Chromium (Cr), Nickel (Ni) ....................... 116

5.1.1.1 Stacking sequence of oxide ................................... 116

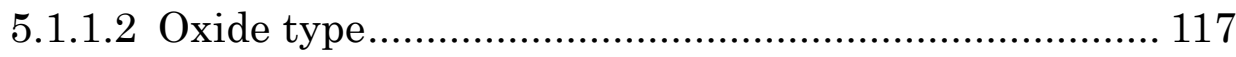

5.1.2 Manganese (Mn) .................................................... 119 


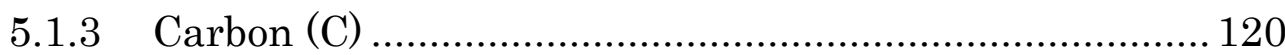

5.1.4 Aluminum (Al) ..................................................... 120

5.1.5 Kinetics of oxidation: details on ion mobilities .............. 121

5.2 Hydrogen embrittlement ............................................... 123

5.2.1 Influence of surface strain-induced a'-martensite and oxide

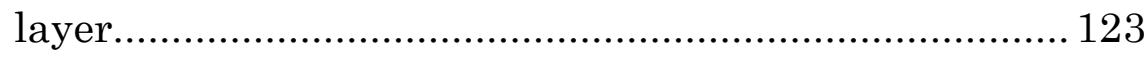

5.2.2 Influence of austenite stability................................. 125

5.2.2.1 Olson-Cohen model: details on $\alpha^{-}$and B-parameters. 126

5.2.2.2 Ni equivalent and $\mathrm{M}_{\mathrm{d} 30}$ temperature...................... 129

5.2.2.3 RRA-value vs $\mathrm{M}_{\mathrm{d} 30}$ at low temperature................... 133

5.2.2.4 RRA-value vs $\mathrm{M}_{\mathrm{d} 30}$ at room temperature ................. 134

5.2.3 Influence of thermal history ..................................... 138

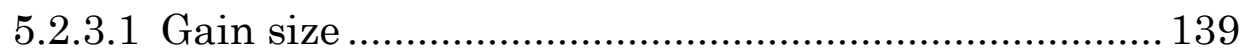

5.2.3.2 Dislocation density and dislocation substructure...... 140

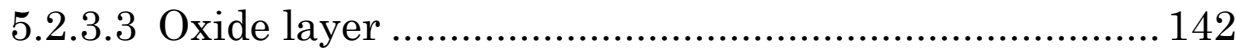

6 Summary ................................................................. 148

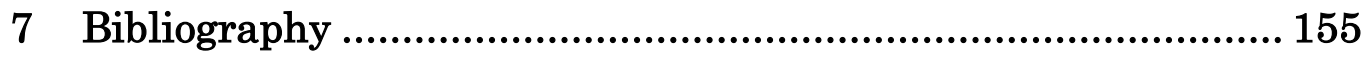

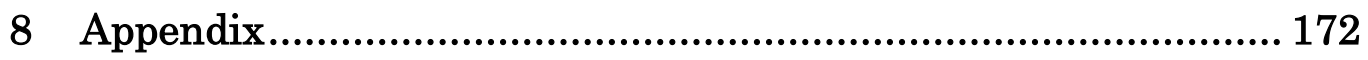

List of publications ........................................................... 182

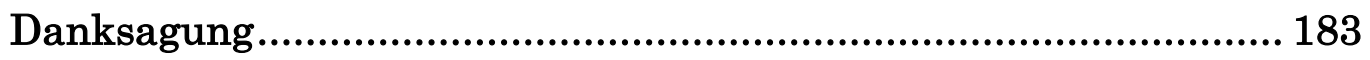

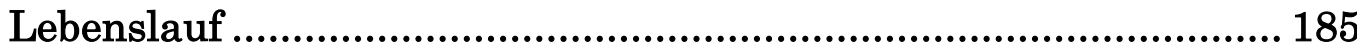





\section{Introduction}

Hydrogen is considered as one of the carbon-free secondary energy carriers. Threfore, automobile industries have developed hydrogen vehicles including fuel cell electricity (FCEVs) and internal combustion engines burning hydrogen (ICEs). In 2009, major automakers stated their goal to commercialize FCEVs from 2015 onwards [1]. The gravimetric energy density of hydrogen, which is around $120 \mathrm{MJ} / \mathrm{kg}$, is more than double that of gasoline while the volumetric energy density of hydrogen is extremely low compared to that of gasoline. In order to achieve performances equivalent with conventional gasoline cars, hydrogen cars need to store hydrogen for a $500 \mathrm{~km}$ range. Based on this, the US Department of Energy's (DOE) target requirements for hydrogen storage system suggest hydrogen gravimetric density of $7.5 \mathrm{wt} \%$ and volumetric density of $70 \mathrm{~g} / \mathrm{L}$, and system fill time for 5 -kg fill of $2.5 \mathrm{~min}$ as an ultimate goal, in 2009 [2].

In order to achieve practical compact hydrogen storage systems, three methods are currently proposed: hydrogen storage materials, high pressure hydrogen gas tanks and cryo-compressed hydrogen tanks [2]. The hydrogen storage materials can reversibly store a considerable amount of hydrogen while they still hold many disadvantages such as gravimetric hydrogen density, reaction kinetics and so on. In addition, many hydrogen storage materials are laboratory base. High pressure and cryo-compressed hydrogen storage systems are currently available and have been tested under real vehicle conditions. For 2015 commercialization, many car manufacturers have decided to use the $70 \mathrm{MPa}$ high pressure carbon composite tank. In the storage system, the gap between the ultimate goal and current status is still large, as shown in Fig. 1.1.

Thus, $70 \mathrm{MPa}$ high pressure carbon composite tank is not the goal for storage systems. Further research and development of storage systems including hydrogen storage materials is required to proceed toward the ultimate goal. 


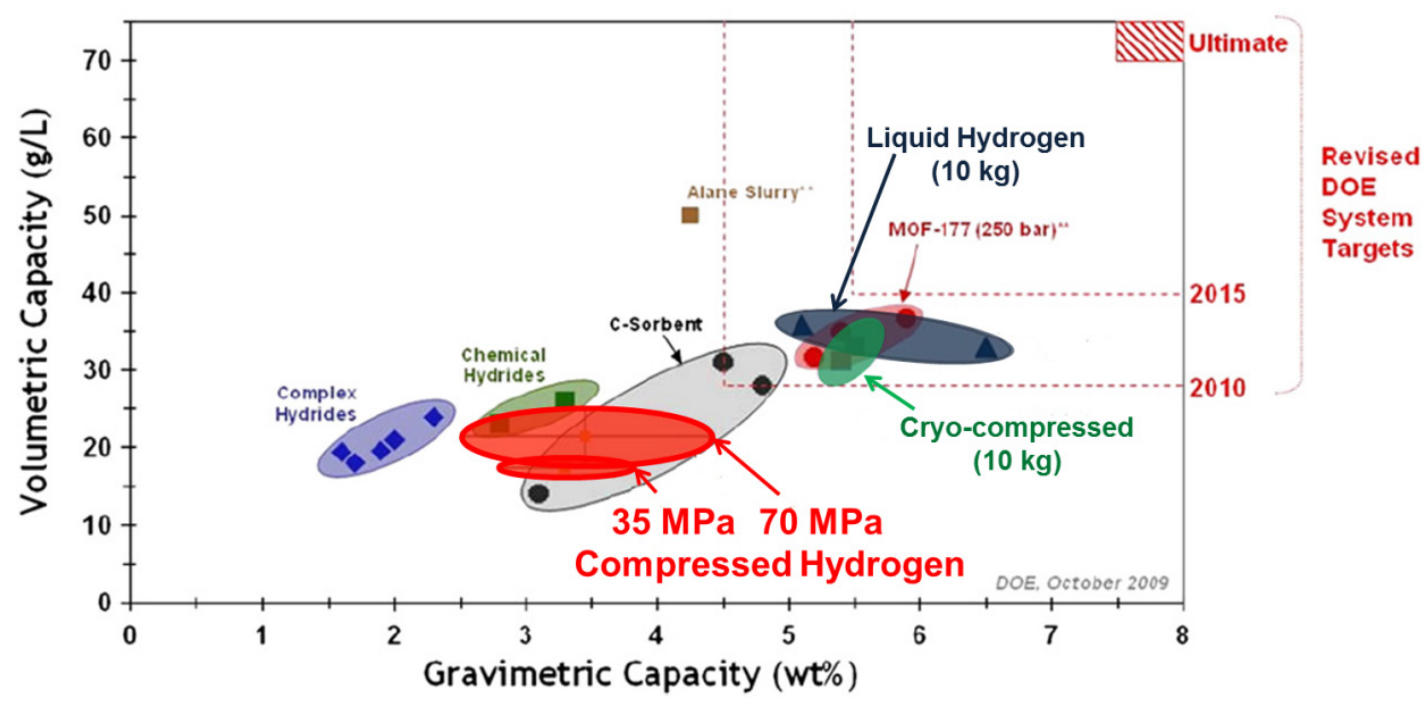

Fig. 1.1 The main storage options and their distance to the DOE storage targets for volumetric and gravimetric capacity. (Figure from Bakker [2])

For the use of hydrogen gas in mobile applications, structural materials such as gas tanks, pipelines, fittings and other components, are directly exposed to high pressure of hydrogen. It is well known that hydrogen degrades materials, causing sudden failure of components [3]. Thus, materials with good mechanical resistance in hydrogen atmosphere are required. Chromium (Cr) -nickel (Ni) austenitic stainless steels are considered as one of the most reliable structural materials and they are potentially used for various components in hydrogen vehicles. They have superior heat and corrosion resistance, good machinability, weld ability and also excellent toughness, - particularly at low, or cryogenic, temperatures.

From the standpoint of material resource conservation and the economy, however, it is desirable to use low $\mathrm{Ni}$ austenitic stainless steels such as AISI 301 and 304. But, low Ni austenitic stainless steels, which are metastable at ambient temperatures, are susceptible to hydrogen degradation.

Hydrogen degradation occurs when hydrogen atoms are accommodated in the materials. The process for hydrogen entry, which might be the rate controlling steps of kinetics, is (1) hydrogen transport, (2) hydrogen adsorption on the surface, (3) dissociation from molecular hydrogen to 
monoatomic hydrogen, (4) hydrogen entry, (5) hydrogen diffusion to the fracture propagation zone in the stress field, and (6) hydrogen trapping at defects such as dislocations, vacancies, grain boundaries, etc. [4]. These steps are schematically illustrated in Fig. 1.2 [3,4].

The hydrogen trapped at defects, especially at dislocations, causes the reduction of the strength, ductility or fracture toughness of a metal and leads to sudden fracture or delayed fracture. This phenomenon is well known as hydrogen embrittlement (HE).

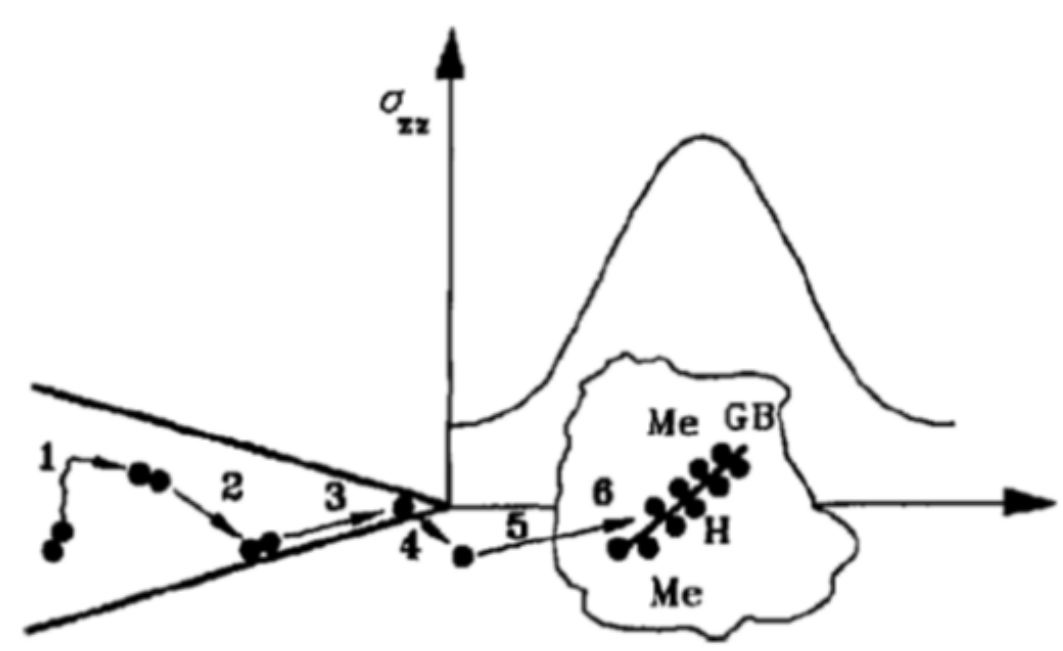

Fig. 1.2 Steps for the transport hydrogen to the fracture propagataion zone [3]. (1) Hydrogen transport, (2) Hydrogen adsorption on the surface, (3) Dissociation from molecular hydrogen to monoatomic hydrogen, (4) Hydrogen entry, (5) Hydrogen diffusion to the fracture propagation zone in the stress field, and (6) Hydrogen trapping at at defects such as dislocations, vacancies, grain boundaries. (Figure from Vehoff [4])

Walter and Chandler [5] and Fukuyama et al. [6] examined HE of austenitic stainless steels at room temperature in $70 \mathrm{MPa}$ hydrogen gas atmosphere and reported that the susceptibility to $\mathrm{HE}$ increased with decreasing Ni content. Many researchers reported on the influence of the austenite stability on $\mathrm{HE}$ since $\mathrm{Ni}$ is a strong austenite stabilizer. They also pointed out that the increased susceptibility to $\mathrm{HE}$ is directly related to a'-martensite, which is partially transformed from austenite during plastic deformation [7-16]. Perng and Altstetter suggested that the localized a' martensite around a crack tip acts as hydrogen diffusion path because of its 
bcc crystal structure [13]. Once hydrogen atoms enter this region, eventually they are trapped in the surrounding austenite grains. As a result, hydrogen concentrates near the crack tip and enhances the crack opening. In fact, hydrogen diffusivity at room temperature in bcc stainless steels is five orders of magnitude higher than that in fcc stainless steels [16]. On the contrary, hydrogen solubility in bcc stainless steel is significantly less than that of fcc stainless steel [16].

However, it is apparent that the transformation to a'-martensite alone cannot explain $\mathrm{HE}$ of austenitc stainless steels because of the materials complexity. Austenite stability can be obtained by a wide variety of chemical compositions and it is known that some austenitic steels are very susceptible to HE [17]. It seems that the role of martensitic transformation is especially relevant for $\mathrm{Cr}-\mathrm{Ni}$ austenitic stainless steels.

In general, the resistance of the stainless steels is proportional to $\mathrm{Ni}$-content. The high resistance of stainless steels with high $\mathrm{Ni}$-content is associated with the phase stability, since $\mathrm{Ni}$ is a strong austenite stabilizer. However, Michler et al. pointed out that there is a large variation in the reported relative reduction of area (RRA), which describes the degree of HE, on similar austenitic stainless steels with low Ni-content [18] as shown in Fig. 1.3. As can be seen in Fig. 1.3, a poor correlation between the RRA-values vs $\mathrm{Ni}$ content is found and the scattering become significant in the range of less than $10 \mathrm{wt} \% \mathrm{Ni}$. This suggests a contribution of the local chemistry in the bulk to HE.

In addition, different thermal histories of the specimen can cause a modification of the surface chemistry. In other words, the heat treatment conditions may affect the composition and thickness of the oxide layer on stainless steels. An understanding of the surface oxidation condition is of considerable importance because the hydrogen entry in metals is fairly sensitive to the surface oxidation condition [19]. But, up to now, such studies have not been reported. Therefore, the surface chemistry of HE-resistant stainless steel samples should be carefully characterized, from both of, application and fundamentals point of view. 


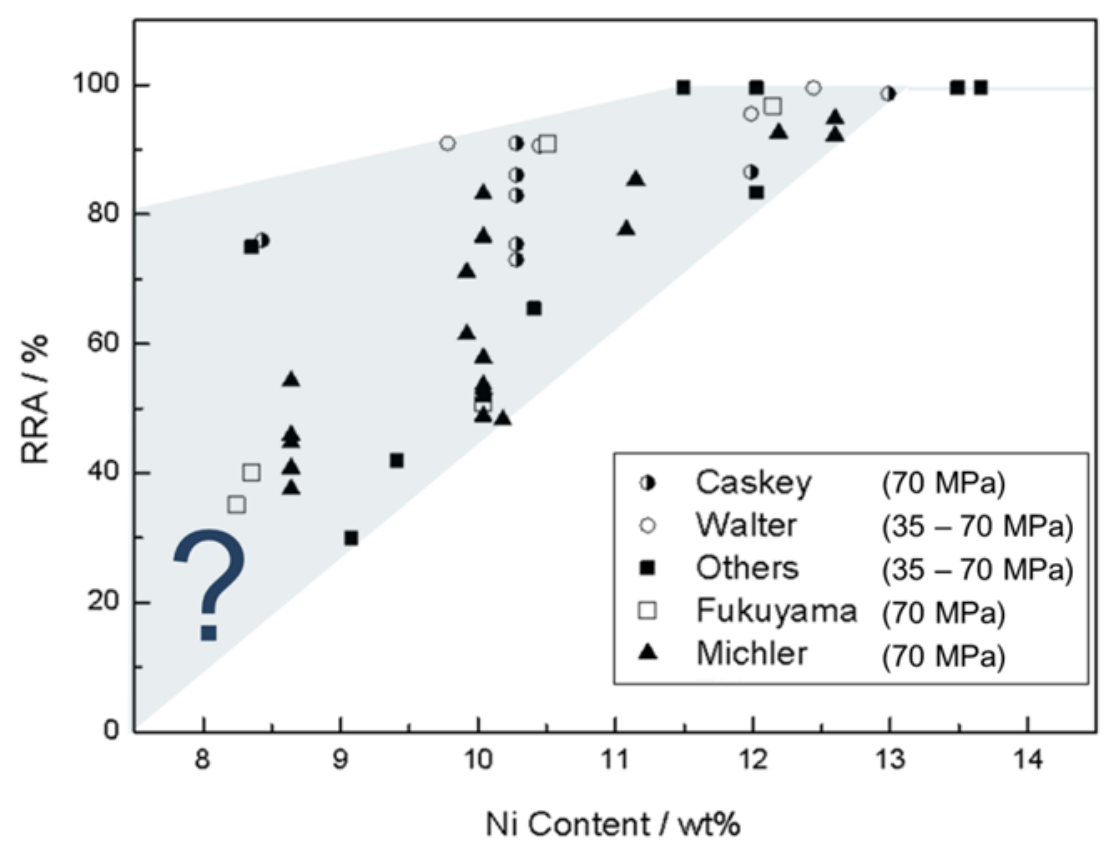

Fig. 1.3 Relative reduction of area (RRA) depending on the $\mathrm{Ni}$ content of the austenitic stainless steel including the results of different research groups [18]. (The figure was replotted.) All tests were performed at room temperature. The scattering in RRA values is considerably large below $10 \mathrm{wt} \% \mathrm{Ni}$.

The aim of this work is to clarify if there is any impact of the surface oxide on the HE accessibility of low-nickel austenitic stainless steels. To determine the oxide layer thickness, the local oxide stacking sequence and the local elemental distribution, the highly sensitive TOF-SIMS method is chosen. To evaluate the contribution of the oxide layer on the HE accessibility, other contributions like a'-martensite content, grain size, dislocation density and dislocation structure have to be considered and studied. The experimental conditions have to be chosen close to the environment of practical hydrogen gas tanks and maximum HE effects are targeted.

This work was performed in collaboration with Bochum university and seven industrial partners ${ }^{1}$. A schematic illustration in Fig. 1.4 provides a

1 This work was financially supported by the Bundesministerium für Wirtschaft und Technologie (BMWi) within the project 0327802C. The division overview of shared roles including the project outputs is schematically represented in Appendix 8.1 
division overview of shared roles allocated to the collaborators, the specimen flow and the type of results in the project.

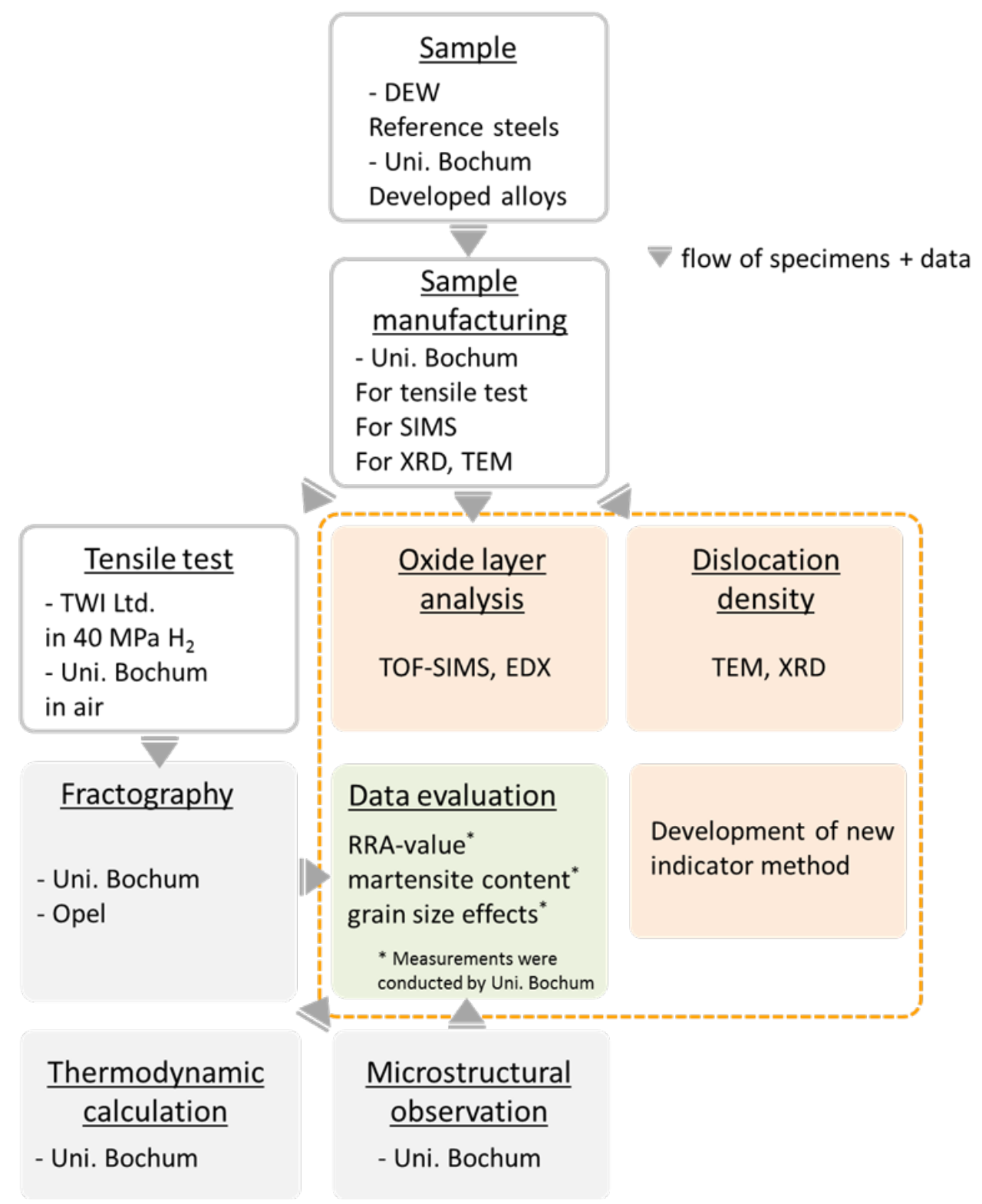

Fig. 1.4 An overview on shared roles allocated to collaborators, specimen flow and type of results

Details on the respective experiments will be described in chapter 3 . In this project, we obtained a wide variety of results, as shown in Fig. 1.4, and divided them into two studies: (1) development of low-nickel austenitic 
stainless steels with a high resistance to HE (published in Ref. [20]), (2) influence of oxide layer type on HE (this study).

The study in Ref. [20] focuses on the development of new low-nickel alloys and includes related results such as thermodynamic calculations for phase diagrams by CALPHAD method, microstructural observations, fractography etc. This study also includes some results published in Ref. [20] and investigates some of them more in detail to propose an alternative indicator to $\mathrm{Ni}$ content for predicting $\mathrm{HE}$.

The research described in this thesis consists of 6 chapters. In chapter 1 , the background and motivation of this study are described. Chapter 2 treats the theoretical background on austenitic stainless steels, hydrogen embrittlement and oxide layer formation. For comprehension, large data collections are provided. In chapter 3, experimental techniques used during this study are introduced. Chapter 4 presents experimental results and their short discussions. Starting with the selection of the suitable furnace in section 4.1, oxide layers formed by three different types of furnaces are characterized. Section 4.2 presents the results obtained on reference stainless steels and on low-nickel stainless steels. Firstly, influences of strain-induced a'-martensite and oxide layer on HE are presented. Then, the influence of the annealing time on HE is shown in order to examine a large scatter in the RRA-values found for the reference samples. Here, results on surface analysis by SIMS, dislocation observations by TEM and dislocation density measurements by XRD on the reference samples are presented. Section 4.2.3 shows the results of low-nickel steels characterized by different techniques including tensile tests, oxide characterizations by SIMS, dislocation densities obtained by XRD and formability of a'-martensite phase. In chapter 5 , all of these results are globally discussed and a general picture on the influence of surface oxides on HE is developed. Chapter 6 summarizes this thesis. 


\section{Theory}

\subsection{Austenitic stainless steels}

\subsubsection{Stainless steels}

Stainless steels are iron-based alloys containing at least $10.5 \mathrm{wt} \%$ chromium. The most remarkable property is their excellent corrosion resistance stemming from the presence of chromium. The chromium in stainless steels forms a protective self-healing chromium rich oxide film, which is called passive film, instantaneously when exposed to oxygen. This layer prevents surface corrosion because of a very low rate of chromium and iron dissolution $[21,22]$.

Stainless steels are categorized as follows by their crystal structures and properties $[23,24]$.

- Austenitic stainless steels are nonmagnetic and their structure is face-centered cubic (fcc). Austenitic stainless steels contain a maximum of $0.15 \mathrm{wt} \%$ carbon, between $16-28 \mathrm{wt} \%$ chromium and sufficient nickel and/or manganese to retain an austenitic structure at all temperatures from the cryogenic region to the melting point of the alloy. They possess excellent ductility, formability, and toughness even at cryogenic temperature. They cannot be hardened by heat treatment, but can be hardened by cold-working.

The properties of austenitic stainless steels can further be modified with the addition of elements such as titanium, copper and molybdenum. Typically modifications in austenitic stainless steel's properties caused by additional elements are summarized in Fig. 2.1 [25]. In the American Iron and Steel Institute (AISI), austenitic stainless steels are covered by the AISI 200 and 300 series. 


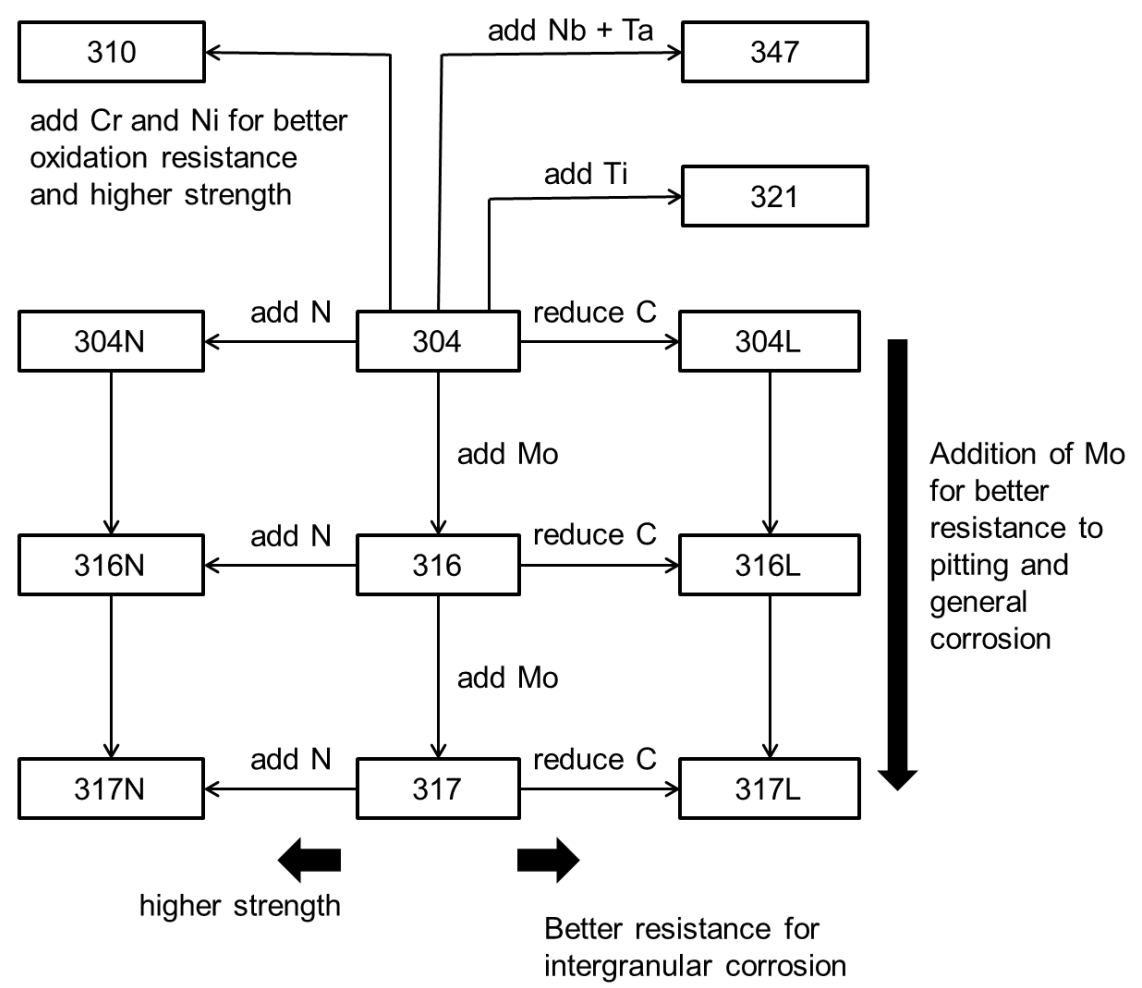

Fig. 2.1 Sketch of modifications of austenitic stainless steels based on an AISI 304 (Fe-18Cr-8Ni). (Figure from Marshall [25])

- Ferritic stainless steels are named after their body-centered-cubic (bcc) structure. These alloys are magnetic. Ferritic stainless steels contain 11-30 wt\% chromium, with only small amounts of the austenite forming element, such as carbon, nitrogen, and nickel. They cannot be hardened by heat treatment. They have good corrosion resistance, particularly to chloride stress corrosion cracking. The AISI designation for these alloys corresponds to 400 series.

- Martensitic stainless steels are magnetic and can be hardened by heat treatment. Their crystal structure is body-centered-tetragonal (bct). They have relatively high carbon content between $0.1-1.2 \mathrm{wt} \%$ and lower amounts of chromium between 12 - 14\%. They are less corrosion resistant than the other counterparts of austenitic and ferritic alloys, because of lower chromium and nickel content. Martensitic stainless steels are mainly used where hardness, 
strength, and wear resistance are required.

- Duplex stainless steels are chromium-nickel-molybdenum alloys which have a mixed microstructure of austenite and ferrite. These alloys are magnetic. The duplex structure results in improved stress corrosion cracking resistance, compared to the austenitic stainless steels, and improve toughness and ductility, compared to the ferritic stainless steels

\subsubsection{Schaeffler diagram}

In order to control mictrostructure and properties, a number of alloying elements are added to the basic alloy as shown in Fig. 2.1. A concept of chromium and nickel equivalents was systematically summarized by Schaeffler [26] to normalize the effect of the alloying elements on phase transformations.

The alloying elements are divided into two groups. Molybdenum (Mo), silicon ( $\mathrm{Si})$, niobium (Nb), titanium (Ti) and aluminum (Al) are ferrite stabilizers. Carbon (C), nitrogen $(\mathrm{N})$, and manganese $(\mathrm{Mn})$ are austenite stabilizers. A nickel equivalent is calculated for the austenite stabilizing elements and a chromium equivalent for the ferrite stabilizing element. The formulae on nickel and chromium equivalents have been modified many times by researchers [27]. In this study, the nickel and chromium equivalents are calculated by the following empirical formulae [20].

$$
\begin{aligned}
\mathrm{Ni}(\text { eq })=\mathrm{Ni}+ & 30(w \mathrm{t} \%[\mathrm{C}+\mathrm{N}])+0.5(w \mathrm{t} \% \mathrm{Mn}) \\
\mathrm{Cr}(\mathrm{eq})=\mathrm{Cr}+ & 1.4(w \mathrm{t} \% \mathrm{Mo})+1.5(w \mathrm{t} \% \mathrm{Si})+05(w \mathrm{t} \% \mathrm{Nb}) \\
+ & 2(w \mathrm{t} \% \mathrm{Ti})+3(w \mathrm{t} \% \mathrm{Al})
\end{aligned}
$$

Plotting the chromium and nickel equivalents on opposing axes provides a graphic depiction of the relationship between composition and microstructure for stainless steels. Fig. 2.2 shows a Schaeffler diagram [28] with main austenitic stainless steels indicated by their AISI codes, which are 


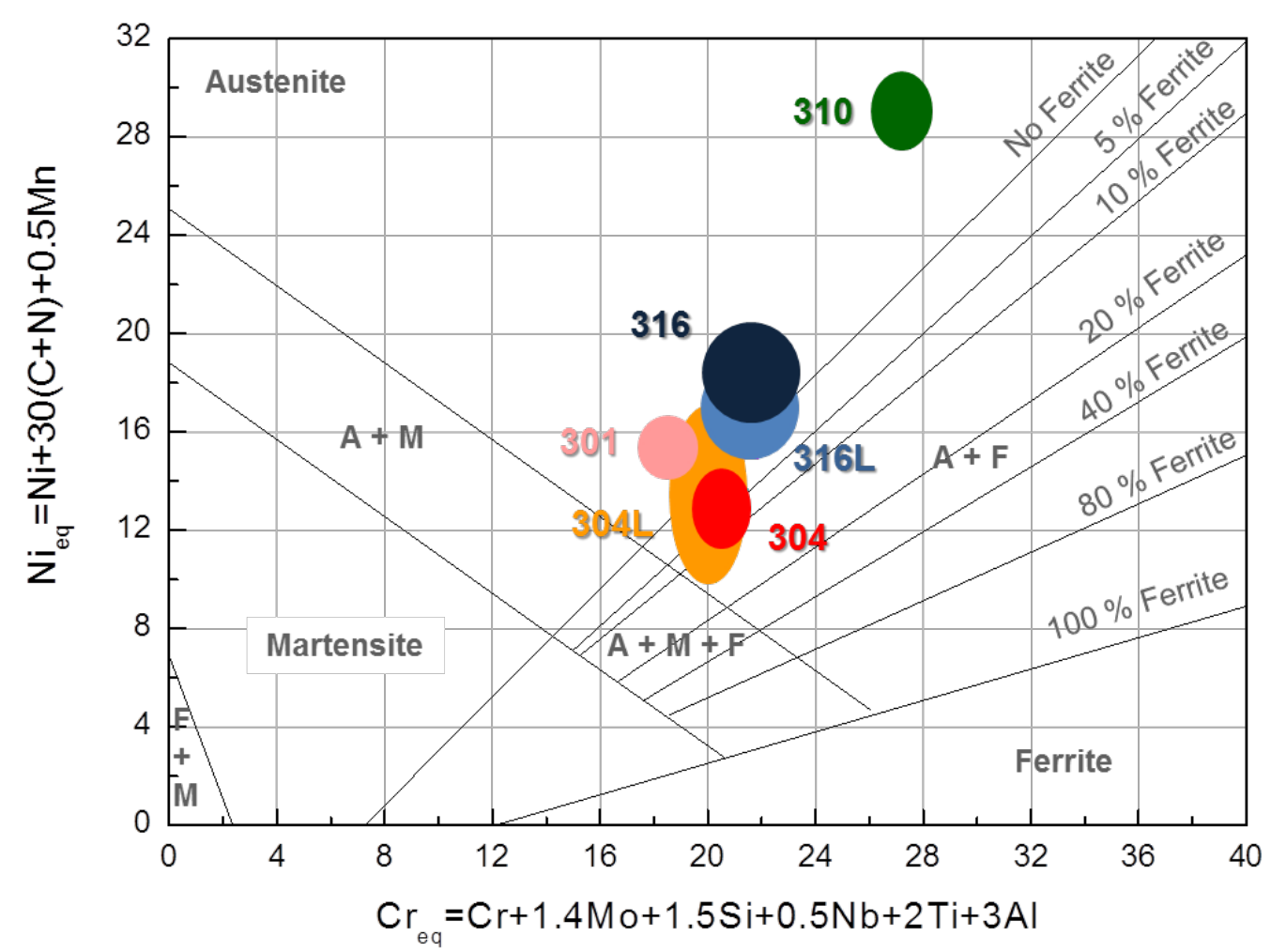

Fig. 2.2 Schaeffler diagram and corresponding main austenitic stainless steels indicated by AISI codes. (Schaeffler diagram from Kikuchi [28])

widely used in industrial applications.

The termed A, M and F in Fig. 2.2 corresponds to Austenite, Ferrite and Martensite, respectively. It should be noted that the Schaeffler diagram does not take into consideration the influence of the cooling rate and aging heat treatments. In Fig. 2.2, the stable stainless steel AISI 310 is found in the upper part of austenite phase and closed to the ferrite phase, while the unstable stainless steels like AISI 301 and 304 fall within, or closed to, the lower part of the austenite region containing a small amount of ferrite.

The Schaeffler diagram had been empirically determined. Any attempts to calculate stability graphs like Schaeffler diagram from thermodynamic basis, quantum mechanical calculations, etc. are less accurate or restricted to ordered systems $[29,30]$. Thus, the Schaeffler diagram is still useful to evaluate austenite stability in various stainless steels and widely referred in both of fundamental and application studies. 


\subsubsection{Martensitic transformation}

Some austenitic stainless steels such as AISI 301, 304, etc. are untastable at ambient conditions and, thus, undergo partial martensitic transformation by rapid cooling (quenching) or by deformation. Two kinds of martensite can form in austenitic stainless steels: a' (bcc) and $\varepsilon$ (hcp).

Many rearchers have reported on a clear impact of a'-martensite phase in austenitic steels to HE since hydrogen diffusivity in a'-martensite is orders of magnitude higher than in the austenite phase (section 2.2.4). Thus $a^{\prime}$-martensite is one of the most problematic factors for $\mathrm{HE}$. In this section, three types of martensitic transformation depending on its origin will be described. An emphasis should be put here, that these martensite phases may be formed in our experiments where tensile tests (deformation) were performed under hydrogen atmosphere at a low temperature of $-50{ }^{\circ} \mathrm{C}$. A detailed review and discussions on the martensitic transformation mechanisms is given by Nishiyama [31].

\subsubsection{Thermally induced-martensite}

Martensite may form instantaneously in austenitic stainless steels during continuous cooling below the martensite start temperature $\left(\mathrm{M}_{\mathrm{s}}\right)$, which is defined as the highest temperature at which the austenite transforms to martensite. This phenomenon is known as athermal martensitic transformation. There are several empirical equations for the $M_{s}$ temperature of steels as a function of their chemical composition [32-34]. Eichelman and Hull's equation [32], which is one of the most widely used, is written as follows

$$
\begin{gathered}
M_{S}\left({ }^{\circ} \mathrm{C}\right)=1302-42(w t \% \mathrm{Cr})-61(w \mathrm{t} \% \mathrm{Ni})-33(\mathrm{wt} \% \mathrm{Mn}) \\
-28(\mathrm{wt} \% \mathrm{Si})-1667[\mathrm{wt} \%(C+N)]
\end{gathered}
$$

This equation suggests that chromium and nickel have a moderate effect on the $\mathrm{M}_{\mathrm{s}}$ compared to very strong effect of carbon and nitrogen. 


\subsubsection{Strain-induced-martensite}

The martensitic transformation can start above the $\mathrm{M}_{\mathrm{s}}$ temperature if deformation of the austenitic stainless steels is given. When the applied stress is sufficiently large to introduce defects such as dislocations and shear bands, this martensitic transformation is said to be strain-induced. There is a temperature about which martensite cannot form under deformation, $\mathrm{M}_{\mathrm{d} 30}$ at which an amount of $50 \%$ austenite will be transformed to martensite through cold-deformation of 0.30 true strain (corresponding ca. 35\% engineering strain). The $\mathrm{M}_{\mathrm{d} 30}$ is determined by the chemical composition, stated in an empirical formula by Angel [35] as follows,

$$
\begin{aligned}
M_{d 30}\left({ }^{\circ} \mathrm{C}\right) & =413-13.7(\mathrm{wt} \% \mathrm{Cr})-9.5(\mathrm{wt} \% N i)-8.1(\mathrm{wt} \% M n) \\
& -18.5(\mathrm{wt} \% M o)-9.2(\mathrm{wt} \% S i)-462(\mathrm{wt} \%[C+N])
\end{aligned}
$$

Grain size has also been reported as an influencing parameter on $\mathrm{M}_{\mathrm{d} 30}$. The large grain size may enhance transformation, indicating that the $M_{d 30}$ increases with increasing grain size [36,37]. Angel's equation was further modified in order to take into account the effect of grain size by Nohara et al. [36]. The equation is as follows,

$$
\begin{aligned}
M_{d 30}\left({ }^{\circ} \mathrm{C}\right) & =551-462[\mathrm{wt} \%(C+N)]-9.2(\mathrm{wt} \% S i)-8.1(\mathrm{wt} \% M n) \\
& -13.7(\mathrm{wt} \% C r)-29(\mathrm{wt} \% N i)-18.5(\mathrm{wt} \% M o) \\
& -29(\mathrm{wt} \% C u)-68(\mathrm{wt} \% N b) \\
& -1.42(\text { grain size number }[\text { ASTM }]-8.0)
\end{aligned}
$$

It should be noted that the larger grain size in $\mu \mathrm{m}$ is represented in the small ASTM grain size number. For instance, the grain size of $50 \mu \mathrm{m}$ and 100 $\mu \mathrm{m}$ is converted to ASTM grain size number of 5.7 and 3.7, respectively. The martensite formation susceptibility and the amount of martensite increase with decreasing the deformation temperature. 


\subsubsection{Hydrogen induced-martensite}

The transformation of austenite to martensite can be also induced in austenitic stainless steels by cathodic charging with hydrogen. A number of researchers reported the formation of hcp $\varepsilon$ hydride and fcc $\mathrm{Y}^{*}$ hydride in 300 series stainless steels as a result of cathodic hydrogen charging [38-44]. In the unstable stainless steels like AISI 304, they transforms to hydrogen free phases of $\alpha^{\prime} \mathrm{H}$ and $\varepsilon_{\mathrm{H}}$, upon outgassing of hydrogen after charging. These phases are often called hydrogen induced phases. In the case of stable austenitic stainless steels like 310 stainless steels, such a' martensitic transformation is not detected [39].

The $\varepsilon$ martensitic transformation can be also induced by high pressure gaseous charging. Narita et al. [39] reported that the small $\varepsilon$ reflections were observed by X-ray diffraction measurements of AISI 304 and 310s hydrogenenated at $300{ }^{\circ} \mathrm{C}$ under $70 \mathrm{MPa}$ hydrogen gas pressure. In the case of AISI 304, the $\varepsilon^{-r e f l e c t i o n s ~ d i s a p p e a r e d ~ a n d ~ t h e ~ a ~ r e f l e c t i o n s ~ a p p e a r e d ~}$ after aging treatment of $150 \mathrm{~min}$ at $293 \mathrm{~K}$ [39]. Hoelzel et al. presented that the $\varepsilon$ reflections were observed in AISI 304 charged at $350{ }^{\circ} \mathrm{C}$ in hydrogen at 3.0 GPa [45]. Below this pressure, the $\varepsilon$-martensite reflections were not observed [45].

\subsection{Hydrogen Embrittlement}

In this section, a term named HE (Hydrogen Embrittlement) and its categorization will be described based on some phenomenological aspects of HE discussed in the history, since recently-published papers often refer also to "Hydrogen Environment Embrittlement (HEE)" as a sub-category of the HE. After that, we move on to see the complexity of HE from the viewpoint of fractographic features. By this, we will notice the reason why HE has a long history of diverse studies, leading to many mechanisms proposed so far. These HE mechanisms will be briefly surveyed in section 2.2.1,

Hydrogen embrittlement (HE) refers to a phenomenon that hydrogen causes the reduction of the strength, ductility or fracture toughness of a 
metal and leads to sudden fracture or delayed fracture [46]. HE can be categorized into either "Internal Hydrogen Embrittlement (IHE)" or "Hydrogen Environment Embrittlement (HEE)" by whether hydrogen is present originally in the metal lattice or originates in the environment surrounding the metal [47]. In other words, IHE may occur due to the presence of hydrogen introduced during the manufacturing process such as steel making, pickling and electroplating while HEE may occur when a hydrogen free material is under mechanical stresses in hydrogen environment. This thesis focuses on HEE. Therefore, the tensile tests were conducted on specimens at $40 \mathrm{MPa}$ hydrogen pressure. Thus, henceforth, HE mainly means HEE in this study.

During the last few decades, many mechanisms have been proposed to explain the HE. However, there is no clear consensus on one single theoretical mechanism. The HE process varies from material to material and strongly depends on the experimental conditions such as tensile test temperature or the environmental gas pressure. Some typical failure modes of austenitic stainless steels tensile tested in $\mathrm{H}_{2}$ are shown as fractographies in Fig. 2.3 [20].

As shown in Fig. 2.3 (a), dimple rupture pattern is observed on AISI 316L tested at room temperature. This fracture mode indicates a ductile fracture. This type of fracture is often observed on very stable austenitic stainless steels such as AISI 310 and 316 [20]. Intergranular fracture is observed on the high carbon and high nitrogen containing steel, tested at room temperature, as shown in Fig. 2.3 (b). This fracture mode proceeds along the grain boundaries. This is caused by impurity segregation and/ or a continuous carbide phase and is characterized as a brittle fracture [48]. The fracture occurs also at twin boundaries. Twin-boundary parting found on AISI 304 tested at $-50{ }^{\circ} \mathrm{C}$ is shown in Fig. 2.3 (c). This twin-boundary parting is commonly observed on AISI 304 tested at low temperatures, i.e. -73 to $-23{ }^{\circ} \mathrm{C}$ and is characterized by single facets extending over one grain $[20,48]$. In Fig. 2.3 (d), transgranular cleavage appears for AISI304, tested at room temperature. Transgranular cleavage is the typical mode for ferritic and 
martensitic stainless steels. However, it is also observed in AISI 304 which contains ferric and/or martensite phase and propagation the cleavage proceeds along the interfaces of austenite/martensite or austenite/ferrite $[20,48]$.

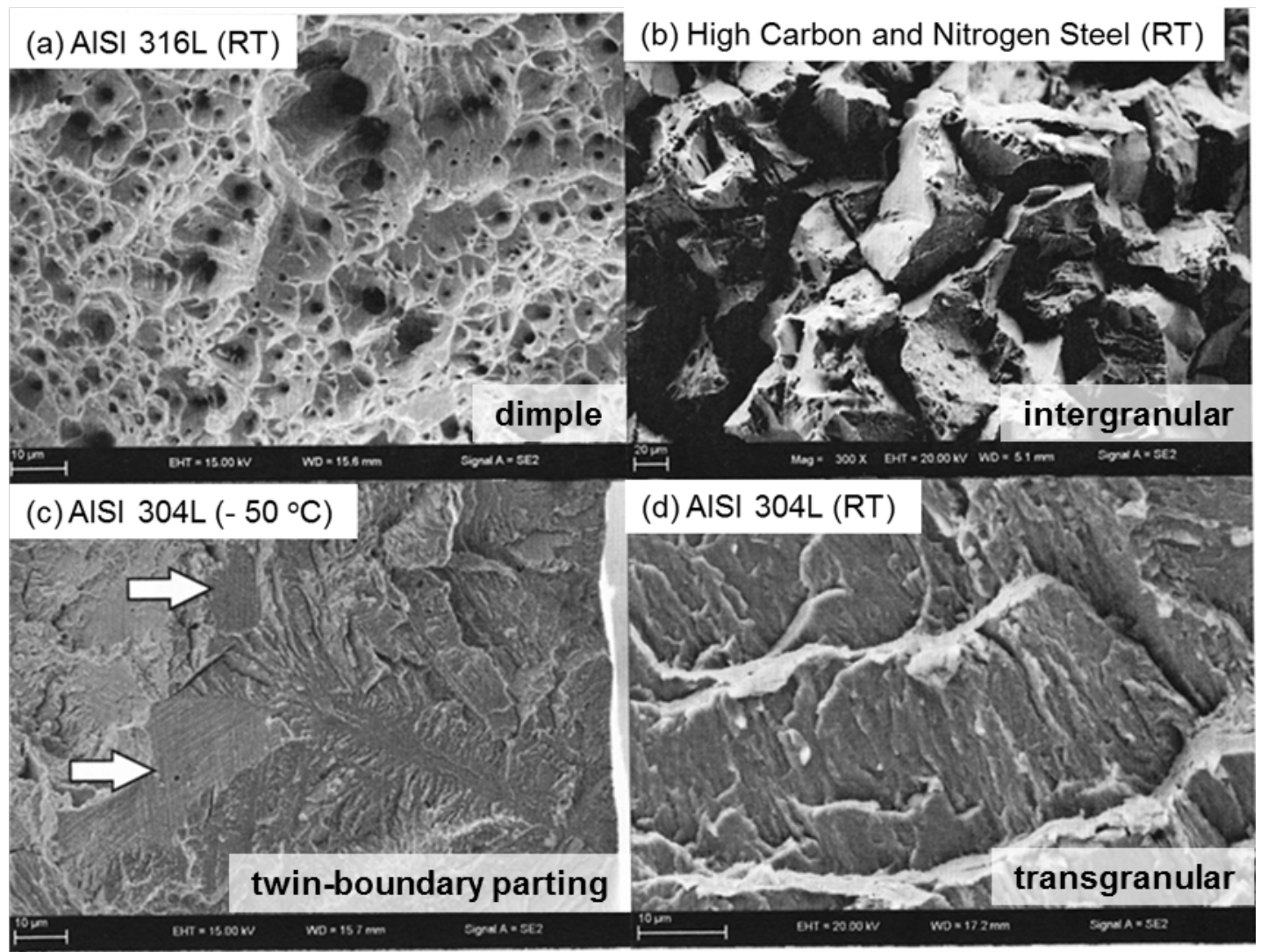

Fig. 2.3 Typical fracture modes of austenitic stainless steels tested in $\mathrm{H}_{2}$ [20].

(a) AISI 316 at RT: dimple rupture, (b) High carbon and high nitrogen steel at RT: intergranular, (c) AISI 304 at $-50{ }^{\circ} \mathrm{C}$ : twin-boundary parting indicated by arrows and (d) AISI304 at RT transgranular cleavage. Different fracture modes are observed depending on materials and test temperature (Picture from Martín [20], with permission of the author)

As introduced above, $\mathrm{HE}$ in austenitic stainless steels may exhibit a fracture surface of cleavage, of intergranular or of transgranular fracture. That is, no single fracture mode is characteristic for $\mathrm{HE}$ and thus the explanations on HE-mechanisms become rather complex as described in the next section.

\subsubsection{Hydrogen Embrittlement mechanisms}

As described in the previous section, there is no clear consensus on a 
single theoretical mechanism to explain all effects of HE. In the following, we briefly address four candidate mechanisms. They are often named as hydrogen assisted cracking (HAC), hydrogen enhanced decohesion (HEDE), adsorption-induced dislocation emission (AIDE) and hydrogen enhanced localized plasticity (HELP). A novel concept called DEFectACTingAgeNT (Defactant) theory proposed by Kirchheim is also introduced [49]. Further details including other mechanisms such as internal pressure theory, surface energy contributions etc. can be found elsewhere [50-53].

\subsubsection{Hydrogen Assisted Cracking (HAC)}

Hydrogen Assisted Cracking (HAC) was proposed by Beachem in 1972 [54]. This model is based on the observation of the lowering of the torsion flow stress in hydrogen charged AISI 1020 steel, suggesting that the presence of sufficiently concentrated hydrogen dissolved in the lattice just ahead of the crack tip aids deformation processes.

A remarkable point of this HAC mechanism is that the interaction between hydrogen and dislocation is mentioned. It means that hydrogen allows dislocations to multiply or move at reduced stress. Beachem's model had not been widely accepted since this idea didn't accommodate to the conventional "embrittlement" theories of the period. However, the concept of HAC was later revisited into Adsorption Induced Dislocation Emission (AIDE) mechanism proposed by Lynch in 1976 [55]. Further, the idea of solute hydrogen facilitating dislocation motion was subsequently promoted (from the 1980s onwards) by Birnbaum, who proposed the Hydrogen Enhanced Localized Plasticity (HELP) mechanism [56].

\subsubsection{Hydrogen Enhanced Decohesion (HEDE)}

Hydrogen enhanced decohesion (HEDE) was first proposed by Troiano [57-59] and further developed in detail by Oriani et al. [60-63] This mechanism postulates that the cohesive strength of the lattice decreases with increasing hydrogen concentrations in lattice. From this, one can expect 
that large concentration of hydrogen should be needed. However, when hydrogen accumulates only in the region ahead of the crack tip where the tensile stresses are maximized, the sufficient hydrogen concentration locally attains. Then hydrogen reduces the cohesive bonding strength between metal atoms and cleavage of these bonds occurs. Thus, weakening of the cohesive force between bonds would permit nucleation of microcracks.

Oriani et al. determined the plane-strain stress intensity factor $\left(\mathrm{K}_{t h}\right)$ as a function of the threshold pressure $(\mathrm{P})$, of hydrogen gases necessary to cause crack opening in AISI 4340 steel of $1700 \mathrm{MPa}$ yield strength at room temperature [62]. The theoretical calculation based on the $\mathrm{K}_{t h}{ }^{-} \mathrm{P}$ relation results in a local hydrogen concentration at the region ahead of the crack tip that is $10^{3}-10^{4}$ times higher than the average concentration of hydrogen [64]. The HEDE mechanism remains a matter of debate since direct experimental observation of atomic-scale events at crack tips in bulk specimens is currently lacking.

\subsubsection{Adsorption Induced Dislocation Emission (AIDE)}

The concept of HAC was reintroduced by Lynch [55], who later proposed Adsorption Induced Dislocation Emission (AIDE) mechanism. He pointed out the similarities between HAC and Liquid Metal Embrittlement (LME) through metallographic and fractographic observations for HAC and LME in the same materials (Fe-2.6\% Si single crystal, aluminum alloys, nickel single crystals, high strength steels, magnesium) [55].

Fig. 2.4 shows the fracture surface produced in a nickel single crystal with a near $<100>$ orientation by rapid crack growth in liquid mercury and then rapid growth in gaseous hydrogen [55]. Tear ridges, isolated large dimples and slip lines were found in hydrogen and mercury environments. LME during rapid fracture is due to adsorbed mercury since liquid metal cannot diffuse into the bulk. Thus, the similarities in fracture feature between HAC and LME suggest hydrogen adsorption could be the rate 
controlling step in the fracture process and are responsible for $\mathrm{HAC}$ in the same way as adsorbed metal atoms are responsible for LME.

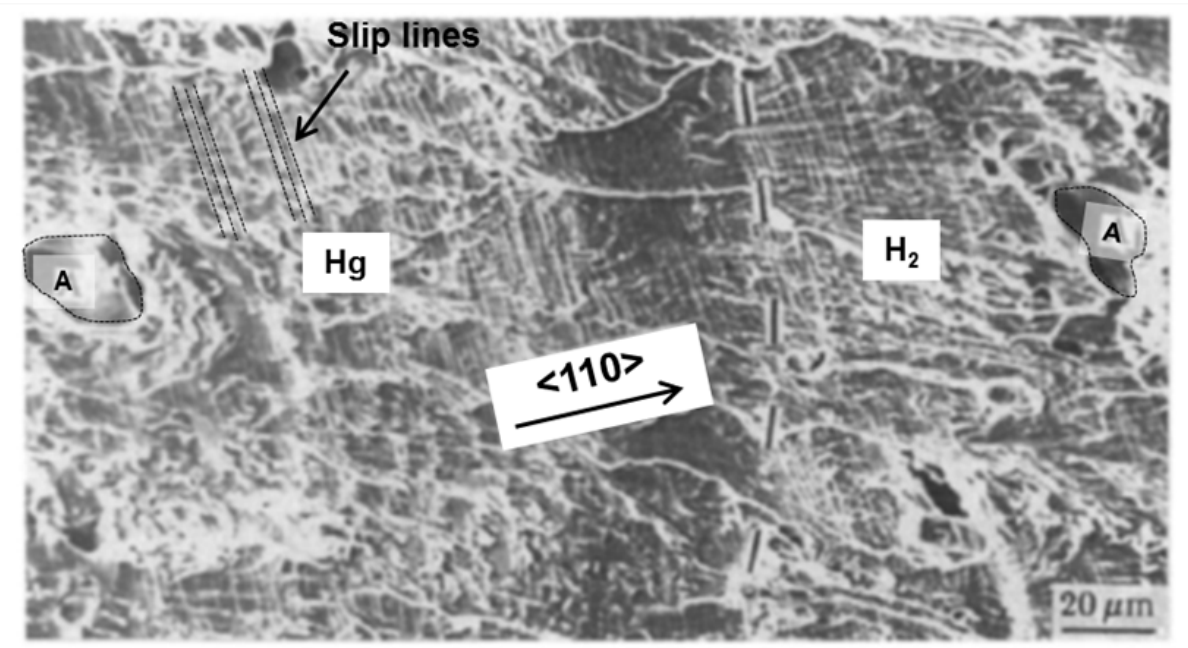

Fig. 2.4 Fracture surface produced in Ni single crystal with a $<100>$ orientation by rapid crack growth $(\sim 1 \mathrm{~mm} / \mathrm{s})$ in liquid mercury and then rapid crack growth $(\sim 1$ $\mathrm{mm} / \mathrm{s})$ in hydrogen $(\sim 101 \mathrm{kPa})$ at $20^{\circ} \mathrm{C}$. Tear ridges, isolated large dimples $(A)$, and slip lines (partialy indicated by dotted lines) were found in both cases. (Picture from Lynch [55])

On the basis of these observations, the AIDE mechanism was proposed. In the AIDE model, adsorbed hydrogen atoms weaken interatomic bonds at crack tips and thereby facilitate the nucleation of dislocations from crack tips. Once nucleated, dislocation can readily move away from the crack tip first on e.g. plane A and then on plane B and so forth, producing an increment of crack advance, $\Delta \mathrm{a}$ (Fig. 2.5). Under sustained or monotonically increasing stresses, crack growth occurs not only by dislocation-emission from crack tips, but also involves nucleation and growth of microvoids (or nano-voids) at second-phase particles, slip band intersection, or other site in the plastic zone. The coalescence of cracks growing by alternate-slip with voids re-sharpens crack tips. However, the crack growth occurs primarily by dislocation emission from crack tips [52]. 


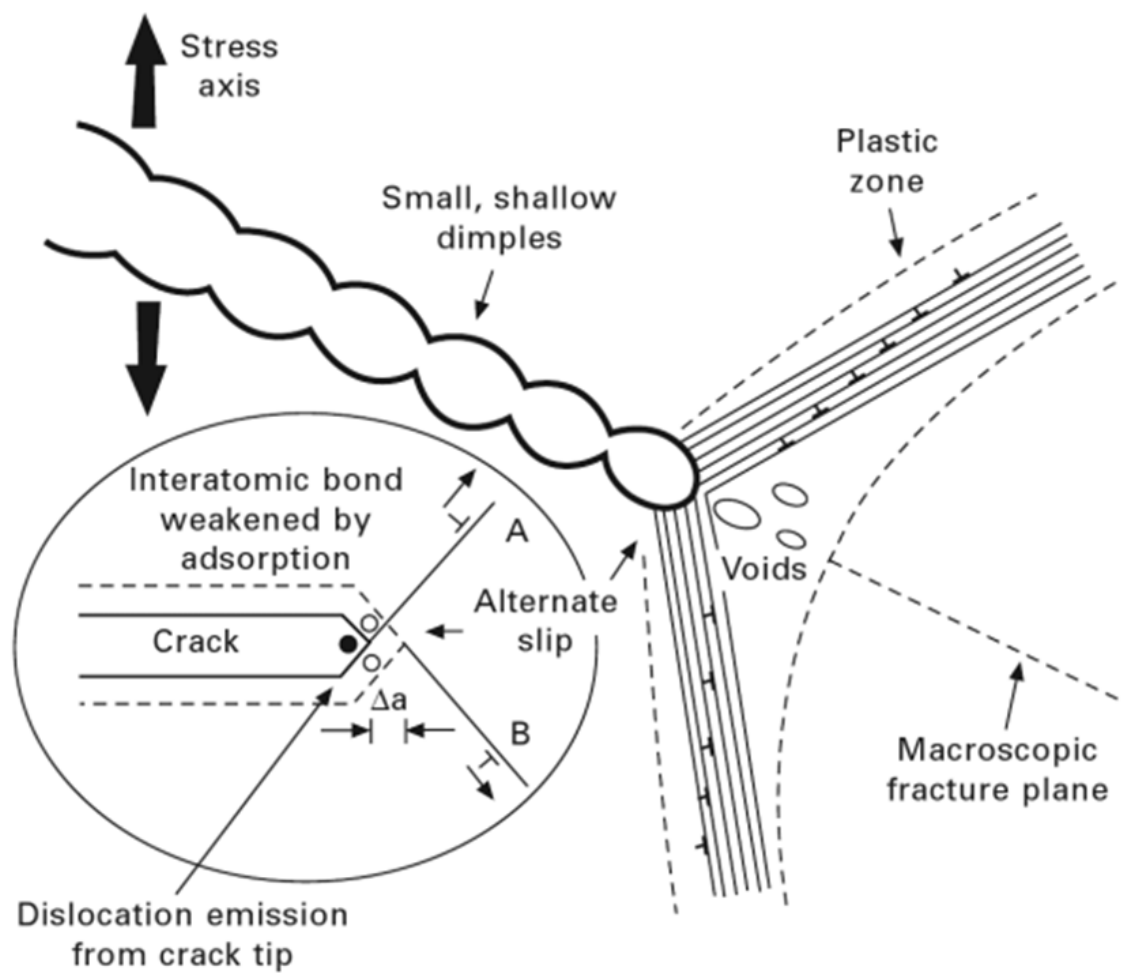

Fig. 2.5 Schematic diagrams illustrating AIDE mechanism [52]. Adsorbed hydrogen atoms weaken interatomic bonds at crack tips and thereby facilitate the nucleation of dislocations from crack tips. Dislocation emission from crack tip first on plane $A$ and then on plane $B$ produces an increment of crack advance, $\Delta a$. The coalescence of cracks growing by alternate-slip with voids in the plastic zone re-sharpens crack tips (Picture from Lynch [52])

\subsubsection{Hydrogen Enhanced Localized Plasticity}

\section{(HELP)}

Hydrogen Enhanced Localized Plasticity (HELP) mechanism was proposed by Birnbaum, Sofronis, Robertson, and co-workers [56,65-72]. As described in 2.2.1.1, the HELP mechanism evolved from the HAC mechanism. A requirement for the HELP process is the accumulation of hydrogen in the vicinity of the crack tip or in the stress area of dislocations. During the initiation of a dislocation movement by external stresses, hydrogen enhances dislocation movement at lower stresses. Thus hydrogen assists the plastic deformation and leads local ductile failure with a brittle appearance on the macroscopic scale.

Tabata and Birnbaum [73] performed in situ environmental TEM observations of thinned Fe specimens. During the observations, the 
specimens were subjected to plastic deformation in either vacuum or hydrogen gas. Their results revealed that dislocation nucleation facilitated at ahead of crack tips in the hydrogen gaseous environment and cracks propagated by the emission of dislocations from the crack tips into the solid. Further, the plastic processes accelerated and the crack tip increased its width and its velocity increased with increasing hydrogen gas pressure in environmental cell. [73].

Direct observations of hydrogen effects on dislocations have been carried out for bcc, fcc, and hcp, metals [65,67-70,72,74]. In all of the systems, hydrogen increased the dislocation mobility under constant stress conditions. This behaviour was observed for edge, screw and mixed dislocations and for isolated dislocations as well as dislocation tangles [56].

The effect of hydrogen on dislocation mobility suggests that it derives from the elastic interactions between dislocations and barriers to their motion. Sofronis and Birnbaum calculated the effects of $\mathrm{H}$ on the elastic interactions between defects using linear elasticity and finite element techniques [75]. They used the parameters for the calculations of hydrogen effects on the dislocation interactions since there is a full set of data available for the $\mathrm{Nb}-\mathrm{H}$ system. The calculated distribution of hydrogen with the stress field of a single edge dislocation is symmetric with respect to the dislocation plane. Under the same temperature and nominal hydrogen concentrations, hydrogen atmospheres around two edge dislocations on the same slip system and with the same Burgers vectors is non-symmetric, as shown in Fig. 2.6 [75].

For dislocations of the same sign, the hydrostatic stress field is reinforced positively below the slip plane and negatively above the slip plane (Fig. 2.6 (a)) [75]. This reinforcement increases as the dislocations approach each other. For dislocations of the opposite sign, the positive hydrostatic stress field of each dislocation is weakened, as shown in Fig. 2.6 (b) [75]. 
(a)

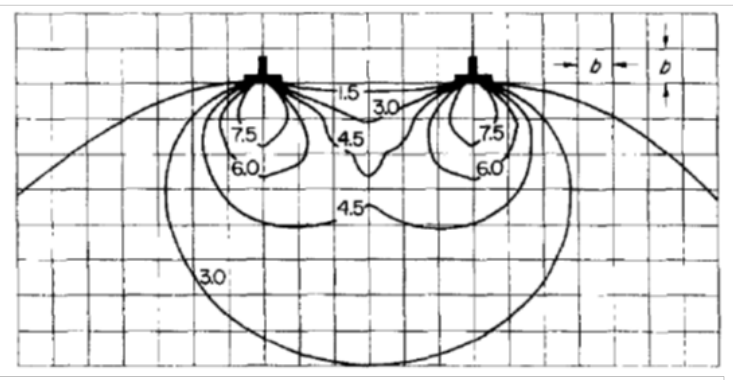

(b)

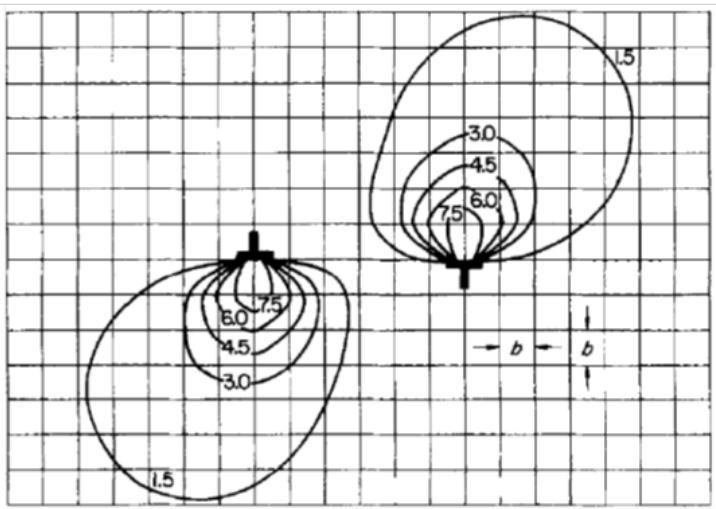

Fig. 2.6 Isoconcentration contours of the normalized hydrogen concentration, $\mathrm{C} / \mathrm{C}_{0}$, around two parallel edge dislocations having (a) equal and (b) opposite Burgers vectors of magnitude, $b$, on the same slip plane at a separation distance of 6b. Calculations are for the parameters of $\mathrm{Nb}$ containing $\mathrm{C}_{0}=0.1(\mathrm{H} / \mathrm{M}=0.1)$ at 300 K. (Figure from Sofronis [75])

In general, two parallel edge dislocations on the same slip system and with Burgers vectors of the same sign repel each other. Their finite element calculation of $\mathrm{H}$ shielding indicate that the shielding greatly reduces this repulsive force acting between the dislocations on the glide plane [56].

\subsubsection{Defactant Concept}

The defactant concept is a thermodynamic approach for HE of metals proposed by Kirchheim [76]. This model is based on the behavior of surfactants (SURFace ACTing AgeNTS) in liquids [76]. The effect of surfactants in liquids reduces the surface energy and is described by the Gibbs adsorption equation [77]

$$
\left.\frac{\partial \gamma}{\partial \mu_{A}}\right|_{T, n_{B}, a}=-\Gamma_{A}
$$

where $\mu_{A}$ is the chemical potential of solute $A$ being segregated with an excess $\Gamma_{A}$ at the surface with an area $a$ in a material $\mathrm{B}$ of $n_{B}$ atoms or molecules [77]. This equation is also valid for other crystalline defects interacting with hydrogen [76,78], and the new term defactants (DEFect ACTing AgeNTS) was introduced by Kirchheim [76]. 
Now, we consider the hydrogen-dislocation interaction in metals with the defactant concept. In this case, the Gibbs adsorption isotherm of Eq.(2 6) is rewritten as: [76]

$$
\left.\frac{\partial \gamma_{d i s}}{\partial \mu_{H}}\right|_{V, T, l_{d i s}, n}=-\Gamma_{H}^{d i s}
$$

where the quantity $\gamma_{\text {dis }}$ is defined as the dislocation line energy. In a partially closed system like Wagner's Gedanken experiment, an excess hydrogen per dislocation length $\Gamma_{H}^{d i s}$ can be defined as: [76]

$$
\left.\Gamma_{H}^{d i s} \equiv \frac{1}{V} \frac{\partial n_{H}}{\partial l_{d i s}}\right|_{V, T, \mu_{H}, n}
$$

Equation (2-8) states that an incremental change of the dislocation length $l_{d i s}$ causes an incremental change of the number of hydrogen atoms (defactants) $n_{H}$ in a system of constant volume $V$, constant temperature $T$, constant chemical potential of hydrogen $\mu_{H}$, and number of metal atoms $n$. Thus, the excess of hydrogen $\Gamma_{H}^{d i s}$ is positive, if $n_{H}$ increases with increasing $l_{\text {dis }}$.

The excess of hydrogen (defactants), given by Eq.(2-7), is positive and reduces the dislocation line energy via Eq. (2-8), similar to the reduction of surface energy by surfactants in liquids.

The defactant concept gives a more general explanation for parts of the HELP mechanism (2.2.1.4). In terms of the defactant concept, the line energy of dislocations is reduced in the presence of hydrogen. Consequently, dislocation sources become more active, and the newly generated dislocations push former dislocations. Further, if hydrogen segregating (defactants) to kinks reduce the kink formation energy, the enhanced dislocation motion occurs by the ease of kink pairs formation. TEM observations of the dislocation motion in hydrogen atmosphere [69] reflect these processes. A more detailed discussion concerning the defactant concept, including the derivation of equations and experimental evidence is found in Ref. [49,76-78] 


\subsubsection{Tensile test properties and evaluation of the effect of hydrogen on tensile properties}

The susceptibility of materials to $\mathrm{HE}$ is frequently evaluated by comparison of tensile tests performed in hydrogen gas and in an inert environment such as air and helium. These tensile tests have been performed on all samples and the results could be used for interpretations.

Tensile test provides a basic information on the strength and ductility of materials. Stress-strain curves obtained by the tensile test show the response of a material to an applied stress. This curve can be expressed as either engineering or as true stress-strain curve. For many engineering applications, the use of engineering stress and strain values will be sufficient for a plasticity analysis of materials. Engineering stress $\left(\sigma_{E}\right)$ and engineering strain (e), which are also called as nominal stress and nominal strain, respectively, are expressed as follows,

$$
\begin{array}{ll}
\text { Engineering (nominal) stress: } & \sigma_{E}=\frac{P}{A_{0}} \\
\text { Engineering (nominal) strain: } & \mathrm{e}=\frac{\Delta l}{l_{0}}=\frac{\left(l-l_{0}\right)}{l_{0}}
\end{array}
$$

where $P$ is the load, $A_{0}$ is the original cross sectional area, $\Delta l$ is the gage length and $l_{0}$ is the original gage length.

True stess $\left(\sigma_{T}\right)$ and strain $(\varepsilon)$ are applied when dimension change during a tensile test is taken into account. True strain and true strain are given by,

$$
\begin{array}{ll}
\text { True stress: } & \sigma_{T}=\frac{P}{A_{i}}=\sigma_{E}(1+e) \\
\text { True strain: } & \varepsilon=\int_{l_{0}}^{l} \frac{d l}{l_{0}}=\ln \frac{l}{l_{0}}=\ln (1+\mathrm{e})
\end{array}
$$

where $A_{i}$ is the instantaneous cross sectional area and e is engineering strain.

A schematic engineering stress-strain diagram is shown in Fig. 2.7. The 
major parameters provided from stress-strain curve obtained during the tensile test are percentage elongation $(\Delta l \%)$, the reduction of area (RA \%), the yield strength $\left(\sigma_{y}\right)$ or $0.2 \%$ offset yield strength $\left(\sigma_{0.2 \%}\right)$, ultimate tensile strength $\left(\sigma_{U T S}\right)$, and fracture strain $\left(\varepsilon_{f}\right)$.

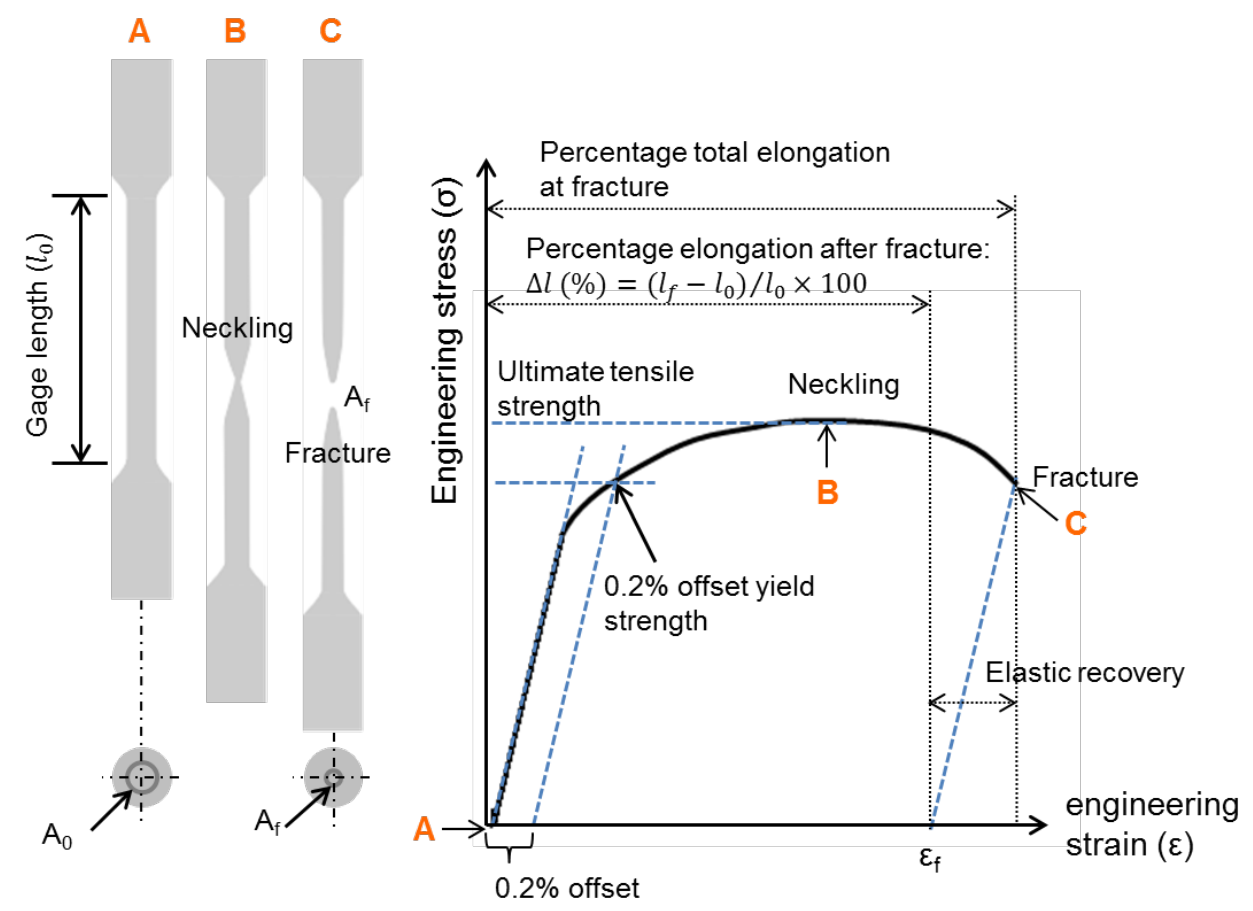

Fig. 2.7 Stress-strain curve. Percentage elongation $(\Delta l \%)$, the reduciton of area (RA\%), yield strength $\left(\sigma_{y}\right)$ or $0.2 \%$ offset yield strength $\left(\sigma_{0.2 \% y}\right)$, ultimate tensile strength $\left(\sigma_{U T S}\right)$, and fracture strain $\left(\varepsilon_{\mathrm{f}}\right)$ can be obtained.

Percentage elongation and reduction of area, which is a measure of ductility, can be obtained as follows,

$$
\begin{aligned}
& \Delta l(\%)=\frac{\left(l_{f}-l_{0}\right)}{l_{0}} \times 100 \\
& \operatorname{RA}(\%)=\frac{\left(A_{0}-A_{f}\right)}{A_{0}} \times 100
\end{aligned}
$$

where $l_{0}$ is initial gage length, $l_{f}$ is the gage length at fracture, $A_{0}$ is the initial cross sectional area and $A_{f}$ is the cross-sectional area of the specimen 
at fracture.

The yield strength $\left(\sigma_{y}\right)$ is the stress level at which plastic deformation starts. However, austenitic stainless steel, which has a fcc crystal structure, does not show the definite yield point, while bcc structure materials exhibit a sharp yield point. Thus, an offset amount of 0.2 percent is used for the standard strength equivalent to the yield point, which is called " $0.2 \%$ offset yield strength $\left(\sigma_{0.2 \% y}\right)$ ". $0.2 \%$ offset yield strength is found by drawing a parallel line to the elastic region, having an intersection on the strain axis at a strain equal to 0.002, as illustrated in Fig. 2.7. An interception between the $0.2 \%$ offset line and the stress-strain curve represents the yield strength at $0.2 \%$ offset. The ultimate tensile strength $\left(\sigma_{U T S}\right)$ is the maximum engineering stress level. At this point, the specimen can withstand the highest stress before necking takes place.

As mentioned before, the tensile tests are carried out in hydrogen gas and in an inert environment. The ratio between hydrogen and air results represents the effect of hydrogen on mechanical properties. Relative properties are calculated as follows

Relative $0.2 \%$ offset yield strength: $\quad$ rel. $\sigma_{0.2 \% \mathrm{y}}=\frac{\sigma_{0.2 \% y}\left(\mathrm{H}_{2}\right)}{\sigma_{0.2 \% y}(\text { air })} \times 100$

Relative ultimate tensile strength: $\quad$ rel. $\sigma_{\mathrm{UTS}}=\frac{\sigma_{U T S}\left(H_{2}\right)}{\sigma_{U T S}(\text { air })} \times 100$

Relative reduction of area (RRA): $\quad \mathrm{RRA}=\frac{R A\left(\mathrm{H}_{2}\right)}{R A(\text { air })} \times 100$

Relative values close to $100 \%$ indicate full resistance $\mathrm{HE}$ and decreasing values a higher susceptibility.

Fig. 2.8 shows the parameters obtained from tensile tests plotted as a function of hydrogen content in bulk for C-0.5Mo steel grade [79]. The greater slope of the curve indicates the higher susceptibility of the material to HE. 


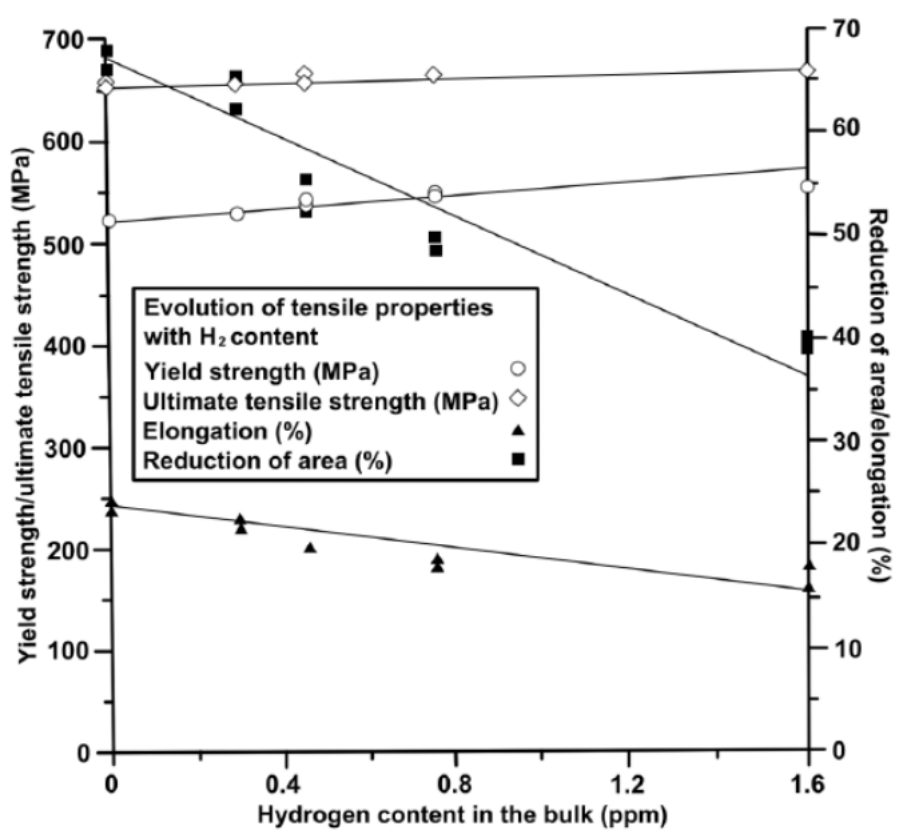

Fig. 2.8 Evolution of tensile parameters with increasing hydrogen content in the bulk for C-0.5Mo steel grade [79]. Reduction of area is a very sensitive parameter for quantifying the HE effect, while yield and tensile strengths are nearly invariant. (Figure from Pillot [79])

Reduction of area is a very sensitive parameter for quantifying the $\mathrm{HE}$ effect, while yield and tensile strengths are nearly invariant, as can be seen in Fig. 2.8. Thus, this study focuses mainly on the RRA-value. The results of relative 0.2 offset yield strength and relative ultimate tensile strength of the low־nickel steels used in this study can be found in the thesis of M. Martín $[20]$.

\subsubsection{Effect of test variables}

The embrittlement of stainless steels depends on a test variables such as hydrogen pressure, purity, strain rate, and test temperature since the degree of $\mathrm{HE}$ is governed by the rate of hydrogen reaction at metal surface. In this section these variables will be briefly discussed. Details on some extensive experiments and investigations regarding these factors are found in studies by Jewitt [80], San Marchi [81] and Michler [82]. 


\subsubsection{Hydrogen gas pressure}

Fig. 2.9 shows the effect of hydrogen pressure on the relative reduction of area (RRA) of austenitic stainless steels at room temperature [6].

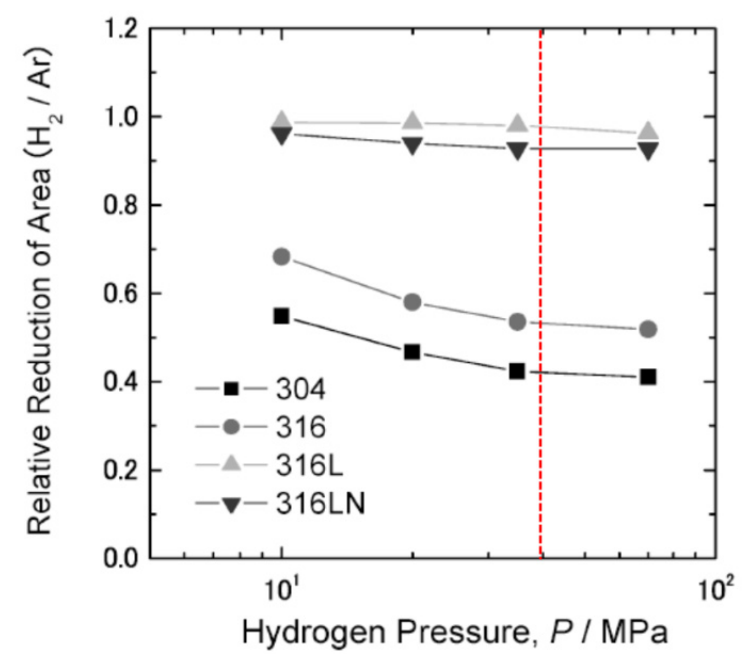

Fig. 2.9 Effect of hydrogen pressure on relative reduction in area of austenitic stainless steels at room temperature [6]. Red dotted line indicates the hydrogen pressure (40 MPa) used in this study. Reduction in area of AISI 304 and 316 decreases with increasing hydrogen gas pressure. (Figure from Fukuyama [6])

The RRA of AISI 304 and 316 decreases with increasing hydrogen pressure while Walter reported that AISI 316 stainless steel does not show a significant change in RRA [83]. Nevertheless, the ductility of unstable austenitic stainless steels such as AISI 304 is affected by the hydrogen pressure. In case of other steels such as low alloy steels and Ni based alloy (Inconel), which are more susceptible materials to $\mathrm{HE}$, the degree of embrittlement is proportional to the square root of the hydrogen gas pressures in the range of $0.1-69 \mathrm{MPa}$ [84]. This hints a clear impact of solute hydrogen in bulk on the HE behavior via Sieverts' relationship (see 2.2.4). It should be, however, stressed that HE can be significant even at much lower hydrogen gas pressure like $0.1 \mathrm{MPa}$, as shown in the next section.

\subsubsection{Hydrogen gas composition}

The purity of the gas affects the mechanical performance. The presence of impurity gas such as $\mathrm{O}_{2}, \mathrm{SO}_{2}, \mathrm{CO}$, and $\mathrm{CO}_{2}$ inhibit the entry of hydrogen 
into materials [47]. Fig. 2.10 shows the influence of oxygen on crack growth in $\mathrm{H}-11$ steel.

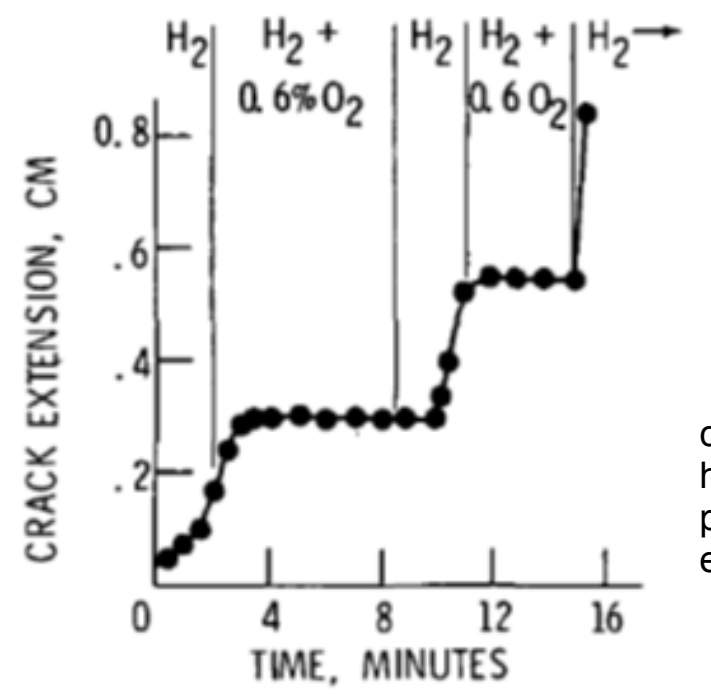

Fig. 2.10 Effect of hydrogen purity on crack growth in $\mathrm{H}-11$ steel at $23{ }^{\circ} \mathrm{C}$ and a hydrogen pressure of $0.1 \mathrm{MPa}$. The presence of oxygen inhibits the crack extension. (Figure from ASTM [47])

The crack extension can be started with the introduction of pure hydrogen, and a running crack can be inhibited by the introduction of hydrogen / oxygen gas mixtures. In this study, pure hydrogen gas $(\geq$ $99.9999 \% \mathrm{H}_{2}$ ) was used. It is clearly seen in Fig. 2.10 that the introduction of hydrogen of $0.1 \mathrm{MPa}$ immediately induced crack propagation. The oxygen content in hydrogen gas used in this study is less than $0.2 \mathrm{ppm}$ and other impurities are less than $0.5 \mathrm{ppm}$ [85]. Thus the influence of impurities can be neglected.

\subsubsection{Test temperature}

HE occurs within a specific temperature range. For austenitic stainless steel, the most severe damage occurs at around $-50{ }^{\circ} \mathrm{C}$ to $-70{ }^{\circ} \mathrm{C}$ [82]. For instance, Fig. 2.11 represents the effect of testing temperature on the relative reduction of the area of the austenitic stainless steels. 


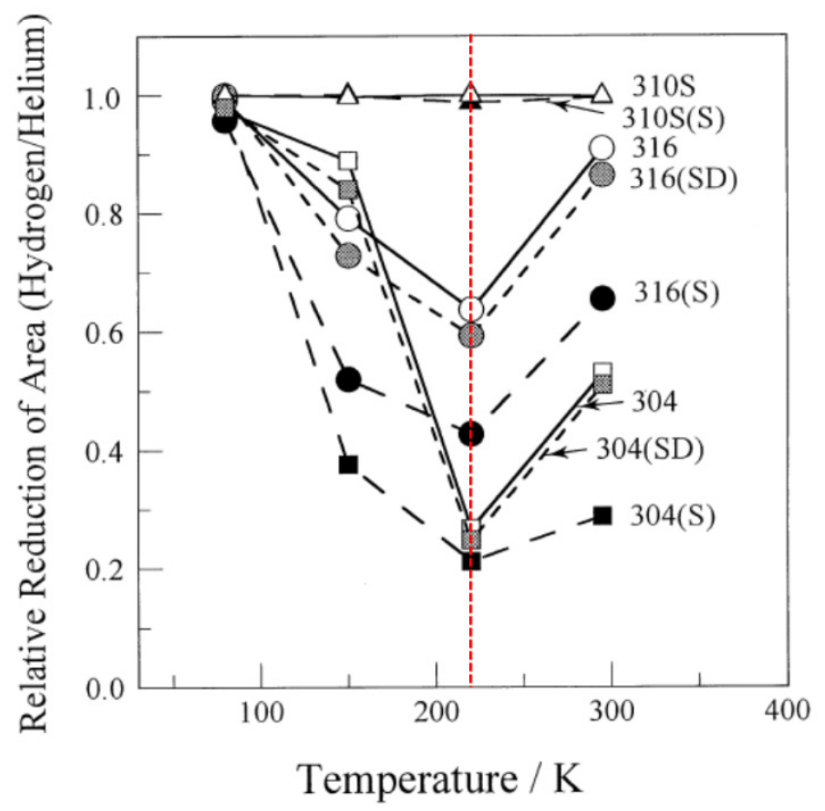

Fig. 2.11 Effect of testing temperature on the relative reduction of area of the austenitic stainless steels in hydrogen and helium of $1 \mathrm{MPa}$. (S):sensitized, (SD): desensitized, without mark: solution annealed. Red dotted line indicates the temperature $\left(-50^{\circ} \mathrm{C}\right)$ used in this study. (Figure from Michler [82])

The relative reduction of area of types 304 and 316 decreases with decreasing temperature, reaches a minimum at around $-50{ }^{\circ} \mathrm{C}$ and then increases with further temperature decrease. Based on this, $-50{ }^{\circ} \mathrm{C}$ was adapted to the test temperature in this study, to obtain the maximum effect.

\subsubsection{Strain rate}

For tensile tests in a hydrogen atmosphere, the mechanical properties have been found to be dependent on strain rate. Fig. 2.12 shows the reduction of area of several steels tested in hydrogen as a function of strain rate [82].

It can be seen that reduction of area decreases with decreasing strain rate. The strain rate dependence is a result of the rate controlling steps such as hydrogen transport and hydrogen surface reactions. At higher strain rate, there is no sufficient time for both hydrogen transport and surface reactions. Thus, tensile tests at high strain rate do not properly reflect the influence of hydrogen on materials. To evaluate the susceptibility of materials to $\mathrm{HE}$, 
slow strain rates below $10^{-3}$ to $10^{-5} \mathrm{~s}^{-1}$ are decided to be adopted [82].

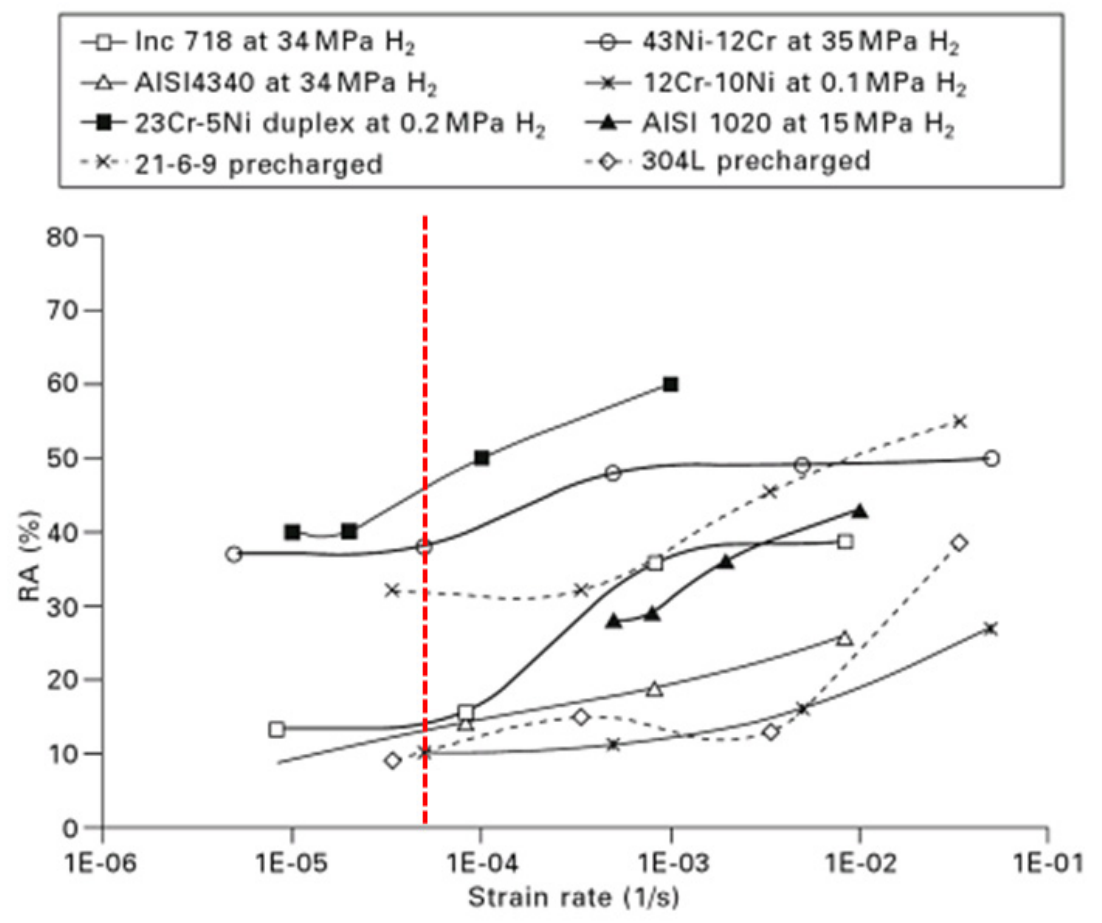

Fig. 2.12 Reduction in area of smooth cylindrical specimens tested in hydrogen atmosphere or precharged with hydrogen [82]. Red dotted line indicates the strain rate $\left(5.5 \times 10^{-5} \mathrm{~s}^{-1}\right)$ used in this study. Reduction of area decreases with decreasing strain rate resulting of the rate controlling steps. (Figure from Michler [82])

\subsubsection{Permeability, diffusivity and solubility of hydrogen}

In order to understand the HE mechanism of steels, it is important to figure out the hydrogen transport behavior in a metal. In general, hydrogen diffusivity at room temperature in bcc stainless steels is five orders of magnitude higher than that in fcc stainless steels [16]. On the contrary, hydrogen solubility in bcc stainless steel is significantly less than that of fcc stainless steels [16]. Not only the crystal structure, but also the chemical composition of the stainless steels may affect the hydrogen permeability, diffusivity and solubility. As it is already shown in Fig. 1.3, there is the tendency that RRA values increases with increasing $\mathrm{Ni}$ content. The variation of $\mathrm{Ni}$ content is significant in this study, as well. We address here the impact of alloy composition on the hydrogen permeability, on the 
diffusivity and the solubility, by making a comparison of literature data on a variety of steels.

For the investigation of the hydrogen transport behavior, hydrogen permeation method is employed either gas phase or in electrochemical environment (cathodic charging). Many researchers have reported on the permeability of hydrogen in several austenitic stainless steels measured in a wide range of temperature $\left(100-927^{\circ} \mathrm{C}\right)$ by hydrogen gas permeation technique while cathodic charging is only able to obtain data near room temperature. This section summarizes on previously reported values of hydrogen permeability, diffusivity and solubility in austenitic stainless steels.

In the following, the principle of gas permeation will be described by reference to [86-88]. Gas permeation is defined as a transfer of gas from a high pressure side to a low pressure side across a membrane. The permeation process is most commonly modeled by Fick's first law which can be written as

$$
\mathrm{J}=-\mathrm{D}\left(\frac{d c}{d x}\right)
$$

where $\mathrm{J}$ is the flux of hydrogen atoms through the membrane, $\mathrm{D}$ is the diffusion coefficient of the membrane, and $c$ is the concentration of hydrogen atoms.

For the steady state in a semi-infinite plane, the concentration gradient can be postulated as a constant and independent of $x$. Then, Eq. (2-18) can be modified to a simple formula as follows,

$$
\mathrm{J}_{\infty}=\mathrm{D}\left(\frac{C_{1}-C_{2}}{d}\right)
$$

where $\mathrm{J}_{\infty}$ is the steady state diffusional flux, $C_{1}$ and $C_{2}$ are the hydrogen concentrations at the high pressure side and the low pressure side, respectively, and $\mathrm{d}$ is the thickness of the membrane.

When the hydrogen concentration follows the Sieverts' law, the hydrogen 
concentration at the respective surfaces can be described as follows.

$$
\begin{aligned}
& C_{1}=\mathrm{K} \sqrt{P_{1}} \\
& C_{2}=\mathrm{K} \sqrt{P_{2}}
\end{aligned}
$$

where $P_{1}$ and $P_{2}$ are the pressures on the respective sides of the membrane, and $\mathrm{K}$ is the solubility coefficient. Thus, Eq. (2-19) can be written as,

$$
\mathrm{J}_{\infty}=\frac{D K}{d}\left(\sqrt{P_{1}}-\sqrt{P_{2}}\right)
$$

The hydrogen pressure at the low pressure side, which is vacuum pressure, is negligible. Then, the diffusional flux can be expressed as

$$
\mathrm{J}_{\infty}=\frac{D K}{d} \sqrt{P}
$$

Since the product $D K$ is defined as the permeability $\Phi$, Eq. (2-23) yields,

$$
\Phi=\mathrm{DK}=\frac{\mathrm{J}_{\infty} d}{\sqrt{P}}
$$

Both permeability and diffusivility are strongly dependent on temperature. D and $\Phi$ may be expressed in terms of the temperature in Arrhenius form,

$$
\begin{aligned}
& \Phi=\Phi_{0} \exp \left(\frac{-H_{\Phi}}{R T}\right) \\
& \mathrm{D}=\mathrm{D}_{0} \exp \left(\frac{-H_{D}}{R T}\right)
\end{aligned}
$$

where $\Phi_{0}$ and $\mathrm{D}_{0}$ are the permeability and the diffusion constant, $H_{\Phi}$ and $H_{D}$ are the enthalpies of permeation and diffusion, respectively.

Since $\Phi=$ DK, the solubility $\mathrm{K}$ is given by the ratio of permeability and diffusivity as, 


$$
\mathrm{K}=\frac{\Phi_{0}}{\mathrm{D}_{0}} \exp \left[\frac{-\left(H_{\Phi}-H_{D}\right)}{R T}\right]
$$

According to Eq. (2-25), (2-26) and (2-27), permeability, diffusivity and solubility can be plotted as a function of reciprocal temperature, known as Arrhenius plot.

Summarized data points are plotted in Fig. 2.13 (permeability), Fig. 2.14 (diffusivity) and Fig. 2.15 (solubility) as Arrhenius type plots. In general, the permeation measurements are conducted in the wide temperature range. As a result, the reported results consist of many data points. In order to simplify the figures, only two selected points, which are at high temperature and at the lowest temperature, are plotted in Fig. 2.13, Fig. 2.14 and Fig. 2.15.

The criteria of the literature data section are chosen as follows. Firstly, AISI 304 and AISI 316 series were selected, since the chemical composition of these steels is almost identical to that of the reference steels in this study. To elucidate the impact of nickel on the permeability, on the diffusivity and on the solubility, $21 \mathrm{Cr}-6 \mathrm{Ni}-9 \mathrm{Mn}, 22 \mathrm{Cr}-13 \mathrm{Ni}-5 \mathrm{Mn}$, and Inconel 600 (Ni-base alloy) were selected. The chemical composition of these alloys is shown in Table 2.1. [89]

Table 2.1 Chemical composition of different important steel types [89]

\begin{tabular}{|c|c|c|c|c|c|c|c|}
\hline & $\mathrm{C}$ & $\mathrm{Mn}$ & $\mathrm{Cr}$ & $\mathrm{Ni}$ & $\mathrm{Mo}$ & $\mathrm{N}$ & $\mathrm{Fe}$ \\
\hline 304 & 0.08 & 2 & $18.0-20.0$ & $8.0-11.0$ & - & - & bal. \\
\hline 316 & 0.08 & 2 & $16.0-18.0$ & $10.0-14.0$ & $2.00-3.00$ & - & bal. \\
\hline $21-6-9$ & 0.08 & $8.00-10.00$ & $19.0-21.50$ & $5.5-7.5$ & - & $0.15-0.40$ & bal. \\
\hline $22-13-5$ & 0.03 & $4.00-6.00$ & $20.5-23.5$ & $11.5-13.5$ & $2.00-3.00$ & $0.20-0.40$ & bal. \\
\hline Inconel 600 & 0.15 & 1 & $14.0-17.0$ & 72 & - & - & $6.0-10.0$ \\
\hline
\end{tabular}

The composition of AISI 304 and AISI 316 is nearly equivalent to the samples W11 and W12 of this study, respectively. These alloys are used as reference steels in this study. The alloy of 21-6-9 has less Ni content compared to AISI 304 and AISI 316. The alloy of Inconel 600 contains more than $70 \mathrm{wt} \%$ of Ni. Thus, these two, $21 \mathrm{Cr}-6 \mathrm{Ni}-9 \mathrm{Mn}$ and Inconel 600 , are selected to evaluate the impact of the composition difference in the results in this study.

Further, the surface condition also strongly influences on the 
measurement quantities in permeation studies since oxide layers act as a diffusion barrier [88]. To cancel out this surface effect on the permeability data in our comparison, the reference data used here are mainly collected exclusively from those results with surface $\mathrm{Pd}$ platings or $\mathrm{Pd}$ sputter coatings and so on. For these samples, surface oxides can be neglected.

Fig. 2.13 shows the hydrogen permeability through austenitic stainless steels. For comparison, permeability data for $\alpha-\mathrm{Fe}, \mathrm{Ni}$ and $\mathrm{Pd}$ are also plotted in this figure.

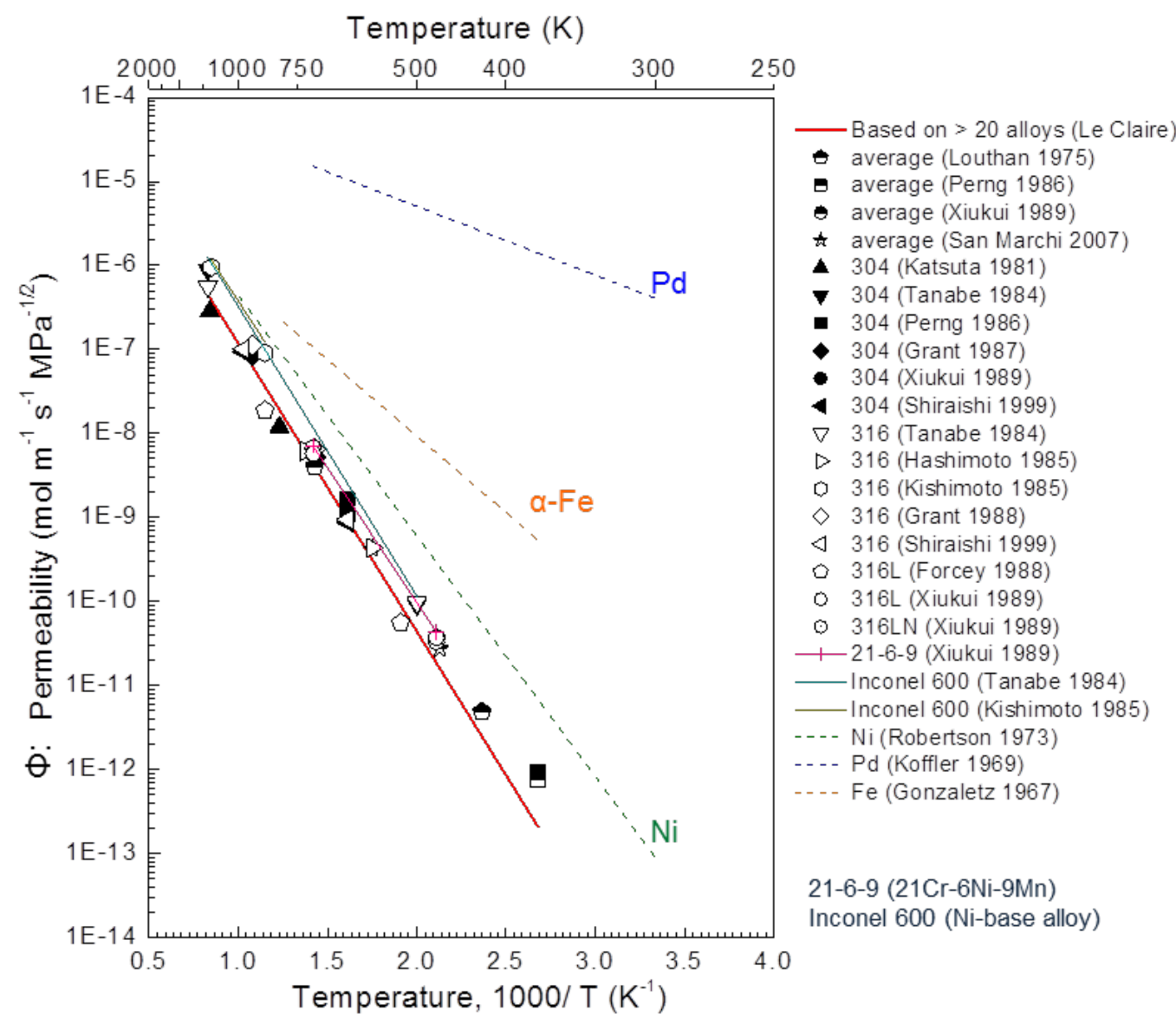

Fig. 2.13 Permeability of hydrogen in austenitic stainless steels, $\mathrm{Pd}, \alpha-\mathrm{Fe}$ ad Ni. Permeability values reported by several researchers are in reasonable agreement with each other. The permeability data for several austenitic stainless steels and reference number is listed in Appendix (Table 8.1 and Table 8.2).

LeClaire reported that most data of hydrogen permeability in austenitic stainless steels is within factors of 1.5 and $1 / 1.5$ of the average curve [90], suggesting that a fair agreement has been observed among different studies. In fact, permeability values reported by several researchers are in 
reasonable agreement with each other as shown in Fig. 2.13. Interestingly, hydrogen permeability among these different austenitic stainless steels are possibly less sensitive to the alloy composition. Activation parameters of $\mathrm{H}$ permeation used in Fig. 2.13 are listed in Appendix.

Fig. 2.14 summarises the hydrogen diffusivity in austenitic stainless steels. For comparison, the $\mathrm{H}$-diffusivity in $\mathrm{a}-\mathrm{Fe}$, in $\mathrm{Ni}$ and in $\mathrm{Pd}$ are also plotted in Fig. 2.14.

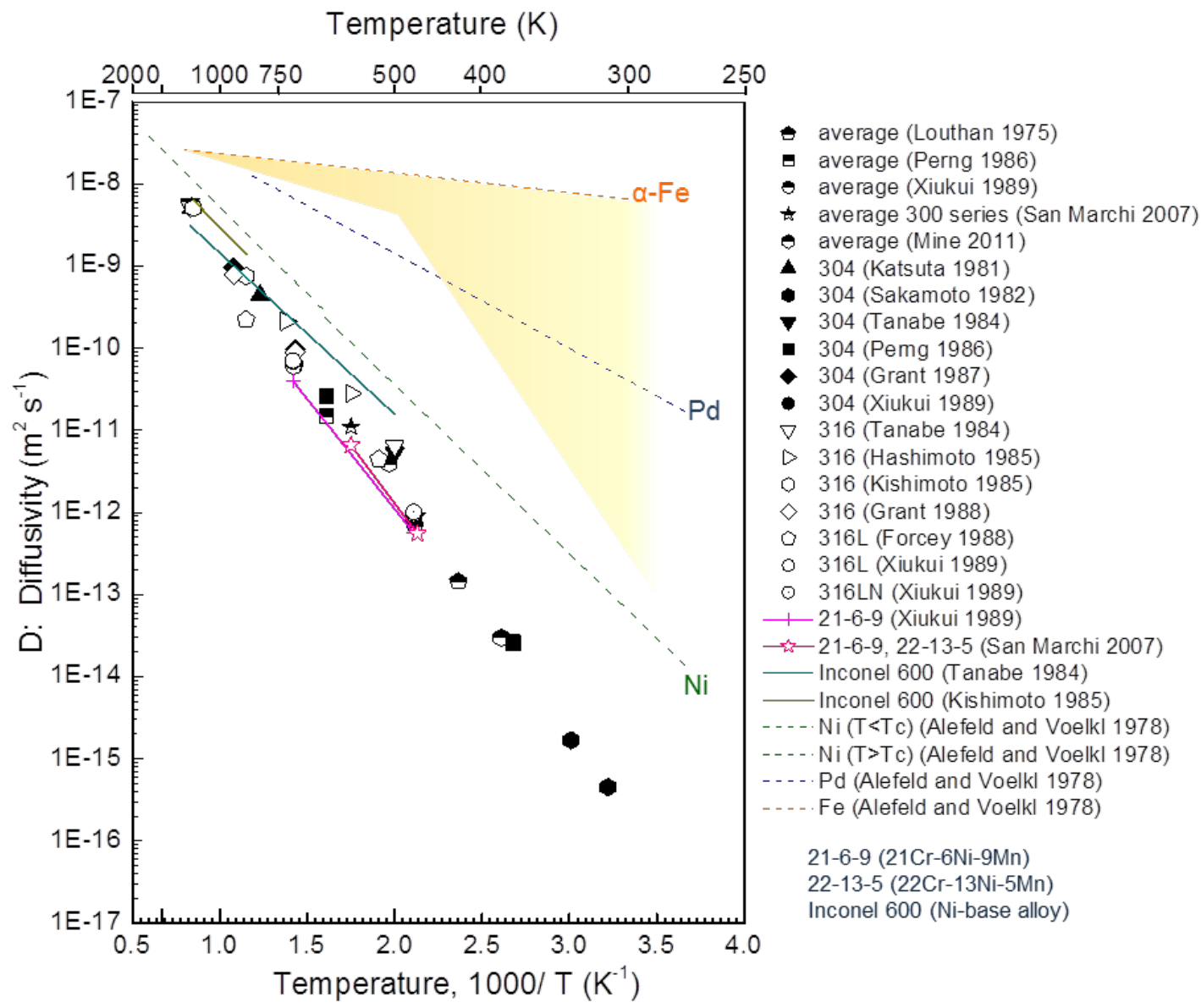

Fig. 2.14 Diffusivity of hydrogen in austenitic stainless steels, Pd, $\alpha$-Fe ad Ni. The permeability data for several austenitic stainless steels and reference number is listed in Appendix (Table 8.1 and Table 8.2)

The results of diffusion measurement for $\alpha-F e$ often show quite unsatisfactory scatter especially at temperatures below $250{ }^{\circ} \mathrm{C}(523 \mathrm{~K})$ [91]. According to Alefeld and Völkl, this large scatter may be due to the difference of surface the sample's conditions [91]. This scatter region is indicated in 
light orange shade in Fig. 2.14.

Nevertheless, the hydrogen diffusivities in austenitic stainless steels are somewhat less consistent among the four types of alloys compared to the permeability data, shown in Fig. 2.13. This, therefore, implies an impact of the chemical composition on the hydrogen diffusivity in austenitic stainless steels. As one can see, that the diffusion relationship of Inconel 600 (Ni based alloy), marked in green, appears close to that of $\mathrm{Ni}$ and the relationship of high chromium content alloys (see red lines) are scaled somewhat lower than that of AISI 304 and 316 (bold symbols). Some researchers have mentioned that the hydrogen diffusivity in austenitic stainless steels tends to scale with the inverse of the chromium content [88,92]. Tanabe et al. examined the contribution of chromium to the hydrogen diffusivity in 9 types of austenitic stainless steels with $15-25 \mathrm{wt} \%$ of chromium. They roughly estimated the activation energy of the hydrogen diffusivity for fcc chromium (hypothetical phase). Tanabe et al. interpreted that this inverse correlation in diffusivity is due to the high activation energy $(60 \mathrm{~kJ} / \mathrm{mol})$ of the $\mathrm{H}$-diffusion in fcc chromium compared with that in fcc Fe $(44.9 \mathrm{~kJ} / \mathrm{mol})$ and $\mathrm{Ni}(40 \mathrm{~kJ} / \mathrm{mol})$.

The hydrogen diffusivity in AISI 304 and 306 shows a good agreement among different studies, especially at temperature below 500 K. In Fig. 2.14, diffusion data obtained at around room temperature by electrochemical method, is also plotted. A consistency with the high temperature data is observed here. According to this, it is not unreasonable to extrapolate the hydrogen diffusivity in AISI304 and 306 to ambient temperature.

Fig. 2.15 shows the hydrogen solubility in austenitic stainless steels. Obviously the large scatter becomes significant here, because this hydrogen solubility is obtained by the ratio of permeability and diffusivity data as described in (2-27). According to the study by Martín, the hydrogen concentration of austenitic stainless steels is 45 wtppm at $25{ }^{\circ} \mathrm{C}, 40 \mathrm{MPa}$ hydrogen pressure, while that of ferritic stainless steels with bcc crystal structure is 0.32 wtppm [16]. 


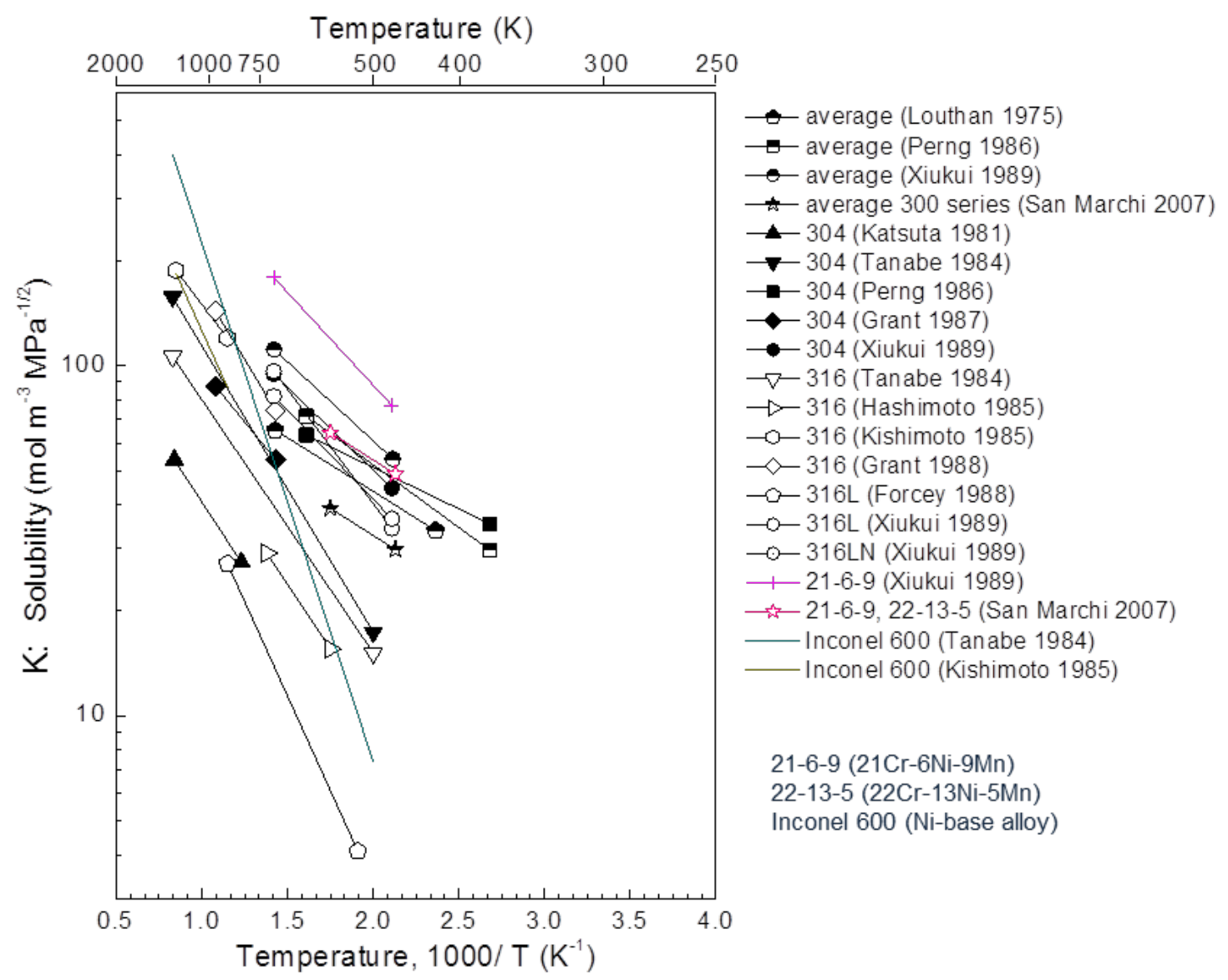

Fig. 2.15 Solubility of hydrogen in austenitic stainless steels. The permeability data for several austenitic stainless steels and reference number is listed in Appendix (Table 8.2)

\subsection{Oxide layer formation}

The high temperature oxidation is accompanied with heat treatments of steels. The heat treatments are often necessary for stainless steels, in order to achieve desired material properties. Solution annealing is one of the heat treatment processes. The purpose of this treatment is to restore the material to its original condition, removing alloy segregation, sensitization, and restoring ductility after cold working.

In this study, the austenitic stainless steels are solution annealed around $1050{ }^{\circ} \mathrm{C}$, depending on the chemical composition of austenitic stainless steels, followed by quenching in argon gas. During solution annealing, oxide layer formed on stainless steels and its thickness and composition could strongly be affected by the annealing conditions such as a gaseous environment in a 
furnace, annealing duration, etc. The difference in the chemical components of the stainless steel also changes a composition of the oxide layer.

In this chapter, the principal of the oxide formation at high temperature is described toward a better understanding of the oxide layers formed on the studied samples.

\subsubsection{Oxidation condition - Ellingham diagram}

Some components in steels react strongly with oxygen during the annealing process. For determination of whether or not a given component in an alloy reacts with oxygen or other gases under applied conditions, the second law of thermodynamics is an important tool. The reaction equilibrium between a solid metal $\mathrm{M}$, its oxide $\mathrm{MO}_{2}$, and oxygen gas at the temperature $T$ and the pressure $P$ can be written as

$$
M+O_{2}=M O_{2}
$$

if the activities of $M$ and $\mathrm{O}_{2}$ are taken as unity, the standard free energy of formation $\Delta G^{\circ}$ can be written as

$$
\Delta G^{\circ}=-R T \ln K_{R}=R T \ln p_{O_{2}}
$$

where $K_{R}$ is equilibrium constant, $p_{\mathrm{O}_{2}}$ is partial pressure of oxygen. Then, the dissociation pressure can be written as

$$
p_{O_{2}}=1 / K_{R}
$$

Fig. 2.16 shows Ellingham diagrams plotted the experimentally determined variation of $\Delta G^{\circ}$ with $T$ for the oxidation of a series of metals [93]. The elements related this study are highlighted in colors. The oxidation reaction of Mo and $\mathrm{Fe}$ are calculated from the thermodynamics data and added to the original graph. A vertical red dashed line indicates the solution annealing temperature used in this study. The values of $\Delta G^{\circ}$ are expressed as $\mathrm{kJ} / \mathrm{mol}$. 


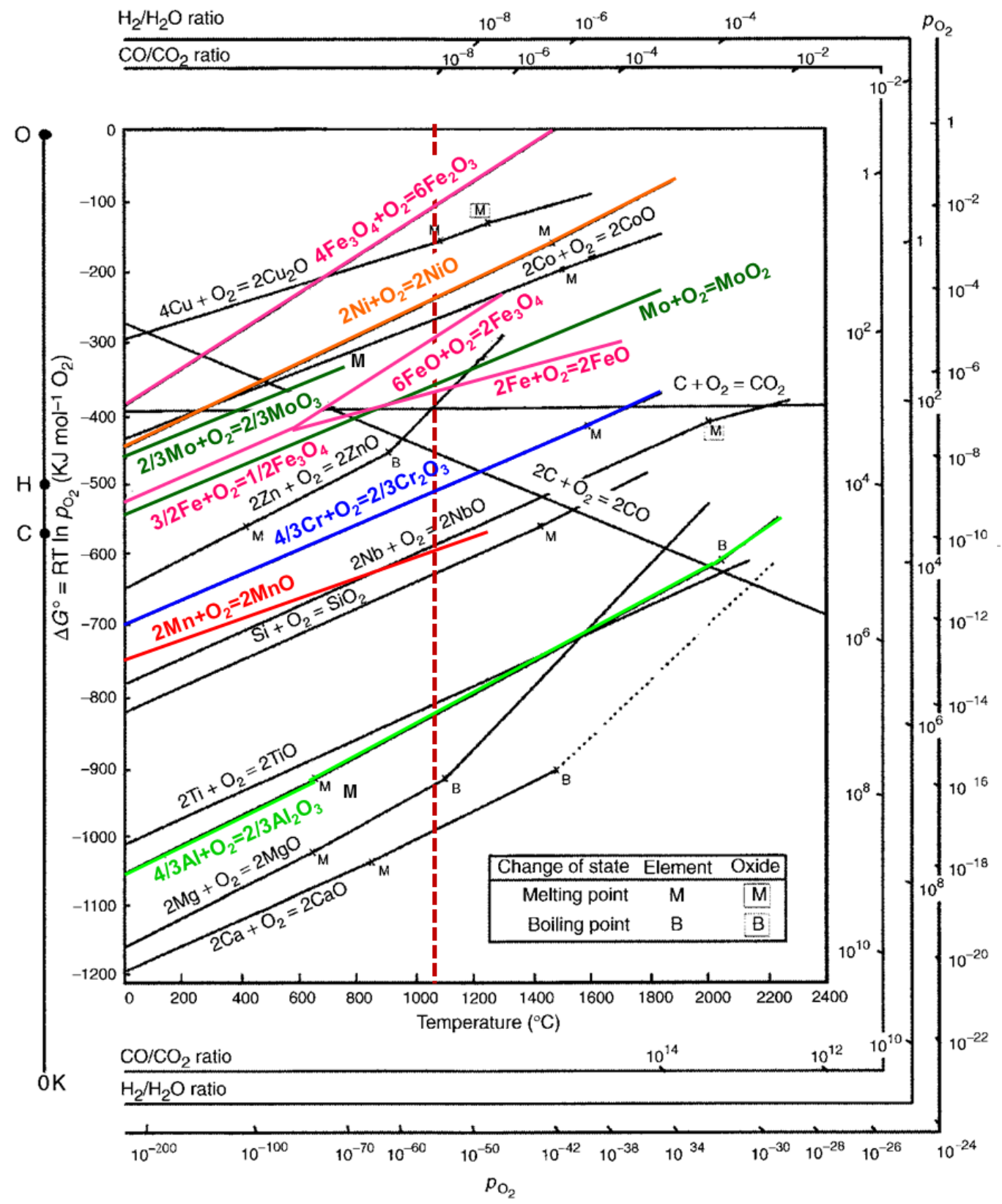

Fig. 2.16 Standard free energy of formation of selected oxides as a function of temperature [93,94]. Vertical dotted line indicates solution annealing temperature applied in this study. The oxidation reactions of Mo and Fe are added from the themodynamics data. The lower the position of the line on the diagram the more stable is the oxide. (Figure from Birks [93])

The lower the position of the line on the diagram the more stable is the oxide. The nomographic scales of the partial pressure $p_{O_{2}}$, the $\mathrm{H}_{2} / \mathrm{H}_{2} \mathrm{O}$ ratio and the $\mathrm{CO} / \mathrm{CO}_{2}$ ratio are plotted to the Ellingham diagram along the right-edge and along the bottom edge. The dissociation pressure can be obtained by drawing a straight line from the origin (marked $\mathrm{O}$ ) through the 
free energy line at the temperature of interest and reading the oxygen partial pressure from the intersection with the scale on the right side (labeled $p_{\mathrm{O}_{2}}$ ).

For multi-component systems like steels, different oxides are expected to appear at the sample surface and in the samples. In this regard, we can predict the plausible oxide from the Ellingham diagram. For example, when a stainless steel containing aluminum is solution annealed at $1050{ }^{\circ} \mathrm{C}, \mathrm{Al}_{2} \mathrm{O}_{3}$ forms as an oxide layer. This is due to the fact that $\mathrm{Al}_{2} \mathrm{O}_{3}$ is more stable than the oxides of the other components, resulting in the preferential oxidation of aluminum.

\subsubsection{Mechanism of oxidation}

For oxide layer growth, metal ions, oxygen ions and electrons must be transported through the oxide layer. Since all metal oxides are ionic in nature, it is not appropriate to consider the transport of neutral metal atoms and non-metal atoms. Thus, it is necessary to postulate that ions or electrons migrate through the oxide layer. Fig. 2.17 gives ionic and electronic transport process according to Wagner's theory for the oxide layer formation and growth [93].

The partial steps of the oxidation are as follows,

(1) Diffusion of metal species in the solid

(2) Oxidation reaction of metal at the metal / oxide interface

(3) Migration of ions or electrons across the oxide

(4) Reduction reaction of oxygen at the oxide / gas interface

This general oxidation process becomes more complex when different constituents have to be considered as it is the case for steels. 


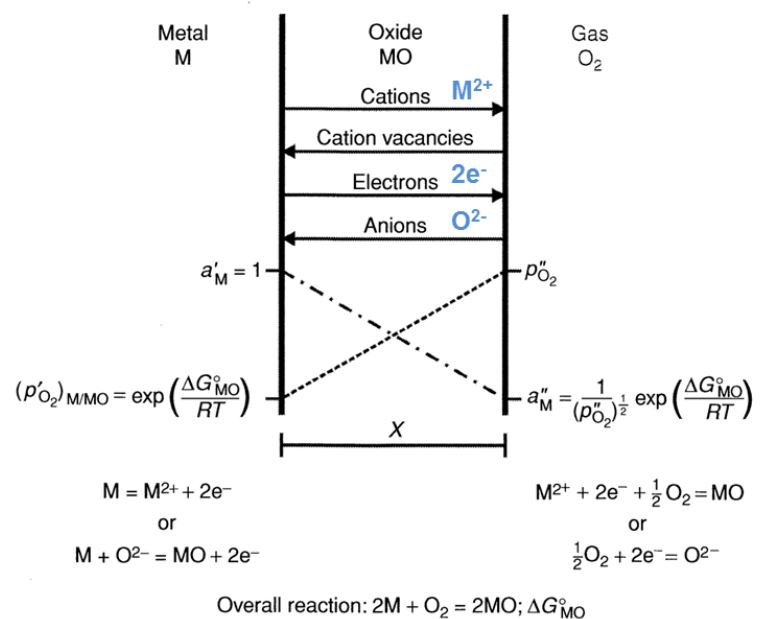

Fig. 2.17 Diagram of scale formation according to Wagner's model. (Figure from Birks [93])

\subsubsection{Transport mechanism}

Crystalline oxides which form as the result of oxidation of a metal are generally non-stoichiometric and thus the metal to non-metal atom ratio is not exactly given by a chemical formula, even though the compound is electrically neutral. Non-stoichiometric compounds are classified as semiconductors and may show positive (p-type) or negative ( $n$-type) behavior. The possible oxides formed in stainless steel are listed separately for the $\mathrm{p}^{-}$ or n-type in Table 2.2 [94].

Table 2.2 Electrical conductivity of oxides [94]

$$
\begin{aligned}
& \text { p-type (Metal-deficit semiconductors) } \\
& \mathrm{Cr}_{2} \mathrm{O}_{3}, \mathrm{FeCr}_{2} \mathrm{O}_{4}, \mathrm{MnO}, \mathrm{Mn}_{3} \mathrm{O}_{4}, \mathrm{Mn}_{2} \mathrm{O}_{3}, \mathrm{FeO}, \mathrm{NiO} \\
& \text { n-type (Metal-excess semiconductors) } \\
& \mathrm{Fe}_{2} \mathrm{O}_{3}, \mathrm{MoO}_{3}, \mathrm{Al}_{2} \mathrm{O}_{3}
\end{aligned}
$$

The p-type metal-deficit oxides contain metal cation vacancies. Cation can diffuse by cation vacancies, to the oxide/gas interface where they react with adsorbed oxygen. Electrons migrate by electron holes, to the adsorbed oxygen atoms, which then become oxygen anions. While cations and electrons move outward through the scale toward the gas, cation vacancies 
and electron holes move inward toward the metal. Consequently, as the scale thickness, the cation vacancies tend to accumulate to form voids at the metal/oxide interface [95]

The n-type metal-excess semiconductor oxides is anion deficient. The oxide grows at the metal/oxide interface by inward diffusion of $\mathrm{O}^{2-}$ through the anion vacancies in the oxide [95].

A multiple oxide is often formed on metals. In this case, in general, the outermost oxide is often $n$-type because of its anion vacancies, while inner oxide is a p-type because of the ease with which electron holes and cation vacancies can form[95]. The detail of $\mathrm{p}^{-}$and $\mathrm{n}$-type semiconducting oxide can be found in Ref. [93-95]. This is visible in a stacking sequence of oxides $[93,94]$.

This kind of stacking sequences is even more complicated when multicomponent materials are under consideration. A series of different oxides is expected to form at these sample surfaces. The order of the related oxide layers can, also, depend on the ion-mobility.

\subsubsection{Kinetics of oxidation}

\subsubsection{Oxidation reaction rate}

For the oxidation kinetics of metal, the following five equations are proposed.

$$
\begin{array}{ll}
\mathrm{x}=k_{l} t & (\text { Linear rate law) } \\
\mathrm{x}=k_{\log } \log \left(t+t_{0}\right)+A & (\text { Logarithmic rate law) } \\
1 / \mathrm{x}=B-k_{i l} \log t & (\text { Inverse logarithmic rate law) } \\
\mathrm{x}=\sqrt{k_{p} t} & \text { (Parabolic rate law) } \\
\mathrm{x}=\sqrt[3]{k_{c} t} & \text { (Cubic rate law) }
\end{array}
$$

where $\mathrm{x}$ is the oxide layer thickness and $\mathrm{A}, \mathrm{B}, k_{l}, k_{\log }, k_{i l}, k_{p}$ and $k_{c}$ are constants at constant temperature. These equations are schematically 


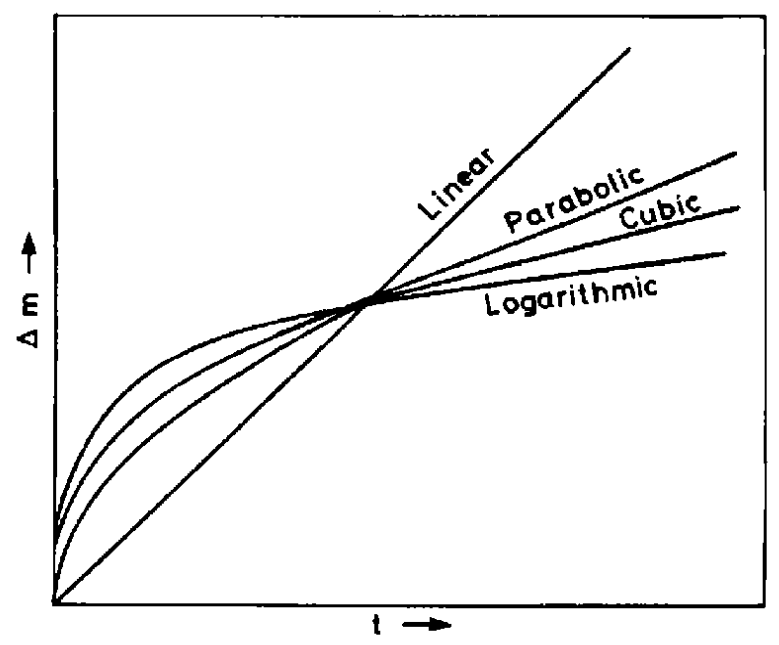

Fig. 2.18 Oxidation kinetics (Figure from Cahn[94])

represented in Fig. 2.18

The linear rate law is usually observed at the initial stages of oxidation where the growth process is controlled by surface diffusion and adsoption. The logarithmic and the inverse logarithmic rate law is characteristic for the oxidation of many metals at low temperature and for the formation of very thin oxide films below tens of nm thickness. The rate controlling step for the logarithmic law is either anion movement or electron tunneling process which creates an electric field across the oxide layer initially. The inverse logarithmic law can result when cation transport is the rate controlling step. The parabolic and the cubic rate law are observed at high temperature oxidation where the transport of ions across the oxide layer becomes the rate controlling step. The parabolic rate law follows for $\mathrm{p}$-type semiconductors and the cubic rate law for n-type semiconductors [94].

For the stainless steels, a logarithmic or inverse logarithmic rate is reported [96]. It is also expected for the steels of this study, because of the high temperature oxidation occuring during the solution annealing.

\subsubsection{Diffusion}

During oxidation, metal and oxygen ions migrate through the lattice, grain boundary, material interfaces and dislocation core. Fig. 2.19 shows 
various cations diffusivties in a $\mathrm{NiO}$ lattice, at grain boundaries and at dislocations [97].

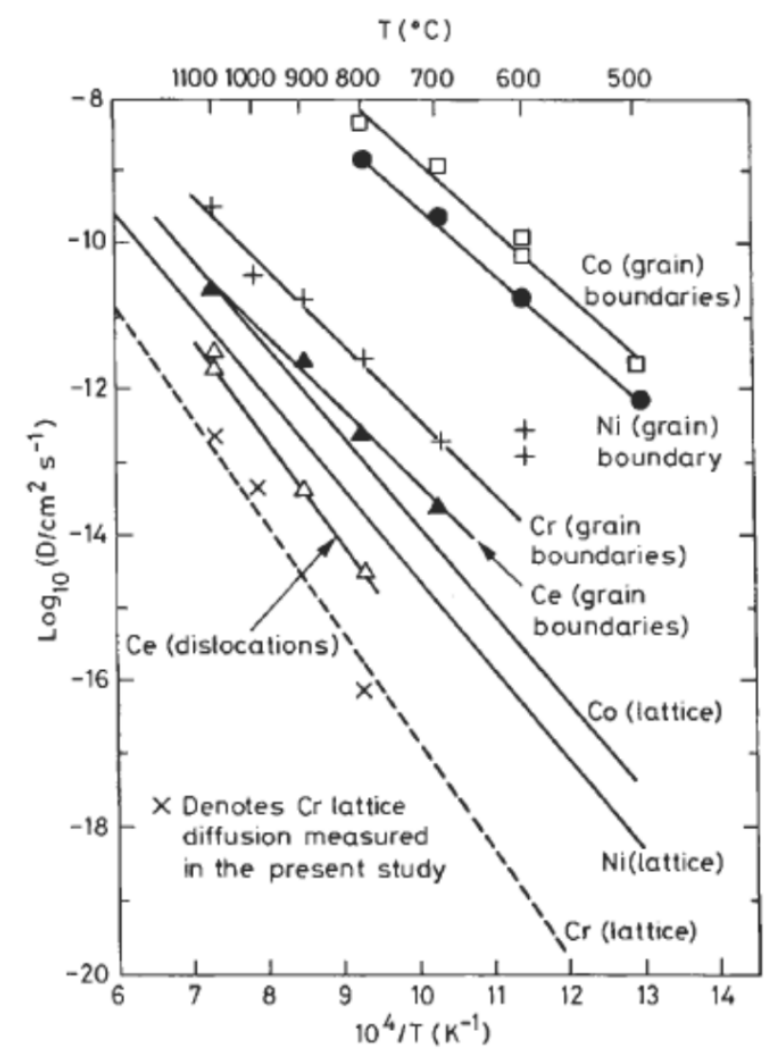

Fig. 2.19. Arrhenius plot summarising the diffusion coefficients for various impurity cations in the $\mathrm{NiO}$ lattice, grain-boundaries and dislocations (Figure from Atkinson [97])

It is found that the diffusion rates of respective cations along the grain boundaries is four to six orders of magnitude higher than in the lattice. However, in the case of the samples regarded in this work, the contribution of grain boundary diffusion to overall diffusion is expected to be relatively small, since the volume of grain boundary is extremely small compared to that of the lattice, for $50 \mu \mathrm{m}$ size grains and larger. 


\section{Experimental}

\subsection{Alloy Development and evaluation of mechanical properties}

As shown in Fig. 1.4, this work was performed in collaboration with Bochum University and seven industrial partners. The development of lean alloyed austenitic stainless steels, preparation of tensile test specimens including heat treatment process, tensile tests in air, and evaluation of mechanical properties were conducted by Bochum University. Tensile tests in hydrogen atmosphere were carried out by TWI Ltd. More detailed concept of alloy development and experimental procedures are found in Ref. [20].

\subsubsection{Chemical composition}

The chemical compositions of the investigated alloys W11-W21 are presented in Table 1. The reference steels W11 and W12 were provided by Deutsche Edelstahlwerke (DEW, Germany) as a lower and upper limit of material properties. The chemical composition of W11 and W12 is quite similar to AISI 304L and 316L, respectively.

Table 3.1 Chemical composition of the low-nikel steels, value in wt\% [16]. Fe is balanced. W11 and W12 is comparable to AISI $304 \mathrm{~L}$ and $316 \mathrm{~L}$, respectively.

\begin{tabular}{cccccccccccccc}
\hline $\begin{array}{c}\text { Internal } \\
\text { No. }\end{array}$ & $\mathrm{C}$ & $\mathrm{Si}$ & $\mathrm{Mn}$ & $\mathrm{P}$ & $\mathrm{S}$ & $\mathrm{Cr}$ & $\mathrm{Ni}$ & $\mathrm{Mo}$ & $\mathrm{Al}$ & $\mathrm{Nb}$ & $\mathrm{V}$ & $\mathrm{N}$ & $\mathrm{B}$ \\
\hline W11 & 0.018 & 0.68 & 1.96 & 0.030 & 0.030 & 17.70 & 8.49 & 0.30 & - & - & - & 0.069 & - \\
W12 & 0.012 & 0.83 & 1.43 & 0.030 & 0.020 & 17.10 & 12.20 & 2.46 & - & - & - & 0.057 & - \\
W14 & 0.025 & 0.02 & 0.06 & 0.007 & 0.006 & 14.74 & 10.62 & 0.02 & 1.640 & 0.010 & 0.010 & 0.021 & - \\
W15 & 0.013 & 0.04 & 0.06 & 0.005 & 0.004 & 14.62 & 10.83 & 0.01 & 0.001 & 0.009 & 0.011 & 0.146 & - \\
W16 & 0.172 & 0.05 & 5.90 & 0.006 & 0.008 & 17.80 & 7.89 & 0.02 & 0.001 & 0.003 & 0.021 & 0.026 & - \\
W17 & 0.022 & 0.03 & 6.04 & 0.007 & 0.006 & 17.28 & 8.84 & 0.02 & 0.001 & 0.003 & 0.011 & 0.022 & $<0.03$ \\
W18 & 0.099 & 0.05 & 0.16 & 0.006 & 0.006 & 14.67 & 10.96 & 0.02 & 0.009 & 0.062 & 0.495 & 0.014 & - \\
W19 & 0.116 & 0.05 & 10.27 & 0.010 & 0.005 & 12.98 & 7.93 & 0.03 & 2.870 & 0.008 & 0.020 & 0.020 & - \\
W20 & 0.187 & 0.20 & 5.57 & 0.012 & 0.016 & 17.91 & 8.33 & 2.59 & 0.001 & 0.008 & 0.031 & 0.059 & - \\
W21 & 0.147 & 1.95 & 9.18 & 0.008 & 0.006 & 14.33 & 8.43 & 0.02 & 0.001 & 0.005 & 0.023 & 0.035 & - \\
\hline
\end{tabular}

The other alloys were produced by Bochum University on Lab-base in order to develop lean alloyed austenitic stainless steels with high resistance to HE. To control the mictrostructure and properties of these steels, the basic 
alloy was modified by adding or subtracting a number of alloying elements. It can be confirmed that all low-nickel steels are within the austenitic region as shown in the Schaeffler diagram (Fig. 3.1).

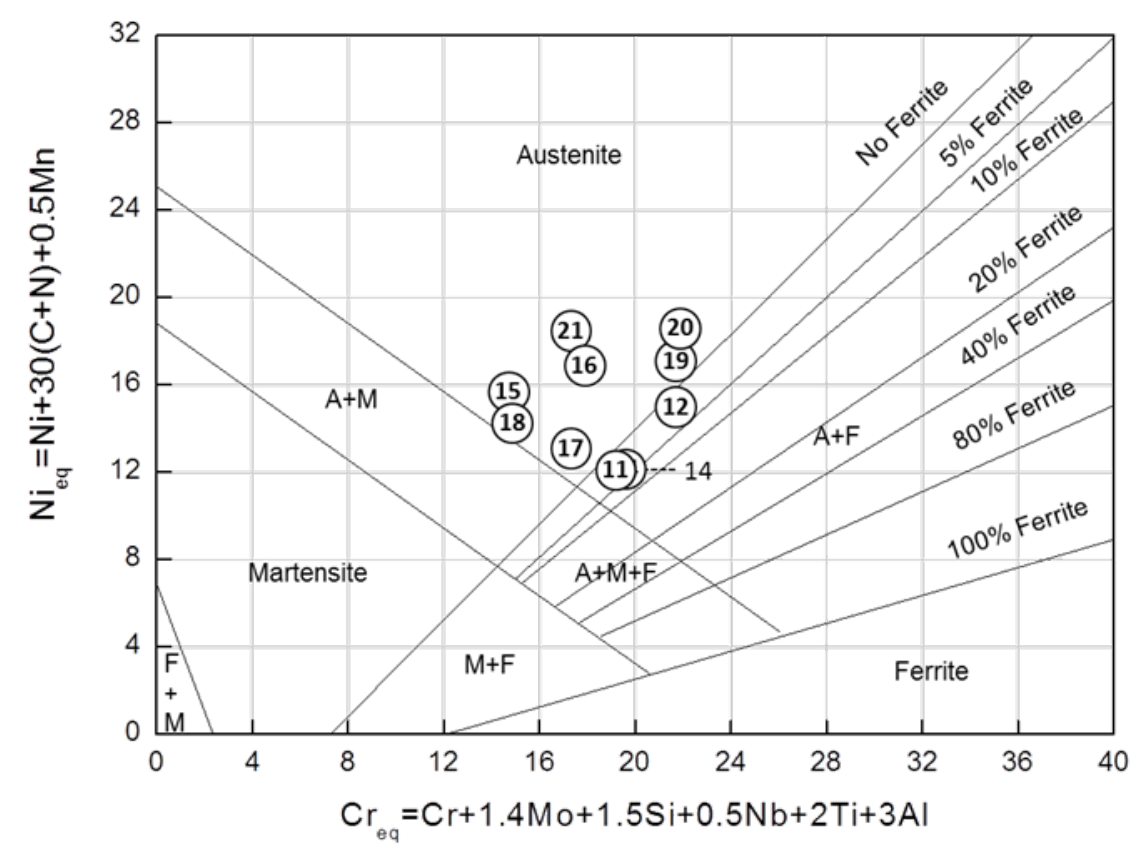

Fig. 3.1 Schaeffler diagram for the low-nickel steels. The numbers refer to the sample list in Table 3.1.

\subsubsection{Production of tensile test specimens}

To reduce the influence of alloying segregation, only one tensile specimen was machined from the centre of the bar parallel to the rolling direction. The alloys were machined into cylindrical tensile specimens with a gauge length of $30 \mathrm{~mm}$ and a diameter of $5 \mathrm{~mm}$ by wet turning. The turning process is schematically illustrated in Fig. 3.2 [16].

Due to the machining process, there is a risk of strain-induced a'-martensite formation on the sample surface [16]. Thus, in order to remove the strain-induced a'-martensite, all specimens were subsequently solution annealed in vacuum for $30 \mathrm{~min}$ at $1050{ }^{\circ} \mathrm{C}$ or at $1150{ }^{\circ} \mathrm{C}$ depending on the alloy composition. The solution annealing process leads to an a'-martensite-free surface [16]. To determine the influence of annealing time, 
alloy W11 was additionally solution annealed for $15 \mathrm{~min}$. All specimens were quenched in Ar gas at a pressure of $200 \mathrm{kPa}$.

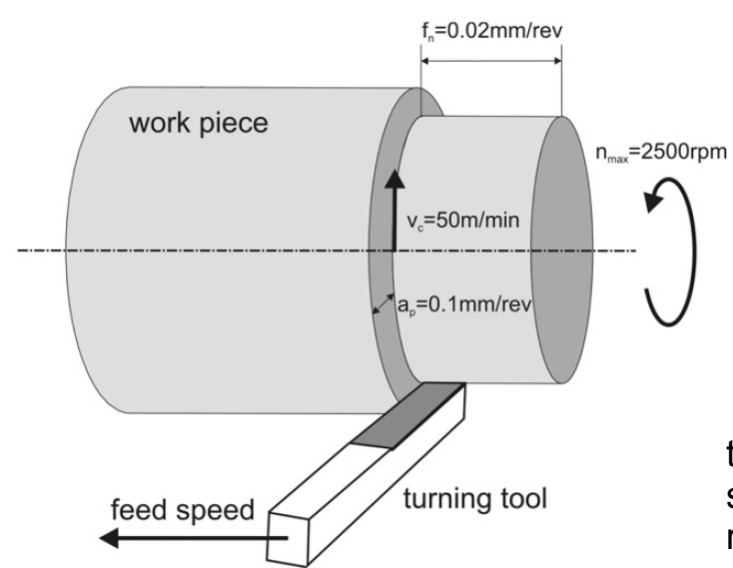

Fig. 3.2 Schematic illustration of the turning process for machining tensile samples with a defined surface roughness. (Figure from Martín [16], with permission of the author)

\subsubsection{Tensile tests}

Tensile tests were performed at $25{ }^{\circ} \mathrm{C}$ and at $-50{ }^{\circ} \mathrm{C}$ in a high purity hydrogen gas $\left(\geq 99.9999 \% \mathrm{H}_{2}\right)$ at $40 \mathrm{MPa}$ and in air at atmospheric pressure. Tensile tests in hydrogen atmosphere were carried out by TWI Ltd., using a specially designed vessel. This tensile test equipment for hydrogen atmosphere is shown in Fig. 3.3. This equipment is able to perform a tensile test at pressures of up to $100 \mathrm{MPa}$ and at temperatures between $-150{ }^{\circ} \mathrm{C}$ and $85^{\circ} \mathrm{C}$ [98]. Further details of the equipment are given in Ref. [99].

The tests were conducted at a constant displacement rate of $0.1 \mathrm{~mm} / \mathrm{min}$ resulting in an initial strain rate of $5.5 \times 10^{-5} \mathrm{~s}^{-1}$ according to ASTM-G142-98 for the testing of metals in hydrogen [100]. The tensile reduction of the area was measured ex-situ with a calliper. The Relative Reduction of Area (RRA) was calculated by dividing the reduction of area in hydrogen by the reduction of area in the air (see section 2.2.2). In addition, martensite formation during the tensile test was monitored. The martensite content was measured every $5 \%$ of applied engineering strain using a Feritscope ${ }^{\circledR}$ MP30 device (Helmut Fischer GmbH, Sindelfingen, Germany), by monitoring magnetic portion in the sample. This measurement was carried out in air at - $50^{\circ} \mathrm{C}$. 


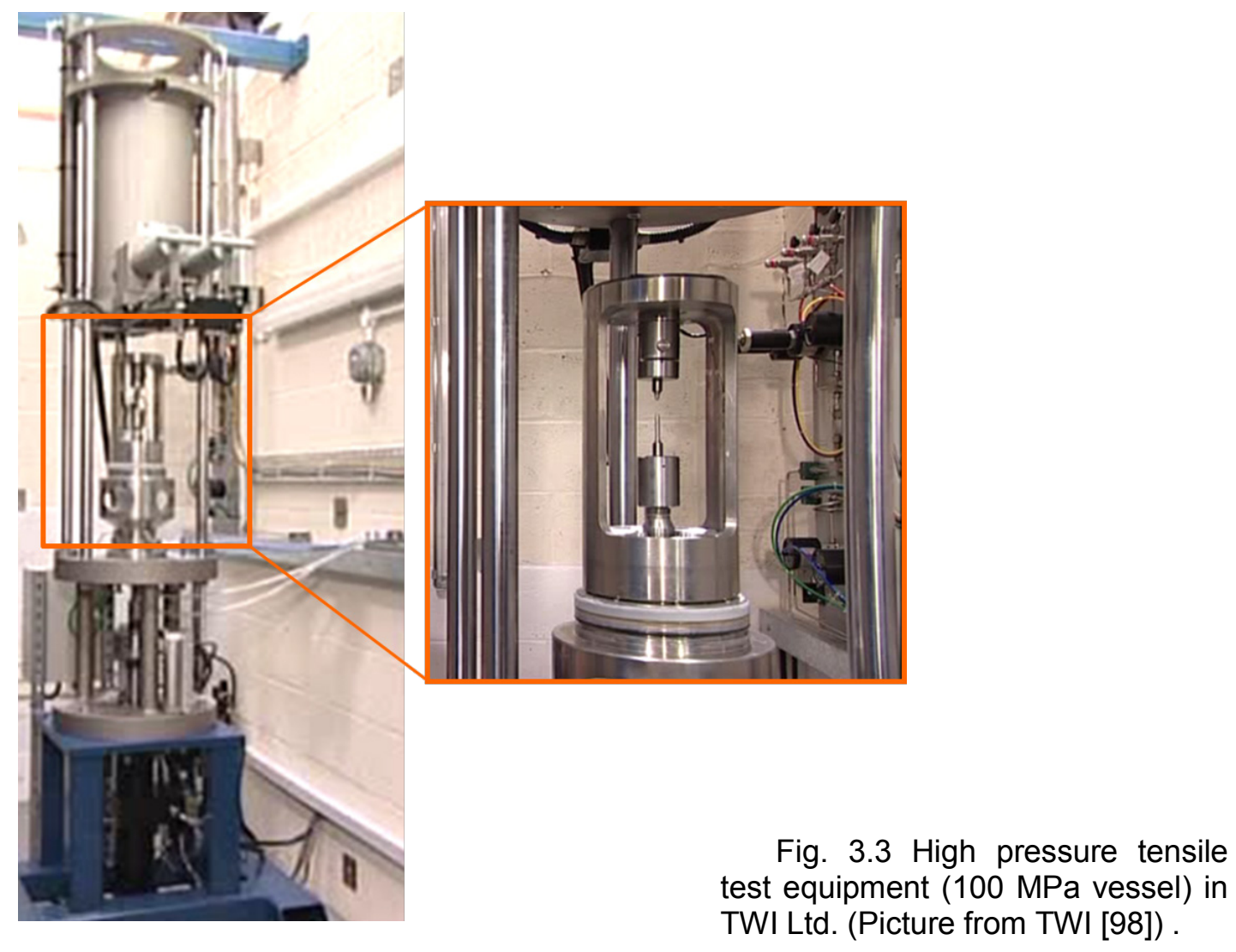

\subsection{Oxide layer analysis}

Secondary Ion Mass Spectrometry (SIMS) analysis (see 3.2.1) was conducted to investigate the impact of the heat treatment on the oxide layer. In case of W19, cross section analysis by EDX was also performed.

\subsubsection{Secondary Ion Mass Spectrometry (SIMS)}

Secondary Ion Mass Spectrometry (SIMS) enables to determine the atomic and molecular composition of a solid by sputtering the surface of the specimen with a high energy focused primary ion beam. A fraction of sputtered ion particles is electrically charged and analyzed according to their mass to charge ratio in a mass spectrometer. SIMS is available to obtain chemical information only from the near surface region because the emitted ions originate from the uppermost one or two monolayers bombarded surface. On the contrary, other analytical techniques such as Energy Dispersive 
Spectroscopy (EDS) and X-ray diffraction (XRD) give information from over several $\mu \mathrm{m}$ in depth. Hence, SIMS is used as a surface analysis technique such as X-ray Photoelectron Spectroscopy (XPS) and Auger Electron Spectroscopy (AES). The advantages and disadvantages of SIMS are briefly summarized below.

\section{Advantages}

- All elements detectable

- Isotope identification

- Low detection limit (ppm - ppb)

- Shallow information depth (1 - 2 monolayer)

- Chemical information (molecules, clusters)

- High depth resolution $(<1 \mathrm{~nm})$

\section{Disadvantages}

- $\mu \mathrm{m}$ level lateral resolution

- Poor quantification because secondary ion yields strongly depend on the matrix. For quantitative analysis, composition standards are essential.

- Flat surface required

- Destructive analysis

\subsubsection{Time of Flight Secondary Ion Mass Spectrometry (TOF-SIMS)}

SIMS can be broadly categorized into dynamic SIMS and static SIMS. The dynamic SIMS uses a high flux of primary ion beam to erode the surface rapidly and is appropriated for impurity analysis and bulk analysis (depth profiles) In contrast, the static SIMS employs a low flax of pulsed primary ion beam below the static limit $\left(<\sim 1 \mathrm{E} 12\right.$ ions $\left./ \mathrm{cm}^{2}\right)$ to obtain atoms, molecules, and large molecular fragments from e.g. organic compounds.

TOF-SIMS equipped with a pulsed primary ion beam and time of flight mass analyzer was developed as static SIMS by A. Benninghoven in the late 
1980s and this invention has later been developed to obtain depth profiles by combination of analysis beam and sputter beam. A detailed history of the development of TOF-SIMS can be found in the book edited by Vickerman [101].

TOF-SIMS IV (ION-TOF GmbH, Münster, Germany) used in this study is equipped with a 2-lens Ga gun for analysis and a combination of a $\mathrm{Cs}$ and an Ar sputter gun for sample erosion, as shown in Fig. 3.4 [102]. Both guns are mounted at an angle of $45^{\circ}$ to the sample.

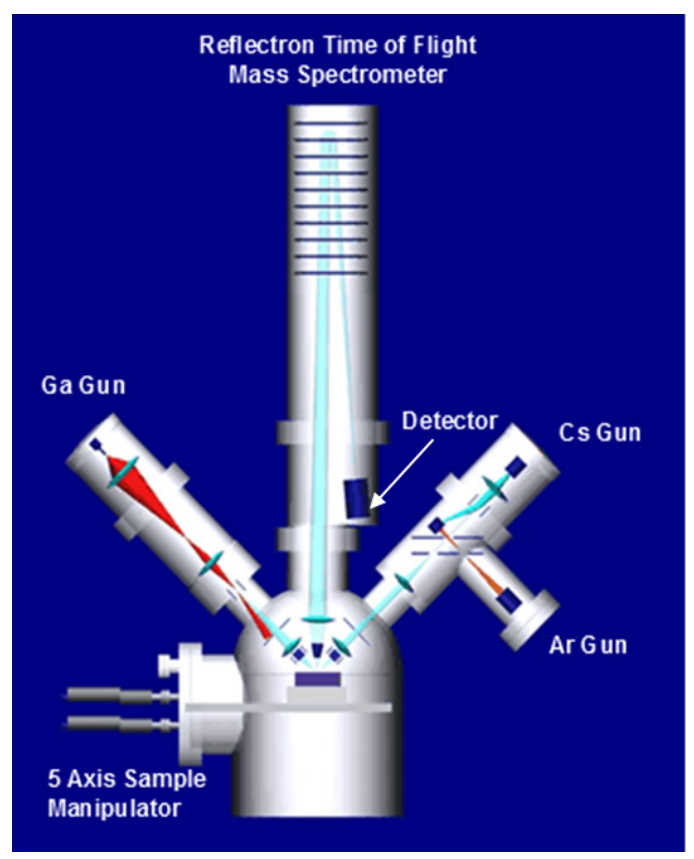

Fig. 3.4 Schematic drawing of TOF-SIMS used in this study. (Picture from ION-TOF GmbH [102])

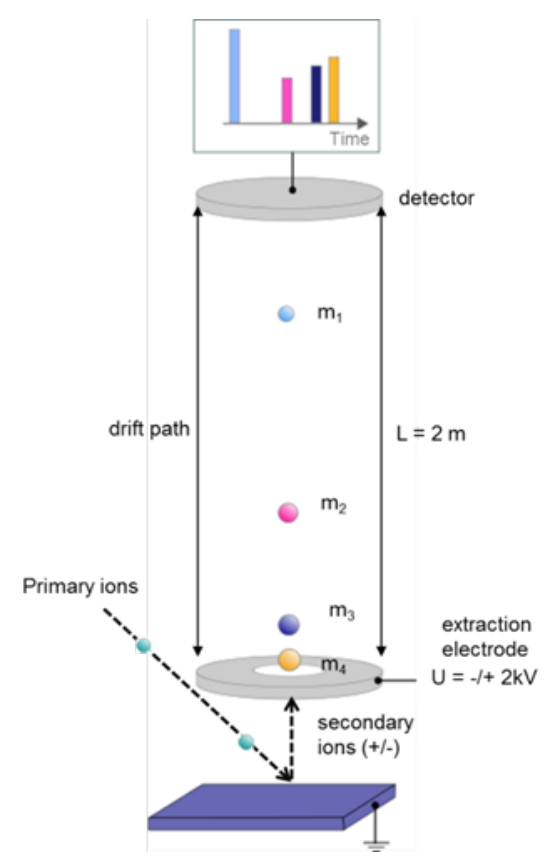

Fig. 3.5 Mass dispersion in a drift path. Time of flight is converted via Eq. (3-1) (Picture from ION-TOF $\mathrm{GmbH}$ [102], modified)

The sample is connected to ground potential. The secondary ions are extracted perpendicular to the sample surface with an extraction potential of $2 \mathrm{kV}$ and separated by a time of flight analyzer of the reflectron type with 2 $\mathrm{m}$ drift path according to their flight time. A schematic view of mass dispersion in a drift path is given in Fig. 3.5

According to the equation of kinetic energy, the measured flight time, $t$ 
can be converted to mass by using Eq. (3-1)

$$
t=L \sqrt{\frac{m}{2 z U}}
$$

where $L$ is a length of drift path, $m / z$ is ions of mass to charge ratio and $U$ is applied voltage to the extraction electrode.

The secondary ions are converted to the signals by a microchannel plate/scintillator/photomultiplier detector arrangement. Time-of-flight (t), position $(\mathrm{x}, \mathrm{y})$ and measurement time $(\mathrm{T})$ for each secondary ion are stored in the raw data file. This raw data file allows to reconstruct spectra, images and profiles after the measurement.

\subsubsection{Analysis Gun}

In this study, the $25 \mathrm{keV}$ gallium liquid metal ion gun (LMIG) was operated in two different modes, so called the bunched and the burst alignment mode as shown in Table 3.2. In short, the measurement must be compromised between pulse width (mass resolution) and spot size (lateral resolution).

In the bunched mode, $15-25 \mathrm{~ns}$ of ion packages are compressed to $0.7 \mathrm{~ns}$ pulses width by a buncher. The bunched mode allows high mass resolution without reducing the number of the primary ion current. TOF-SIMS spectrum around $\mathrm{m} / \mathrm{z} 60$ is shown in Table 3.2.. The bunched mode can resolve ${ }^{60 \mathrm{Ni}}\left(\mathrm{m} / \mathrm{z}\right.$ 59.93) and $\mathrm{SiO}_{2}(\mathrm{~m} / \mathrm{z}$ 59.97). However, the buncher introduces an energy spread of the beam. This results in a high chromatic aberration of the target lens. Therefore, the lateral resolution of the bunched mode is limited to several $\mu \mathrm{m}$.

The burst alignment mode is used for image analysis because of its good lateral resolution. However, the disadvantage of this mode is a low mass resolution, which results from long pulse of the analysis beam. The secondary ion image taken by the burst alignment mode in Table 3.2 is constructed from the broad peak at $60 \mathrm{~m} / \mathrm{z}$ which contains the signal of ${ }^{60} \mathrm{Ni}$ 
(m/z 59.93) and $\mathrm{SiO}_{2}(\mathrm{~m} / \mathrm{z} 59.97)$.

Table 3.2 Operation modes of Ga gun

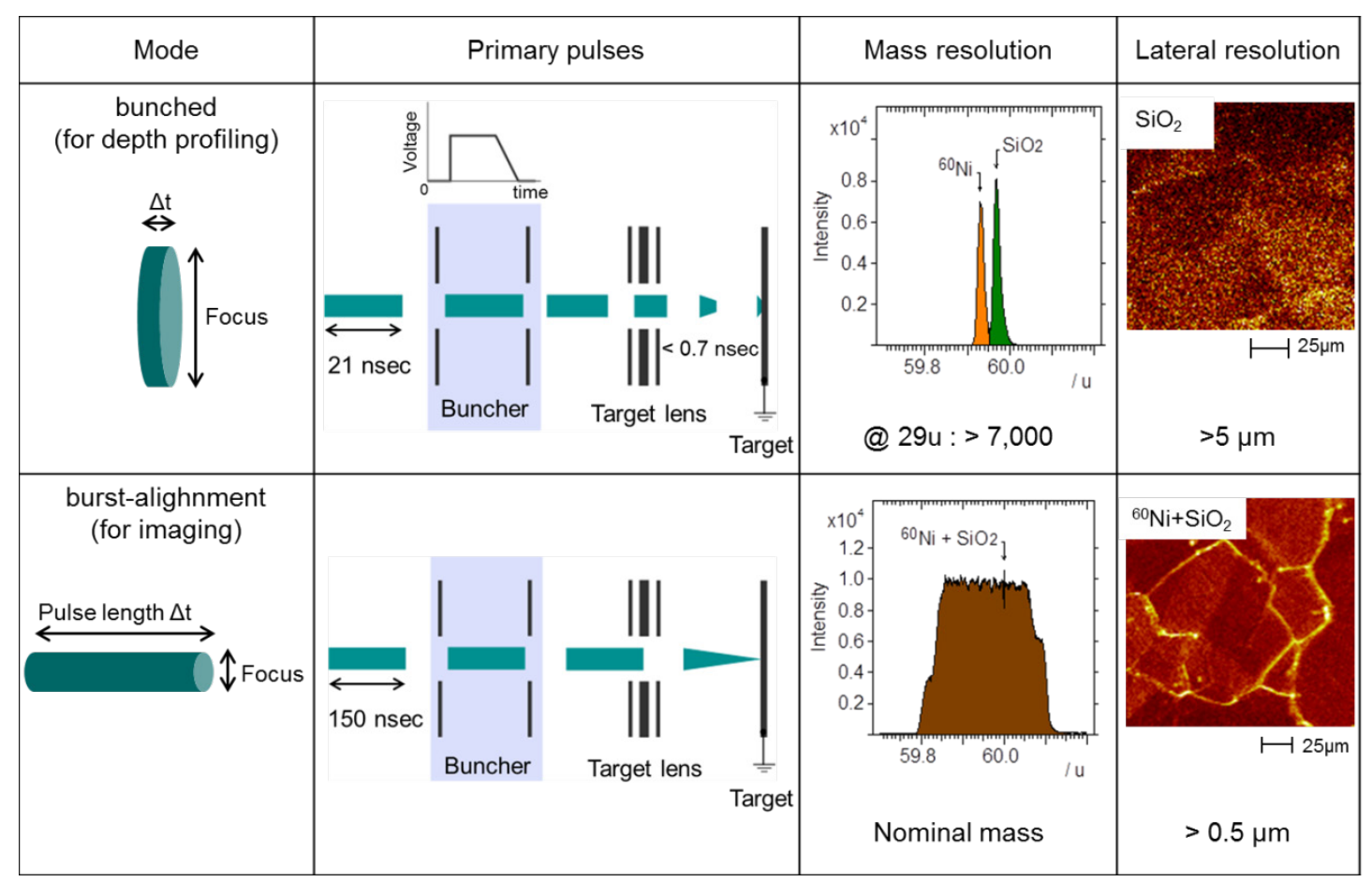

\subsubsection{Polarity}

When the analysis beam bombards a sample surface under ultra-high vacuum (UHV) conditions, secondary particles including electrons, photons, neutral atoms as well as positive and negative ions arise from the collision process between the primary ions and the sample atoms as well as by collisions between sample atoms. Either positive ions or negative ions are extracted perpendicular to the sample surface by applying negative or positive voltage between the sample and the extraction electrode as shown in Fig. 3.6 [102]. This means, that only one polarity canable to measure in one analysis batch. 
(a) Positive polarity

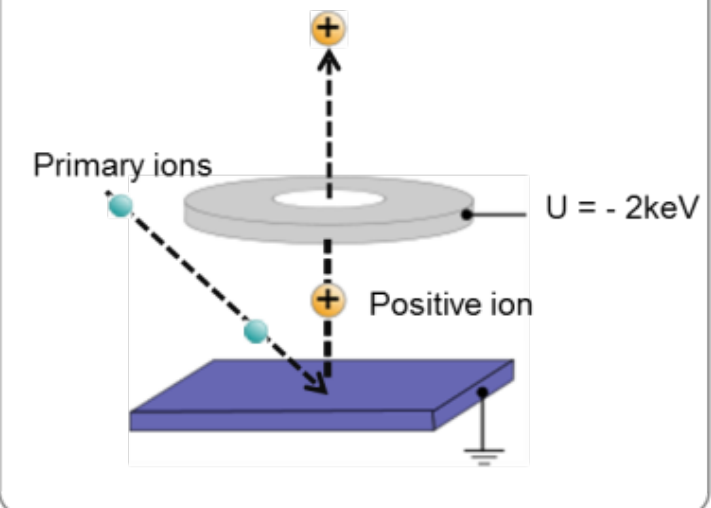

(b) Negative polarity

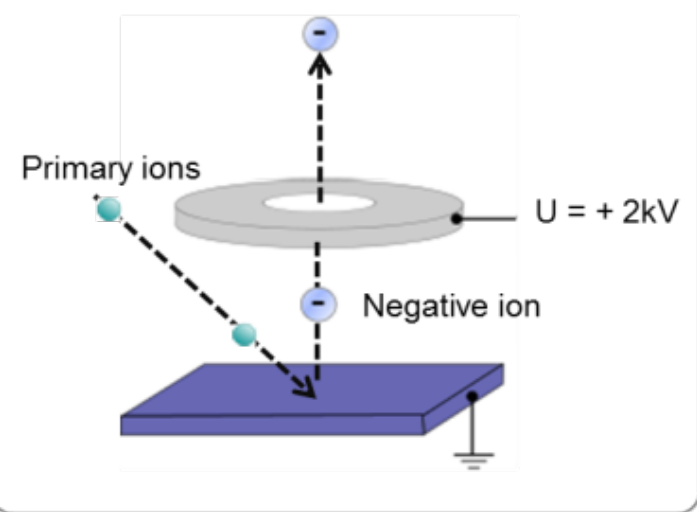

Fig. 3.6 Extraction electrode voltage for (a) positive polarity (b) Negative polarity (Picture from ION-TOF GmbH [102], modified)

The selection of polarity depends on the secondary ion yield, defined as the fraction of sputtered atoms that become ionized. The secondary ion yield is directly related to the ionization potential for positive secondary ions, and to the electron affinity for negative secondary ions. A simple thumb of the polarity selection for pure elements is indicated on the periodic table in Fig. 3.7 [103]. For analysis of oxide layers on metals, the negative polarity is generally used.

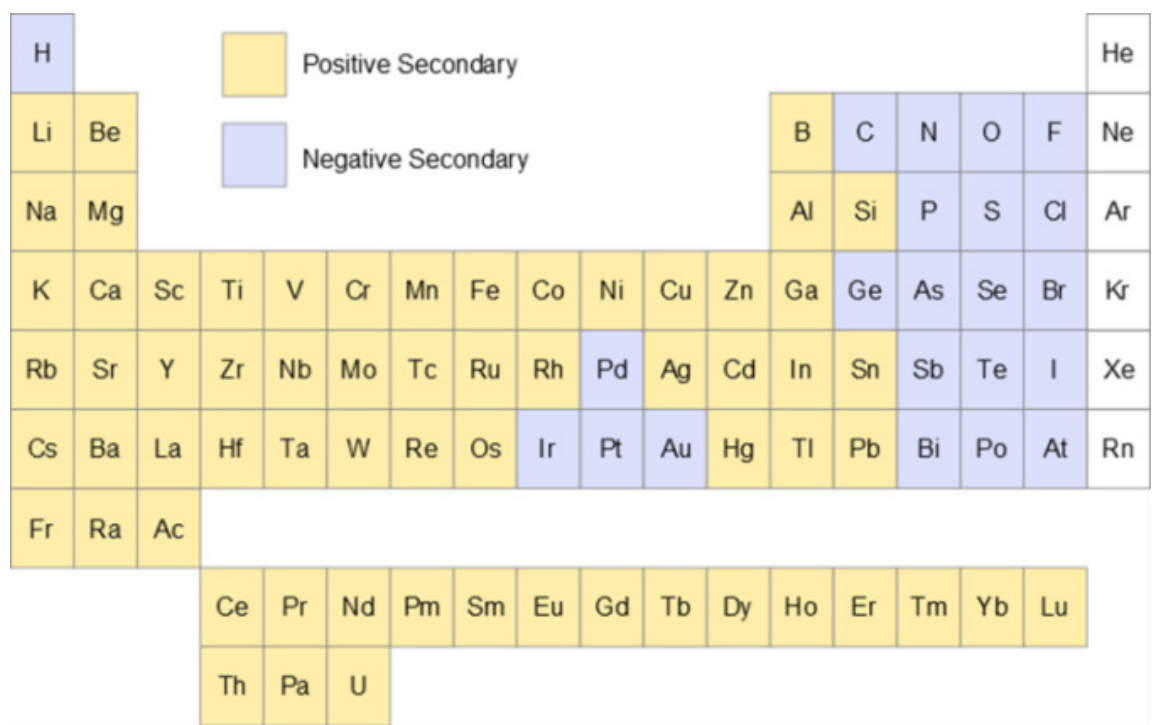

Fig. 3.7 Periodic table for the polarity selection based on Element information in TOF-SIMS software in ver. 4.1 [103]. 


\subsubsection{Dual Beam Technique for Depth Profiling}

In TOF-SIMS, quite low flux of the pulsed analysis beam is used for the observation of an uppermost monolayer of a solid, as described in 3.2.1.1. For depth profiling analysis, dual beam mode is used. The schematic of depth profiling experiment is shown in Fig. 3.8. In this mode, an additional ion beam (sputter beam) is operated over several hundred $\mu \mathrm{m}$ to erode the surface. An analysis beam is rastered within a central region of the sputtered crater. In consequence, the secondary ions emitted from the flat bottom of the crater area are analyzed.

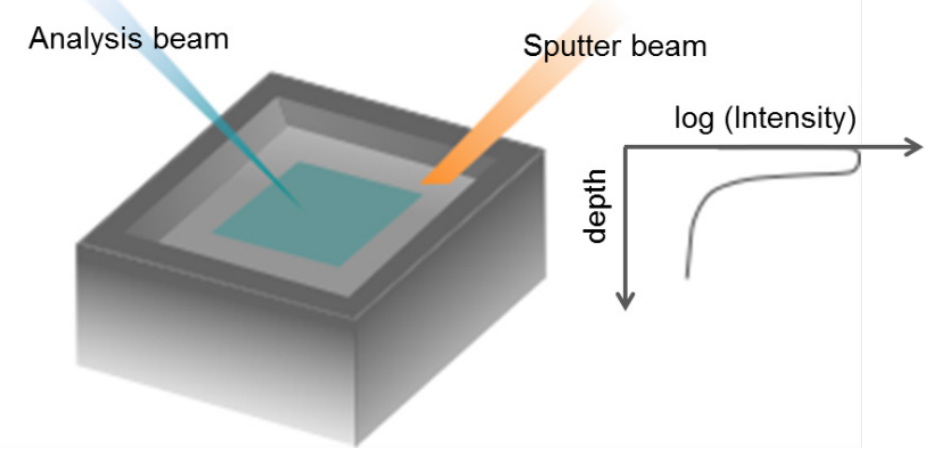

Fig. 3.8 A schematic of depth profiling by dual beam mode.

The depth profile analysis sequences are as follows; The analysis beam strikes the solid, then the extraction voltage of the analyzer can be applied for $5-10 \mu$ s to extract the secondary ions emitted from the surface. While secondary ions travel through the drift path for $100 \mu \mathrm{s}$, the extraction voltage can be switched off and the sputter beam can be switched on for the erosion. This cycle is repeated until the end of a measurement. More details of dual beam mode can be found in Ref. [104].

The sputter beam column is equipped with two different ion sources which are $\mathrm{Cs}^{+}$and either $\mathrm{O}^{+}$or $\mathrm{Ar}^{+}$. Secondary ion yields can be significantly increased by selecting specific sputter ion species. Bombardment with $\mathrm{O}_{2}{ }^{+}$ enhances the positive ion yield and $\mathrm{Cs}^{+}$enhances the negative ion yield [105]. In order to detect metal oxides signals, the negative polarity is selected here. Therefore, $\mathrm{Cs}^{+}$sputter beam has been mainly applied for the erosion in this 
study.

\subsubsection{Measurement conditions}

In this study, depth profiling and image depth profiling were mainly performed in negative polarity. A primary ion energy of $25 \mathrm{keV}$ with an ion current of $0.3 \mathrm{pA}$ was used for the analysis beam. A sputter ion energy of 1 $\mathrm{keV}$ with an ion current of $10 \mathrm{nA}$ was used for the $\mathrm{Cs}^{+}$beam. In the depth profiling mode, the analysis beam was optimized to the bunched mode and rastered over an area of $150 \times 150 \mu^{2}$, centered inside a $300 \times 300 \mu^{2}$ crater, sputtered by the $\mathrm{Cs}^{+}$sputter beam. Both beams were operated with an $128 \times 128$ pixel array. Image depth profiling was acquired with the burst alignment mode at an analysis area of $150 \times 150 \mathrm{\mu m}^{2}$ and with a $\mathrm{Cs}^{+}$sputter beam. An image resolution of $256 \times 256$ pixels was selected so that the finely focused primary ion beam hits the whole analysis area. Since the sputter rate of $\mathrm{Cs}^{+}$increases with increasing the image resolution, the sputter area of the $\mathrm{Cs}^{+}$beam was extended to $500 \times 500 \mu^{2}$ in order to obtain the comparable sputter rate to the resolution of $128 \times 128$ pixels in the depth profile mode. The images of $256 \times 256$ pixels were compressed to $128 \times 128$ pixels to increase the contrast by using the instrument software (ION-TOF GmbH, Münster, Germany) during data processing.

Fig. 3.9 shows the difference in (a) the $128 \times 128$ pixel image and (b) the compressed image from $256 \times 256$ pixels. Compressed images reveal more clear distribution of Si segregated along grain boundaries of stainless steel.

The measurements were carried out in ultra-high vacuum with a background pressure of $1 \times 10^{-7} \mathrm{~Pa}$ in order to prevent surface oxidation during the analysis. It is well known that the oxide layer thickness formed in air at room temperature is several $\mathrm{nm}$. Asami reported on a $4 \mathrm{~nm}$ oxide layer thickness formed on AISI 304 during wet polishing [106]. In this study, the oxide layer thickness of polished W11 obtained from depth profile result was set to $4 \mathrm{~nm}$, then the calculated sputter rate was applied to all samples, except for the semi-cylindrical shape samples. Therefore, the sputter depths given in this study are somewhat approximated. 


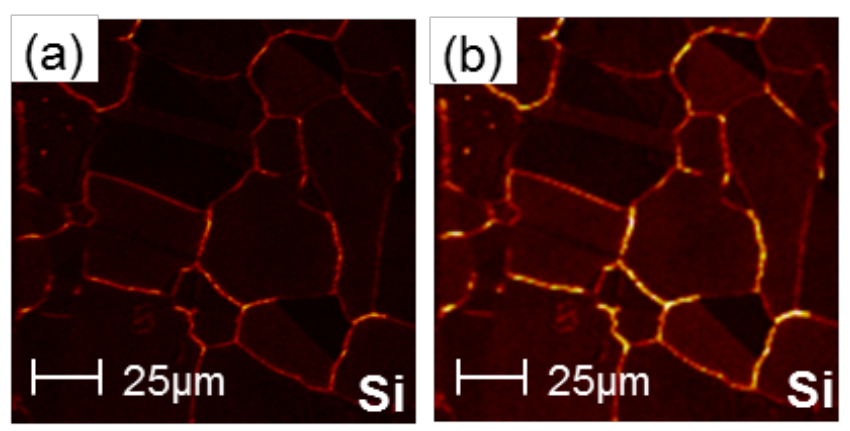

Fig. $3.9 \mathrm{Si}$ image (a) aquired with $128 \times 128$ pixels and (b) compressed from $256 \times 256$ pixels. The compressed image (b) shows the distribution of Si along grain boundaries of stainless steel with high contrast.

\subsubsection{Sample handling}

Inappropriate sample handling easily leads to sample contamination which affect the SIMS analysis. As an example; if plastic bags are used as sample containers, sample surface is inevitably contaminated by organics although they appear to be clean under an optical microscope. This contaminant often results in a crucial problem for surface analysis because TOF-SIMS is extremely sensitive and able to detect not only inorganics but also organics. For depth profile analysis of a flat surface sample, organic contamination is less problematic in this regard, since the contaminants are consumed in the course of erosion by sputter beam. However, it becomes critical for depth profiling analysis when the sample surface is rough or uneven.

In this section, the above mentioned aspects will be addressed in detail showing depth profiling results on the cylindrical and semi-cylindrical samples. Fig. 3.10 (a) shows an optical image of the cylindrical sample after tensile test. The sample surface has a very rough appearance and many transverse cracks resulting from tensile deformation. Fig. 3.10 (b) is the secondary electron image of the rectangular region highlighted in Fig. 3.10 (a). The enlarged secondary electron image verifies a wavy height modulation originating from the machining process to achieve the cylindrical shape. 


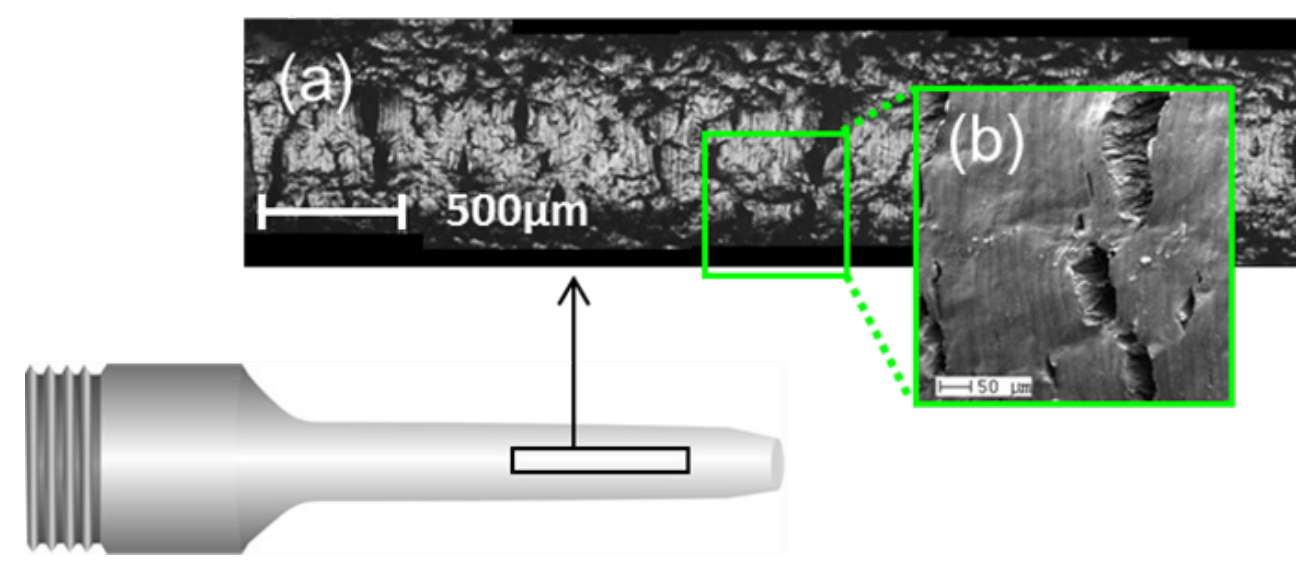

Fig. 3.10 Austenitic stainless steel sample after tensile test. (a) Optical image of the surface near necked region and (b) secondary electron image of the rectangular region shown in (a). Schematic tensile test sample indicated the observation area by optical microscope. The sample surface has a very rough appearance, showing several tens micro-size cracks due to plastic deformation. The enlarged secondary electron image verifies a wavy height modulation caused by the machining process to achieve the cylindrical shape.

Secondary ion images of the uppermost layer corresponding to the secondary electron image in Fig. 3.10 (b) are shown in Fig. 3.11 (a). Bright regions on the secondary ion image indicate a strong signal of the selected ion, while darker regions indicate a weaker signal. Fig. 3.11 (a) reveals inhomogeneous distribution of $\mathrm{Fe}^{+}, \mathrm{Cr}^{+}$and $\mathrm{Ni}^{+}$selected as main elements of stainless steel. $\mathrm{Si}_{2} \mathrm{C}_{5} \mathrm{H}_{15} \mathrm{O}^{+}$shows relatively homogeneous distribution and $\mathrm{C}_{3} \mathrm{~F}_{7}+$ distributes partially. Both of $\mathrm{Si}_{2} \mathrm{C}_{5} \mathrm{H}_{15} \mathrm{O}^{+}$and $\mathrm{C}_{3} \mathrm{~F}_{7}{ }^{+}$are fragment ions originated from two types of contamination, as described later.

To remove the contamination, sputter cleaning was performed by $1 \mathrm{keV}$ $\mathrm{Ar}^{+}$bombardment. Fig. 3.11 (b) indicates selected signals during sputter cleaning. The contamination by $\mathrm{Si}_{2} \mathrm{C}_{5} \mathrm{H}_{15} \mathrm{O}^{+}$and $\mathrm{C}_{3} \mathrm{~F}_{7}{ }^{+}$seems to be removed by this process since its signals are decreasing with increasing sputter depth. However, secondary ion image after sputter cleaning still confirms the contamination on surface especially at crack regions. Further sputter cleaning was continued until it passes through the oxide layer (about $7 \mathrm{~nm}$ in depth) as shown in Fig. 3.11 (d). After removing about $7 \mathrm{~nm}$ of surface atoms, high intensity regions of $\mathrm{Fe}^{+}, \mathrm{Ni}^{+}$and $\mathrm{Si}_{2} \mathrm{C}_{5} \mathrm{H}_{15} \mathrm{O}^{+}$as well as $\mathrm{C}_{3} \mathrm{~F}_{7}+$ are detected. Their positions are highly correlated (Fig. 3.11(e)),. $\mathrm{Si}_{2} \mathrm{C}_{5} \mathrm{H}_{15} \mathrm{O}^{+}$and 
$\mathrm{C}_{3} \mathrm{~F}_{7}{ }^{+}$are both contamination that hinder a proper determination of the elemental lateral distribution.

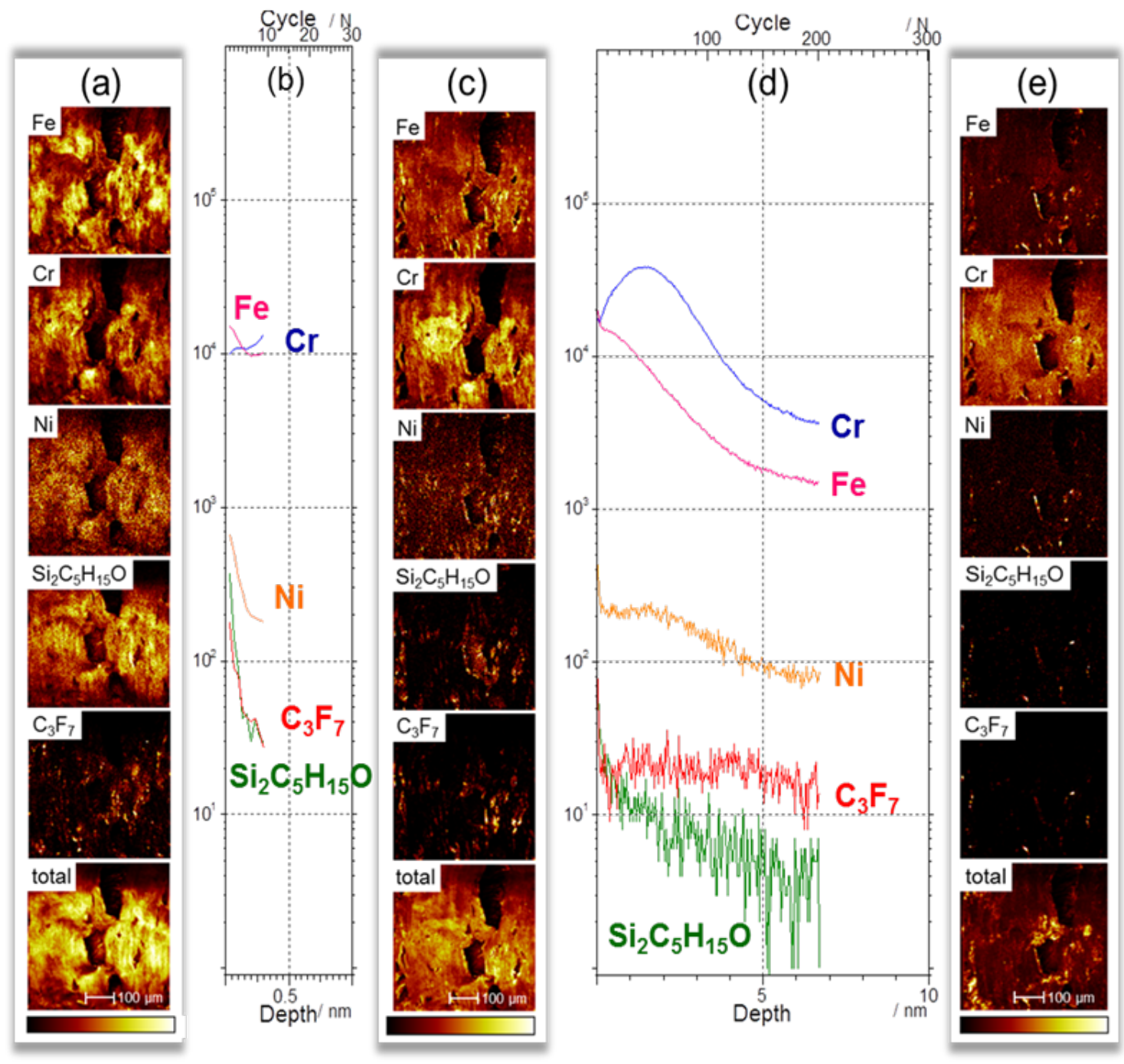

Fig. 3.11 Positive secondary ion images and depth profiling of an austenitic stainless steel sample after a tensile test. (a) uppermost layer, (b) secondary ion signal originated from contamination and main elements during removing uppermost layer (c) after removing the uppermost layer (at about $0.4 \mathrm{~nm}$ ), and (d) depth profile of oxide layer (e) after removing the oxide layer (at about $7 \mathrm{~nm}$ ). Color scale is normalized to maximum counts per pixel $(\mathrm{mc})$ for each individual ion. Contamination is visible at all sample depths, especially in crack regions.

As mentioned before, these polymer signals are not critical for depth profiling of a flat sample, because it is possible to remove them by the sputtering process. But here, although the sample was eroded by the $\mathrm{Ar}^{+}$ beam, contamination is still visible in Fig. 3.11 (c) and (e), especially at crack 
regions. This results from an uneven sputtering process, which is due to the high roughness of the surface. In addition, the secondary ion formation is strongly influenced by the local chemical state of the surface (matrix effect). Thereby the presence of the contaminations can increase the secondary ion yield, resulting in the high local brightness of the $\mathrm{Fe}^{+}, \mathrm{Cr}^{+}$and $\mathrm{Ni}^{+}$. These images strongly correlate with that of the contamination in Fig. 3.11 (e)

A detailed analysis reveals that the $\mathrm{Si}_{2} \mathrm{C}_{5} \mathrm{H}_{15} \mathrm{O}^{+}$signal represents polydimethylsiloxane (PDMS). This originates from the release agent of an adhesive tape that was used to fix the sample to measure its diameter. Further, PDMS is generally used as a mould-release agent in plastic bags. Plastic bags were used during the sample transfer. The $\mathrm{C}_{3} \mathrm{~F}_{7}{ }^{+}$signal represents perfluoropolyethers (PFPEs) arising from lubricant applied to screw part of the specimen before tensile test in order to assemble to tensile test machine. In fact, the $\mathrm{C}_{3} \mathrm{~F}_{7}{ }^{+}$signal is much stronger near the screw parts.

To observe the distributions of oxide and contamination, the negative secondary ion image was taken near the screw parts as shown in Fig. 3.12 (a). $\mathrm{F}^{-}, \mathrm{CF}_{3}{ }^{-}$and $\mathrm{COF}_{3}{ }^{-}$are fragment ions of PFPEs. The correlation between $\mathrm{FeO}^{-}$ and $\mathrm{O}^{-}$is observed while $\mathrm{F}^{-}$correlates inversely with $\mathrm{O}^{-}$. Inverse correlation between $\mathrm{F}^{-}$(red) and $\mathrm{O}^{-}$(blue) is clearly seen in the color overlay image shown in Fig. 3.12(b). This is attributed to the fact that the bright region of $\mathrm{O}$ indicates new surface formed during a tensile test since PFPEs is considered to have covered over the surface near the screw parts before the tensile tests. 


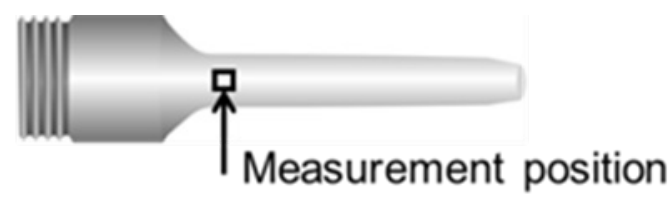

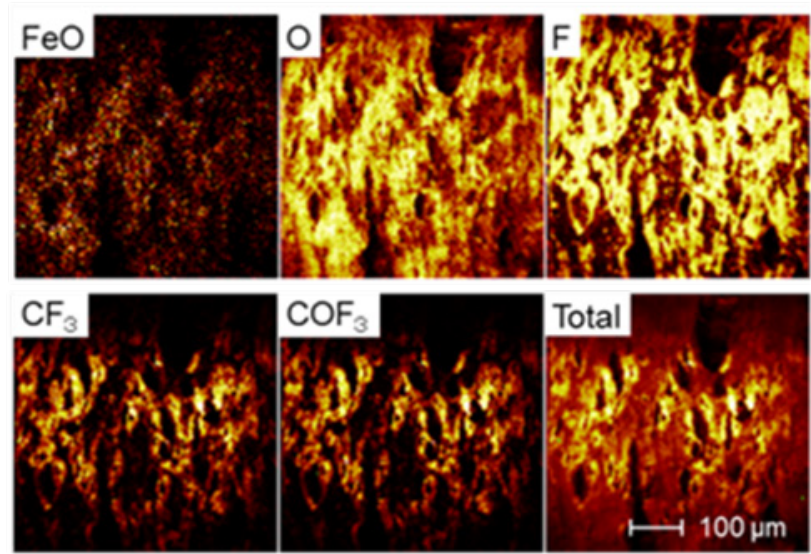

(a)

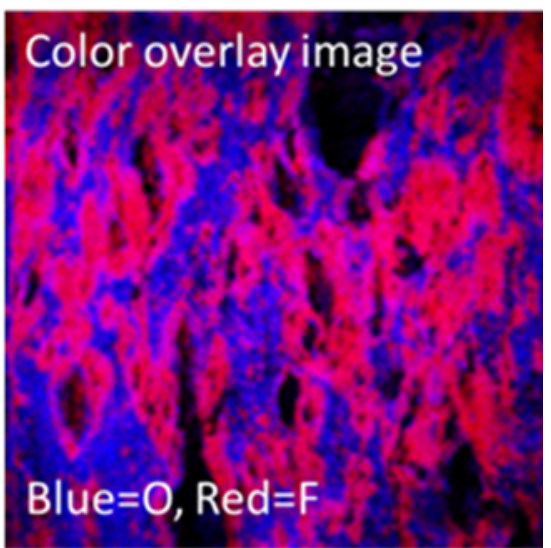

(b)

Fig. 3.12 (a) Negative secondary ion image taken near screw parts indicated in schematic tensile test specimen (b) Color overlay showing the different localizations of the $\mathrm{O}^{-}$and $\mathrm{F}^{-}$.

In order to obtain proper secondary ion images, the contamination must be removed. For this purpose the commercial austenitic stainless steels (AISI 304 grade) contaminated by PDMS and PFPEs were cleaned in appropriate chemical solutions [107] and were then analyzed by SIMS. Fig. 3.13 (a) shows positive secondary ion mass spectra acquired from AISI 304 contaminated by PDMS. The characteristic fragment ions, $\mathrm{Si}_{2} \mathrm{C}_{5} \mathrm{H}_{15}{ }^{+}$, $\mathrm{Si}_{3} \mathrm{C}_{5} \mathrm{H}_{15} \mathrm{O}_{3}{ }^{+}, \mathrm{Si}_{3} \mathrm{C}_{7} \mathrm{H}_{21} \mathrm{O}_{2}{ }^{+}$and $\mathrm{Si}_{4} \mathrm{C}_{7} \mathrm{H}_{21} \mathrm{O}_{4}{ }^{+}$, from PDMS are observed. After ultrasonic rinsing in hexane, these ions can be reduced significantly as shown in Fig. 3.13 (b). Similarly, Fig. 3.14 (a) shows positive secondary ion mass spectrum obtained from AISI 304 contaminated by PFPEs. The typical fragment ions, $\mathrm{C}_{2} \mathrm{~F}_{5}{ }^{+}, \mathrm{C}_{3} \mathrm{~F}_{5}{ }^{+}, \mathrm{C}_{3} \mathrm{~F}_{6}{ }^{+}$and $\mathrm{C}_{3} \mathrm{~F}_{7}{ }^{+}$, from PFPEs are confirmed. The signals from PTPEs decrease due to ultrasonic rinsing in methylene chloride as indicated in Fig. 3.14 (b). Thus, by applying this cleaning sequence, reliable elemental distributions can be obtained even on as-machined samples. 


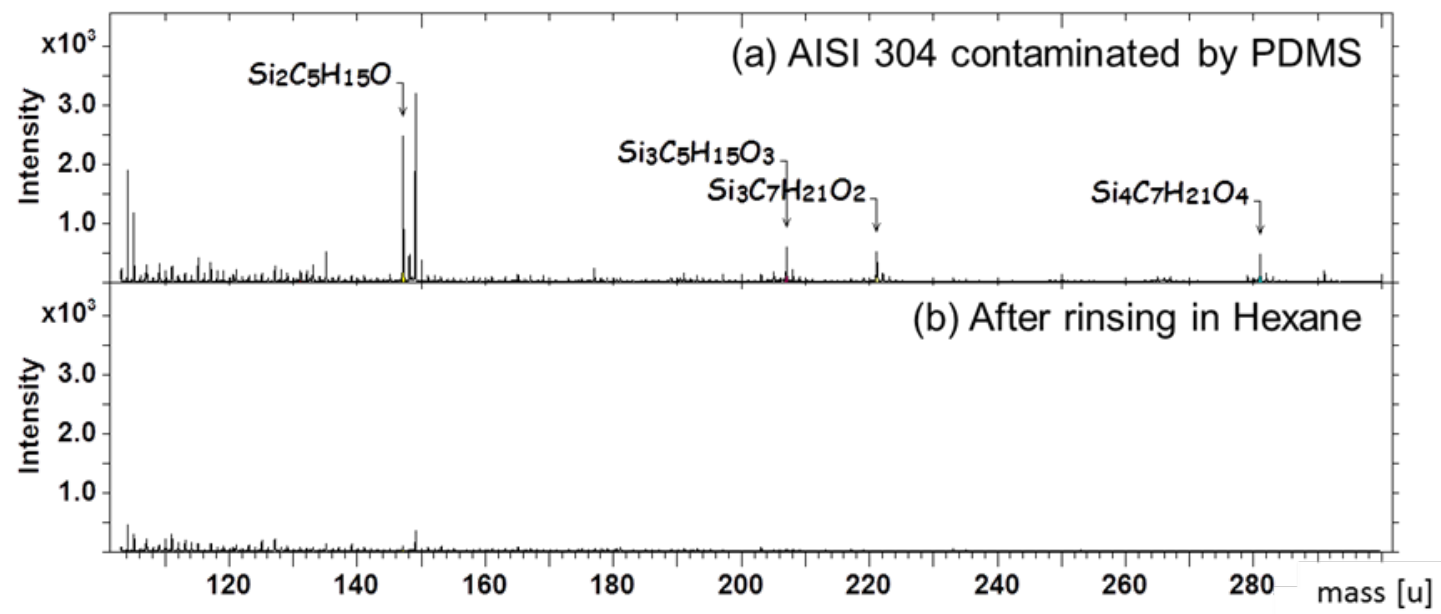

Fig. 3.13 Positive secondary ion spectrum on AISI 304 contaminated by PDMS (a) before and (b) after ultrasonic rinsing in n-Hexane. PDMS can be removed by rinsing in n-Hexane.

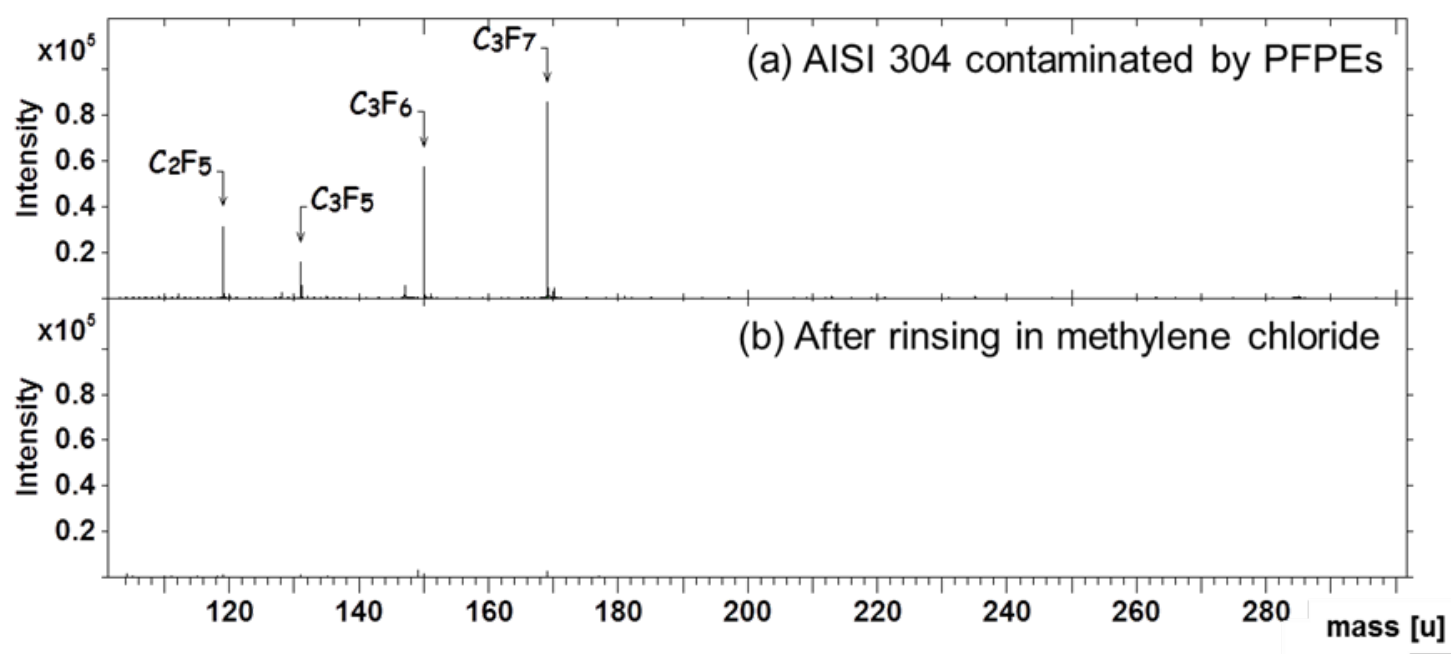

Fig. 3.14 Positive secondary ion spectrum on AISI 304 contaminated by PFPEs (a) before and (b) after rinsing in methylene chloride. PFPEs can be removed by ultrasonic rinsing in methylene chloride.

\subsubsection{Influence of the surface morphology on depth profile measurement by SIMS}

In general, SIMS requires a flat surface for precise depth profile analysis. However, for the investigation of the oxide layer on W11 with a'-martensite, which forms on the surface during the machining process, no further surface polishing is allowed in order to keep the surface in its original condition. The 
analysis surface, therefore, contains a certain curvature because of the cylindrical sample geometry. Further, the tensile test sample contains a wavy height modulation with a surface roughness of $R_{a}=0.38$ [20] originating from the machining process to achieve the cylindrical shape (Fig. 3.9). This wavy height modulation as well as the cylindrical sample geometry causes a degradation of the depth resolution during the SIMS analysis.

To reduce the degradation of the depth profiling on cylindrical sample resulting from the curvature and the wavy height modulation, attention is turned towards the scanning direction of the ion beams. There are two different ion beams used in SIMS: The sputter ion beam and the primary ion beam which are focused at the same position with an incident angle of $45^{\circ}$ to the sample surface. A curved sample results in a misalignment against both of the sputter and analysis ion beams. In the worst case, the erosion takes place apart from the analyses.

To avoid the misalignment of the two guns due to the cylindrical shape, a large sample diameter of $30 \mathrm{~mm}$ was selected for the SIMS measurement, whereas its diameter is $5 \mathrm{~mm}$ for tensile test. The cylindrical sample was cut to semi-cylindrical shape as shown in Fig. 3.15 (a). The wavy surface caused by the machining process is maintained. The optical image in Fig. 3.15 (b) shows the analytical surface which maintained the height modulation of about $50 \mu \mathrm{m}$ wavelength.
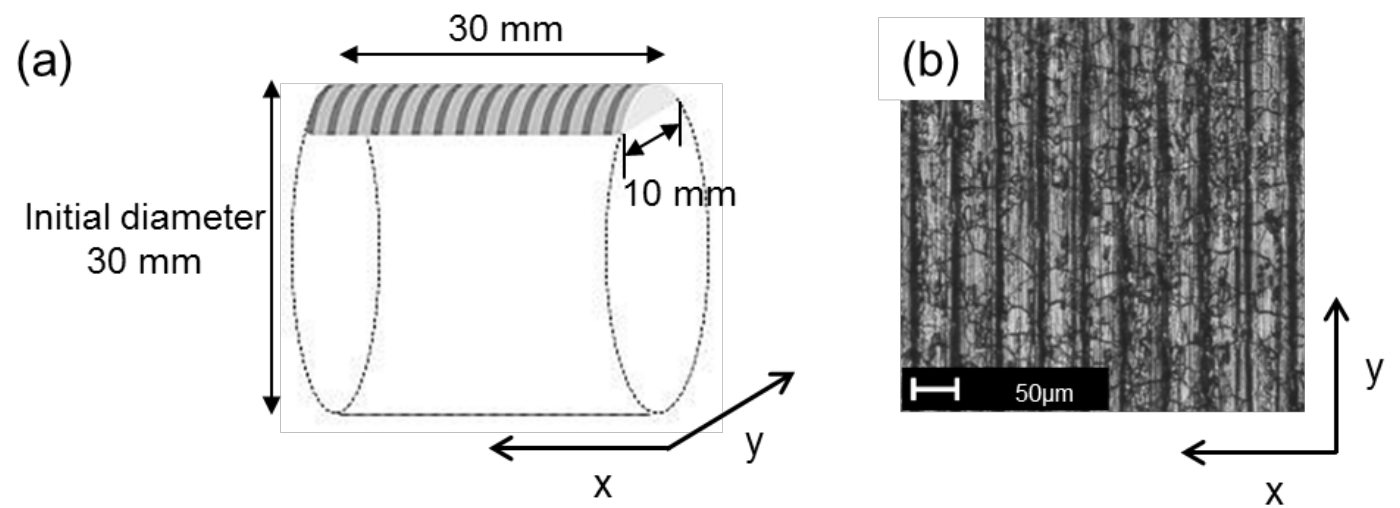

Fig. 3.15 (a) Sample geometry and (b) optical image of the sample. The wavy height modulation originating from the machining process is observed in optical image of the sample. 
Machining also results in surface contamination that has been removed by sample washing in different chemical solutions prior to the SIMS measurements, as described in Section 3.2.1.6.

Fig. $3.16(b-1)$ and (b-2) show the influence of the surface modulation and curvature on the depth resolution in the SIMS profiles. For purpose of comparison, the depth profile on an ideal flat surface is also shown in Fig. 3.16 (a). The related CCD camera images of the SIMS are depicted on each profile. These images verify the modulation orientation during the SIMS measurement which is vertical to the scanning direction $(b-1)$ and parallel to the scanning direction $(b-2)$. Here, instead of depth scale, the cycle number is used as $\mathrm{x}^{-}$-axis in order to evaluate depth resolution. Vertical red dotted lines indicate the interface between oxide layer and bulk in each profile.

Fig. 3.16 (a) shows the sharp profile of $\mathrm{Fe}^{+}$and $\mathrm{Cr}^{+}$and better signal to noise ratio when compared with profiles obtained on semi-cylindrical samples $((b-1)$ and $(b-2))$. Thus, the semi-cylindrical sample shape leads to not only the degradation of the depth resolution, but also a reduction of the signal to noise ratio. As can be seen, the broadened depth profiles of $\mathrm{Fe}^{+}$and $\mathrm{Cr}^{+}$appear for the vertical sample orientation in $(\mathrm{b}-1)$ compared to the parallel sample orientation in (b-2). The vertical red dotted lines mark similar features in both figures, visible at around 400 cycles (b-1) and at around 200 cycles $(b-2)$, respectively.

This finding is directly related to the surface roughness. In the vertical direction, erosion sputtering is pronounced on one side of the modulations, whereas analyze sputtering takes place at the opposite side of the modulation. This effect blurs the depth profile. In the parallel direction, erosion and analysis sputtering are possible on the hills and in the valleys of the wavy height modulation. Therefore, the depth profile is sharper and gives the "true" depth profile of the sample surface with higher accuracy.

Still, the height modulation widens the depth profile. This effect cannot be prevented when studies on as-machined samples are required. However, surface roughness effects can be minimized by choosing the orientation parallel to the scanning direction. 
(a) Flat sample

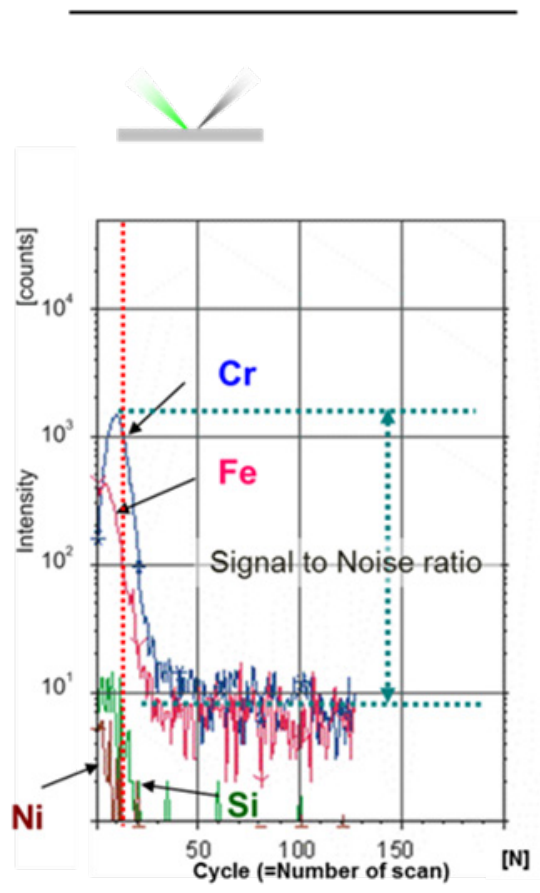

(b) Semi-cylindrical sample

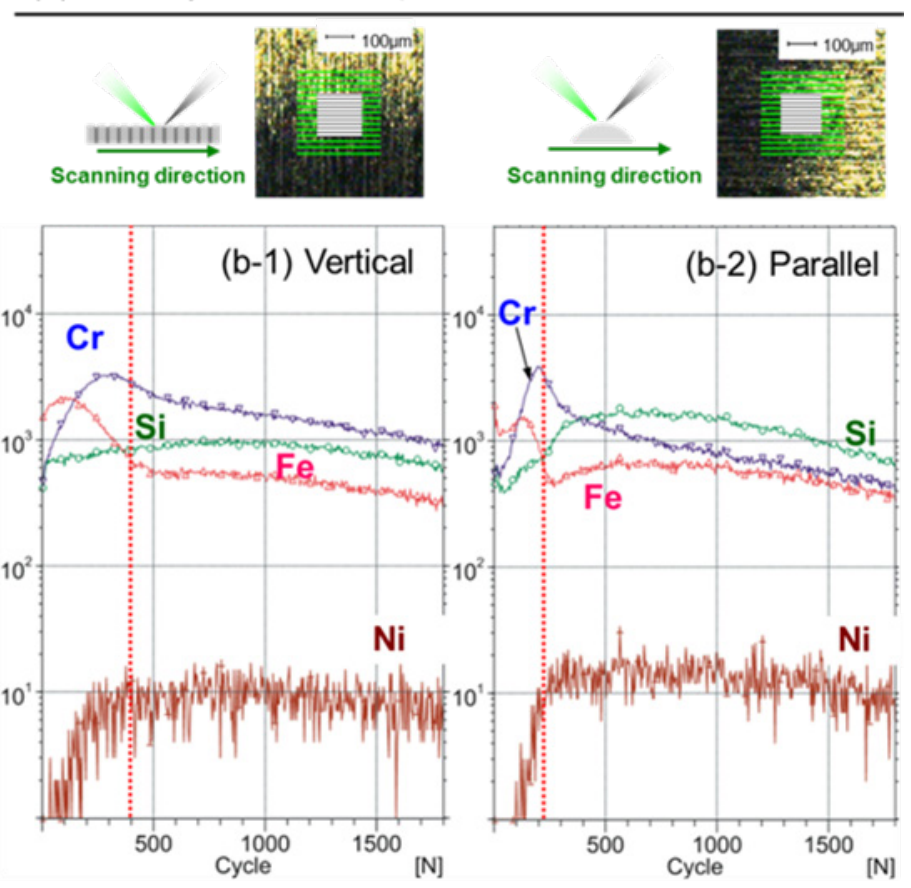

Fig. 3.16 Depth profiling on W11 (a) a flat surface and (b) semi-cylindrical shape with a wavy height modulation. (positive polarity). The profile strongly depends on the sample orientation, which is (b-1) vertical to surface roughness and (b-2) parallel to surface roughness. The scanning direction is indicated by green and black lines in CCD camera images shown in the top of each profile. The depth resolution is better for the parallel orientation.

\subsubsection{SIMS - Sample preparation}

For the SIMS analysis, several types of specimens were prepared. The shape of the specimen, the type of the furnace for the solution annealing, and solution annealing duration were changed according to the purpose. The samples were prepared, including solution annealing process, by Bochum University.

\subsubsection{Evaluation of oxide layer formed by three types of furnace}

For the selection of the applicable furnace, three types of vacuum furnaces equipped with, (a) an inductive heater, (b) a molybdenum heater, 
and (c) a graphite heater, were used for the solution annealing process. The sample shape, the annealing condition and the used furnace are listed in Table 3.3.

Table 3.3 Sample information for oxide layer evaluation formed by three types of furnace.

\begin{tabular}{cccccc}
\hline & Sample & & & \multicolumn{3}{c}{ Solution annealing condition } \\
\cline { 5 - 6 } \cline { 5 - 6 } Internal No. & Shape & & $\begin{array}{c}\text { Temp } \\
\left({ }^{\circ} \mathrm{C}\right)\end{array}$ & $\begin{array}{c}\text { Time } \\
(\mathrm{min})\end{array}$ & furnace \\
\hline W11 & disk & & 1050 & 15 & Inductive heater \\
W11 & disk & & 1050 & 30 & Inductive heater \\
W11 & disk & & 1050 & 60 & Inductive heater \\
W11 & disk & & 1050 & 15 & Mo furnace \\
W12 & disk & & 1050 & 15 & Mo furnace \\
W20 & disk & 1050 & 15 & Mo furnace \\
W11 & block & 1050 & 15 & Graphite furnace \\
\hline
\end{tabular}

\subsubsection{Influence of $\mathrm{a}^{\prime}$-martensite}

To characterize the oxide layer formed during the machining process, no further surface polishing is allowed in order to keep the surface in its original condition. As a result, the sample geometry for the SIMS analysis is semi-cylindrical as resulting from the tensile tested rods. The sample diameter of $30 \mathrm{~mm}$ was selected for SIMS measurement. Two semi-cylindrical samples were cut out from the cylindrical shape piece as illustrated in Fig. 3.17.

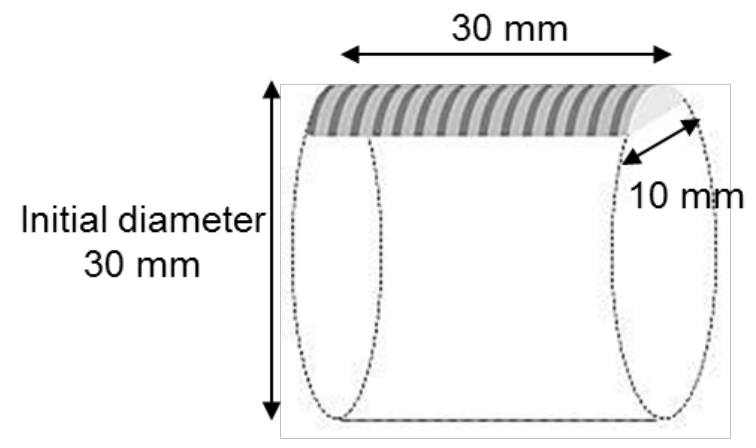

Fig. 3.17 Schematic illustration of sample geometry for W11 with and without $\alpha^{\prime}$ martensite 
One of them was annealed for $15 \mathrm{~min}$ at $1050{ }^{\circ} \mathrm{C}$ in a vacuum furnace equipped with a molybdenum heater after machining while another one was in machined condition.

\subsubsection{Influence of annealing time}

The oxide layer characterization was performed on W11 annealed for 15 $\min$ and for $30 \mathrm{~min}$. These samples were solution annealed at $1050{ }^{\circ} \mathrm{C}$ in graphite heater. In order to confirm the influence of solution annealing process on oxide layer formation, polished W11 was also measured by SIMS. The polishing was conducted with $3 \mu \mathrm{m}$ diamond suspension followed by final polishing with colloidal silica.

\subsubsection{Oxide layer characterization of low-nickel steels}

For the oxide layer characterization of low-nickel steels, all steels were the solution annealed for $30 \mathrm{~min}$ at either $1050{ }^{\circ} \mathrm{C}$ or $1150{ }^{\circ} \mathrm{C}$ by using graphite furnace. The solution annealing conditions are listed in Table 3.4.

Table 3.4 Sample information for oxide layer characterization of low-nickel steels

\begin{tabular}{ccccccc}
\hline \multicolumn{3}{c}{ Sample } & & & \multicolumn{3}{c}{ Solution annealing condition } \\
\cline { 1 - 1 } Internal No. & Shape & & $\begin{array}{c}\text { Temp } \\
\left({ }^{\circ} \mathrm{C}\right)\end{array}$ & $\begin{array}{c}\text { Time } \\
(\mathrm{min})\end{array}$ & furnace \\
\hline W11-W19 & block & & 1050 & 30 & Graphite furnace \\
W20, W21 & block & & 1150 & 30 & Graphite furnace \\
\hline
\end{tabular}

In case of W19, the cross section was characterized also by EDX. For the EDX analysis, a piece of W19 was cut by spark erosion machine. The cross section was polished down to mirror surface with $3 \mu \mathrm{m}$ diamond suspension, and with colloidal silica for finishing. 


\subsubsection{Energy Dispersive X-ray spectrometry (EDX)}

A cross sectional analysis on W19 was conducted by EDX in order to find out the interface, which cannot be achieved by SIMS. EDX analysis was peformed by FEI NOVA NANOSEM 650. The measurement was done with the help of M. Hahn. Details on EDX analysis can be found in Ref. [108].

\subsection{Dislocation observation and dislocation density measurement}

The observation of dislocation by transmission Electron Microscope (TEM) was performed only on W11 while the dislocation density measurements by X-ray diffraction (XRD) were conducted on all samples as shown in Table 3.5. Again, the disk shaped samples were prepared, including solution annealing process, by Bochum University. The measurements were conducted at Institut für Materialphysik (IMP), Universität Göttingen. The original sample dimension is $12 \mathrm{~mm}$ diameter with $1 \mathrm{~mm}$ thickness. The observed samples and their annealing conditions are also summarized in Table 3.5.

Table 3.5 Sample information for dislocation measurements by TEM and XRD.

\begin{tabular}{cccccc}
\hline & \multicolumn{3}{c}{ Solution annealing condition } & & \\
\cline { 2 - 4 } Internal No. & $\begin{array}{c}\text { Temp } \\
\left({ }^{\circ} \mathrm{C}\right)\end{array}$ & $\begin{array}{c}\text { Time } \\
(\mathrm{min})\end{array}$ & furnace & TEM & XRD \\
\hline W11 (as machined) & - & - & - & $\checkmark$ & $\checkmark$ \\
W11 & 1050 & 15 & Mo furnace & $\checkmark$ & $\checkmark$ \\
W11 & 1050 & 30 & Mo furnace & $\checkmark$ & $\checkmark$ \\
W11-W19 & 1050 & 30 & Graphite furnace & & $\checkmark$ \\
W20, W21 & 1150 & 30 & Graphite furnace & & $\checkmark$ \\
\hline
\end{tabular}

\subsubsection{Transmission Electron Microscope (TEM)}

A Transmission Electron Microscope (TEM) observations were carried out in collaboration with M. Deutges. TEM was performed with Philips CM 
12 at an acceleration voltage of $120 \mathrm{keV}$.

In the TEM, a focused electron beam (incident beam) hits an ultra-thin specimen, and electrons emerged from the exit surface are used to study the internal structure of the specimen. Now we consider a crystal containing an edge dislocation as a specimen (Fig. 3.18) [109].

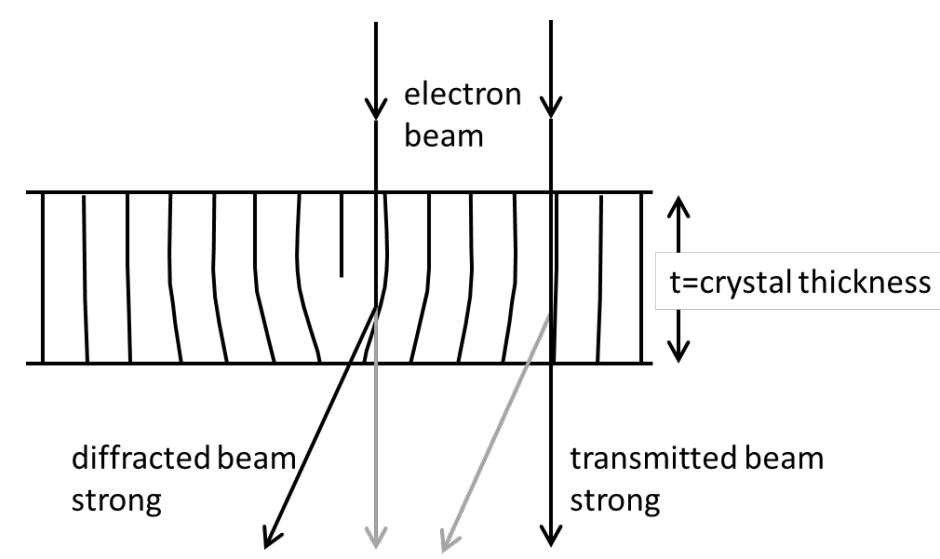

Fig. 3.18 Schematic illustration of diffraction around a dislocation core. (Picture from Putnis [109] )

In this case, the orientation of the lattice planes around the dislocation core is slightly distorted. Such local distortions alter the diffraction conditions. The electron beam is diffracted more strongly by the distorted lattice planes. In other words, the transmitted beam is weak in the vicinity of the dislocation core, while the transmitted beam is strong in the undistorted parts of the crystal. Such spatial distribution of transmitted electrons can be observed as contrast in images of specimen. In a bright-field image, the dislocations appear as black lines on a bright background [109].

The dislocation line may also appear and disappear by tilting the specimen during the observation, because the angle of incidence between the electron beam and the lattice planes are altered. Fig. 3.19 shows an atomic arrangement of an edge dislocation in different projections [110]. 

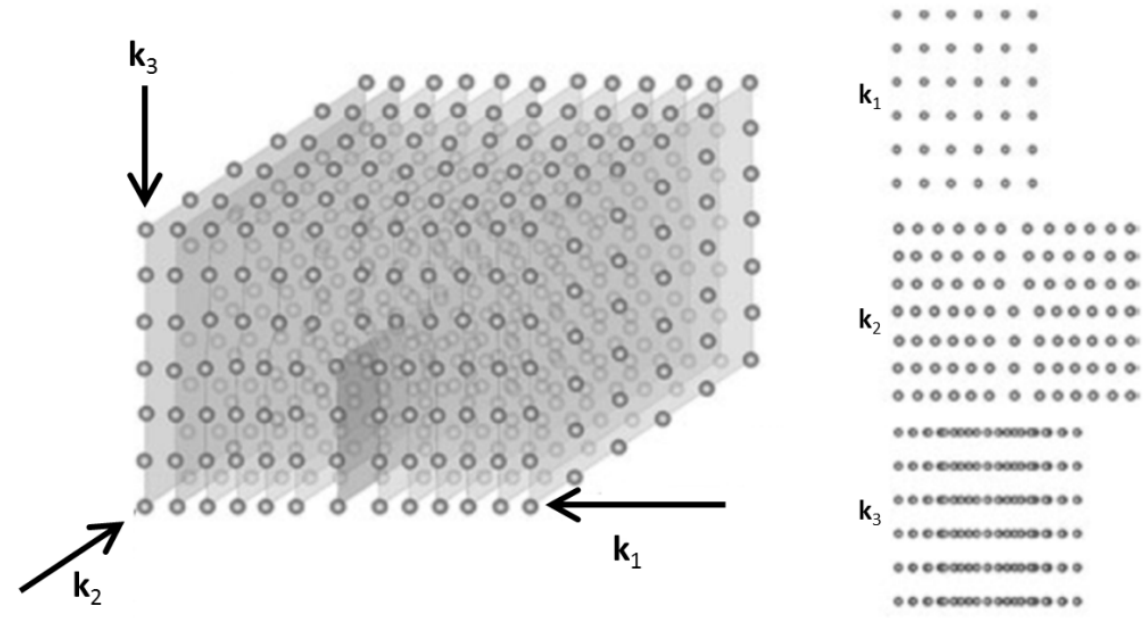

Fig. 3.19 Atomic arrangement of an edge dislocation in different projections. (Picture from Thomas and Gemming [110])

Again, the lattice planes around the edge dislocation is locally distorted. In directions $\mathbf{k}_{1}$ and $\mathbf{k}_{2}$, these are relatively less distorted and do not alter the projected picture, while the shift of the atoms is visible in $\mathbf{k}_{3}$ direction. Thus, in normal TEM, the dislocation line can be visible only when the incident electron beam is aligned with $\mathbf{k}_{3}$ direction. This means that TEM images shown in this study do not ehibit all dislocations in the analyzed area. The visibility of dislocations may be altered by tiliting the specimen, since different planes can be put into the diffraction condition.

\subsubsection{TEM - Sample preparation}

The $3 \mathrm{~mm}$ disks were cut from the samples with a diameter of $12 \mathrm{~mm}$ and thickness of $1 \mathrm{~mm}$. The sampling position is at $1 \mathrm{~mm}$-distance from the edge in order to eliminate the influence of a'-martensite introduced by the wet turning process (section4.2.1). The cutting process was conducted using spark erosion machine by F. Oelkers-Hilker. The sample preparation was conducted by M. Deutges. Initial thinning was conducted by mechanical polishing. Then dimpling and Ar ion beam milling was carried out in order to thin the center of the disk. The entire procedure is schematically illustrated in Fig. 3.20. 


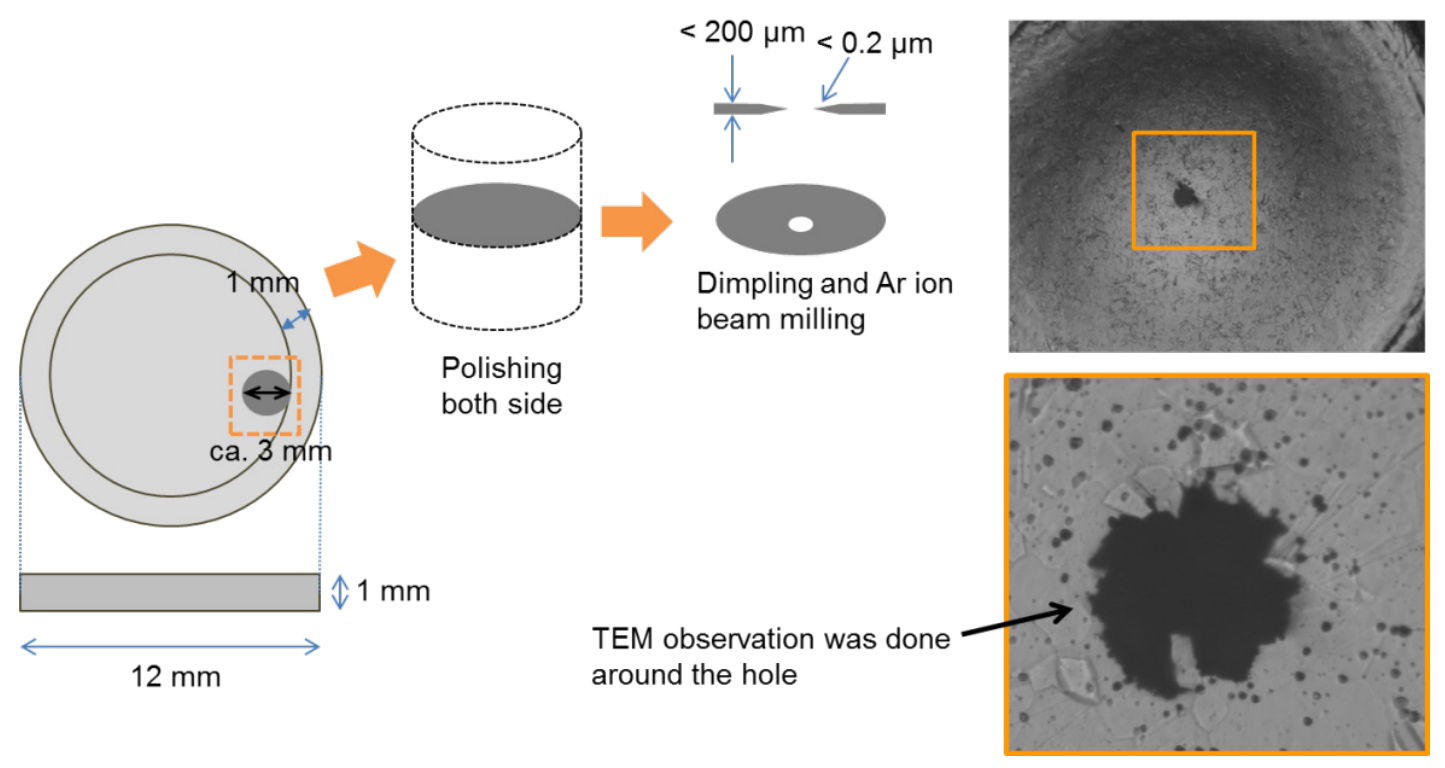

Fig. 3.20 Sample preparation procedure for TEM specimen

\subsubsection{X-ray diffraction (XRD)}

$\mathrm{X}$-ray diffraction (XRD) peak broadening caused by strain has been investigated in association with dislocation density in a solid. For each sample after annealing XRD peak broadening measurement was conducted by X'pert MRD (Philips, Co Ka).

A diffraction peak from unstrained crystallite appears at the position reflected equilibrium interplanar distance $d$, as illustrated in Fig. 3.21 (a). If uniform compressive stress is applied to grain, $d$ ' spacings become smaller than $d$, and the corresponding diffraction peak shifts toward high angles as shown in Fig. 3.21 (b). If several spacings randomly distribute due to inhomogeneous strain which is induced by the defects: dislocations, interstitials, vacancies, and stacking faults, these regions cause various peaks, each slightly displaced from the other, indicated in Fig. 3.21 (c) by the dotted curves. Therefore, peak broadening shown by the full curve can be observed resulting from the sum of the peaks [111]. 

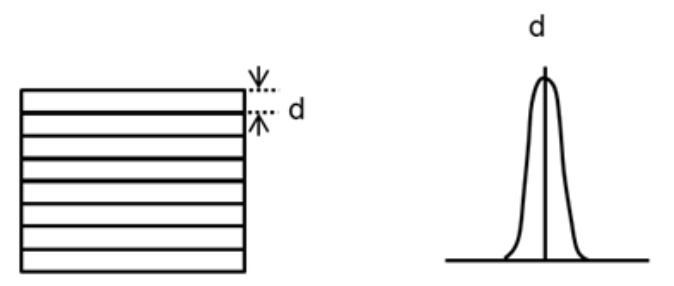

(a) unstrained crystallite
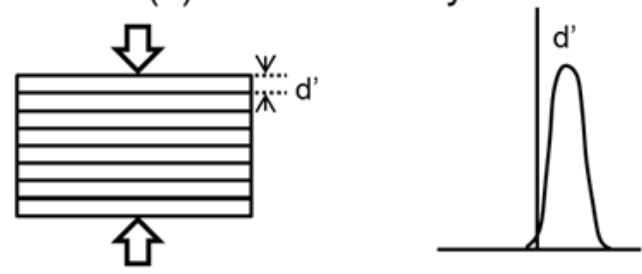

(b) homogeneously strained (compressed) crystallite
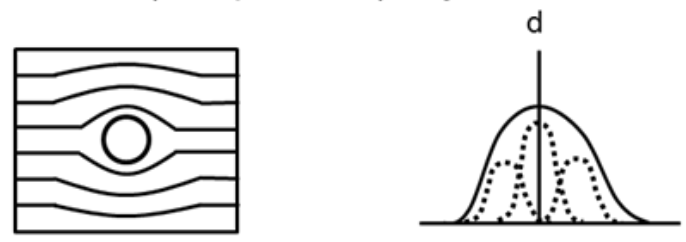

(c) inhomogeneously strained crystallite

Fig. 3.21 Effect of strain on peak width and position (a) unstrained crystallite (b) homogeneously strain crystallite and (c) inhomogeneously strained crystallite. XRD peak broadening by the full curve appears resulting from the sum of peaks by the dotted curves. (Picture from Cullity [111], modified)

The relationship between peak broadening and strain is given by Williamson-Hall (WH) equation [112] as follows.

$$
\beta_{t}\left(\frac{\cos \theta}{\lambda}\right)=\varepsilon\left(\frac{2 \sin \theta}{\lambda}\right)
$$

where $\beta_{t}$ is the full width at half maximum (FWHM) of a Bragg reflection excluding instrumental broadening, $\theta$ is the Bragg's angle, $\lambda$ is the wave length of X-ray, and $\varepsilon$ is the strain component. In this study, the diffraction peaks of (111), (110), (220), (311) and (222) were recorded and fitted with Lorentzian functions to obtain $\beta_{t}$. By plotting $\beta_{t}(\cos \theta / \lambda)$ versus $2 \sin \theta / \lambda$, the strain component can be obtained from the slope and this plot is known as a WH plot. Fig. 3.22 shows WH plot on W12 specimen as one example. 


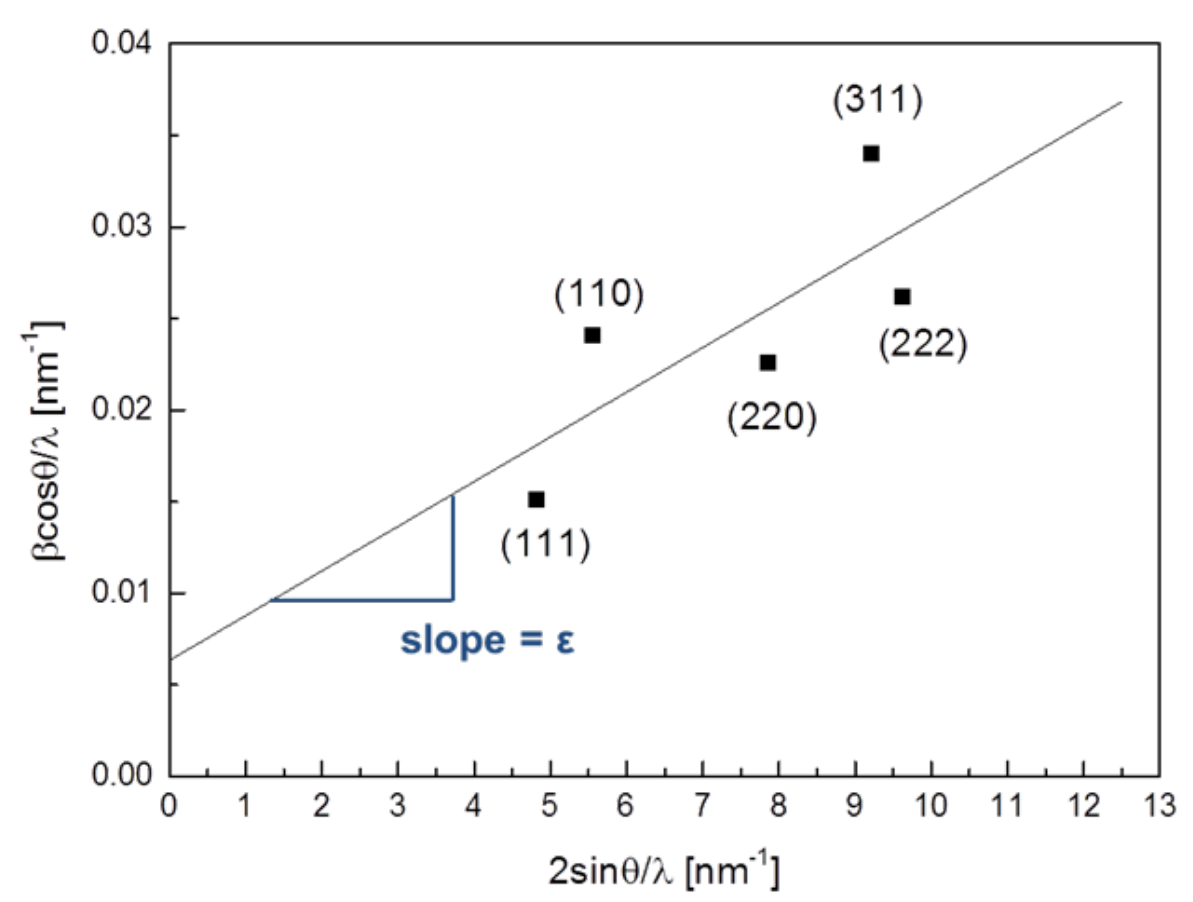

Fig. 3.22 WH plot of W12. Strain component $\varepsilon$ can be obtained from the slope.

Then, the dislocation density $\rho$ is derived as follows [113]:

$$
\rho=\frac{3}{\pi}\left(\frac{\varepsilon}{b}\right)^{2} \times 10^{18} \quad\left(1 / \mathrm{m}^{2}\right)
$$

where, $b$ is the magnitude of the Burgers vector. The magnitude of the Burgers vector is represented as follows

$$
\|b\|=\frac{a}{2} \sqrt{h^{2}+k^{2}+l^{2}}
$$

where $a$ is the lattice parameter of austenitic stainless steel, $h, k$, and $l$ are the three Miller indices of a slip direction in the crystal. In this study, the following values were used: Miller indices of a slip direction for fcc metals: $<110>$, the lattice parameter of austenitic stainless steels determined experimentally: $0.359 \mathrm{~nm}$. Thus, $b=0.254 \mathrm{~nm}$ is obtained as the Burgers vector in austenite stainless steels by Eq. (3-4) 


\subsubsection{XRD - Sample preparation}

For dislocation density measurements by XRD, mechanical grinding was done to remove at least to $70 \mu \mathrm{m}$ from surface and polishing was performed manually to $1000 \#$ on silicon carbide paper in order to remove the influence of surface a'-martensite which had formed during sample cutting. Thereafter, the surfaces were electropolished using LectroPol- 5 equipment (Struers A/S, Denmark) and A3 electrolyte (Struers A/S, Denmark) at $35 \mathrm{~V}$ for $25 \mathrm{~s}$ [114] with the help of K. Born. 


\section{Results}

\subsection{Selection of furnace}

As described in section 3.2.2.1, three types of vacuum furnaces were used in this study. In this section, we will discuss; (1) the difference of oxide layer formed by three types of furnaces, (2) the influence of the annealing time on the oxide layer thickness formed by inductive furnace, and (3) the influence of the chemical composition of stainless steels on the oxide layers. Based on these observations, selection criteria for heat treatment furnaces will be consequently given, in view of developing HE-resistant stainless steels.

\subsubsection{Oxide layer formed by three types of furnace}

As described in section 3.2.2.1, three types of vacuum furnaces, (a) inductive heater, (b) molybdenum heater, and (c) graphite heater, were used for the solution annealing process. It is well known that the thickening of the oxide layer can also be predicted by the oxide color. For stainless steels, those color changes occur in the order: yellow - brown - blue - gray - yellow (second order) with increasing oxide layer thickness [115]. The CCD camera images of three samples after SIMS analysis are shown in Fig. 4.1 (a) - (c). The square at the center of each image is the sputter crater formed by the sputter ion beam.
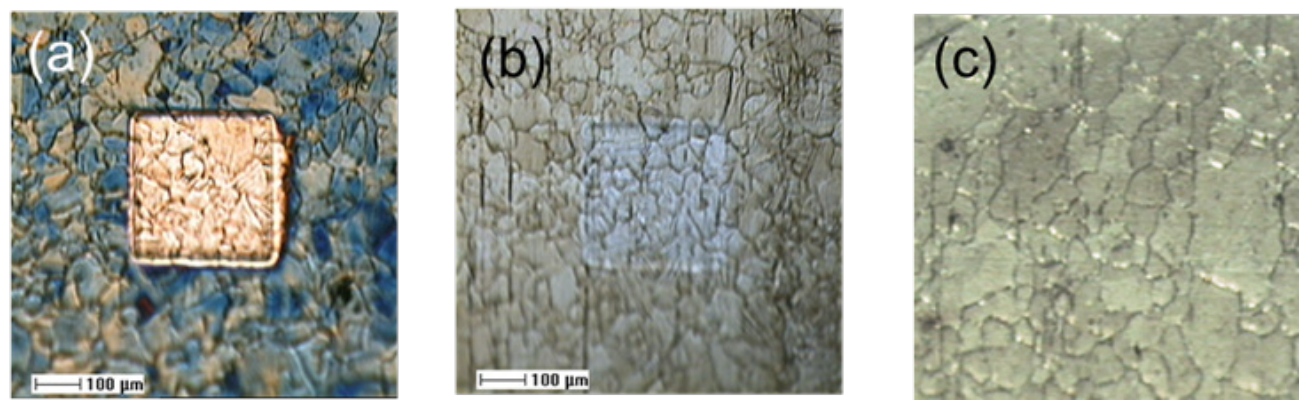

Fig. 4.1 CCD camera image of W11 annealed by (a) inductive heater, (b) molybdenum heater and (c) graphite heater. The oxide layer formed in inductive heater is predicted. A discoloration is found in (a) and (b) due to the formation of thick oxide. The picture of (c) graphite heater was taken before measurement. 
A bluish and a yellowish discoloration are found in Fig. 4.1 (a) and (b), respectively. Thus, we expect that the thickest oxide layer was formed in the inductive heater furnace.

Fig. 4.2 shows the effect of the furnace type on the oxide thickness of W11 solution annealed for $15 \mathrm{~min}$. The interface between the oxide layer and bulk was determined from the SIMS depth profiles using the point where the $\mathrm{FeO}$ signal, which is the main oxide phase, decayed to $50 \%$ of its steady state value in the oxide layer. The interface is marked with a red dotted line in Fig. 4.2. The SIMS measurement supports the results of Fig. 4.1. The oxide layer is thickest for the inductive heater furnace. The oxide layer formed in the graphite heater is significantly thinner than the others, as indicated in the inset of Fig. 4.2 (c). The vertical red dotted line in the profiles indicates the interface between oxide layer and bulk. The base pressure of each furnace is also indicated.

A layer stacking of $\mathrm{FeO}$ and $\mathrm{CrO}$ is found in all of the samples. The oxide layer thickness on the sample was $160 \mathrm{~nm}$ for the inductive heater, $35 \mathrm{~nm}$ for molybdenum heater and only $4 \mathrm{~nm}$ for graphite heater, respectively. The oxidation behavior of the samples in the three furnaces is clearly different. The thinnest oxide layer was formed in the graphite heater, although its base pressure was the highest.

This might seem to be a contradiction. However, this is explained by considering the difference of the oxygen partial pressure in the furnace, since the oxide layer thickness strongly depends on the partial pressure of oxygen in the furnace. In the graphite furnace, carbon (graphite) and the residual oxygen react to form $\mathrm{CO}$, which is known as a reducing gas, during solution annealing. This reaction reduces the partial pressure of oxygen and the surrounding atmosphere becomes a reducing character. Further, as shown in the Ellingham diagram (Fig. 2.16), the carbon oxidation $\left(2 \mathrm{C}+\mathrm{O}_{2}=2 \mathrm{CO}\right)$ line is downward-sloping. At $1050{ }^{\circ} \mathrm{C}$, the carbon oxidation line goes below the iron oxidation $\left(2 \mathrm{Fe}+\mathrm{O}_{2}=2 \mathrm{FeO}\right)$ line, the carbon can then reduce the metals presented above the carbon oxidation line. These reactions are not expected to occur in the other two furnaces with molybdenum or inductive heater, in 

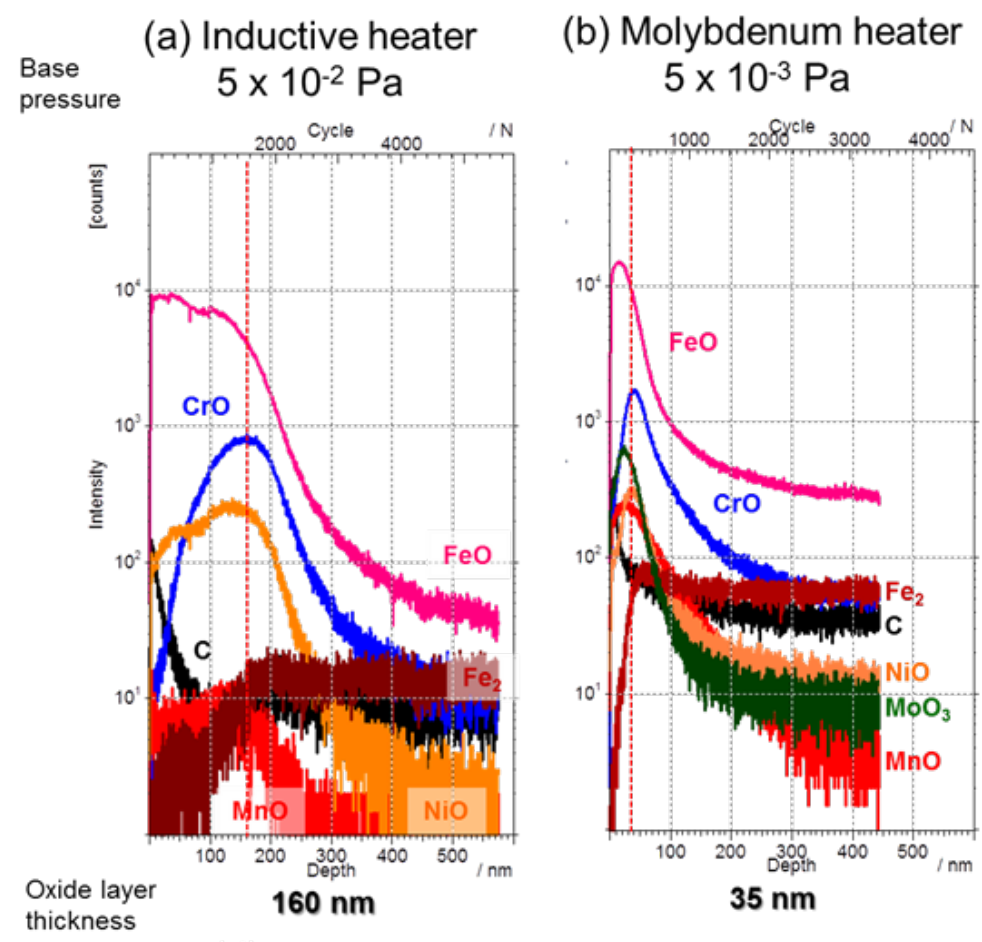

(c) Graphite heater
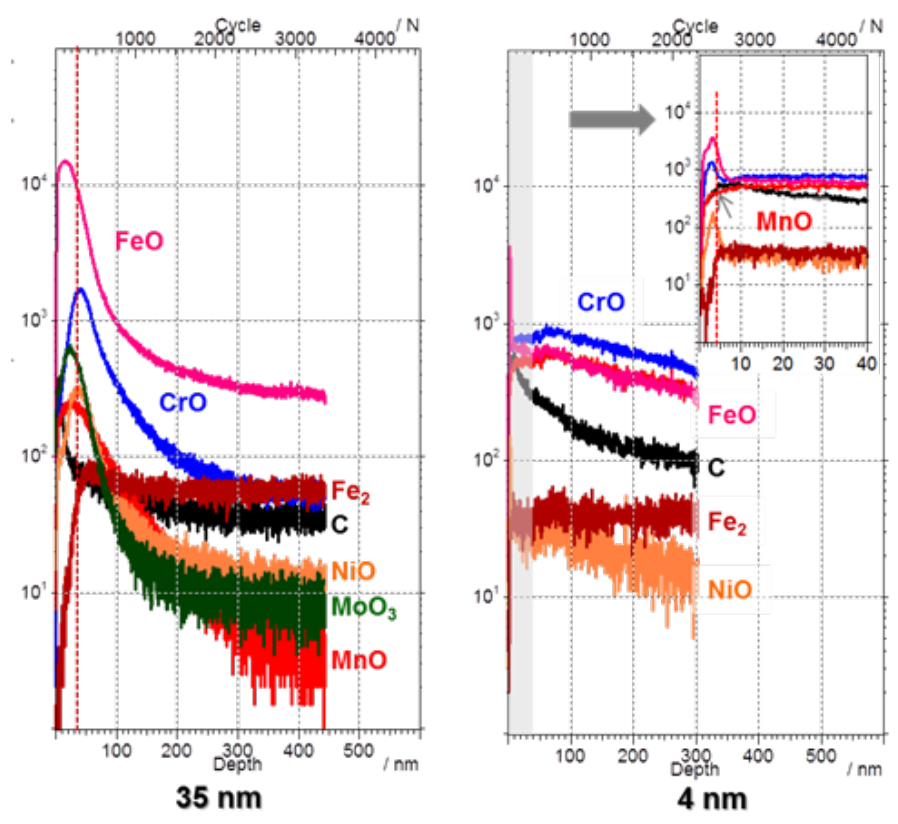

Fig. 4.2 Depth profiles on W11 solution annealed for $15 \mathrm{~min}$ by the furnace equipped with (a) inductive heater, (b) molybdenum heater, and (c) graphite heater. The interface between the oxide layer and bulk is marked with a red dotted line. A layer stacking of $\mathrm{FeO}$ and $\mathrm{CrO}$ is found on all samples. The oxide layer thickness strongly depends the partial pressure of oxygen rather than the vacuum pressure in the furnaces.

which the atmosphere is oxidizing. Hence, the difference of the oxide layer thickness detected can be ascribed to the atmosphere which was reducing or oxidizing during the heat treatment.

In the sample heated by the furnace equipped with Molybdenum heater, $\mathrm{MoO}_{3}$ is found in the oxide layer despite of only $0.3 \mathrm{wt} \%$ Mo contained in this W11 sample. A possible explanation for this $\mathrm{MoO}_{3}$ is a contamination from the furnace. Mo is often used as a high temperature material because of its high melting point $\left(2620^{\circ} \mathrm{C}\right)$. However, its resistance to oxidation is poor. When molybdenum oxides $\left(\mathrm{MoO}_{2}\right.$ and $\left.\mathrm{MoO}_{3}\right)$ form, volatilization of e.g. molybdenum trioxide occurs at $475{ }^{\circ} \mathrm{C}$ under vacuum conditions [116]. Thus, most probably the molybdenum trioxide covered the sample surface during the solution annealing process. 


\subsubsection{Influence of annealing time - Inductive heater}

The oxide layer thickness is not only a function of temperature and partial pressure of oxygen, but also a function of heating time (section 2.3.4). Fig. 4.3 shows the depth profile results on W11 annealed (a) for 15 min, (b) for $30 \mathrm{~min}$ and (c) for $60 \mathrm{~min}$ in the inductive heater furnace. A red dotted line indicates the interface between the oxide layer and bulk, as defined in section 4.1.1.
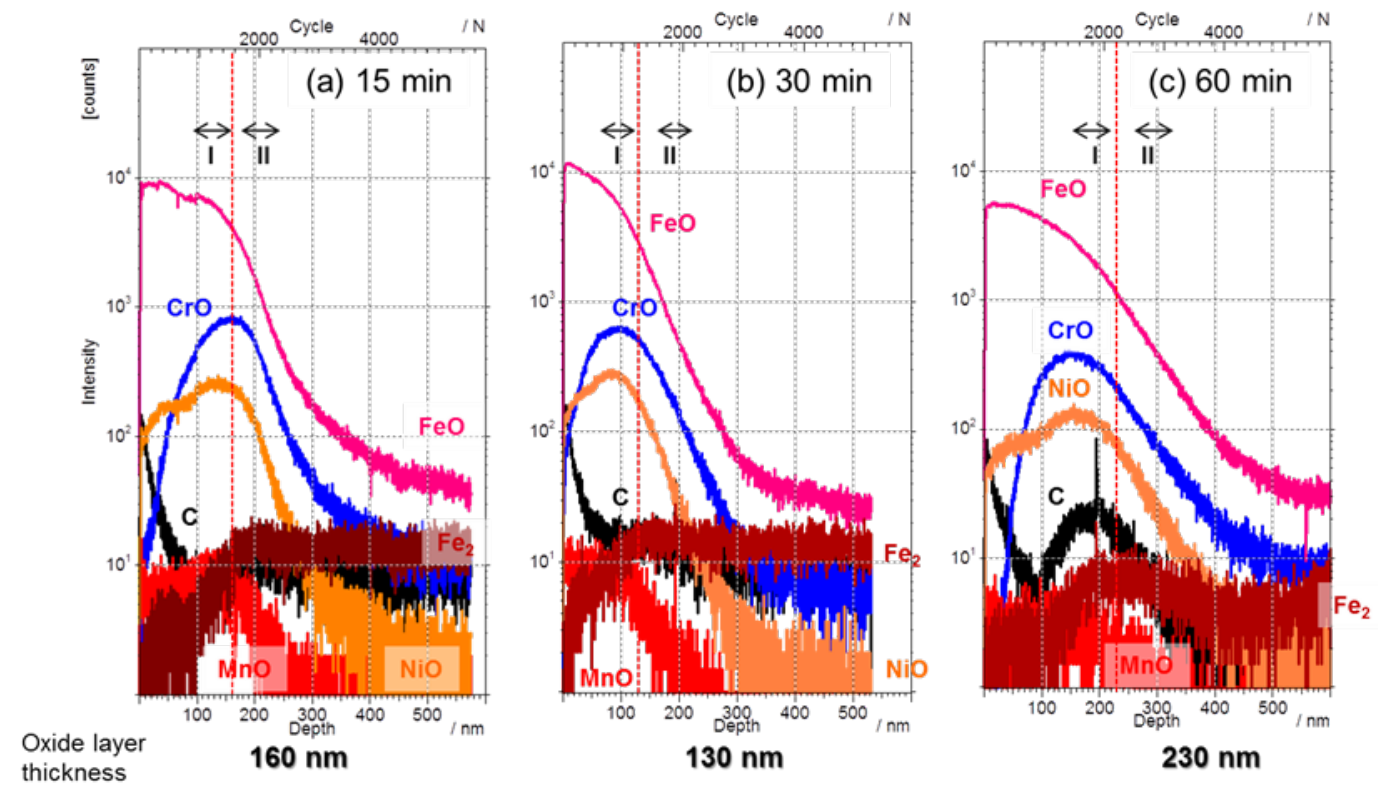

Fig. 4.3 Depth profiles in W11 solution annealed (a) for $15 \mathrm{~min}$, (b) for $30 \mathrm{~min}$, and (c) for 60 min by inductive heater furncace. The interface between the oxide layer and bulk is marked with a red dotted line. The difference in oxide layer thickness between W11 annealed for $15 \mathrm{~min}$ and for $30 \mathrm{~min}$ is small

The oxide layer thickness increases from 130 to $230 \mathrm{~nm}$, as the annealing time increases for $30 \mathrm{~min}$ to $60 \mathrm{~min}$. However, between $15 \mathrm{~min}$ and $30 \mathrm{~min}$, the change in its thickness is small, suggesting 15 min-difference has a minor impact on the growth rate of the oxide layer.

The secondary ion images of $\mathrm{FeO}, \mathrm{CrO}, \mathrm{NiO}, \mathrm{MnO}, \mathrm{Si}$ and $\mathrm{C}$ on $\mathrm{W} 11$ annealed for $15 \mathrm{~min}$, for $30 \mathrm{~min}$ and for $60 \mathrm{~min}$ are shown in Fig. 4.4 and Fig. 4.5. These images were reconstructed from two different characteristic sample depths of (I) oxide layer region and (II) beneath the interface, as indicated by the arrows in Fig. 4.3. 
Field of view: $150 \times 150 \mu \mathrm{m}^{2} \quad$ Colour scale, Each image is normalised to the brightest pixel

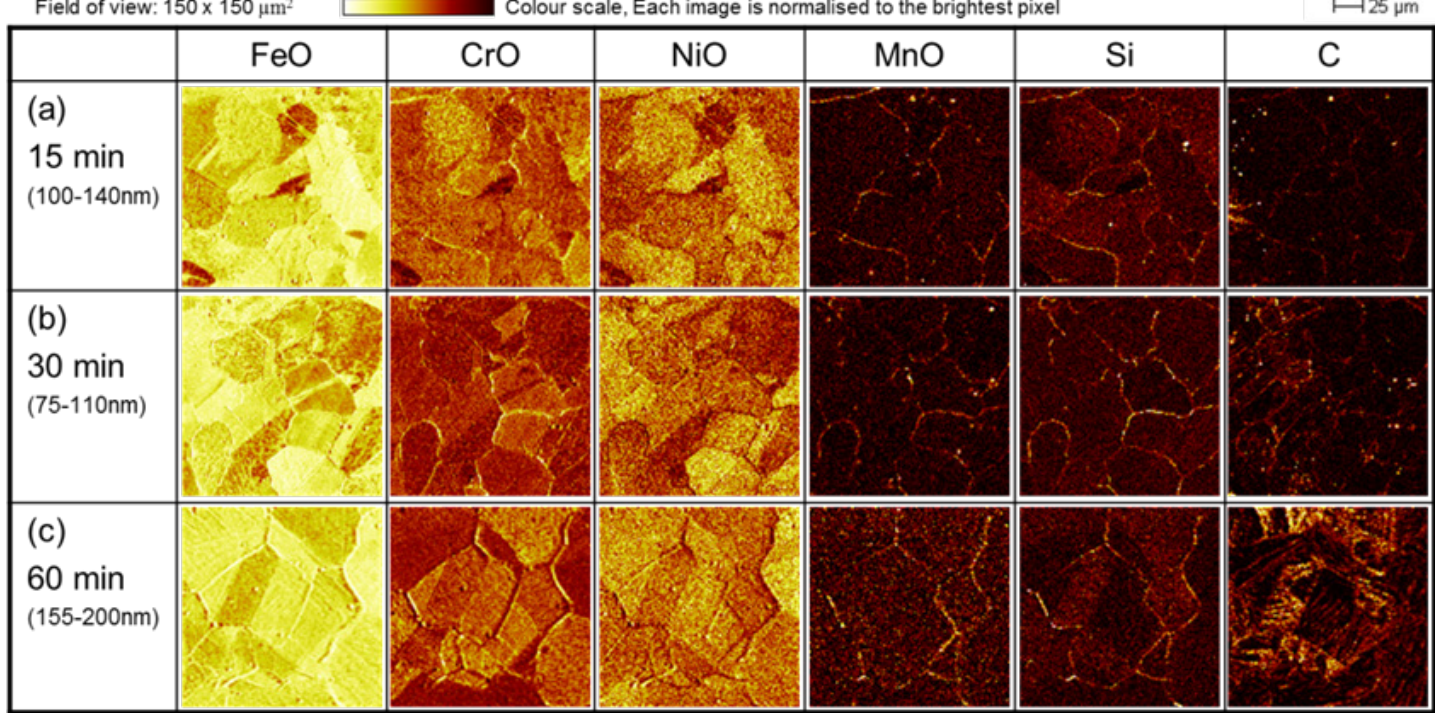

Fig. 4.4 Secondary ion images of W11 annealed by inductive heater (a) for 15 $\mathrm{min}$ (b) for $30 \mathrm{~min}$ and (c) for $60 \mathrm{~min}$ at oxide layer region (I) as indicated in Fig. 4.3. $\mathrm{FeO}, \mathrm{NiO}$ and $\mathrm{CrO}$ distribute homogeneously. $\mathrm{Si}$ and $\mathrm{MnO}$ segregate at grain boundaries.

Field of view: $150 \times 150 \mu \mathrm{m}^{2} \quad$ Colour scale, Each image is normalised to the brightest pixel

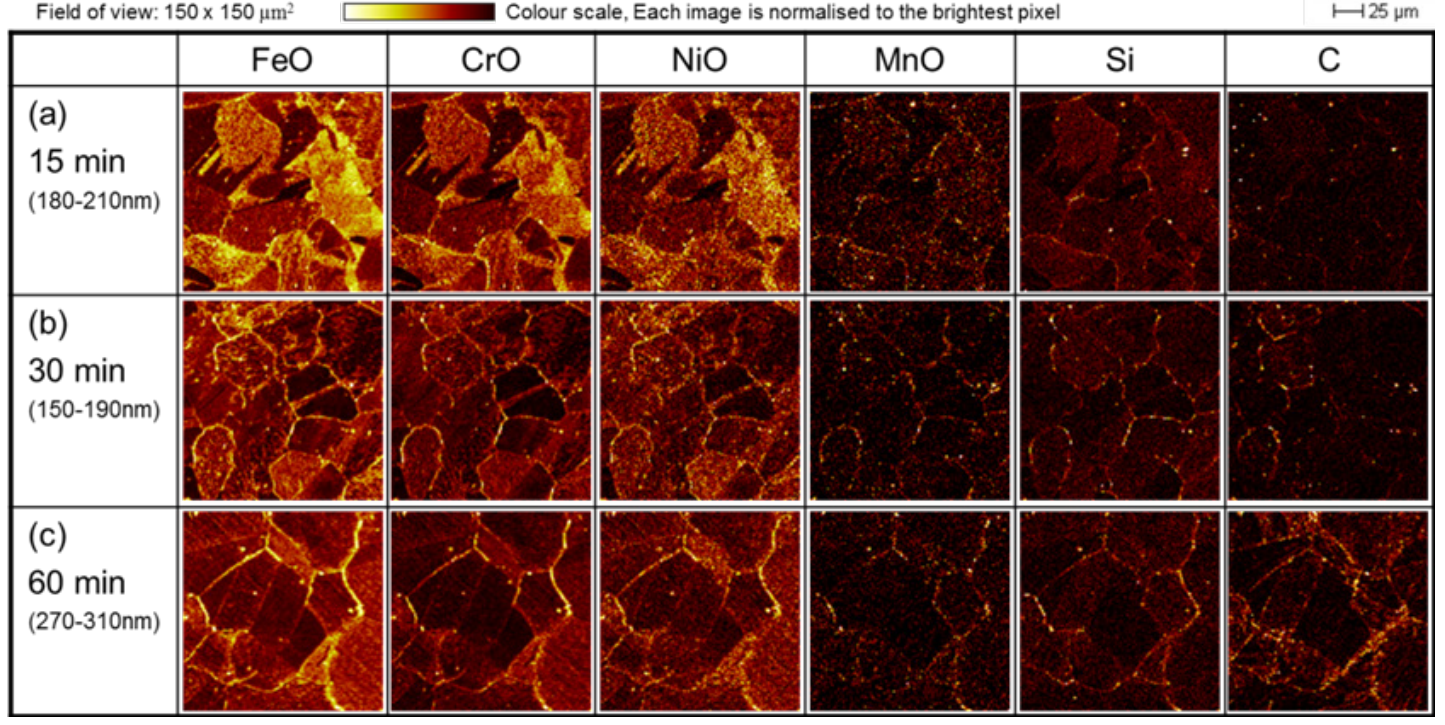

Fig. 4.5 Secondary ion images of W11 annealed by inductive heater (a) for $15 \mathrm{~min}$ (b) for $30 \mathrm{~min}$ and (c) for 60 min at beneath the interface (region II) as indicated in Fig. 4.3. The enrichment of the selected elements, except for carbon, along grain boundaries becomes visible with increasing annealing time

In the region (I), all of the samples show the same tendency that FeO, $\mathrm{NiO}$ and $\mathrm{CrO}$ distribute homogeneously, while $\mathrm{Si}$ and $\mathrm{MnO}$ segregate at grain boundaries as shown in Fig. 4.4.

In Fig. 4.5 (region II), the enrichment of the selected elements, except for carbon, along grain boundaries becomes visible with increasing annealing time. A relatively thick oxide layer was formed on W11, when annealed for 15 
min. There is no clear change in the oxide layer thickness between $15 \mathrm{~min}$ and 30 min-annealing time, as shown in Fig. 4.3.

These observations suggest that the oxidation reaction rate decreases above 15 min annealing time. This can be attributed to the growth rate of the oxide layer on stainless steels which follows either a logarithmic or an inverse rate law (compare section 2.3.4). The rate of oxidation is controlled by either anion or cation movement through the metaloxide interface [117].

At high temperatures, the grain boundary diffusion coefficients for metal ion and oxygen ion are several orders of magnitude larger than the lattice diffusion coefficient, as shown in Fig. 2.19 [94,118]. Once a thick oxide layer formed on the surface, the lattice diffusion of ions is limited. Thus, the selective oxidation at grain boundaries may dominate the reaction by the grain boundary diffusion of ions.

However, the contribution of grain boundary diffusion to the oxide layer growth rate can be smaller than that of bulk diffusion because of the small size ratio of grain boundaries to grains. Sabioni et al. studied the oxygen and chromium ion diffusion in oxide films formed on AISI 304 stainless steels which have the similar chemical composition to W11 in this study. They reported that the diffusion coefficient of oxygen ions is one order of magnitude larger than that of chromium ions at $850{ }^{\circ} \mathrm{C}[118,119]$. This suggests that oxygen ion inward diffusion is the main ion transport way for the growth mechanism of oxide films on W11.

\subsubsection{Influence of alloying elements - Mo heater}

Fig. 4.6 shows the SIMS depth profiles of (a) W11 (b) W12 and (c) W20 annealed for $15 \mathrm{~min}$ in the furnace equipped with a molybdenum heater.

An oxide layer thickness of $35 \mathrm{~nm}$ for W11, $30 \mathrm{~nm}$ for W12 and $20 \mathrm{~nm}$ for W20, respectively, was found. The oxide layer thickness defined in section 4.1.1 is marked with a red dashed line in the figures. As described in section 4.1.1, MoO in W11 could be considered as a result of contamination from the furnace itself because this sample contains only $0.3 \mathrm{wt} \%$ of molybdenum. However, the MoO profile in case of W12 and W20 shows a rather high value 

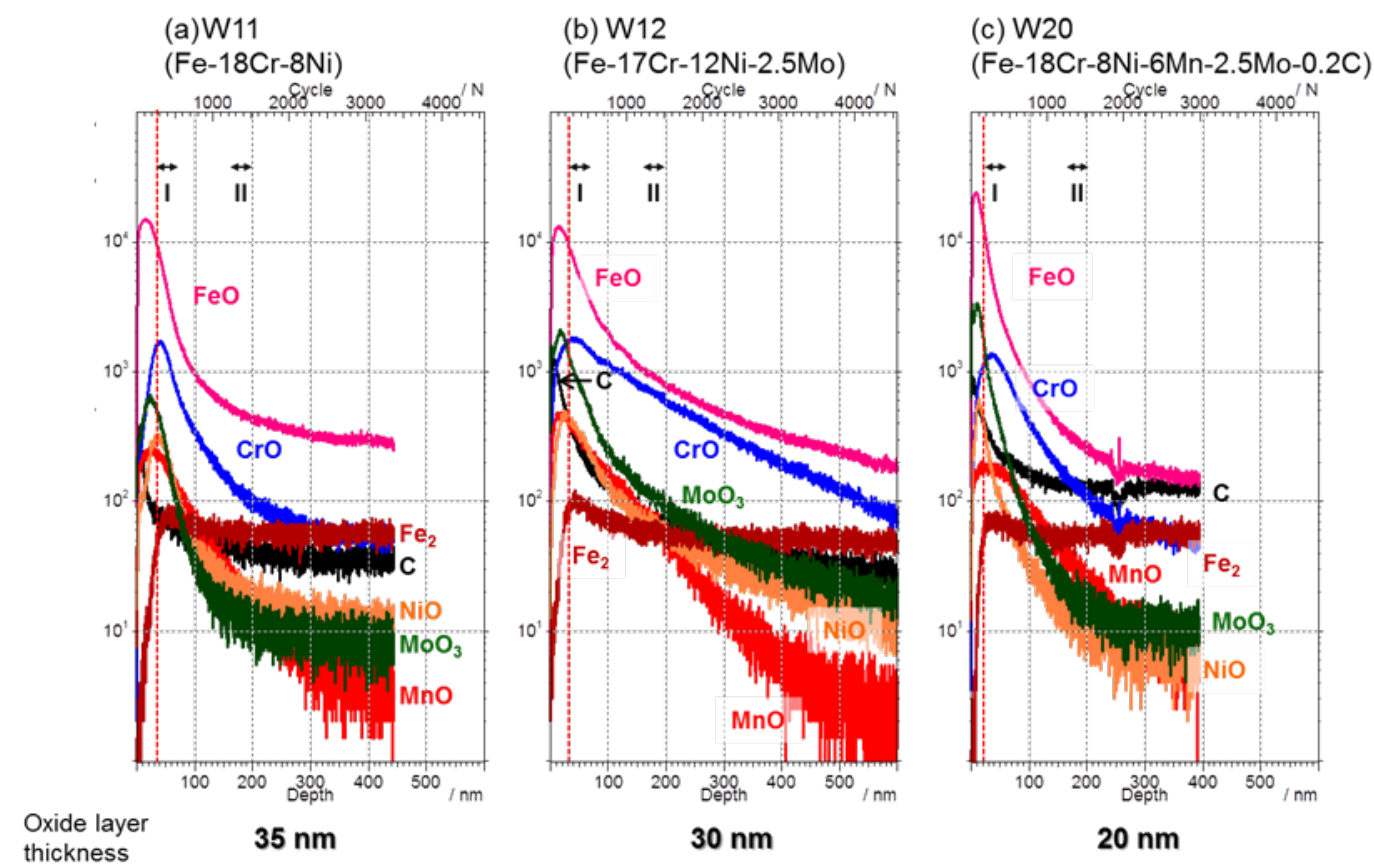

Molybdenum heater : $5 \times 10^{-3} \mathrm{~Pa}$

Fig. 4.6 Depth profiles in (a) W11, (b) W12, and (c) W20 solution annealed for 15 min by molybdenum heater furncace. A red dotted line indicates the interface between the oxide layer and bulk The intensity of selected elements in W12 decays gradually towards bulk region and shows long tails.

and contamination alone cannot explain this effect. Therefore, it is reasonable to consider that this high $\mathrm{MoO}$ intensity originates from the bulk region due to the diffusion process during annealing (note that these W12 and W20 samples originally contain $2.5 \mathrm{wt} \% \mathrm{Mo}$ ).

Further, the maximum intensity of $\mathrm{MoO}$ appears in the shallow surface region compared to that of $\mathrm{CrO}$ and $\mathrm{NiO}$. The difference in its thickness among those three samples is small, ranging around 20-35 $\mathrm{nm}$. A remarkable feature found in the profiles of W12 is that the intensities of the oxides decay rather gradually even after the oxide layer and show long tails towards the bulk region. On the contrary, the profiles in W11 and W20 show quite sharp interface and, the intensities minimize at around $300 \mathrm{~nm}$ in depth and keep a constant level thereafter.

Fig. 4.7 and Fig. 4.8 show the secondary ion images on (a) W11, (b) W12 and (c) W20 solution annealed for $15 \mathrm{~min}$ in molybdenum furnace. These images were reconstructed from two different characteristic sample depths of 
(I) just beneath the interface and (II) the underlying metallic region, as indicated by the arrows in Fig. 4.6.

\begin{tabular}{l} 
Field of view: $150 \times 150 \mu \mathrm{m}^{2}$ \\
\hline
\end{tabular}

Fig. 4.7 Secondary ion images of (a) W11, (b) W12, and (c) W20 solution annealed for 15 min by molybdenum heater furncace, reconstructed from the data taken at beneath the interface region as indicated in Fig. 4.6. The selected elements except for Si distribute homogeneously in W11 and W20. In W12, CrO and MnO highly concentrate in some grains.

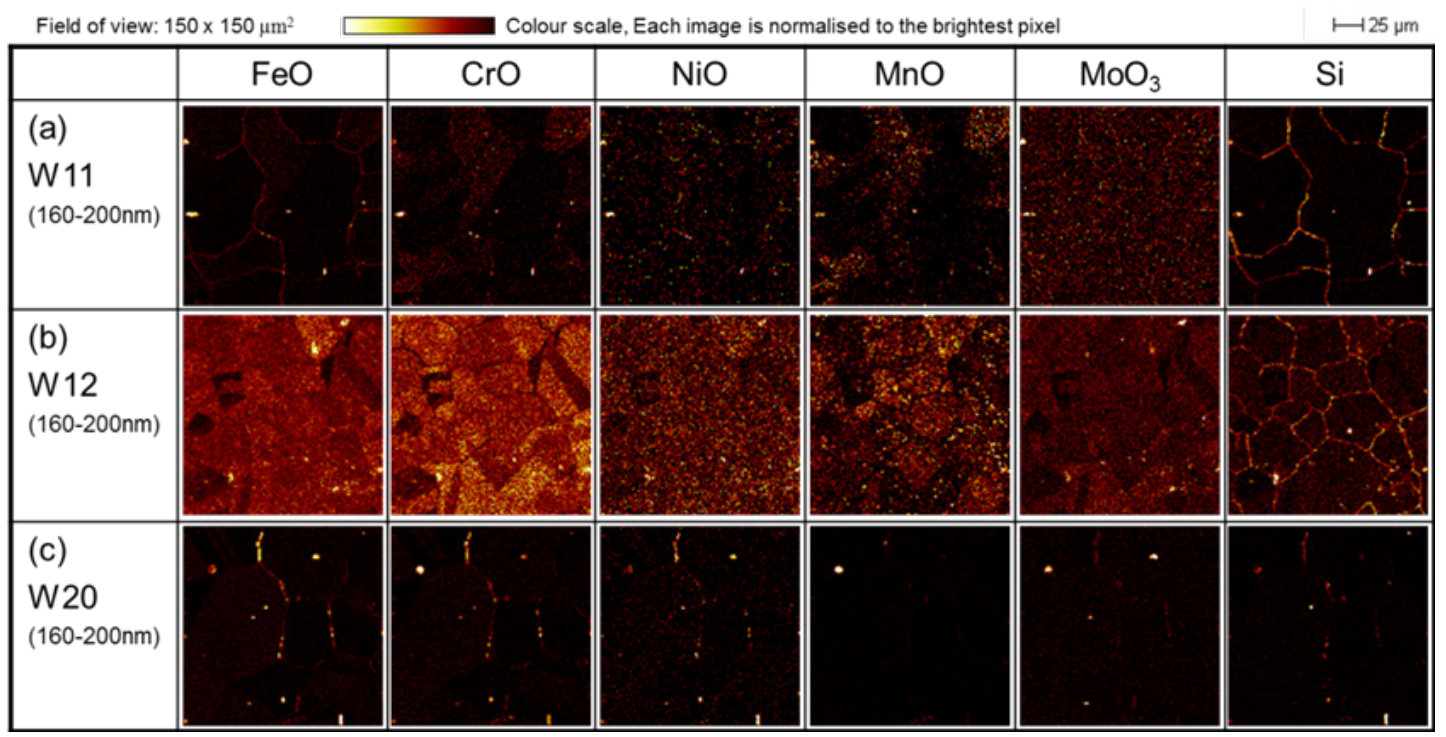

Fig. 4.8 Secondary ion images of (a) W11, (b) W12, and (c) W20 solution annealed for $15 \mathrm{~min}$ by molybdenum heater furncace at the under lying metal region as indicated in Fig. 4.6. Note that the selected elements except for Si appear still bright within the grains of W12. 
An enrichment of Si at grain boundaries is found in all three samples in Fig. 4.7. The selected oxides are more or less homogeneous in W11 and W20. In $\mathrm{W} 12, \mathrm{CrO}$ and $\mathrm{MnO}$ are localized in the grain interiors. After departing the interface region (Fig. 4.8), the signals of selected elements in W12 are still bright and distribute homogeneously, while these are only partially visible in W11 and W20. As shown in Fig. 4.6 (b), a oxide signals show gradual decay with the depth (see profile of $\mathrm{CrO}$ ). Such decaying signal is sometimes due to local enrichment of elements. However, the image in Fig. 4.8 (b) show rather homogeneous lateral distribution of oxides. This result strongly suggests that elemental localizations cannot be responsible for the decay of oxides observed in the depth profile of W12 (Fig. 4.6 (b)).

\subsubsection{Selection of furnace}

As shown above in the section 4.1.1, the oxide layer thickness strongly depends on the furnace type. A relatively thin oxide is formed in the graphite furnace while a thick oxide is formed in the Mo-furnace and the inductive heater. This difference relates to the reductive gas atmosphere in the graphite furnace, where oxygen and carbon are consumed together to form carbon oxides. The Formation of thick oxide layers leads to chemical composition gradients in the near surface region. To avoid this, the graphite furnace was mainly used in the following studies. Just for the study on the influence of the a'-martensite on HE (section 4.2.1), the Mo-furnace was used. These results were obtained at the early stages of this study.

\subsection{Reference steel: Tensile test at RT}

As described in chapter 1 , many aspects affect the mechanical properties of alloys in hydrogen atmosphere. In this section, we focus on the reference steel (W11) prepared at different conditions. The microstructural effects arising from processing and heat treatment will be shown. It should be noted that the tensile tests shown in this section were performed at room temperature. The results obtained on samples tested at lower temperature 
will be shown in section 4.3 .

\subsubsection{Influence of $a^{\prime}$-martensite}

Both strain-induced a'-martensite and the oxide layer are considered as major factors influencing RRA and thus the HE susceptibility. Strain-induced a'-martensite acts as hydrogen diffusion path, whereas the oxide layer prevent hydrogen entry in metal.

This section focuses on the results of tensile tests and SIMS in order to consider the impact of the strain-induced a'-martensite and the oxide layer separately. First, the results on W11 with and without a'-martensite are presented. One of the W11 samples was in machined condition, that is, the austenite phase $(\mathrm{Y})$ partially transformed to a'-martensite in the surface region. An a'-martensite free W11 sample was produced by solution annealing. The solution annealing was performed at $1050{ }^{\circ} \mathrm{C}$ for $15 \mathrm{~min}$ by either molybdenum or graphite furnace after the machining.

\subsubsection{Tensile test results}

Microstructural observations were also carried out by Martín [16] using optical microscope to confirm surface a'-martensite. Fig. 4.9 shows the cross section of W11 (a) with a'-martensite and (b) without a'-martensite.
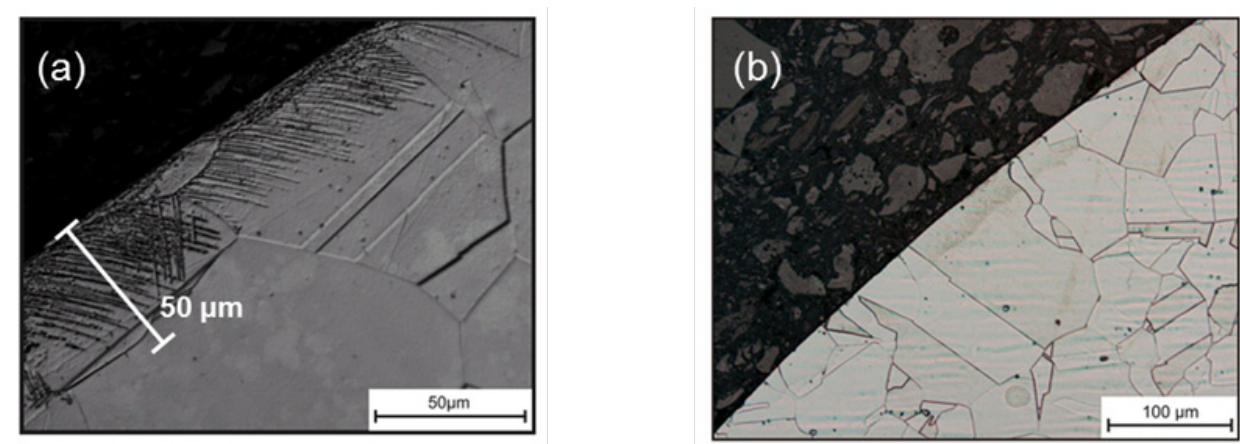

Fig. 4.9 Cross section of W11 (a) with $\alpha^{\prime}$-martensite and (b) without $\alpha^{\prime}$-martensite [16]. The strain-induced $\alpha^{\prime}$-martensite $50 \mu \mathrm{m}$ in depth below surface resulting from the machining process can be observed on W11 with $\alpha^{\prime}$-martensite. (Image from Martín [16], with permission of the author) 
The strain-induced a'-martensite, which formed during the machining process, is observed in the surface region with a thickness of around $50 \mu \mathrm{m}$ (Fig. 4.9 (a)). a'-martensite free surface is obtained by solution annealing at $1050{ }^{\circ} \mathrm{C}$ for 15 min after the machining process, as confirmed by Fig. 4.9 (b).

Fig. 4.10 shows the different fracture surfaces of W11 after the tensiles test of (a) the as machined state and (b) after subsequent solution annealing, respectively [16]. The tensile tests were performed in hydrogen at $40 \mathrm{MPa}$ at room temperature. W11 with a'-martensite exhibits the typical brittle appearance and a number of vertical secondary cracks to longitudinal gauge section (Fig. 4.10 a), whereas W11 without a'-martensite shows ductile fracture (Fig. $4.10 \mathrm{~b}$ ).

(a)

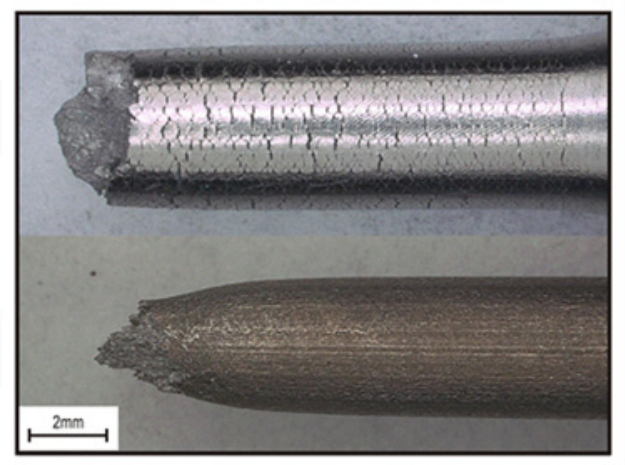

Fig. 4.10 Macroscopic view of W11 after tensile test at RT in hydrogen at 40 $\mathrm{MPa}$. (a)as machined (with $\alpha^{\prime}$-martensite) and (b) solution annealed (without $\alpha^{\prime}$-martensite) [16]. W11 with $\alpha^{\prime}$-martensite exhibits the typical brittle appearance. A number of vertical secondary cracks to longtitudinal gauge section are observed. W11 without $\alpha$ '-martensite shows ductile fracture. (Image from Martín [16], with permission of the author)

After the tensile tests, the degree of $\mathrm{HE}$ is evaluated by determining the relative reduction of area (RRA) which is the ratio of the reduction of area (RA) in hydrogen to that in air. An RRA of $100 \%$ indicates that there is no influence of hydrogen on the macroscopic tensile ductility. The RA and RRA of both samples were also reported by Martín [16] as listed in Table 4.1. 
Table 4.1 RA and RRA values of W11 with and without $\alpha$ ' martensite determined by tensile testing at room temperature in air and hydrogen atmosphere at $40 \mathrm{MPa}$ [16]. (Data from Martín [16], with permission of the author)

\begin{tabular}{|c|c|c|c|c|}
\hline Sample & Atmosphere & RA (\%) & average RA (\%) & RRA (\%) \\
\hline \multirow{4}{*}{ with $\alpha^{\prime}$ martensite } & \multirow{2}{*}{ air } & 82.1 & \multirow{2}{*}{82} & \multirow{4}{*}{51.6} \\
\hline & & 81.8 & & \\
\hline & \multirow{2}{*}{$\mathrm{H}_{2}$} & 30.7 & \multirow{2}{*}{42.3} & \\
\hline & & 53.9 & & \\
\hline \multirow{4}{*}{$\begin{array}{l}\text { without } \alpha^{\prime} \\
\text { martensite }\end{array}$} & \multirow{2}{*}{ air } & 81.4 & \multirow{2}{*}{81.7} & \multirow{4}{*}{84.1} \\
\hline & & 81.9 & & \\
\hline & \multirow{2}{*}{$\mathrm{H}_{2}$} & 70.1 & \multirow{2}{*}{68.7} & \\
\hline & & 67.2 & & \\
\hline
\end{tabular}

The RRA of W11 with a'-martensite is as low as $51.6 \%$, while it is $84.1 \%$ for W11 without a'-martensite. This proves that W11 with a'-martensite has a lower ductility than W11 without a'-martensite when tested in hydrogen gas. This result clearly demonstrates that W11 with a'-martensite is more susceptible to HE than W11 without a'-martensite.

\subsubsection{SIMS analysis}

During the machining process, not only a'-martensite but also an oxide layer is formed, instantaneously. These oxide layers formed on W11 with a'-martensite might differ from that on W11 with a'-martensite due to different surface conditions and local chemistry.

The main purpose of this section is to characterize the oxide layer formed on both samples during the machining process. No further surface polishing is allowed in order to keep the surface in its original condition. As a result, the SIMS analysis has been carried out on the samples with semi-cylindrical geometry having wavy height modulations as shown in section 3.2.1.7. Therefore, unfortunately, degradation of the depth profiles to certain extent inevitable. Nevertheless, a reasonable characterization is possible by scanning in parallel to this height modulation caused by the machining. The sample geometry, and the scanning direction are schematically shown in Fig. 4.11 
(a) Sample geometry

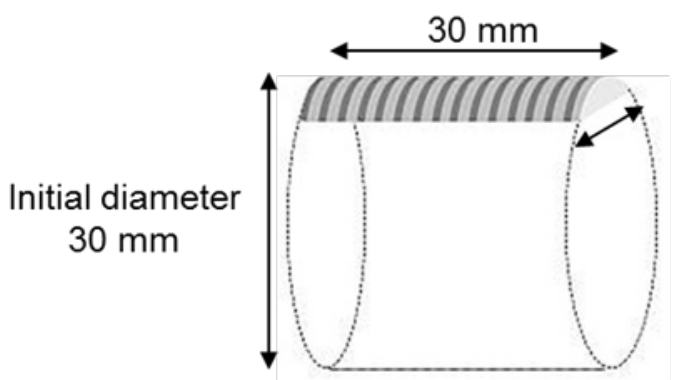

(b) Scanning direction

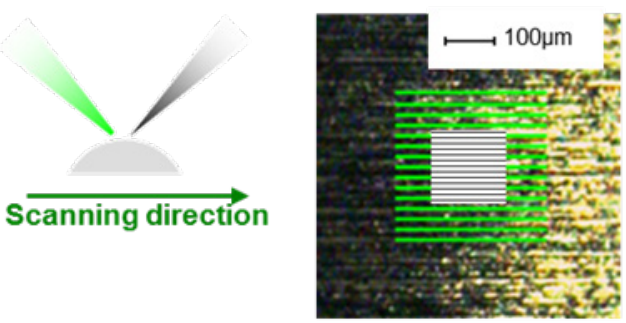

Fig. 4.11 (a) Sample geometry and (b) Scanning direction

The results of these SIMS characterizations are shown in Fig. 4.12. Here, depth profiles of the upper $25 \mathrm{~nm}$ of W11 with and without a'-martensite are shown.
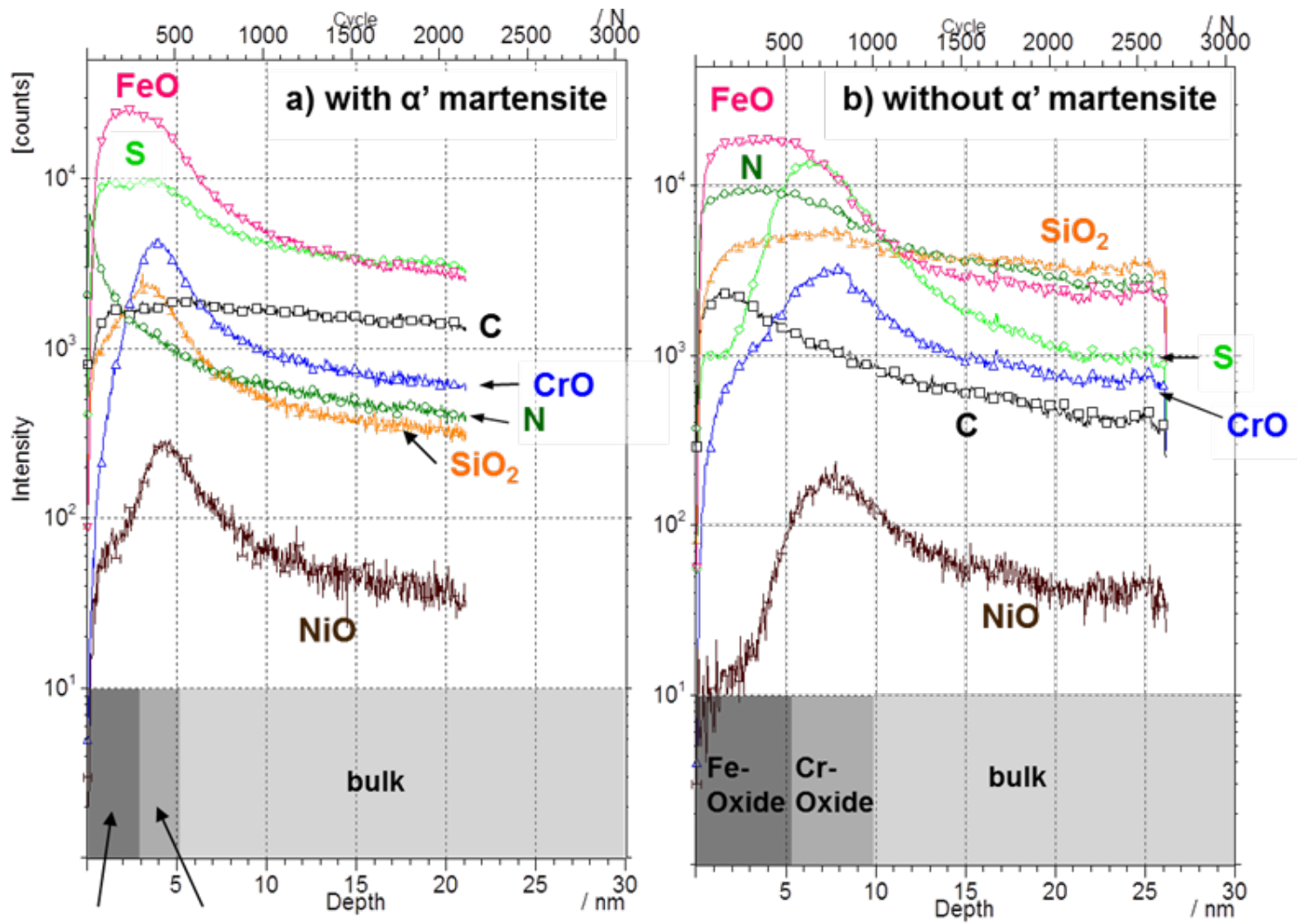

Fe-Oxide Cr-Oxide

Fig. 4.12 Depth profiling on W11 (negative polarity) (a) in as machined condition (b) solution annealed for $15 \mathrm{~min}$. The layered stacking of Fe- and $\mathrm{Cr}$-oxide with a thickness of $5 \mathrm{~nm}$ for as machined and $9 \mathrm{~nm}$ for solution annealed for $15 \mathrm{~min}$. 
The oxides with high intensities found in the beginning of each measurement shown in Fig. 4.12 (a) and (b), i.e. at the left side are stacked layers of $\mathrm{FeO}, \mathrm{CrO}, \mathrm{NiO}$ and $\mathrm{SiO}_{2}$. These oxides form the surface oxide layer. After passing the surface oxide layer, the oxides keep the relatively high intensities compared to the results on the flat samples (see Fig. 4.16). This is due to the surface modulation and curvature, as described in section 3.2.1.7. Nitrogen is detected as CNO due to its low sensitivity. The stacking sequence of the $\mathrm{FeO}$ and $\mathrm{CrO}$ signal is visible in both profiles, as indicated in the bottom of each figure. This confirms a layered stacking of two different oxides. Most probably, these oxides are $\mathrm{Fe}_{2} \mathrm{O}_{3}$ and $\mathrm{Cr}_{2} \mathrm{O}_{3}$, the most stable oxides of $\mathrm{Fe}$ and $\mathrm{Cr}$.

The main chemical species in W11 with a'-martensite changes from the top most surface as shown in Fig. 4.12 (a). First, $\mathrm{N}$ shows an increased intensity at the top most surface. A maximum $\mathrm{FeO}$ signal is detected in the first $2.5 \mathrm{~nm}$-depth. In addition, the $\mathrm{S}^{-}$signal appears also high in the same region, and $\mathrm{NiO}$ is slightly showing a low intensity shoulder at about $2 \mathrm{~nm}$ depth. The $\mathrm{SiO}_{2}{ }^{-}$signal reveals a maximum at about $3.5 \mathrm{~nm}$ depth. Then, in a depth of about $4 \mathrm{~nm}$ the $\mathrm{CrO}$ signal marks almost maximum. At a depth of 4 $\mathrm{nm}$ the $\mathrm{NiO}$ signal shows its maximum. $\mathrm{C}$ is distributed randomly, but with a high constant intensity.

For W11 without a'-martensite (Fig. 4.12 (b)), a maximum FeO signal is present over the first $6 \mathrm{~nm}$. The $\mathrm{S}^{-}$signal is shifted towards the sample interior and reaches its maximum at $7 \mathrm{~nm}$. The $\mathrm{CrO}$ intensity has its maximum at $8 \mathrm{~nm}$ depth and a shoulder at $3 \mathrm{~nm}$ depth. $\mathrm{NiO}$ intensity is low for the uppermost layers and reaches its maximum at about $8 \mathrm{~nm}$. The $\mathrm{C}^{-}$ signal is slightly increased towards the sample surface showing a maximum at $2 \mathrm{~nm}$. Furthermore, an increased concentration of $\mathrm{N}^{-}$is detected at the sample surface. This is in contrast to the $\mathrm{N}$ profile of the as machined sample, i.e. with a'-martensite, shown in Fig. 4.12 (a)

This difference in the $\mathrm{N}$ profile may be attributed to the effect of annealing. Nitrogen is present as an impurity in the Ar gas, used for quenching after the solution annealing. However, since the Ar gas contains 
less than $10 \mathrm{ppm}$ of $\mathrm{N}$, its influence is supposed to be small. Therefore, the high $\mathrm{N}$ concentration most probably arises from the bulk material itself, containing about $0.07 \mathrm{wt} \% \mathrm{~N}$.

During solution annealing in high vacuum, $\mathrm{N}$ and $\mathrm{C}$ diffuse toward the surface and accumulates. This is attributed to the high defect density at around the surface caused by martensitic transformation.

The $\mathrm{SiO}_{2}$ content is nearly constant and higher than in Fig. 4.12 (b). A difference in the chemical potential for oxygen directs Si towards the sample surface where oxygen is provided.

The lateral distributions of $\mathrm{FeO}^{-}, \mathrm{CrO}^{-}, \mathrm{NiO}^{-}, \mathrm{SiO}_{2}^{-}$and $\mathrm{C}^{-}$on $\mathrm{W} 11$ with a'-martensite (as-machined) is shown in Fig. 4.13. These images are derived from three characteristic sample depth regions: (a) the oxide layer : 0 - $6 \mathrm{~nm}$, (b) the region below the oxide layer : 8-14 nm, and (c) bulk : $45-51 \mathrm{~nm}$ (for the depth scaling see Fig. 4.12 (a)). Note that each image is normalized to the brightest pixel according to the color scale.

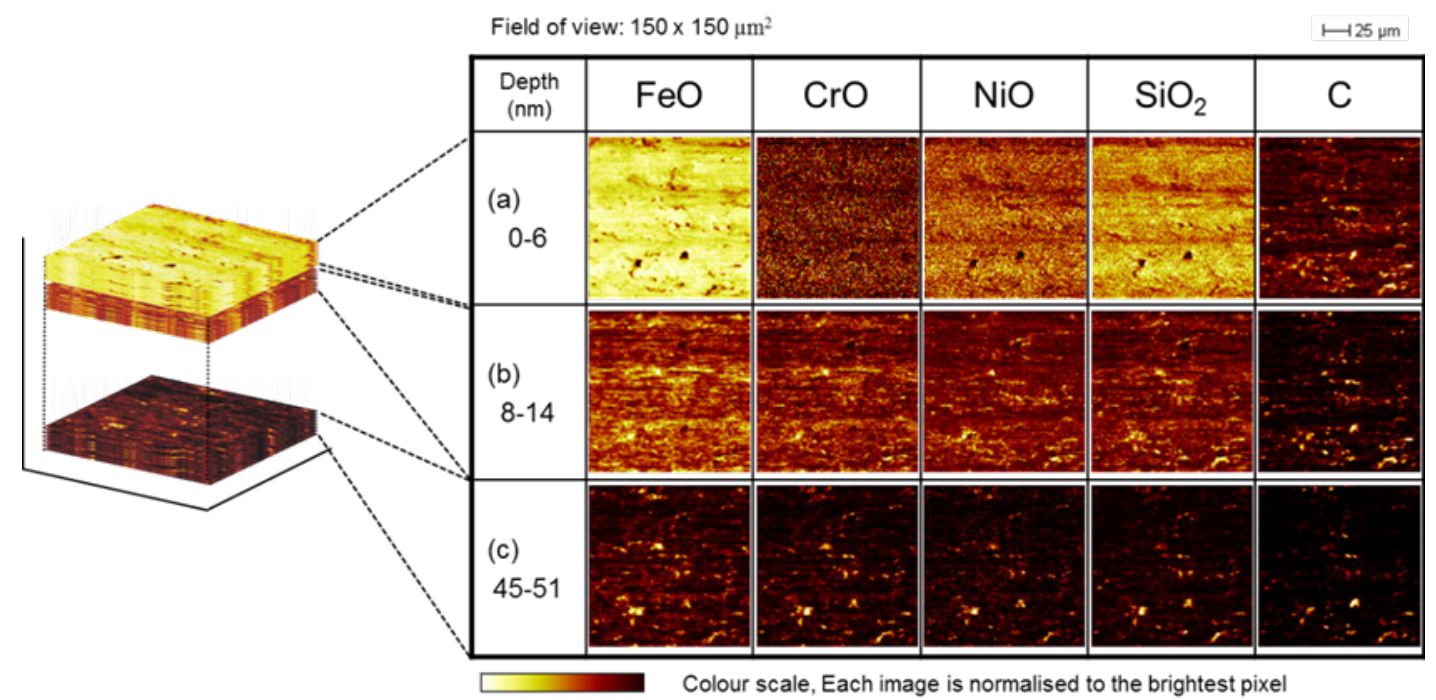

Fig. 4.13 Secondary ion images of W11 with $\alpha^{\prime}$ martensite at different sample depths. Relatively homogeneous desitribution appears.

The bright spots in (b) and (c) correlate, suggesting that the surface remained partially intact resulting from an uneven sputtering process due to strong irregularity of the initial surface. The microstructure of 
strain-induced a'-martensite, which had formed during the machining process, is smaller than the lateral resolution of the instrument. Thus, no remarkable distribution of the selected ions is observed on this microscopical scale.

The lateral elemental distributions in W11 without a'-martensite (annealed) are shown in Fig. 4.14 by using the same method as was employed for Fig. 4.13. Selected depth ranges are (a) 4 - $10 \mathrm{~nm}$ (b) $12-18 \mathrm{~nm}$ and (c) 48 - $54 \mathrm{~nm}$, according to the profiles in Fig. 4.12 (b).

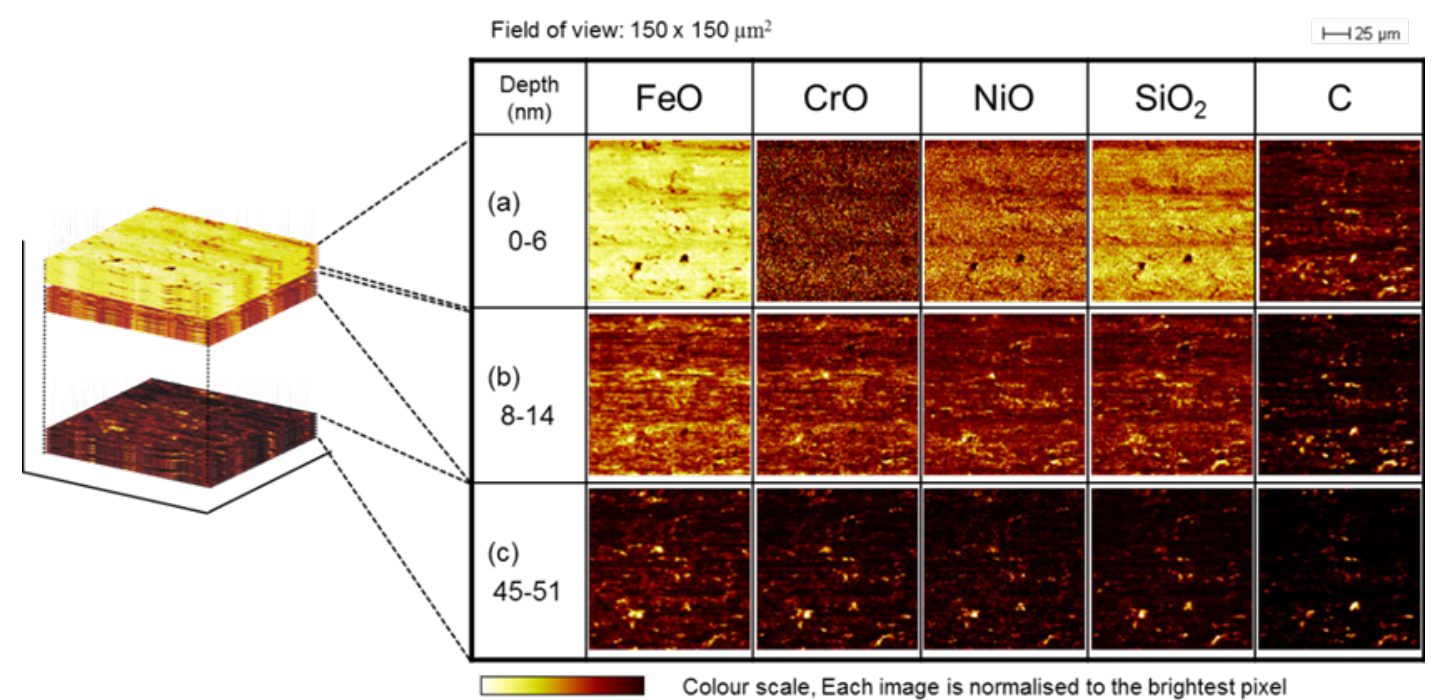

Fig. 4.14 Secondary ion images of W11 without $\alpha^{\prime}$ martensite at different sample depths. Grain sizes of about $30 \mu \mathrm{m}$ are visible. The distribution of $\mathrm{SiO}_{2}{ }^{-}$at grain boundaries suggests the $\mathrm{Si}$ diffusion from the sample interior to sample surface through grain boundaries during the annealing.

Grain sizes of about $30 \mu \mathrm{m}$ are visible in all images except for that of carbon. In a depth of $12-18 \mathrm{~nm}$ (b) and 48 - $54 \mathrm{~nm}$ (c) dots-like distributions of $\mathrm{CrO}^{-}$is sporadically observed in some grains. This may be attributed to the formation of chromium carbide, which is often considered to lead to the transformation of a'-martensite in the depletion zone of chromium and carbon [15]. However, it is evident that there is no chromium carbide since the dots-like distribution is not observed for $\mathrm{C}$ distribution, here. Hence, the formation of chromium carbides can be ignored in this study. Further, annealing at $1050{ }^{\circ} \mathrm{C}$ causes dissolution of all carbides and quenching in $\mathrm{Ar}$ 
gives a rate of cooling fast enough to hold all carbon in supersaturated solution.

As can be seen in the $\mathrm{SiO}^{-}$image in a depth of $12-18 \mathrm{~nm}$ (b) and $48-54$ $\mathrm{nm}(\mathrm{c}), \mathrm{SiO}^{-}$is located at grain boundaries. This $\mathrm{Si}$ segregation is suggested to result from the solution annealing, as Si can easily diffuse along grain boundaries (section 2.3.4.2). As a result, Si diffuses from the sample interior to sample surface through grain boundaries during the annealing treatment. The constant Si profile in Fig. 4.12 (b) also suggests this phenomenon.

\subsubsection{Influence of annealing time}

As described in chapter 1 , the reported RRA values on similar austenitic stainless steels with low-nickel content scatter significantly. This variation in the RRA-values was found in this study, too. In the following sections, the difference in RRA of reference specimens annealed for $15 \mathrm{~min}$ and for $30 \mathrm{~min}$ will be presented. The reference specimens (W11) investigated here were solution annealed at $1050{ }^{\circ} \mathrm{C}$ by graphite heater, eliminating the possible impact of the surface martensite phase. Tensile tests were performed at room temperature in air at atmospheric pressure and in hydrogen atmosphere at $40 \mathrm{MPa}$.

As shown in section 4.1.2 and in Fig. 4.3, the impact of annealing time on the oxide layer thickness was found to be small, in case of the treatment in inductive heater furnace. This section further investigates the impact of the annealing time by using the graphite heater furnace, which already demonstrated the lowest oxidation impact among all the furnaces tested in the preliminary experiments.

\subsubsection{Tensile test results}

The RA and RRA obtained on W11 annealed for $15 \mathrm{~min}$ and $30 \mathrm{~min}$ in graphite heater furnace are summarized in Table 4.2 [20].

Fig. 4.15 shows the RRA-value of W11 annealed for $15 \mathrm{~min}$ and $30 \mathrm{~min}$ plotted as a function of $\mathrm{Ni}$ content of the austenitic stainless steels including 


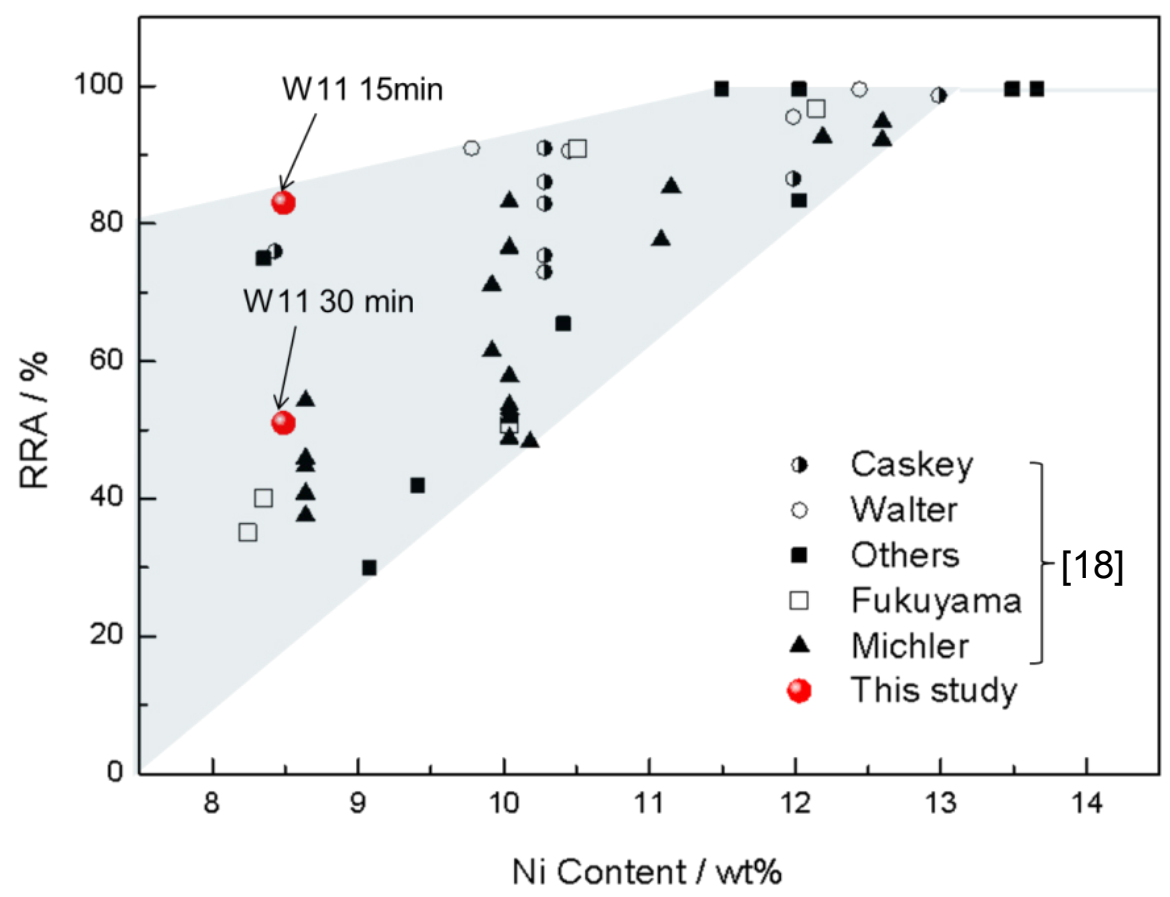

Fig. 4.15 Relative reduction of area (RRA) depending on the Ni content of the austenitic stainless steel studied here including the results of different research groups [18]. All tests were performed at room temperature. The variability in RRA values is considerably large below $10 \mathrm{wt} \% \mathrm{Ni}$ (reported in Fig. 1.3). The RRA value of W11 changes by annealing time. It is higher when annealed for 15 min than when annealed for $30 \mathrm{~min}$.

the results obtained by different research groups. All of the plots shown here are based on tests performed at room temperature.

As pointed out by Michler et al. [18], a large scatter of the RRA-values was found for the austenitic stainless steels, containing less than $10 \mathrm{wt} \% \mathrm{Ni}$. Interestingly, the sample W11 annealed for 15 min was found to be rather insusceptible against HE, while W11 annealed for $30 \mathrm{~min}$ is susceptible to HE.

The repeatability measurements were conducted at the same test conditions. The obtained values are almost identical to the results shown in Table 4.2.

The two samples have the same chemical composition and an almost identical grain size of about $50 \mu \mathrm{m}$. The only difference between these two samples is the solution annealing time. The RA obtained in air shows comparable value for both samples, implying that the difference in solution 
annealing time has an impact only on the fracture process under hydrogen atmosphere.

Table 4.2 RA and RRA values of reference stainless steels (W11) solution annealed for $15 \mathrm{~min}$ and $30 \mathrm{~min}$ by graphite furnace. Tensile tests were peformed at room temperature in air at atmospheric pressure and in hydrogen atmosphere at $40 \mathrm{MPa}$ [20]. (Data from Martín [20], with permission of the author)

\begin{tabular}{ccccc}
\hline Alloy & $\begin{array}{c}\text { Annealing time } \\
\text { (min) }\end{array}$ & air & $\mathrm{H}_{2}$ & $\mathrm{RRA}(\%)$ \\
& 15 & 82 & 69 & 84 \\
\hline W11 & 30 & 81 & 40 & 49 \\
W11 & & & & \\
\hline
\end{tabular}

\subsubsection{SIMS analysis}

SIMS analyses were performed to unravel the reason for the gap in RRA found between the two samples annealed for $15 \mathrm{~min}$ and $30 \mathrm{~min}$, respectively. Depth profile results on W11 in the as polished condition as well as annealed for $15 \mathrm{~min}$ and annealed for $30 \mathrm{~min}$ are shown in Fig. 4.16 (a), (b) and (c).

On the as polished sample, (Fig. 4.16 a) only a thin surface oxide is observed. The annealed samples (b and c) show still high intensities of $\mathrm{Fe}^{-}$, $\mathrm{Cr}^{-}, \mathrm{Ni}^{-}, \mathrm{Mn}$-oxide signals even after passing a thin surface oxide layer compared to the profile of the polished sample. The intensity of $\mathrm{Fe}^{-}, \mathrm{Cr}^{-}, \mathrm{Ni}^{-}$ and Mn-oxide remains constant for at least $80 \mathrm{~nm}$ in W11 annealed for 15 min (Fig. 4.16 b). The intensity of $\mathrm{Fe}^{-}, \mathrm{Cr}^{-}, \mathrm{Ni}^{-}$and $\mathrm{Mn}^{-}$oxide signal in W11 annealed for 30 min decreases gradually with increasing depth (Fig. 4.16 c). In the following, the former type of oxide is named as "high constant oxide level" and the latter type of oxide as "decaying sub-surface oxide". The high constant oxide level was also found on W11 annealed for $15 \mathrm{~min}$ in Mo furnace, as shown in Fig. 4.6, although their surface oxide thickness is relatively thick compared with that of W11 annealed in graphite heater. A layer stacking is depicted with the scheme of a layer model in Fig. 4.16. The outer layer is a $\mathrm{Fe}$-rich oxide while the inner layer is a mixed $\mathrm{Fe}-\mathrm{Cr}-\mathrm{Ni}-\mathrm{Mn}$ oxide. 


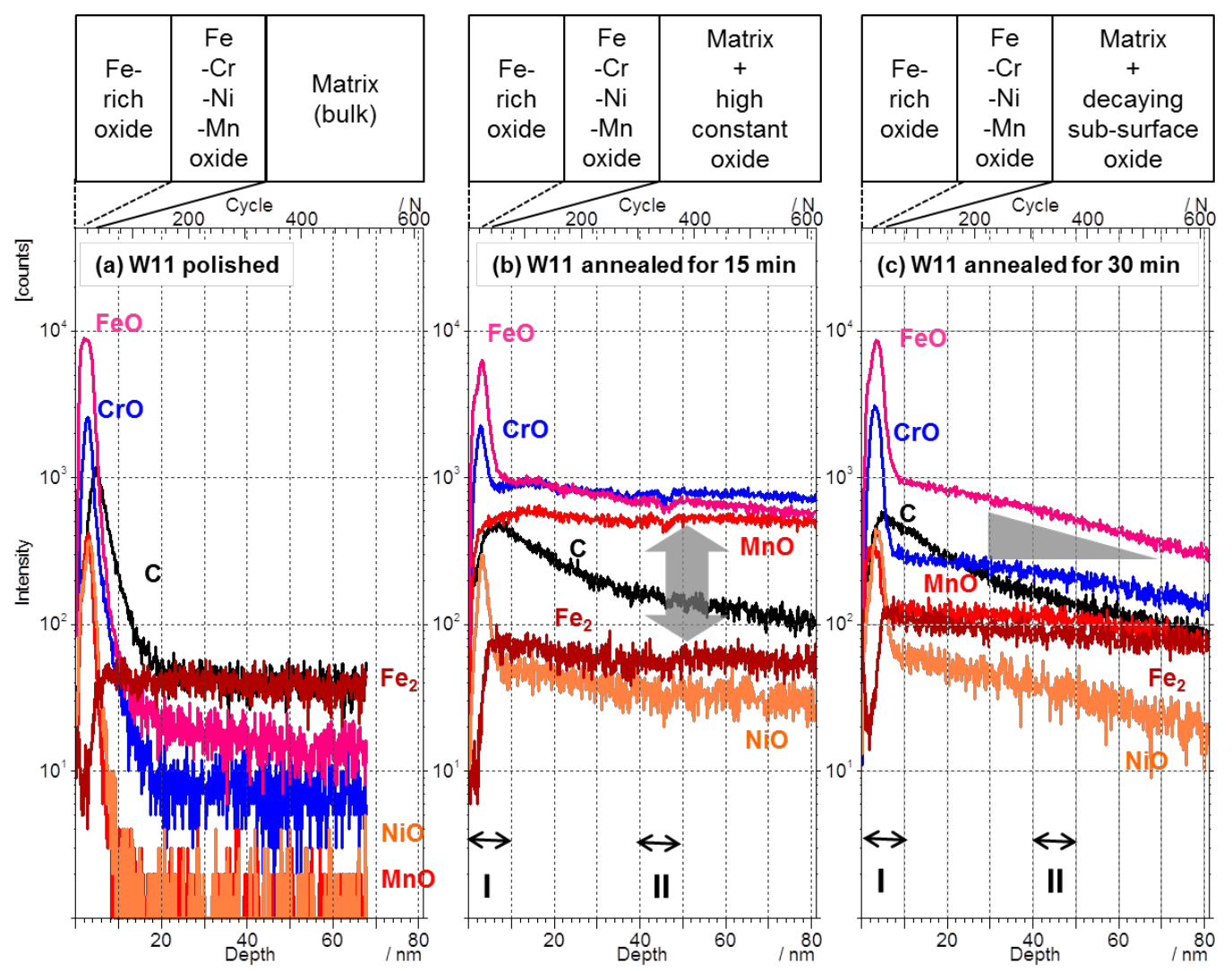

Fig. 4.16 Depth profile on W11. Three types of oxide were found on (a) as polished: thin surface oxide, (b) annealed for 15 min: thin surface oxide + high constant oxide level down to $60 \mathrm{~nm}$ depth and (c) annealed for $30 \mathrm{~min}$ : thin surface oxide + decaying sub-surface oxide.

The secondary ion images of $\mathrm{FeO}, \mathrm{CrO}, \mathrm{NiO}, \mathrm{MnO}, \mathrm{Si}$ and $\mathrm{C}$ on $\mathrm{W} 11$ annealed for $15 \mathrm{~min}$ and for $30 \mathrm{~min}$ are shown in Fig. 4.17. These images were derived for two different characteristic sample depths of (I) 0-10 nm and (II) $40-50 \mathrm{~nm}$. It looks as if a larger grain size is found in the 30 min-sample compared to that of the $15 \mathrm{~min}$ annealed sample. It seems to reflect an enhanced grain growth due to the longer heat treatment time. But, this is not the case. The effect of heat treatment time on the grain size was studied by Martín [20]. According to his results, the average grain size was $47 \mu \mathrm{m}$ and $53 \mu \mathrm{m}$ for $\mathrm{W} 11$ annealed for $15 \mathrm{~min}$ and $30 \mathrm{~min}$, respectively. This indicates just a minor difference of the grain size for these two annealing times. Therefore, it is considered that the SIMS image on 15 min sample (Fig. 4.17 (a)) was taken by chance on an area with a high number of small grains. 
However, the lateral distribution of the oxides such as $\mathrm{CrO}$ and $\mathrm{MnO}$, is affected by the difference in the annealing time. In the sample with solution annealing for $15 \mathrm{~min}$ (Fig. $4.17 \mathrm{a}-\mathrm{I}$ and $\mathrm{a}-\mathrm{II}$ ), $\mathrm{CrO}$ and $\mathrm{MnO}$ show an inhomogeneous distribution in both depths I and II. Bright spots of $\mathrm{CrO}$ and $\mathrm{MnO}$ are significant. This localized enrichment in this sample becomes stronger with depth. Contrary to this, the oxides in W11 after 30 min-solution annealing time distribute homogeneously, as can be seen in Fig. $4.17 \mathrm{~b}$-I and b-II. The color level for oxides is high in grains and grain boundaries.

(a) W11 annealed for $15 \mathrm{~min}$ in graphite heater

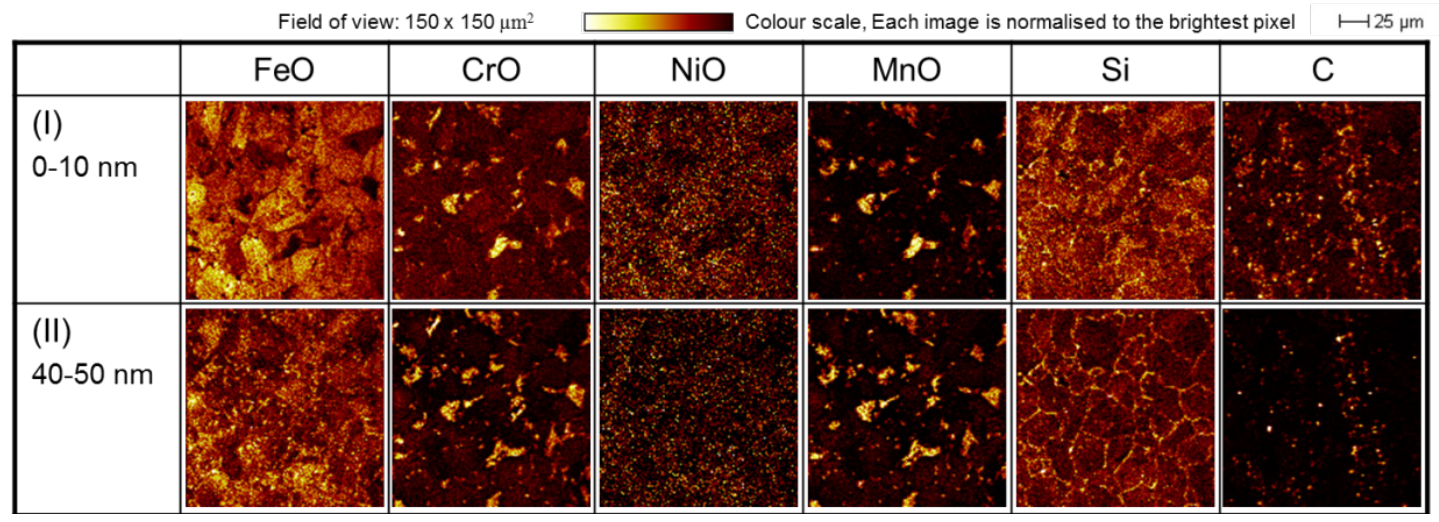

(b) W11 annealed for $30 \mathrm{~min}$ in graphite heater

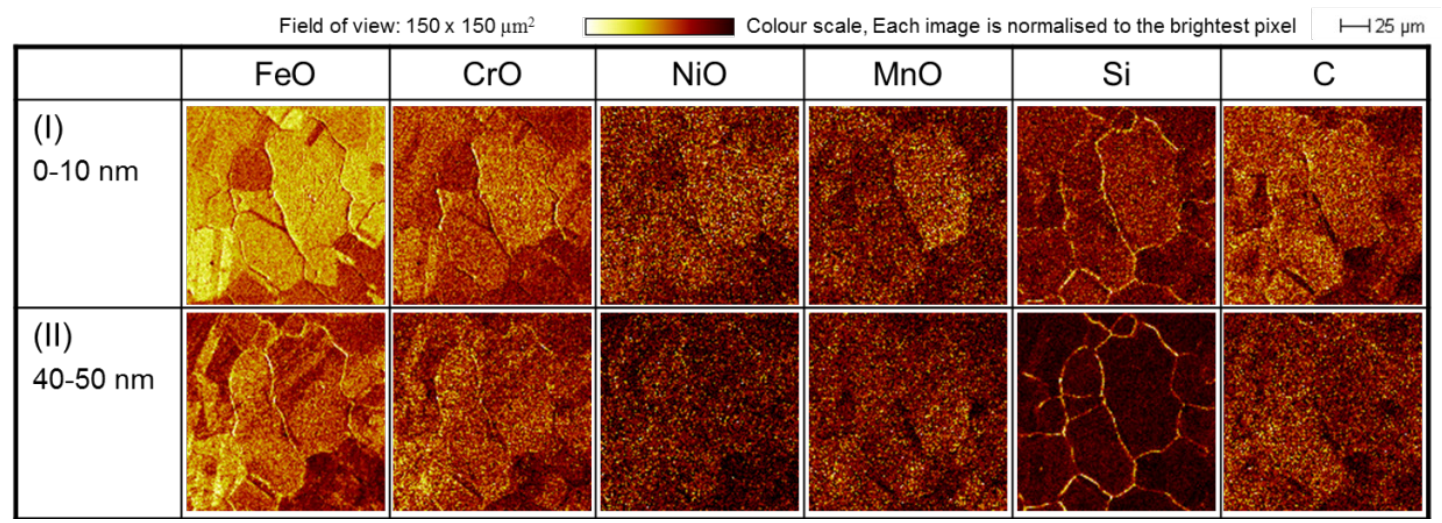

Fig. 4.17 Secondary ion images of W11 annealed for (a) $15 \mathrm{~min}$ and (b) $30 \mathrm{~min}$ at different sample depths as indicated in Fig. 4.16 (b) and (c). CrO and $\mathrm{MnO}$ in W11 solution annealed for 15 min show inhomogeneous distribution while they distribute homogeneously after 30 min heat treatment.

The different results on W11, as solution annealed for $15 \mathrm{~min}$ and for 30 min (Fig. 4.16 and Fig. 4.17), are qualitatively supported by considering the 
thermodynamics of oxidation. According to the Ellingham diagram shown in Fig. 2.16, which plots the standard free energy of formation of an oxide against temperature for the compounds, the oxidation enthalpies of both $\mathrm{Cr}$ and Mn are larger than that of Fe oxide (Fig. 2.16). It is, therefore, expected that $\mathrm{Cr}$ and $\mathrm{Mn}$ oxidize preferentially in case of the sample with 15 min-annealing, as known in terms of 'selective oxidation'. In case of the sample with 30 min-annealing, the selective oxidation occurs as well, but annealing is applied over a longer time span, which might allow the oxide precipitates e.g. $\mathrm{CrO}$ and $\mathrm{MnO}$ to grow as long as oxygen is supplied to the surface. This allows a more homogeneous distribution.

\subsubsection{Measurement of dislocation density by TEM and XRD}

The difference in annealing time also changes the dislocation density in metals. Fig. 4.18 shows TEM micrographs of W11 (a, b) as received (c, d) solution annealed for $15 \mathrm{~min}$, and (e, f) solution annealed for $30 \mathrm{~min}$, at different magnifications. These images were taken in collaboration with $\mathrm{M}$. Deutges. The deformation bands indicated by red arrows and dislocation tangles are observed in the as-received W11 (Fig. $4.18 \mathrm{a}, \mathrm{b}$ ), indicating a high dislocation density. In contrast, the dislocation density is quite low for W11 annealed for $15 \mathrm{~min}$ and $30 \mathrm{~min}$ (Fig. $4.18 \mathrm{c}-\mathrm{f}$ ).

Focusing on the dislocation structure, a planar (grid-like) dislocation distribution and, in some areas, dislocation tangles are found in Fig. 4.18 (c, d). A dislocation pile-up at a grain boundary is observed in Fig. 4.18 (f). W11 (AISI 304L) has a low stacking fault energy (SFE). In general, a lower SFE makes dislocation cross-slip more difficult, resulting in planar arrays of dislocations [120]. The experimental observation result here is in accordance with this interpretation. 

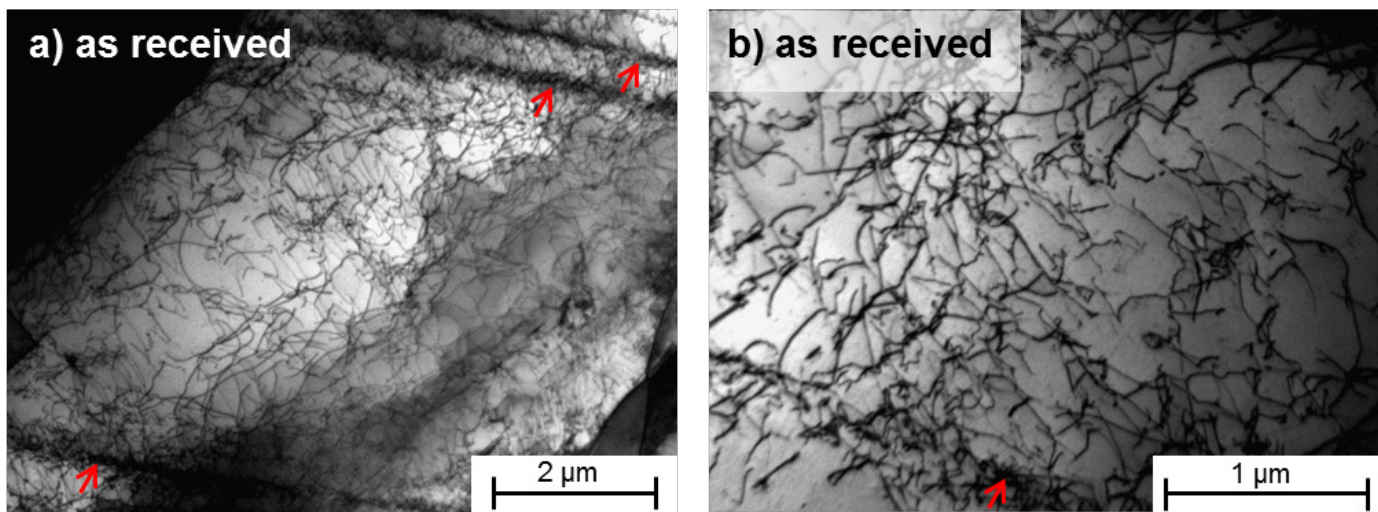

c) for $15 \mathrm{~min}$
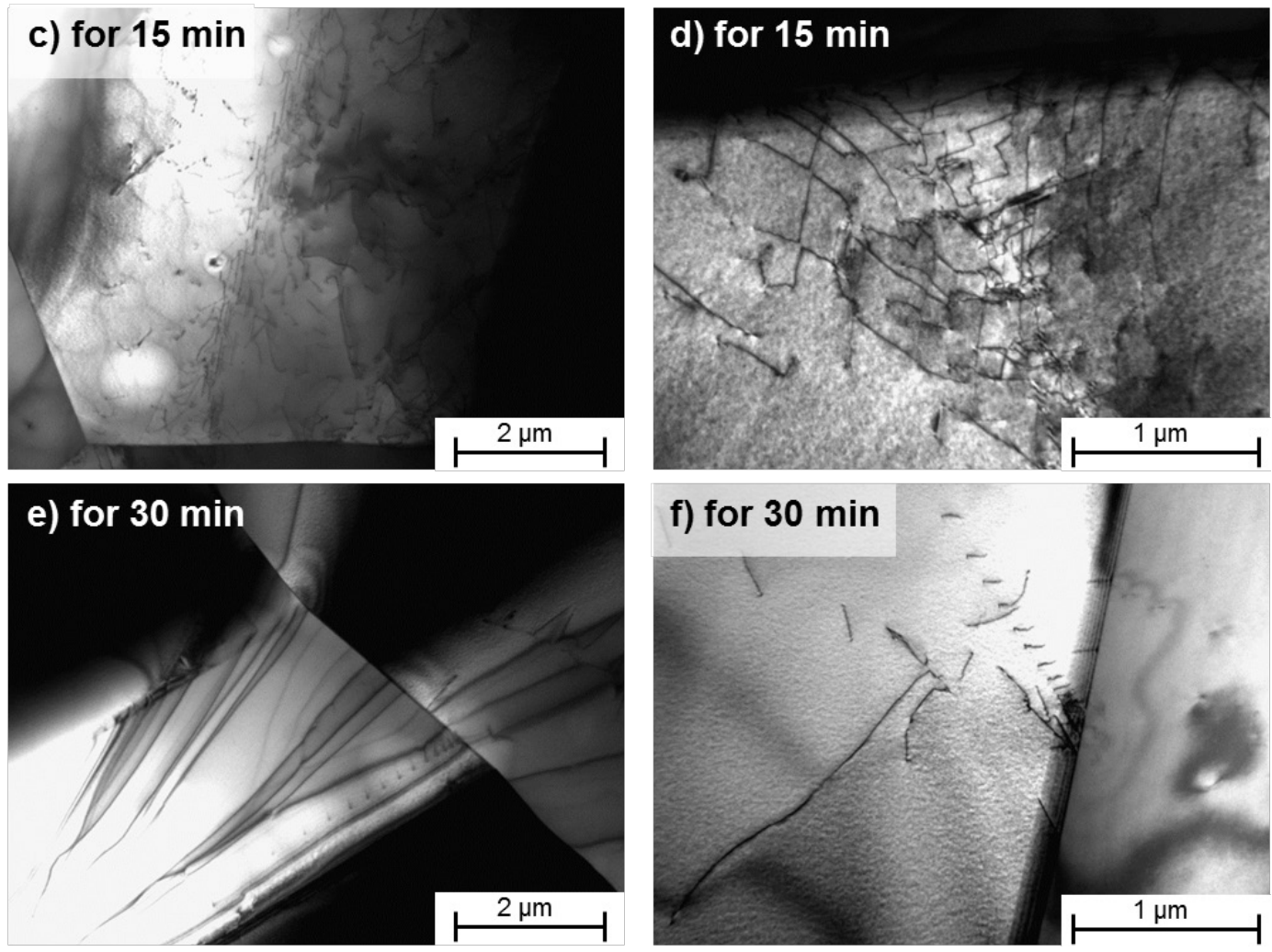

Fig. 4.18 Microstructure of W11 (a),(b) as received (c),(d) solution annealed for 15 $\min (\mathrm{e})$, (f) solution annealed for $30 \mathrm{~min}$, at different magnifications (In collaboration with M. Deutges). (a, b): The deformation bands indicated by red arrows and dislocation tangles are observed in the as-received W11. (c-f) The dislocation density is quite low for W11 annealed for $15 \mathrm{~min}$ and $30 \mathrm{~min}$. The dislocation pile-up at grain boundary can be observed in (f)

The dislocation density seems to decrease with increasing solution annealing time as shown in Fig. 4.18 (c) - (f). These local features qualitatively agree well with the general effect of annealing e.g. recovery and dislocation annihilation. However, it is difficult to evaluate the dislocation density by TEM since it is of limited statistical significance due to the small observation area. Further, the number of observable dislocation may be 
changed by the diffraction contrast depending on the sample tilt.

Alternatively, the XRD peak broadening method (section 3.3.3) was employed to determine the dislocation density. This method allows measuring a large sample volume. Table 4.3 shows the dislocation density as obtained by the XRD peak analysis.

Table 4.3 Dislocation density obtained by XRD

\begin{tabular}{|c|c|}
\hline & Dislocation density $\left(1 / \mathrm{m}^{2}\right)$ \\
\hline As received & $2.7 \times 10^{12}$ \\
\hline for $15 \mathrm{~min}$ & $3.4 \times 10^{12}$ \\
\hline for $30 \mathrm{~min}$ & $1.7 \times 10^{11}$ \\
\hline
\end{tabular}

The as received W11 and W11 solution annealed for 15 min exhibit the almost identical dislocation density of $10^{12} \mathrm{~m}^{-2}$, while the dislocation density in W11 annealed for $30 \mathrm{~min}$ is $10^{11} \mathrm{~m}^{-2}$. In general, the dislocation density of

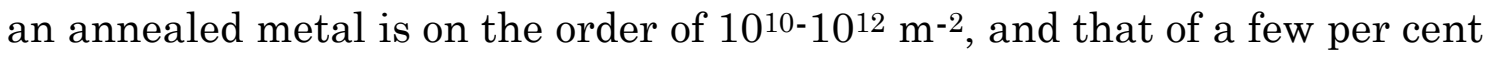

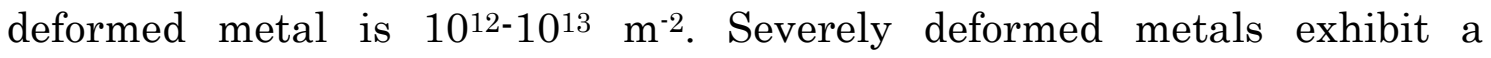

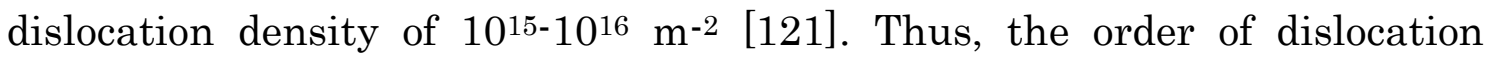
density obtained by XRD is in good agreement with the value reported so far, for an annealed sample.

It might seem to be a contradiction that the dislocation density of as-received W11 is almost equivalent to that of W11 annealed for $15 \mathrm{~min}$ while TEM observation reveals a large difference between them. But, in case of the as-machined W11, it can be assumed that there is a dislocation distribution gradient from the edge to the center of the sample. The dislocation density could be much lower in the center region than in the vicinity of an edge where many defects are introduced by the wet turning process. However, the dislocation density was obtained over the whole sample area by XRD. As a consequence, XRD measurements may not reveal the difference between W11 in the as machined condition and in the condition when annealed for $15 \mathrm{~min}$. 


\subsection{Low-nickel steels: Tensile test at $-50^{\circ} \mathrm{C}$}

\subsubsection{Tensile tests results}

The low-nickel stainless steels and reference stainless steels (W11 and W12) were tensile tested at $-50{ }^{\circ} \mathrm{C}$ in air at atmospheric pressure and in hydrogen atmosphere at $40 \mathrm{MPa}$. Again, all specimens were solution annealed by graphite heater at $1050{ }^{\circ} \mathrm{C}$ or $1150{ }^{\circ} \mathrm{C}$.

In the previous section, the tensile tests were performed on W11 at room temperature while the lower test temperature was used in this section. In order to reveal the temperature dependencies of stress-strain behavior, the tensile tests parameters obtained at room temperature and at $-50{ }^{\circ} \mathrm{C}$ in air and in hydrogen are compared in Fig. 4.19. The data was obtained from Martín [20]. The $0.2 \%$ offset yield strength $\left(\sigma_{0.2 \% y}\right)$ and ultimate tensile strength $\left(\sigma_{U T S}\right)$ indicate the strength of materials while elongation to fracture $(\Delta l)$ and reduction area (RA) represent the ductile properties.

As can be seen in this result (Fig. 4.19), the difference in test temperature in air exhibits a significant impact on the ultimate tensile strength $\sigma_{U T S}$. Namely, a large reduction of ductility is found in $\mathrm{H}_{2}$ at $-50{ }^{\circ} \mathrm{C}$. For more detail, the RA and RRA values of all of the low-nickel steels are compared in Table 4.4 [20]. Remarkable HE-resistant properties (high RRA) are found for the samples W12 and W19. 


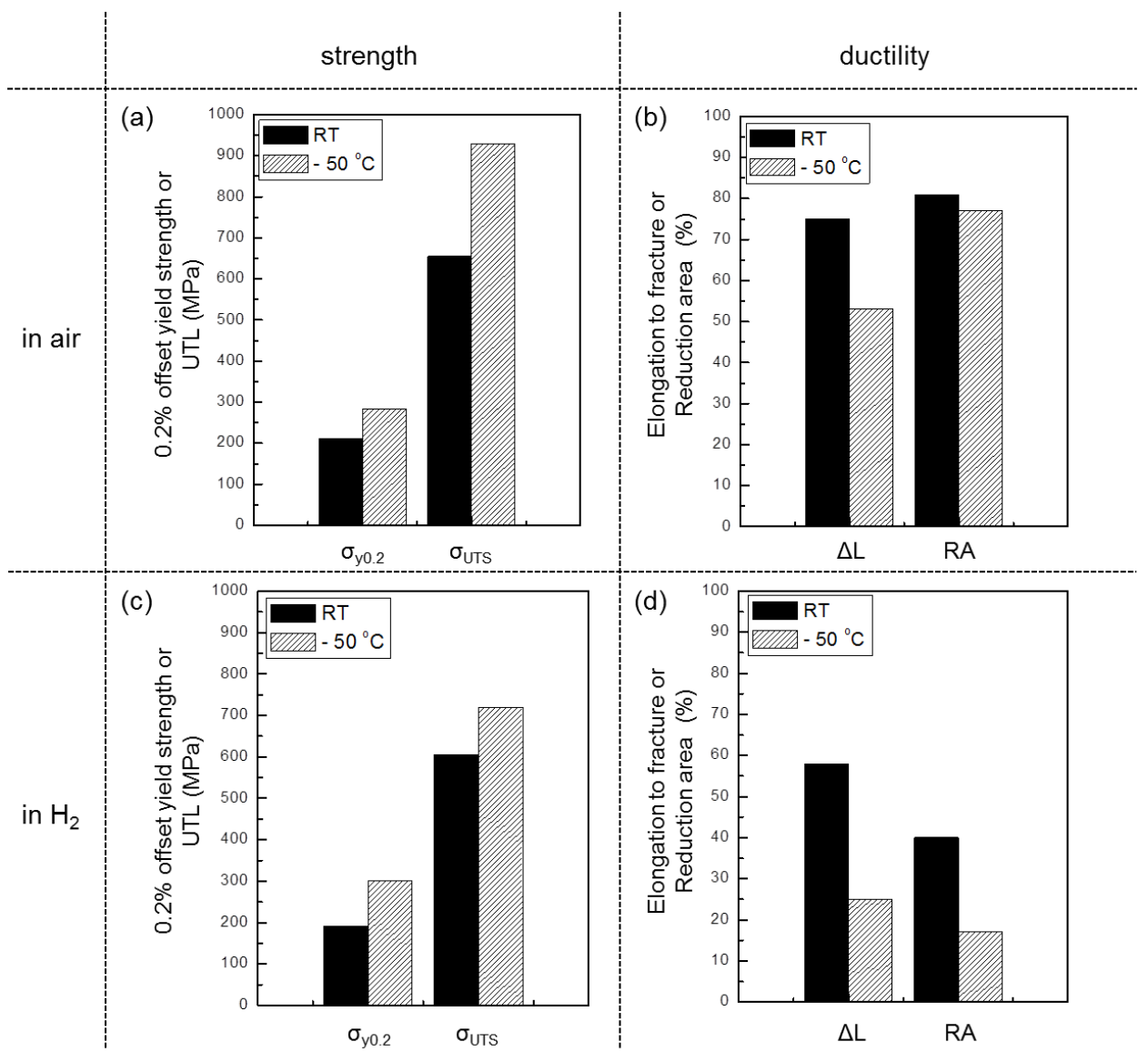

Fig. 4.19 The parameters provided from tensile tests at room temperature and at $-50{ }^{\circ} \mathrm{C}$. (a) and (c): $0.2 \%$ offset yield strength $\left(\sigma_{\mathrm{y} 0.2}\right)$ and ultimate tensile strength $\left(\sigma_{\mathrm{UTS}}\right)$. (b) and (d): elongation to fracture $(\Delta \mathrm{L})$ and reduction area (RA). The difference in test temperature in air exhibits the significant impact on ultimate tensile strength $\sigma_{U T S}$. Large reduction of ductility is found in $\mathrm{H}_{2}$ at $-50{ }^{\circ} \mathrm{C}$. (Data from Martín [20], with permission of the author.)

Table 4.4 RA and RRA values of W11-W21 solution annealed for 30 min by graphite furnace. Tensile tests were peformed at $-50{ }^{\circ} \mathrm{C}$ in air at atmospheric pressure and in hydrogen atmosphere at $40 \mathrm{MPa}$ [20] The results on W11 tested at room temperature is presented for comparison. (Data from Martín [20], with permission of the author.)

\begin{tabular}{ccccccc}
\hline Alloy & \multicolumn{2}{c}{ Annealing } & Tensile test & \multicolumn{2}{c}{ RA (\%) } & RRA (\%) \\
& Time (min) & Temp. $\left({ }^{\circ} \mathrm{C}\right)$ & Temp. $\left({ }^{\circ} \mathrm{C}\right)$ & air & $\mathrm{H}_{2}$ & \\
\hline W11 & 30 & 1050 & $\mathrm{RT}$ & 81 & 40 & 49 \\
W11 & 30 & 1050 & -50 & 77 & 17 & 23 \\
W12 & 30 & 1050 & -50 & 80 & 75 & 93 \\
W14 & 30 & 1050 & -50 & 73 & 9 & 13 \\
W15 & 30 & 1050 & -50 & 74 & 15 & 20 \\
W16 & 30 & 1050 & -50 & 67 & 22 & 34 \\
W17 & 30 & 1050 & -50 & 71 & 32 & 45 \\
W18 & 30 & 1050 & -50 & 71 & 15 & 21 \\
W19 & 30 & 1050 & -50 & 83 & 69 & 83 \\
W20 & 30 & 1150 & -50 & 73 & 55 & 75 \\
W21 & 30 & 1150 & -50 & 76 & 30 & 39 \\
\hline
\end{tabular}


Fig. 4.20 shows RRA values plotted as a function of the Ni content of the low-nickel steels. Results of W11 annealed for $30 \mathrm{~min}$ tensile tested at room temperature are also plotted for comparison.

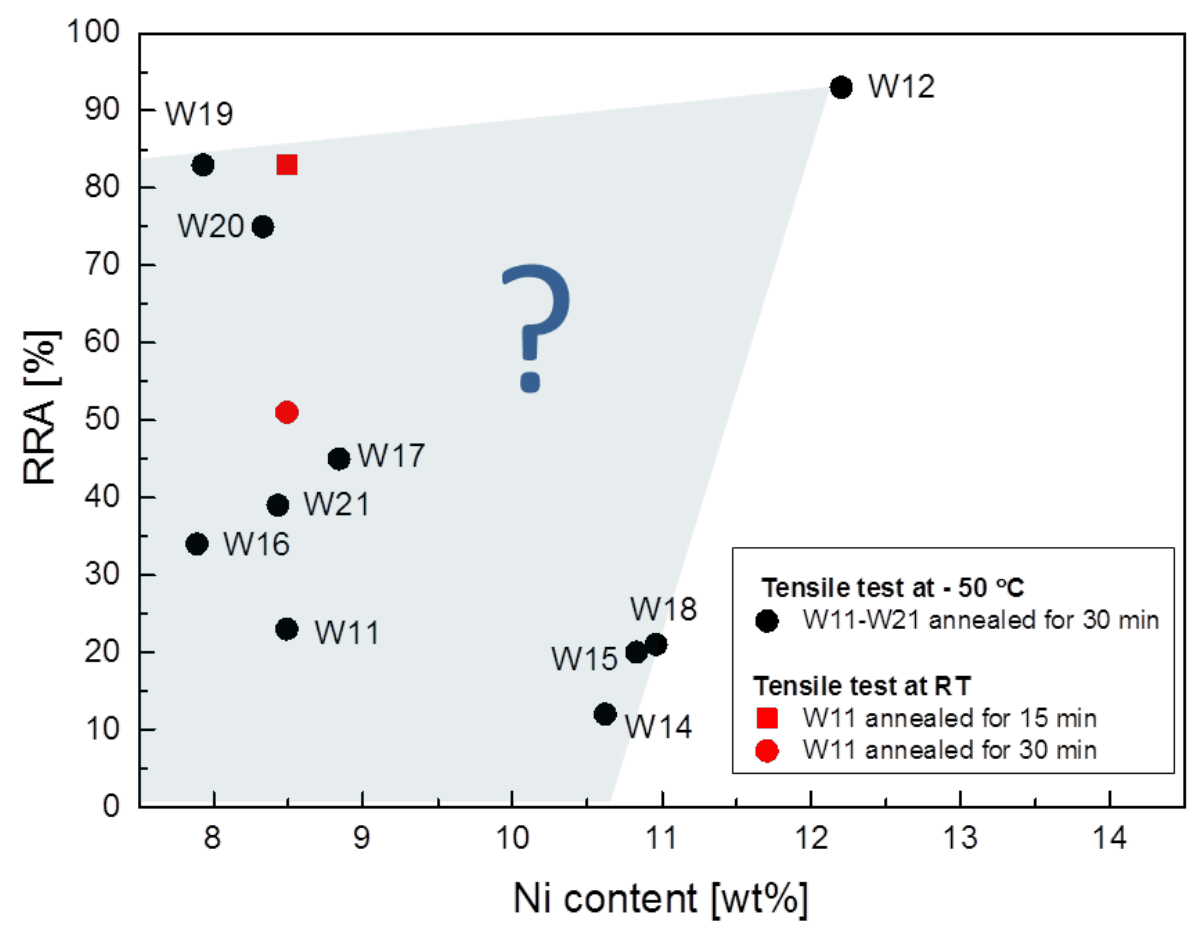

Fig. 4.20 Relative reduction of area (RRA) plotted as a function of the Ni content of the austenitic stainless steel. Tensile tests were performed $-50{ }^{\circ} \mathrm{C}$ in air at atmospheric pressure and in hydrogen atmosphere at $40 \mathrm{MPa}$. Results of W11 annealed for $15 \mathrm{~min}$ and $30 \mathrm{~min}$ tensile tested at RT are plotted for the comparison. Large scatter was found also for the low-nickel austenitiec stainless steels W11-W21.

The RRA-values of all samples, tested at $-50{ }^{\circ} \mathrm{C}$ and plotted versus the $\mathrm{Ni}$ content, are widely scattered. This scatter is attributed to the large difference in the chemical composition. In general, the austenite stability is strongly influenced by the alloying elements. On one hand, alloying elements such as $\mathrm{C}, \mathrm{N}, \mathrm{Mn}$ and $\mathrm{Cu}$ stabilize the austenite phase, as $\mathrm{Ni}$ does. On the other hand, alloying elements such as Al, Si, P, Ti, V and Mo stabilize the ferrite phase [20].

Here, we should note again that the composition given in Fig. 4.20 is nominal composition. Surface and near surface chemical conditions might be significantly different, due to oxidation reaction and concomitant modification of bulk composition. To track down this aspect, SIMS analysis was carried out for all of the samples. 


\subsubsection{SIMS analysis and EDX}

\section{- Depth Profile analysis}

SIMS analysis was performed on the new alloys. The oxide layers formed on 10 different alloys were characterized into four types by the shape of the profiles. The classified profiles are shown in Fig. 4.21.

The classification of the oxide layer is as follows,
(a) thick oxide:
W14 and W19
(b) decaying sub-surface oxide:
W11, W16, W20 and W21,
(c) thin surface oxide:
W15 and W18
(d) high constant oxide level:
W12 and W17

(a) thick oxide
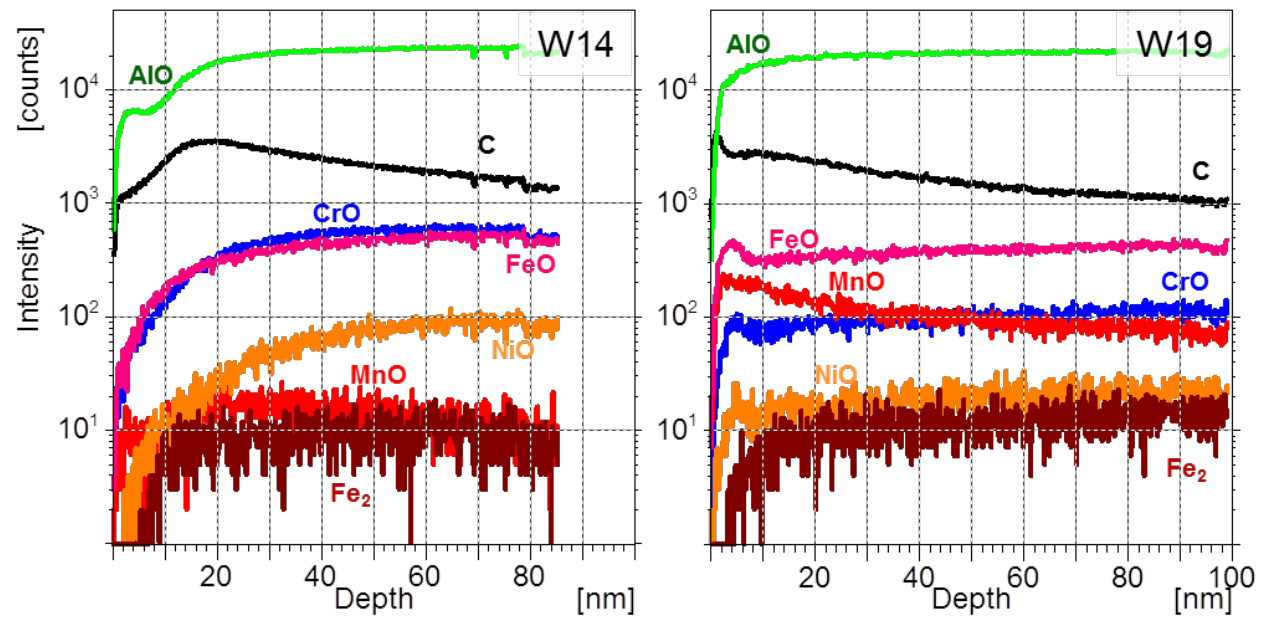

(b) decaying sub-surface oxide
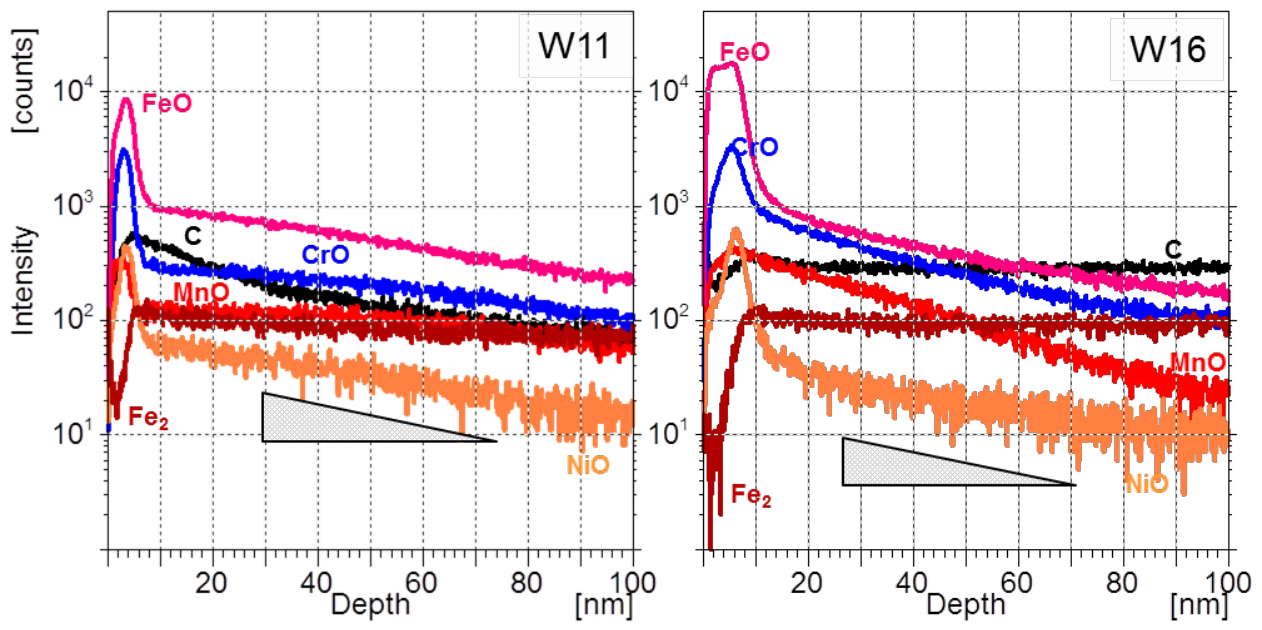

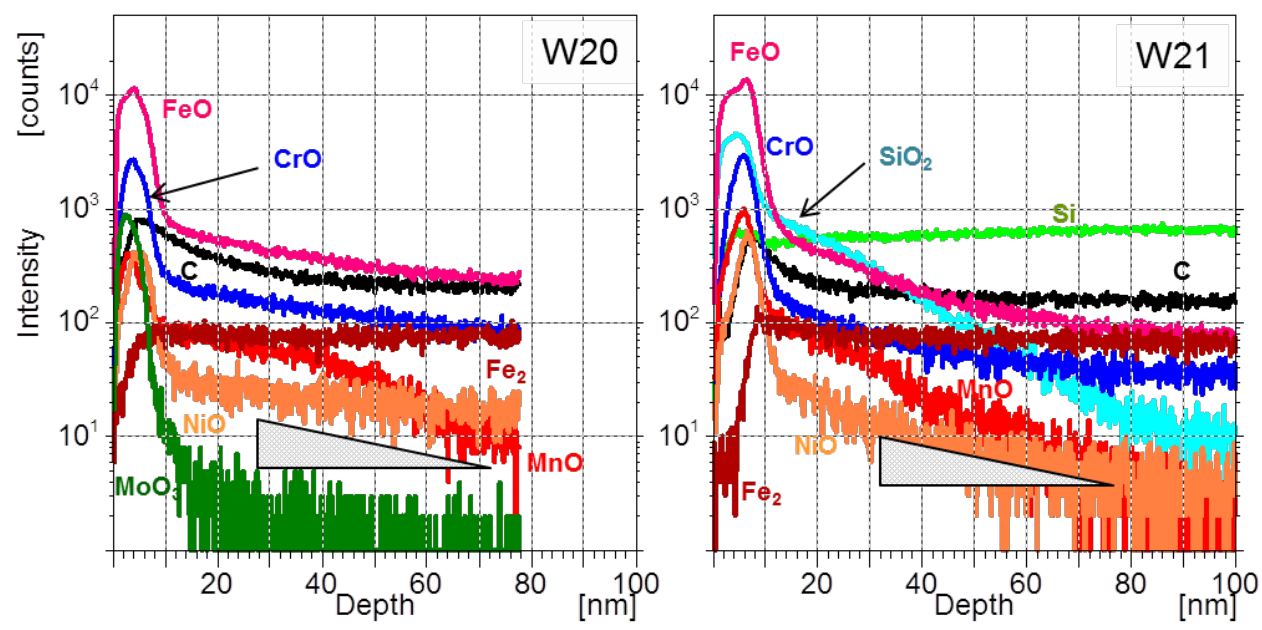

(c) thin surface oxide
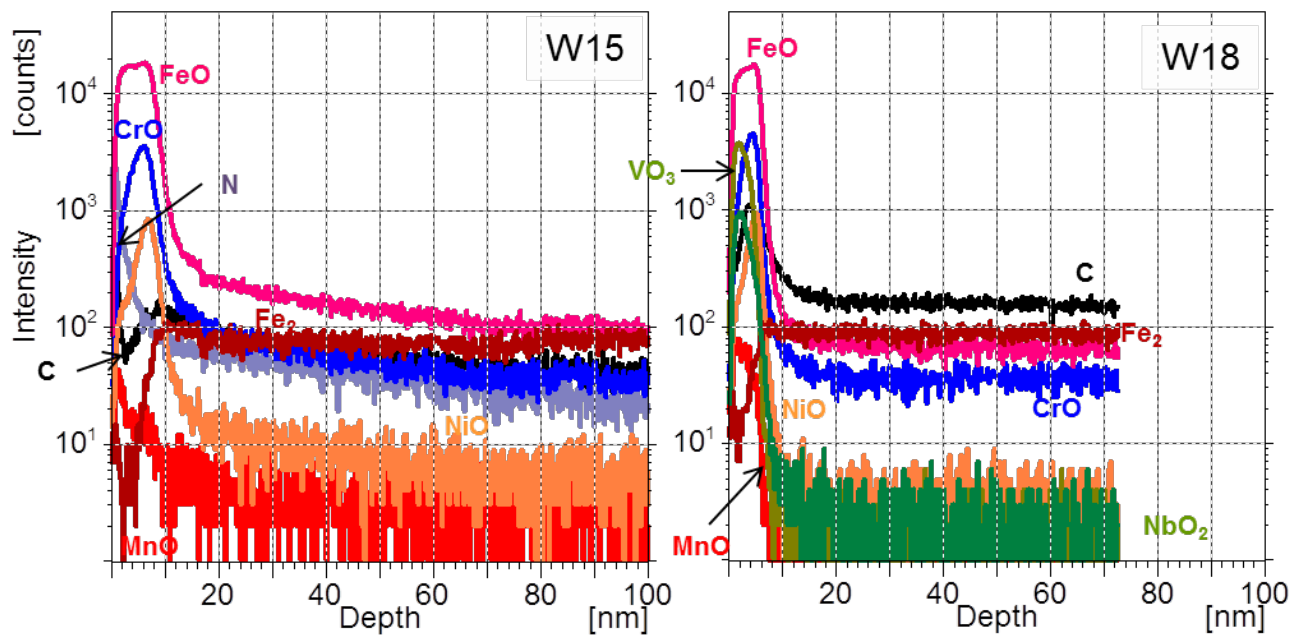

(d) high constant oxide level
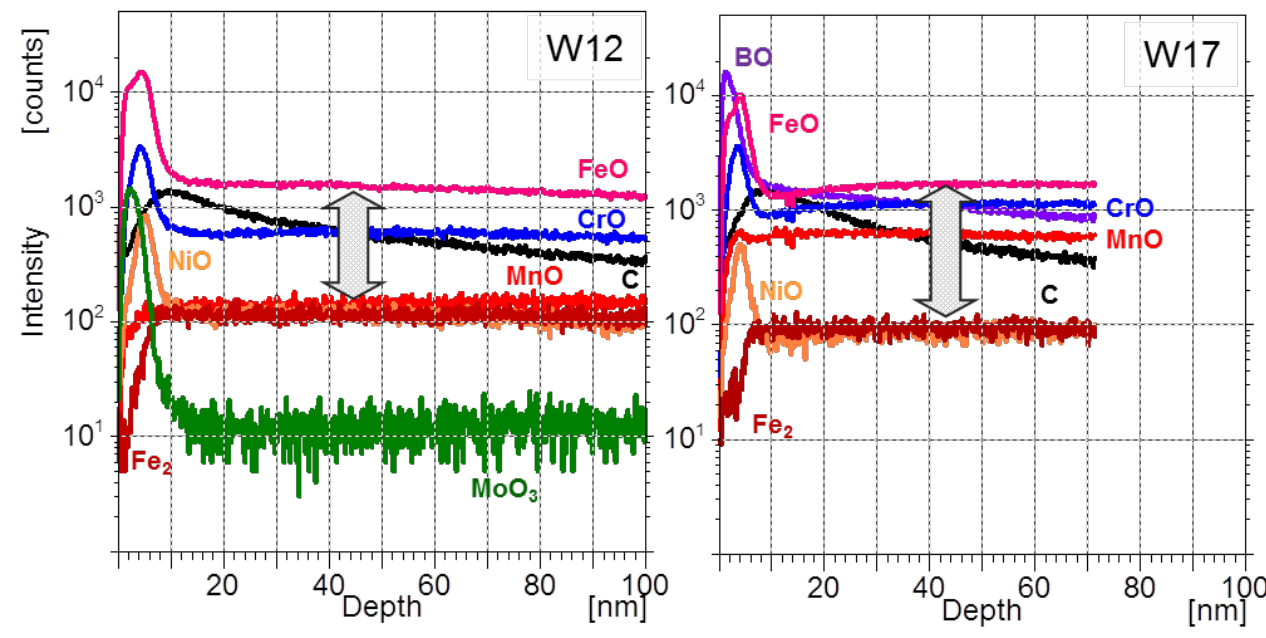

Fig. 4.21 The type of oxide layer formed on W11-W21 annealed for 30 min by graphite furnace. (a) Thick oxide (W14 and W19), (b) thin surface oxide and decaying sub-surface oxide (W11, W16, W20 and W21),(c) thin surface oxide (W15 and W18) and (d) thin surface oxide and high constant oxide level down to $60 \mathrm{~nm}$ depth (W12 and W17). 
The meaning of each oxide layer type is summarized as follows. (a) In W14 and W19, a thick oxide containing Al was found. (b) In W11, W16, W20 and $\mathrm{W} 21$, the signal of $\mathrm{FeO}$ and $\mathrm{CrO}$ gradually decreases to the same intensity level of $\mathrm{Fe}_{2}$, which reflects iron metal in bulk, with increasing depth. This type of oxide is named as "decaying sub-surface oxide". (c) Thin oxide layer in W15 and W18, which is named as "thin surface oxide", the signal of $\mathrm{FeO}$ and $\mathrm{CrO}$ instantly reaches the same level as $\mathrm{Fe}_{2}$ after passing the thin oxide region. (d) In $\mathrm{W} 12$ and $\mathrm{W} 17$, the signal of $\mathrm{FeO}$ and $\mathrm{CrO}$ maintains a constant level and this level is considerably higher than that of $\mathrm{Fe}_{2}$. Thus, this oxide layer is named as "high constant oxide level". The long depth profile measurements were conducted on W12 and W17. The signal of FeO and $\mathrm{CrO}$ in $\mathrm{W} 12$ and $\mathrm{W} 17$ intersects with $\mathrm{Fe}_{2}$ at $600 \mathrm{~nm}$ and at $1 \mu \mathrm{m}$ in depth, respectively. The long depth profile results can be found in Appendix 8.3. It should be noted that (b) decaying sub-surface oxide and (d) high constant oxide level also have a thin surface oxide on top.

All alloys (except W14 and W19) were measured at least three times at different sample positions in order to confirm the repeatability of the depth profile. Similar profiles were obtained in all cases. The repeatability of measurements can be confirmed in Appendix 8.4.

The oxide layer stacking comprised of different oxides is found in most of the samples except for W14 and W19. Alloying elements of Mo, B, V, Nb and $\mathrm{Si}$ are found in the outer layer. The stacking sequence of $\mathrm{MnO}$ differs from sample to sample. $\mathrm{MnO}$ is found in the outer layer of W11, W20 and W21 while the considerably broad profile of $\mathrm{MnO}$ was found in W16. Moreover, $\mathrm{MnO}$ distributes homogeneously in W12 and W17.

Remarkably, the thick oxide layer of W14 and W19 contains relatively high amount of $\mathrm{Al}$. The aluminum content of W14 and W19 is $1.64 \mathrm{wt} \%$ and 2.87 wt\%, respectively. The SIMS measurement cannot reach the interface region of these alloys because of the thick oxide. Therefore, cross sectional EDX observation was performed on W19 as shown in Fig. 4.22.

As can be seen in Fig. 4.22, the aluminum oxide layer continues into the depth of at least $150 \mathrm{~nm}$ in W19. The formation of this considerably thick 
oxide can be explained by selective oxidation. The selective oxidation of aluminum is a well known phenomenon and often used in high temperature applications. Small amounts of $\mathrm{Al}$ additive cover the alloy surface in the form of Al-oxide (scale) and this dense Al-oxide improves the high temperature corrosion resistance of alloys [122].
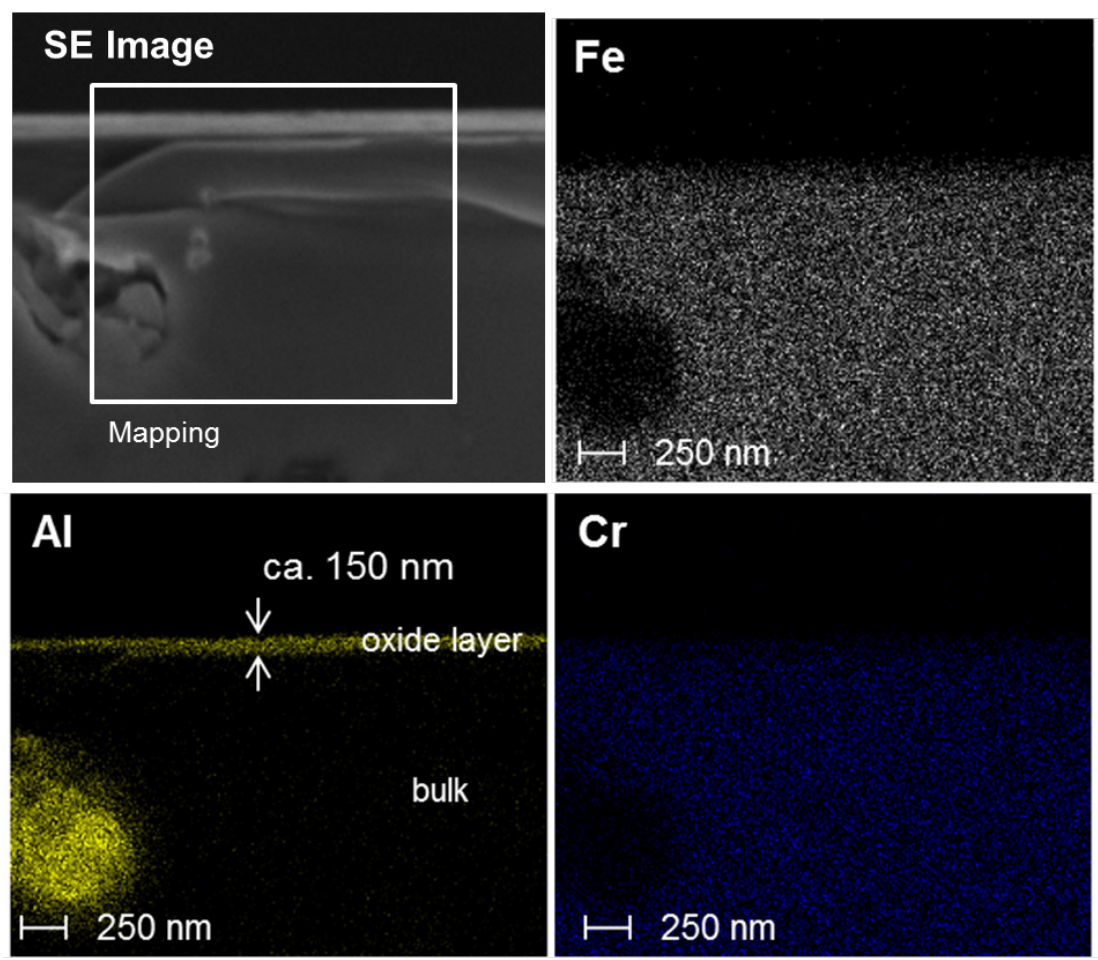

Fig. 4.22 Cross sectional EDX observation on W19. EDX measurement was peformed in the rectangle region indicated in SEM image. Oxide layer thickness is ca. $150 \mathrm{~nm}$

\section{- Secondary Ion Image analysis}

The secondary ion images of (a) thick oxide layer (W14 and W19 annealed for $30 \mathrm{~min}$ ) are shown in Fig. 4.23. The oxide layer of these samples is homogeneous in the depth direction. Therefore, the images summed over all depths are shown in Fig. 4.23.

In $\mathrm{W} 14, \mathrm{AlO}$ and $\mathrm{C}$ distribute homogeneously. $\mathrm{FeO}, \mathrm{CrO}, \mathrm{NiO}$ and $\mathrm{MnO}$ show some vertical dark lines, presumably resulting from the polishing before the solution annealing. In W19, AlO distributes homogeneously as well as in $\mathrm{W} 14$, but an inhomogeneous distribution of $\mathrm{FeO}, \mathrm{CrO}, \mathrm{NiO}, \mathrm{MnO}$ and $\mathrm{C}$ is clearly found. 


\begin{tabular}{l} 
Field of view: $150 \times 150 \mathrm{\mu m}^{2}$ \\
\hline
\end{tabular}

Fig. 4.23 Secondary ion images of W14 and W19. In W19, FeO, CrO, NiO, MnO and $\mathrm{C}$ show island distribution.

Fig. 4.24 (a) shows the optical images of W19 at two different magnifications. At lower magnification, many dark lines are observed. The optical image magnified square section indicates that the dark lines contain many particles with the size of about 1-2 $\mu \mathrm{m}$, appearing as white spots.

In order to obtain the chemical information of the dark and light region in Fig. 4.24 (a), the secondary ion image was acquired at a higher magnification $(80 \times 80 \mu \mathrm{m})$ in the green rectangle region shown in Fig. 4.24 (b). This image was obtained after the depth profile measurement shown in Fig. 4.22. The result is represented in Fig. 4.24 (c) on several oxide species. $\mathrm{FeO}, \mathrm{CrO}, \mathrm{MnO}, \mathrm{NiO}$ and $\mathrm{C}$ appear to be colocalized, while $\mathrm{AlO}$ distributes relatively homogeneous. In this $\mathrm{AlO}$ image, many dark spots with the size of 1-2 $\mathrm{mm}$ (indicated by arrows) are observed, as indicated by arrows. These dark spots correspond to the particles within the dark region in Fig. 4.24 (a). To elucidate this interesting feature more clearly, a color overlay of $\mathrm{AlO}$ and $\mathrm{FeO}$ was carried out and shown in Fig. 4.24 (d). In this picture, red color denotes the presence of $\mathrm{AlO}$, blue color denotes the presence of $\mathrm{FeO}$, and purple color denotes the presence of $\mathrm{AlO}$ and FeO. Obviously, the small particles are rich in $\mathrm{FeO}$ and deficient in $\mathrm{AlO}$. Moreover, the dark region in optical micrograph is assigned to be a mixed phase of $\mathrm{AlO}, \mathrm{FeO}, \mathrm{CrO}, \mathrm{NiO}$, $\mathrm{MnO}$ and $\mathrm{C}$, while the light region mainly consists of $\mathrm{AlO}$. 
(a)

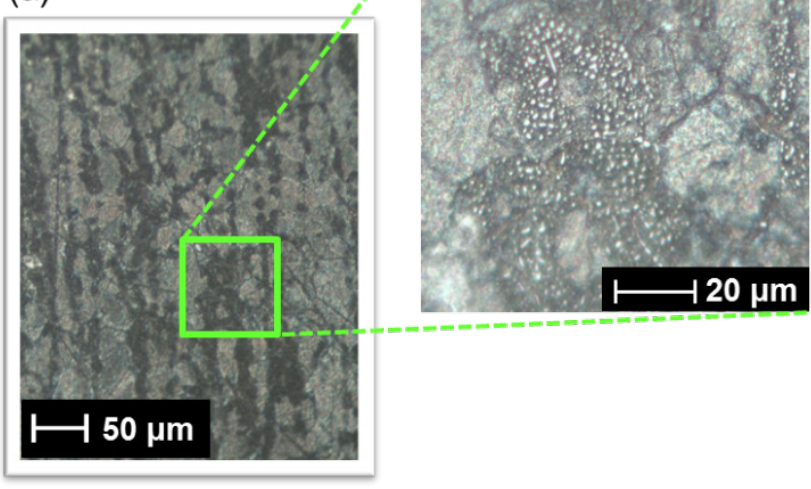

(b)

Field of view : $150 \times 150 \mu \mathrm{m}^{2}$

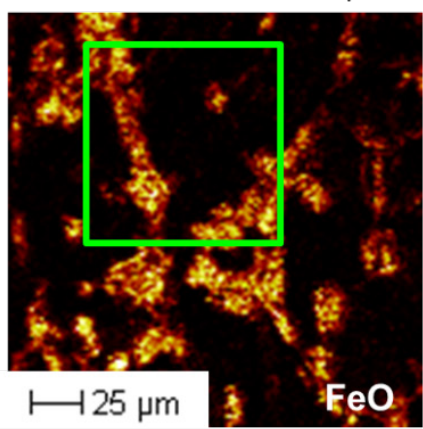

(d)

(c) Field of view: $80 \times 80 \mu \mathrm{m}^{2}$

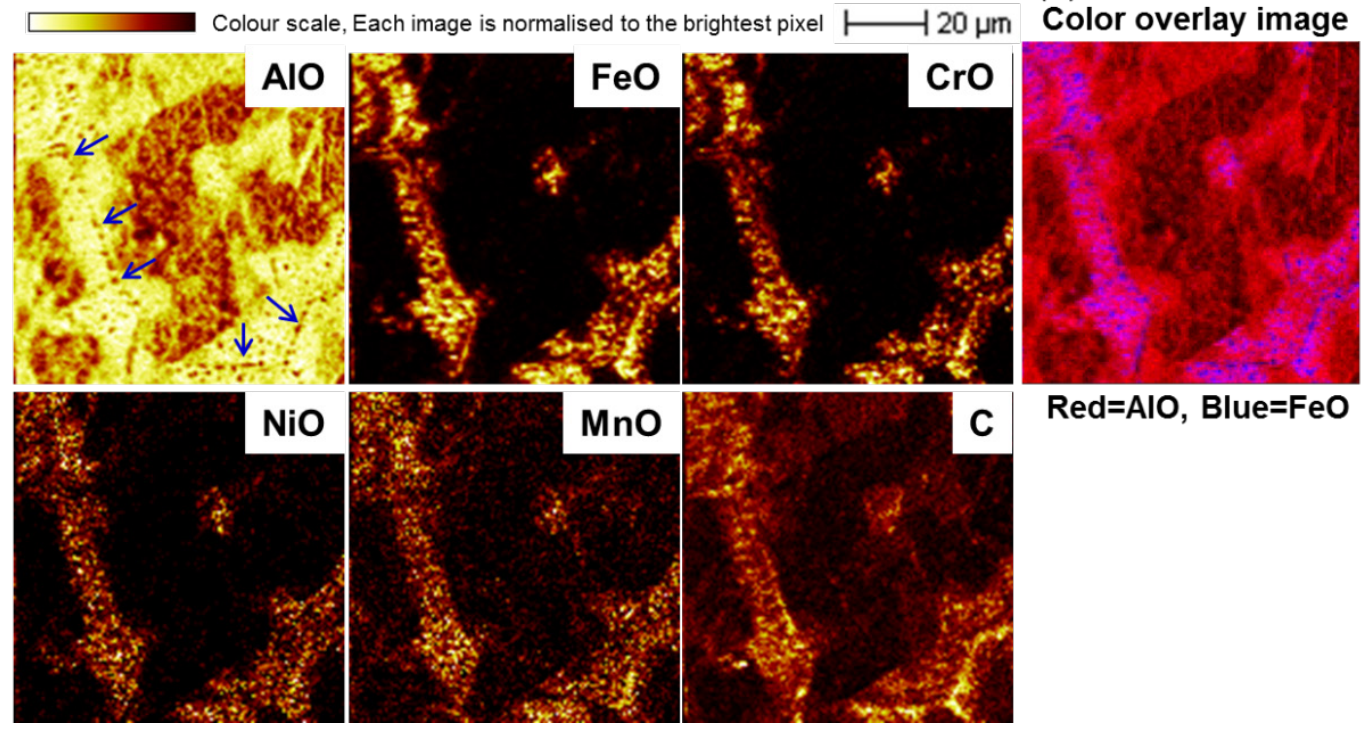

Fig. 4.24 (a) Optical images of W19 at two different magnifications. (b) FeO image. The green rectangle indicates the analytical area of (c). (c) Secondary ion image of W19 at higher magnification $(80 \times 80 \mu \mathrm{m})(\mathrm{d})$ Color overlay image of $\mathrm{AlO}$ and $\mathrm{FeO}$.

(a) The dark line containing particles can be seen. (c) $\mathrm{FeO}, \mathrm{CrO}, \mathrm{MnO}, \mathrm{NiO}$ and $\mathrm{C}$ appear to be colocalized. In AIO image, many dark spots corresponding to the particles within dark region in (a) can be observed. (d) The particles are rich in $\mathrm{FeO}$ and deficient in $\mathrm{AIO}$. The dark region in optical microsope is the mixed phase of $\mathrm{AIO}, \mathrm{FeO}, \mathrm{CrO}, \mathrm{NiO}$, $\mathrm{MnO}$ and $\mathrm{C}$, while the light region mainly consists of $\mathrm{AIO}$.

Such strong localization of oxides was not found in the other types of oxides. FeO ion images of (a)decaying sub-surface oxide (b) thin oxide and (c) high constant oxide level at different sample depths are summarized in Fig. 4.25. The distribution of the other elements is found in Appendix 8.5. 
$\longmapsto 25 \mu \mathrm{m}$

Field of view: $150 \times 150 \mu \mathrm{m}^{2} \quad$ Colour scale, Each image is normalised to the brightest pixel

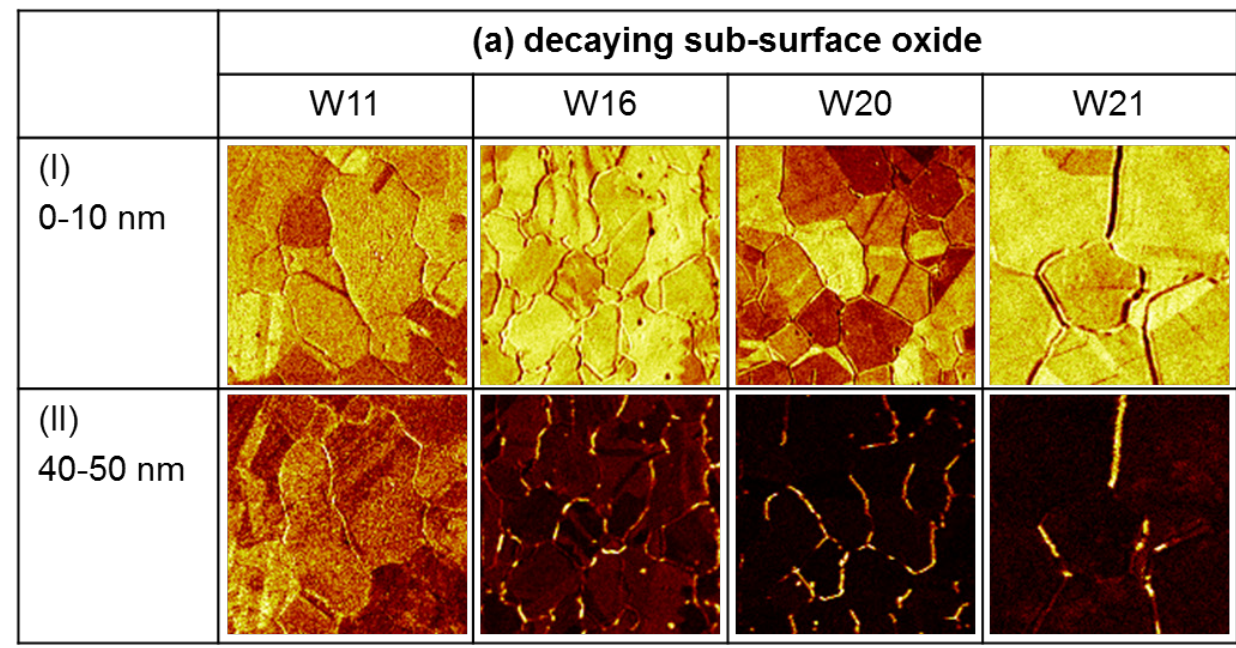

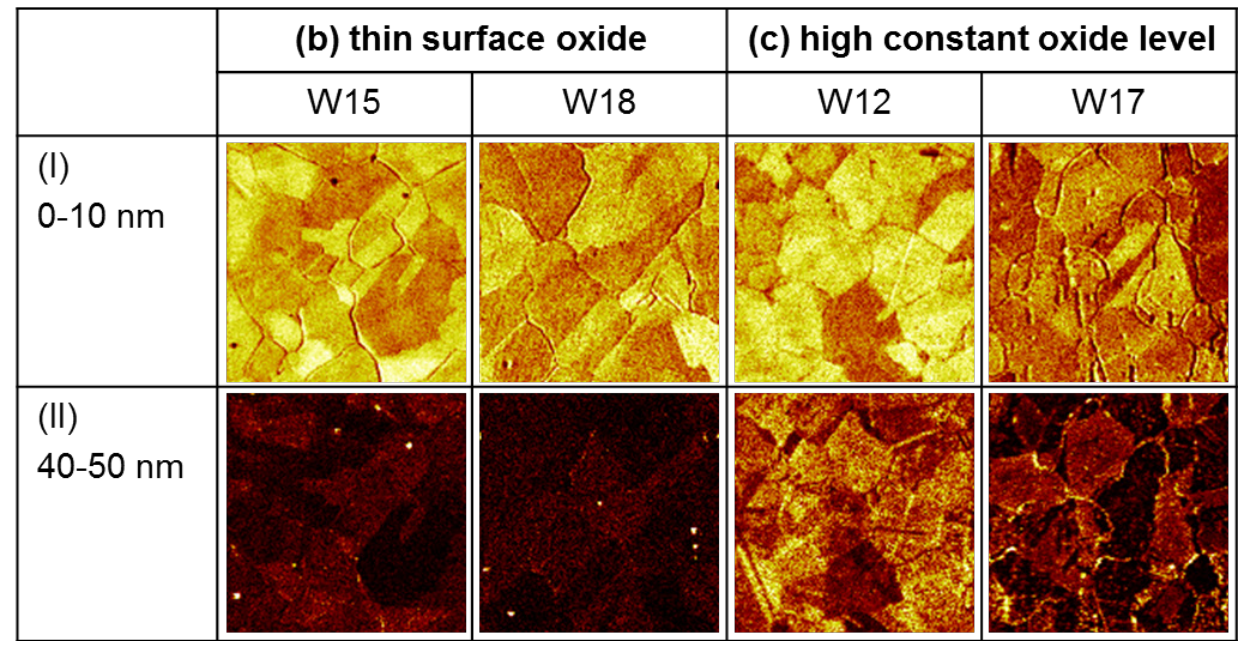

Fig. 4.25 FeO ion images of (a)decaying sub-surface oxide (b) thin surface oxide and (c) high constant oxide level at different sample depths. At 40-50 nm, $\mathrm{FeO}$ is found along grain boundaries in W16, W20 and W21.

Unlike the case of W 14 and W19 (Fig. 4.24), for all the samples shown here, $\mathrm{FeO}$ is distributed homogeneously at the depth of 0-10 $\mathrm{nm}$. At the depth of 40-50 nm, FeO is found along grain boundaries in W16, W20 and W21, while FeO distributes relatively homogeneous in W11, W15, W18, W12 and W17.

\section{- Depth profile analysis on polished steels.}

As shown in Fig. 4.21, four types of oxide layers were found on the new alloys, in annealed condition. For purpose of comparison, SIMS analysis were conducted on these alloys in polished conditions. The polishing was 
carried out mechanically at room temperature.

Fig. 4.26 exemplarily shows SIMS depth profiles on polished W19, W11, W15 and W12. All results can be found in Appendix 8.5..
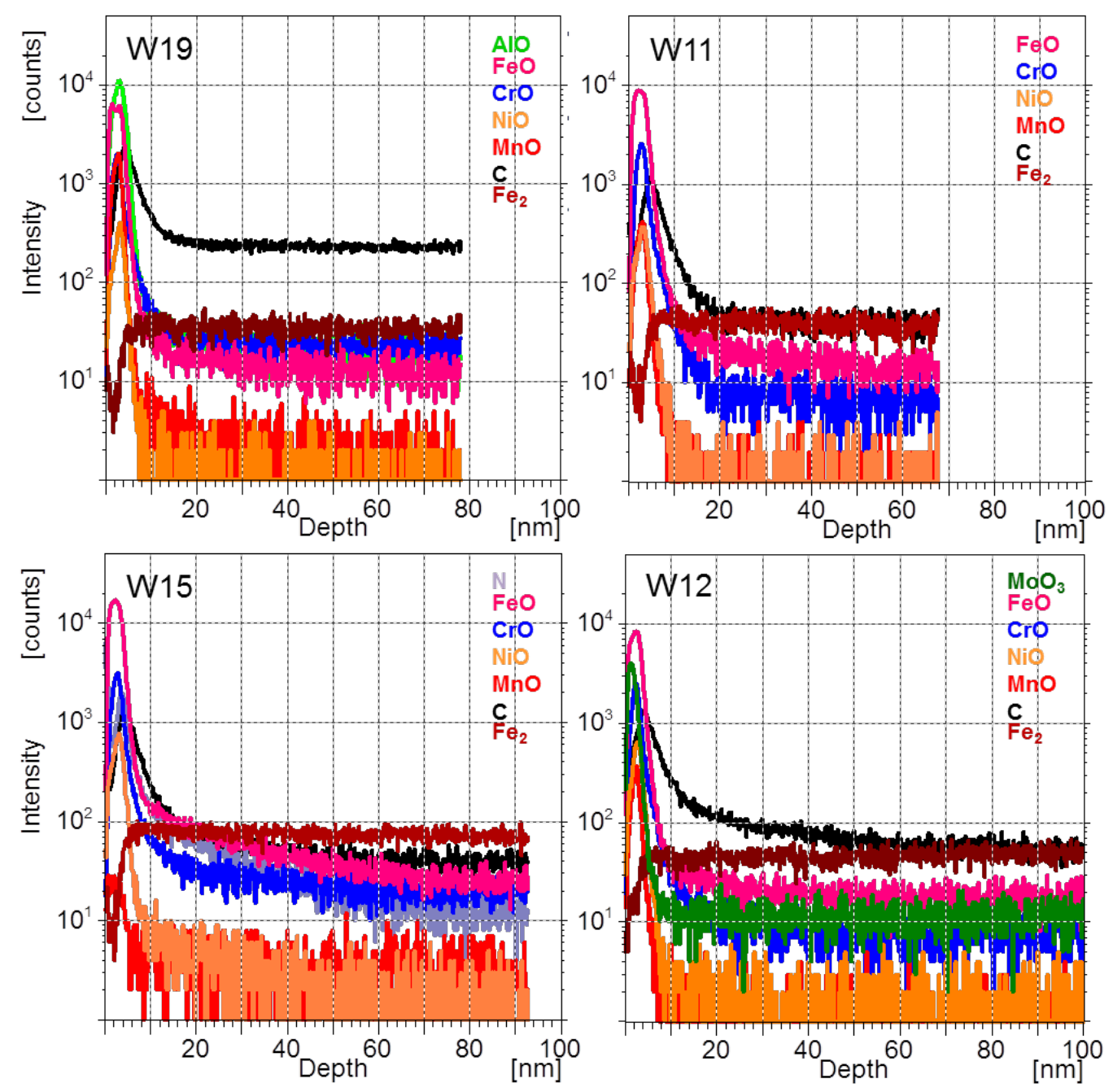

Fig. 4.26 The oxide layer formed on polished W19, W11, W15 and W12. Thin surface oxide is observed

In all cases, only a thin surface oxide layer was observed. The thickness of this oxide layer is fairly constant; $3.0-4.5 \mathrm{~nm}$ for all of these alloys. The intensity of $\mathrm{FeO}$ and $\mathrm{CrO}$ reaches the level of $\mathrm{Fe}_{2}$ after passing the thin oxide region. The intensity of $\mathrm{FeO}$ and $\mathrm{CrO}$ reaches the level of $\mathrm{Fe} 2$ after passing the thin oxide region.

A comparison of the results on the polished samples (Fig. 4.26) and the solution annealed samples (Fig. 4.21) indicates that (a) thick oxide, (b) 
decaying sub-surface oxide and (d) high constant oxide level found in Fig. 4.21 are characteristics of solution annealing, resulting from the migration of metals or oxygen ions at high temperature oxidation process. In contrast, (c) thin surface oxide is similar to that formed in polished condition. In this type of oxide layer, the only difference between polished and annealed is the thickness of the top surface oxide layer.

\subsubsection{Dislocation density measured by XRD}

From the concept of austenite stability, we expect a correlation between RRA vs Ni content of stainless steels. However, as shown in Fig. 4.20, a large scattering of these relationship was found even for alloys with greater nickel content $(\mathrm{Ni}>11 \mathrm{wt} \%)$. Further, RRA of W11 changes by solution annealing time (Fig. 4.15). From TEM observations, the difference of dislocation density and distribution was not considerable between 15-min and 30-min annealed samples (compare section 4.2.2.3). However, TEM observations often refer only to a local region. Considering these results, we performed dislocation density measurements on the low-nickel steels by using XRD, in order to gain more global information on the dislocation density of these samples.

Fig. 4.27 shows the dislocation density in the low-nickel steels plotted as a function of RRA in order to reveal the relationship between them. It should be noted that the dislocation density was measured in solution annealed samples, so that this figure indicates the influence of the dislocation density in the alloys prior to the tensile tests. The colored points represent the oxide layer type as described in 4.3.2.

From Fig. 4.27, it can be seen that the dislocation density in samples can vary by two orders of magnitude. As described in 4.2.2.3, in general, dislocation density of annealed metal is in the order of $10^{10-10^{12}} \mathrm{~m}^{-2}$ [121]. Despite of the solution annealing process, all of the samples except W11 possess a higher dislocation density than the value in literatures. 


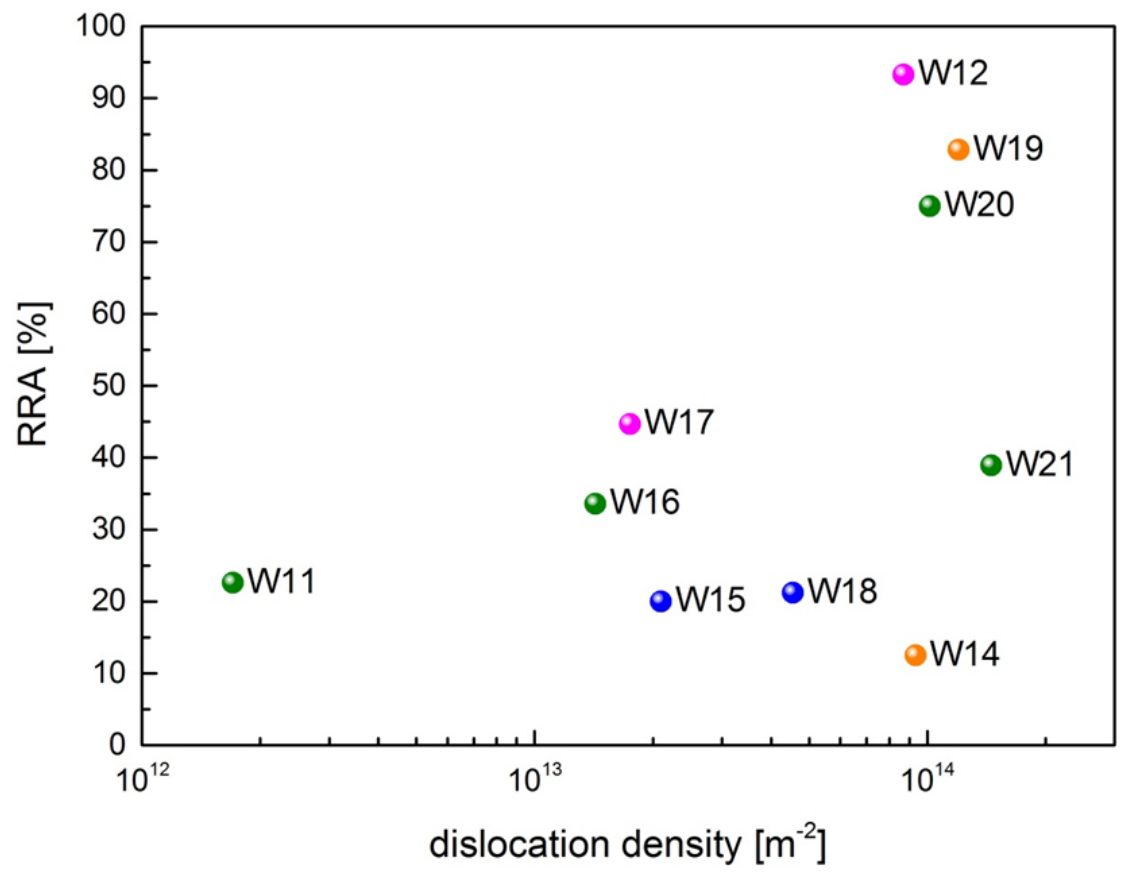

Fig. 4.27 Dislocation density in W11-W21 plotted as a function of RRA. All samples were solution annealed for $30 \mathrm{~min}$ by graphite furnace. RRA values obtained from tensile tests performed in air at $-50{ }^{\circ} \mathrm{C}$, atmospheric pressure and in hydrogen at $-50{ }^{\circ} \mathrm{C}, 40$ $\mathrm{MPa}$. The colored points represent the type of oxide layer, (a) thick oxide: orange, (b) decaying sub-surface oxide: olive, (c) thin surface oxide: blue, and (d) high constant oxide level: magenta

W12, W19 and W20, which possess a high dislocation density, show high RRA value, while W14 and W21, which also contain high dislocation density as well, though, shows low RRA value. Thus, at least in the frame of this study the dislocation density does not systematically scale with the RRA-values.

\subsubsection{Martensite formation at - $50{ }^{\circ} \mathrm{C}$ in air}

In order to evaluate the influence of the austenite stability on $\mathrm{HE}$, martensite transformation curves, which were measured during the tensile test in air at $-50{ }^{\circ} \mathrm{C}[20]$, were analyzed according to the Olson-Cohen model [123]. The volume fraction of $\alpha^{\prime}$-martensite $f^{\alpha^{\prime}}$ as a function of true strain $\varepsilon$ is expressed as (4-1),

$$
f^{\alpha^{\prime}}=1-\exp \left\{-\beta[1-\exp (-\alpha \varepsilon)]^{n}\right\}
$$


where $\alpha$ and $\beta$ are temperature dependent constants and $n$ is a fixed exponent of value 4.5 for austenitic stainless steels [123]. The martensite transformation provided in Ref. [20] was plotted as a function of engineering strain. Thus, engineering strain was converted to true strain by using Eq. (2-12). Fig. 4.28 shows martensite transformation curves as a function of true strain along with curve fits based on the Olson-Cohen model. In case of W14, the curve fitting was not carried out. The steep curve seen for W14 in Fig. 4.28 indicates that this material contains a'-martensite which transformed thermally at $-50{ }^{\circ} \mathrm{C}$ without strain [20]. For W14, therefore, the Olson-Cohen model Eq. (4-1) cannot be applied without any modification, while it can be applied for all other samples.

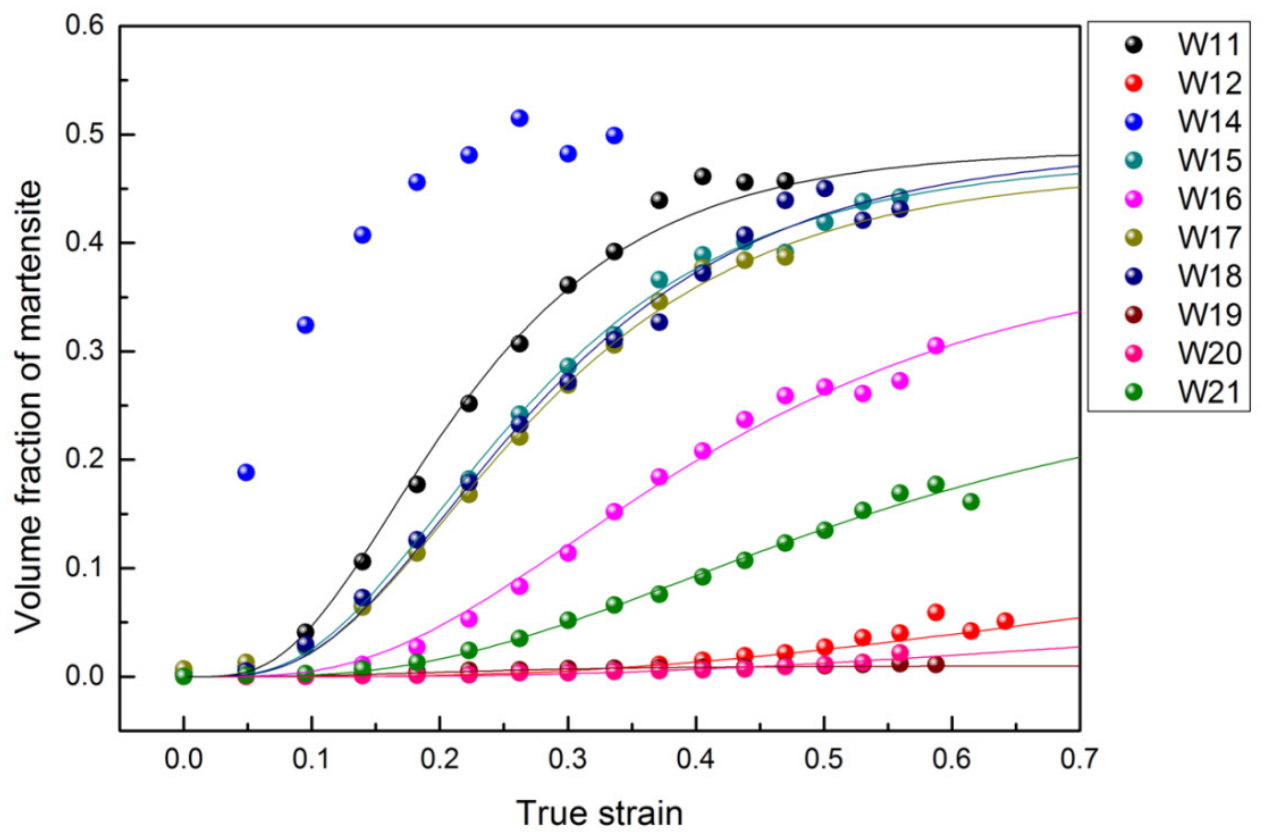

Fig. 4.28 Martensite transformation curves measured during tensile test in air at $-50^{\circ} \mathrm{C}$, at atmospheric pressure. The original data are provided by Martín [20], with permission of the author. Experimental data are indicated by points. The solid curves represent the fits using the Olson-Cohen model.

In Eq. (4-1), $\alpha$ describes the rate of shear-band formation with respect to strain. It is mainly depends on the stacking fault energy (SFE). $\beta$ is related to the probability of the formation of a'-martensite nuclei. In other words, $\beta$ gives an information about the rate of phase transformation into martensite. For larger $\beta$ values the volume fraction of the martensite phase is larger. It, 
thereby, gives the information about the sample's ability to form a martensite. As a result, the two parameters, $\alpha$ and $\beta$, are obtained by fitting the martensite transformation curve by using Eq. (4-1). As can be seen in Fig. 4.28, the fitting results are satisfying within the tested strain range.

Fig. 4.29 shows the RRA plotted as a function of (a) $\alpha$ and (b) $\beta$ on W11-W21. The colored points represent the oxide layer type as described in 4.3.2. A good linear relationship between the RRA-value and the $\beta$-parameter was found, as shown by the dashed line, for most of the alloys. A less pronounced linearity was found between the RRA-value and the $\alpha$-parameter. This result, therefore, suggests that the $\beta$-parameter, the formation probability of a'-martensite nuclei, is the predominant factor to HE.

Judging from Fig. 4.29 (b), samples with three types of oxide (a) thick oxide, (b) decaying sub-surface oxide and (c) thin surface oxide fall in the linear relationship between the RRA-value and the calculated probability of the nucleation of $\alpha^{\prime}$-martensite $(\beta)$. One can expect that the thick $\mathrm{Al}$ oxide on W19 increases the RRA-value if the oxide layer works as a protective layer. However, the tensile test measurement was carried out on W19 after removing the thick oxide layer, which was formed during the solution annealing, by polishing. Surprisingly, the RRA-value of W19 without the thick oxide is almost same as that of W19 with the thick oxide layer [20]. As a result, the thick $\mathrm{Al}$ oxide layer on W19 is not causing the additional increase of the RRA.

Martensite formation seems to play a major role to $\mathrm{HE}$ in these austenitic stainless steels. W12 and W17, both are of oxide type (d), with the high constant oxide level, do not follow the general trend. Their RRA value with respect to the $\beta$-parameter is higher than that of the related other samples. 


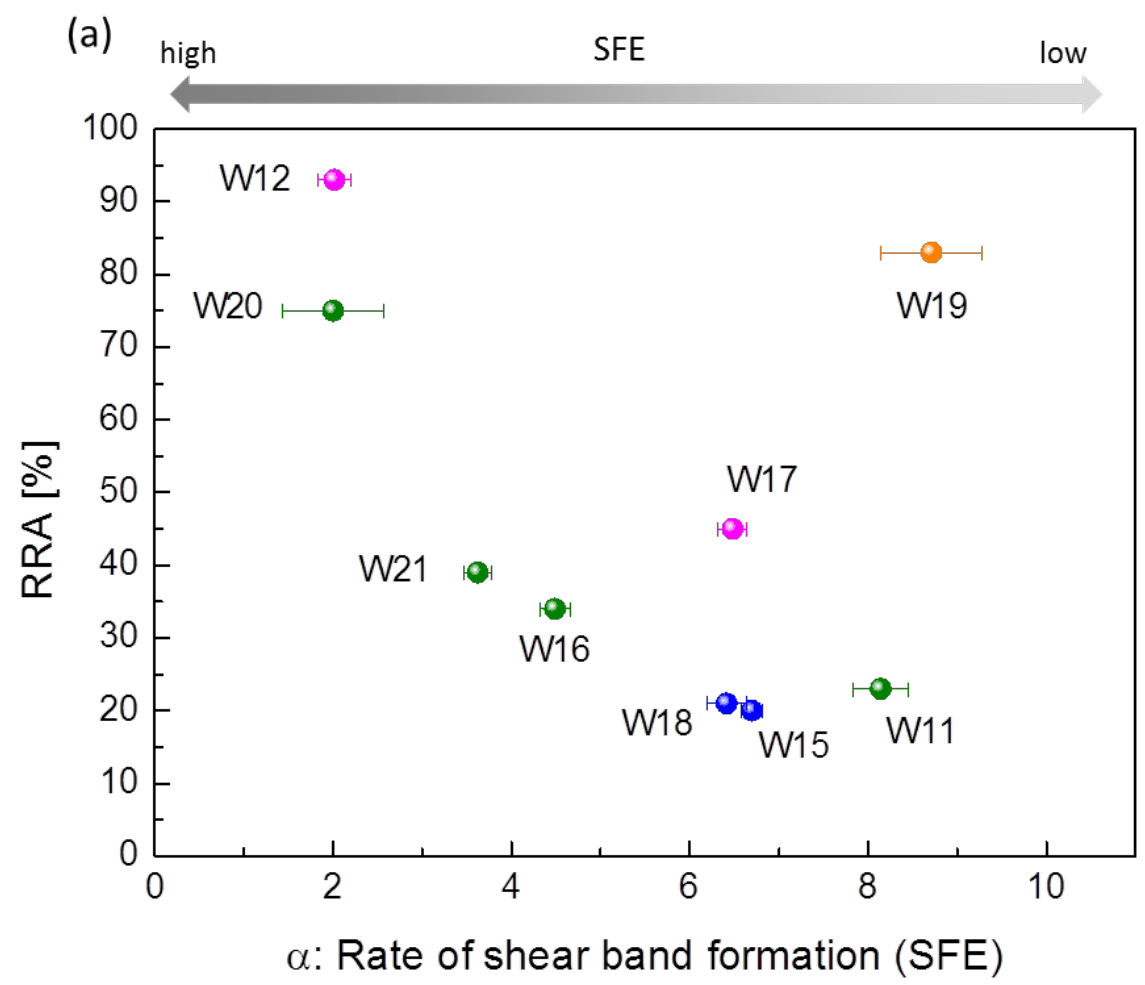

(b)

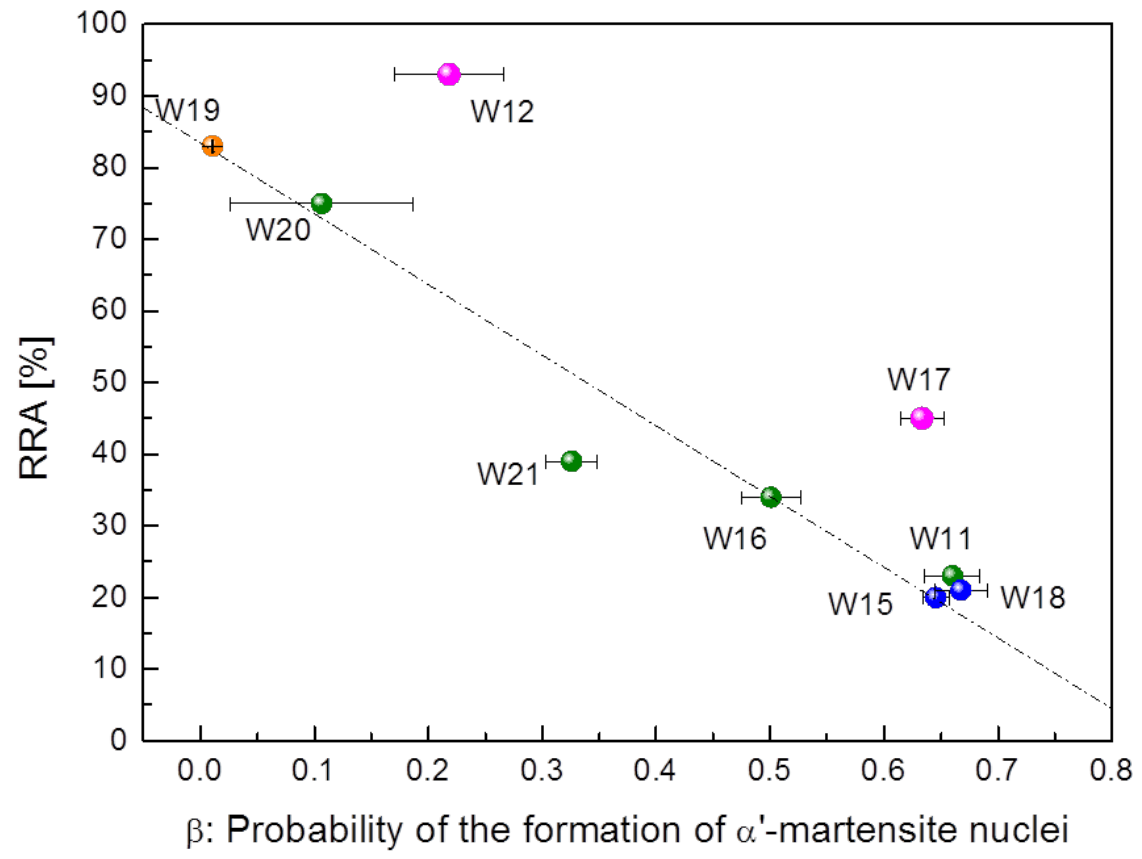

Fig. 4.29 RRA plotted as a function of $\alpha$ and $\beta$ obtained by fitting results on martensite transformation curves using Olson-Cohen model. A Linear relationship was found between the $\beta$-parameter and the RRA value. The colored points represent the type of oxide layer, (a) thick oxide: orange, (b) decaying sub-surface oxide: olive, (c) thin surface oxide: blue, and (d) high constant oxide level: magenta 


\section{Discussion}

The impact of many aspects such as oxide layer type and thickness, a'-martensite-content, dislocation density etc. on $\mathrm{HE}$ in austenitic stainless steels were studied in order to interpret the large scattering in the RRA-values on similar austenitic stainless steels with low-nickel content. In this chapter, the results obtained by different measurements are systematically discussed and regarded in the frame of present literature. Firstly, the oxide layer formation process of austenitic stainless steels is discussed. Understanding the oxide layer formation is of considerable importance because the hydrogen entry in metals is highly sensitive to the surface. After that, the discussion treats the impact of the a'-martensite on $\mathrm{HE}$, since $\mathrm{HE}$ is predominantly governed by the diffusion in martensite phase. Then, the influence of microstructure (grain size, dislocation density and dislocation structures) is specified in order to reveal the source of scattering in RRA. Further, the effect of microstructures is taken into consideration. Finally, the discussion is summarized by focusing on the impact of the oxide layer on HE.

\subsection{Oxide layer formation}

In this study, the solution annealing was conducted at $1050^{\circ} \mathrm{C}$ or $1150{ }^{\circ} \mathrm{C}$ depending on the chemical composition of samples. As shown in Fig. 4.2, the oxide layer thickness strongly depends on the gaseous environment in the furnace. For example, the thin oxide layer formed in graphite heater is due to the reduction atmosphere in the furnace. The oxide layer stacking was found for all samples except for W14 and W19. These samples, W14 and W19, have high aluminum concentration and their oxide layer was characterized as a thick oxide type. (Fig. 4.21). Here, we note that the difference in the gaseous environment only affects the oxide layer thickness, but not the layer stacking sequence, as can be seen in Fig. 4.2. In the following section, the oxide layer sequence and oxide layer formation will be discussed separately for the alloying elements. 


\subsubsection{Iron (Fe), Chromium (Cr), Nickel ( $\mathrm{Ni})$}

\subsubsection{Stacking sequence of oxide}

The depth profiles of oxide layer formed at high temperature reveal a typical oxide layer stacking of $\mathrm{Fe}^{-}, \mathrm{Cr}^{-}$and $\mathrm{Ni}$-oxide (Fig. 4.2, Fig. 4.3, Fig. 4.6, Fig. 4.12, Fig. 4.16, Fig. 4.21). The layer sequence is as follows: The outer layer is a Fe-rich oxide while the inner layer is a mixed $\mathrm{Fe}-\mathrm{Cr}-\mathrm{Ni}$ oxide. The maximum peak of $\mathrm{Ni}$ locates beneath the maximum peak of $\mathrm{Cr}^{-o x i d e}$. This sequence is clearly confirmed in Fig. 4.2, Fig. 4.3, Fig. 4.6, Fig. 4.12.

The oxide layer stacking in AISI 300 series stainless steels has been reported by a number of authors using various surface analysis techniques such as X-ray Photoelectron Spectroscopy (XPS), Auger electron spectroscopy (AES) and Secondary Ion Mass Spectrometry (SIMS) [124-135]. In case of air oxidation at low temperatures (up to $400{ }^{\circ} \mathrm{C}$ ), their results show that the outer layer is $\mathrm{Fe}$ rich oxide and, the inner layer is a $\mathrm{Cr}$ oxide $[124,130,132,134,135]$. At temperatures above $400{ }^{\circ} \mathrm{C}$, there is a controversy concerning the stacking sequence of the oxide layer. Betz et al. studied oxide layers on AISI 304 by AES and found an Fe-rich outer oxide layer when oxidized below $500{ }^{\circ} \mathrm{C}$ [124]. At $700{ }^{\circ} \mathrm{C}$, Cr was detected in the outer layer and the $\mathrm{Cr}$ concentration in the outer layer becomes predominant with elevating oxidation temperatures, of up to $900{ }^{\circ} \mathrm{C}$. In contrast, some researchers reported the formation of an Fe-rich outer layer at above $400{ }^{\circ} \mathrm{C}$ [126,133]. Recently, Vesel et al. have reported the depth profiles on AISI 316L by AES [135]. The oxidation was conducted in the temperature range between $327{ }^{\circ} \mathrm{C}$ to $977^{\circ} \mathrm{C}$. At temperatures below $327^{\circ} \mathrm{C}$, the outer oxide layer consists of $\mathrm{Fe}$, while $\mathrm{Cr}$ concentration is maximum at the oxide-bulk interface. At $527{ }^{\circ} \mathrm{C}$, chromium oxide becomes dominant and Fe signal significantly decreases in the outer oxide layer. However, at $727^{\circ} \mathrm{C}$, the $\mathrm{Fe}$ signal is, again, enriched at the surface. Further increase in oxidation temperature $\left(980{ }^{\circ} \mathrm{C}\right)$ leads to predominant Fe in the outer oxide layer.

The discrepancy of the oxide layer sequence shown above may be due to the difference in the thickness of the surface layer analyzed by AES. Typical 
information depth (electron escape depth) for AES is several nm [104], resulting in the difficulty to detect a thin layer $(<$ several $\mathrm{nm}$ ). In case of SIMS, the several $\mathrm{nm}$ of Fe-rich outer layer can be detected, as shown in Fig. 4.21 because the information depth (ion escape depth) for SIMS is one monolayer to a few layers. Moreover, the intensity is used in SIMS to represent the depth profile while the atomic fraction is typically used in AES and XPS. As a consequence, the presence of Fe in the outer layer could be obscured in AES and XPS results, but not in SIMS results.

\subsubsection{Oxide type}

In contrast to AES and XPS, SIMS measurement does not give information about the chemical state of oxides. In order to determine the oxide type, the two dimensional phase stability diagram for the $\mathrm{Fe}^{-} \mathrm{Cr}-\mathrm{O}$ system can be applied. Fig. 5.1 shows the phase stability diagram for the $\mathrm{Fe}-\mathrm{Cr}-\mathrm{O}$ and $\mathrm{Fe}-\mathrm{Ni}-\mathrm{O}$ system at $900{ }^{\circ} \mathrm{C}$ versus the oxygen partial pressure [136]. The compositional range of stainless steels used in this study is roughly indicated with a red dotted rectangle in Fig. 5.1.

When a metal reacts with oxygen, a gradient of the oxygen chemical potential occurs from the surface to the bulk. Thus, the oxygen partial pressure of the stability diagram can be assumed as the gradient of oxygen chemical potential in a metal since the oxygen partial pressure decreases with depth. Comparing Fig. 4.21 and Fig. 5.1, it is likely that the outer oxide layer is a corundum type $\mathrm{Fe}_{2} \mathrm{O}_{3}$. In the deeper region of oxide it could be a mixture of corundum $\left(\mathrm{Fe}_{2} \mathrm{O}_{3}, \mathrm{Cr}_{2} \mathrm{O}_{3}\right)$ and spinel $\left(\mathrm{FeCr}_{2} \mathrm{O}_{4}, \mathrm{NiFe}_{2} \mathrm{O}_{4}\right.$ and $\mathrm{NiCr}_{2} \mathrm{O}_{4}$ ) type. The oxide type in the deeper region is unclear. Regarding the composition of the outer layer, Shibagaki [133] and Saeki [137] investigated the oxide layer formed on AISI 300 series stainless steels at $427^{\circ} \mathrm{C}$ and $1000{ }^{\circ} \mathrm{C}$, respectively. Shibagaki et al. evaluated the thin oxide layer consisting of five layers by comparing the chemical potential diagrams with the depth profile results. They concluded that the outer layer (first layer) is corundum-type $\mathrm{Fe}_{2} \mathrm{O}_{3}$ and a spinel-type $\mathrm{NiFe}_{2} \mathrm{O}_{4}$ and the interface (fifth layer) is spinel-type compound $\mathrm{FeCr}_{2} \mathrm{O}_{4}$, coexists with a solid solution of $\mathrm{Ni}$ 
Corundum: $\mathrm{A}_{2} \mathrm{O}_{3}$,Spinel: $\mathrm{A}_{3} \mathrm{O}_{4}$

$(\mathrm{A}=\mathrm{Fe}, \mathrm{Ni}, \mathrm{Cr})$

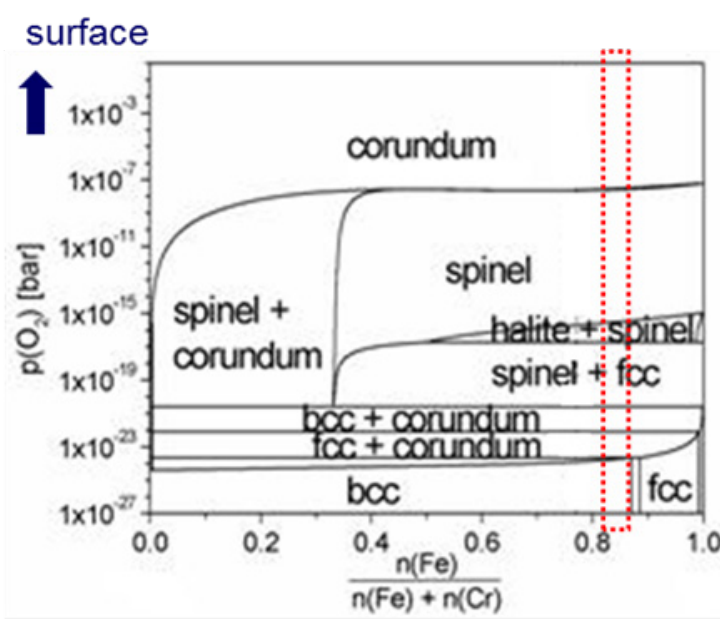

(a) $\mathrm{Fe}-\mathrm{Cr}-\mathrm{O}$

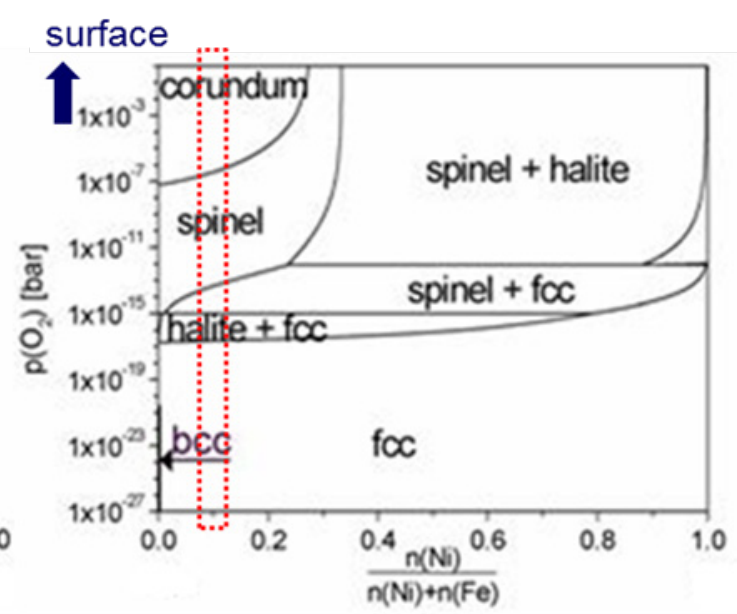

(b) Fe-Ni-O

Fig. 5.1 Phase stability diagram at $900{ }^{\circ} \mathrm{C}$ (a) Fe-Cr-O and (b) Fe-Ni-O system [136]. Red dotted rectangle indicates the compositional region used in this study. (Figure from Hansson [136])

and Fe [133]. In contrast, Saeki et al. investigated thin oxide layers by XPS and XRD and observed that the outer layer is a mixed layer of corundum and spinel type oxides, and the inner layer is a chromium-rich corundum type [137]. Some researchers studied thin oxide layers formed at over $1000{ }^{\circ} \mathrm{C}$ [126,137]. Thus, more detailed experimental research on the thin oxide layer under certain harmonized conditions is required to get the full picture. Especially at high temperatures like $1000{ }^{\circ} \mathrm{C}$, considerable degassing is expected and thus the partial pressure of various gases in the furnace should give rise to different oxidation conditions.

The oxidation process is schematically illustrated in Fig. 5.2, as is often used to describe the oxide layer formations [126,138-142]. In order to simplify the model, only the migration of metal ions from bulk is presented by arrows, although the transport of oxygen from the surface toward the bulk also occurs during oxidation. 


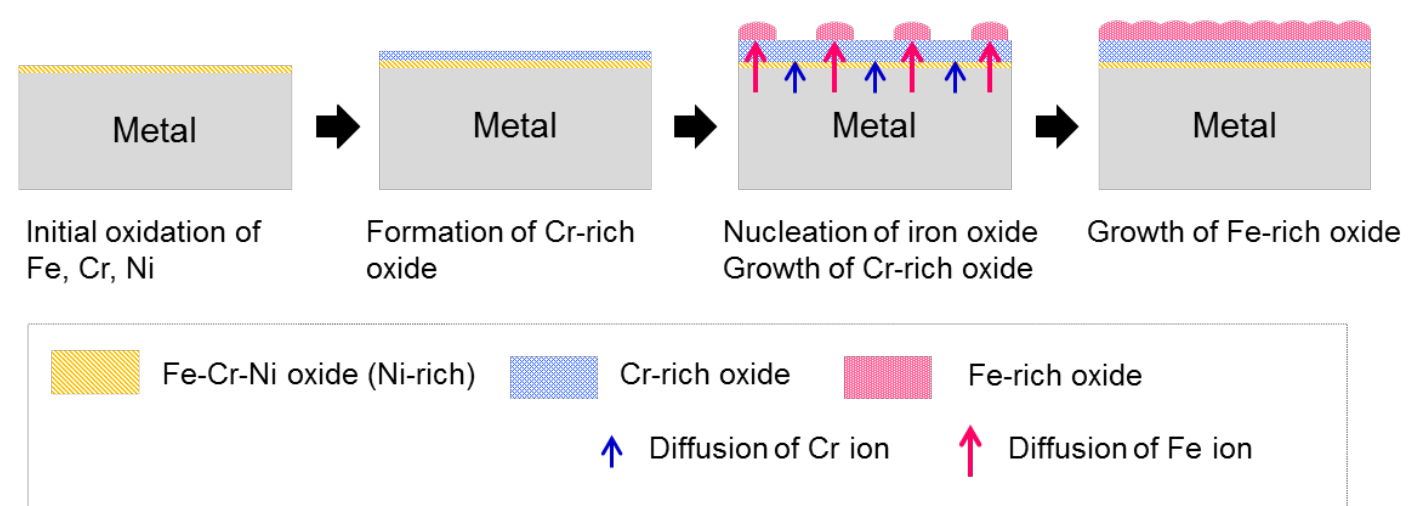

Fig. 5.2 Schematic model of the oxidation process for the steels in this study

In the initial stage of oxidation, $\mathrm{Fe}, \mathrm{Cr}$ and $\mathrm{Ni}$ react with oxygen at the surface and a mixture of $\mathrm{Fe}-\mathrm{Cr}-\mathrm{Ni}$ is formed on the alloy surface. When the oxidation proceeds, $\mathrm{Cr}$ is predominantly oxidized because $\mathrm{Cr}$ oxide has the most negative free energy of formation, as shown in Ellingham diagram (Fig. 2.16). Upon further oxidation, the Fe ions diffuse through the $\mathrm{Fe}-\mathrm{Cr}-\mathrm{Ni}$ oxide and the Cr-rich oxide, resulting in the nucleation of Fe-oxide at the surface because the diffusion of $\mathrm{Fe}$ ions in the oxide is 2.5 orders of magnitude faster than that of $\mathrm{Cr}$ ions [143]. Once the Fe-rich oxide nucleates, it grows and spreads over the Cr-rich oxide. The detail of ion-diffusion in oxide will be discussed in section 5.1.5.

\subsubsection{Manganese (Mn)}

As shown in Fig. 4.21, the profile of $\mathrm{MnO}$ differs among the samples and it seems that the profile shape is associated with the type of oxide. (classification of oxide, see in section 4.2.2.2) In W11, W20 and W21, the maximum peak of $\mathrm{MnO}$ appears in the Cr-rich oxide layer (see Fig. 4.21 (b)). A considerably broad profile was found in W16. The oxide layer formed on these alloys, W11 W16, W20 and W21, is categorized as "decaying sub-surface oxide" type (Fig. 4.21 (b)). MnO distributes homogeneously in the "high constant oxide level" (W12, W17, Fig. 4.21 (d)). W15 and W18, which are categorized as thin oxide, contains rather small amount of Mn (Fig. 4.21 (c)). Thus $\mathrm{MnO}$ was detected only in the surface region. Although the reason for the difference in the distribution of $\mathrm{MnO}$ is not clear. $\mathrm{Mn}$ tends to be 
present in the surface region as shown in Fig. 4.21. The trend, at least, is not in contradiction to the results reported by Vesel, suggesting that the addition of even small quantities of $\mathrm{Mn}$ into an alloy leads to the formation of $\mathrm{MnO}$, at high temperatures [135].

\subsubsection{Carbon (C)}

A maximum peak of $\mathrm{C}$ always appears at the oxide/bulk interface in both annealed and polished samples (Fig. 4.21 and Fig. 4.26). Some researchers have monitored $\mathrm{C}$ in steels, but, most of them have attributed that $\mathrm{C}$ as contamination at the surface [144,145], constant level throughout the experiments [132,146], and close to the detection limit [147]. Only Betz et. al and Vesel et. al showed the existence of a small $\mathrm{C}$ peak at the interface in depth profiles by AES [124,134]. The presence of $\mathrm{C}$ at the oxide/bulk interface can be due to the fast interstitial diffusion of $\mathrm{C}$. The diffusion coefficient of carbon is about 4 orders of magnitude larger than that of chromium [115]. Here, we suggest that, during the oxidation process, C instantaneously diffused toward the fresh surface from the bulk in order to achieve the equilibrium state.

\subsubsection{Aluminum (AI)}

W14 and W19 have thick oxides of Al, as shown in Fig. 4.21. The oxide layer was grown to at least for $150 \mathrm{~nm}$ in W19, and for $900 \mathrm{~nm}$ in W14. The oxide layer thickness of such extent is considerably thick for SIMS depth profiles in a reasonable analysis time scale and, therefore, the depth profiles on both W14 and W19 are not showing any interface between oxide layer and bulk region, this interface is not reached in the measurements. The origin of this thick Al-oxide formation can be explained by the preferential oxidation of $\mathrm{Al}$, since $\mathrm{Al}-$ oxide is more thermodynamically stable than $\mathrm{Fe}^{-}, \mathrm{Cr}^{-}$, and $\mathrm{Ni}$-oxide as indicated by Ellingham diagram in Fig. 2.16. Taking an advantage of thse selective oxidation of $\mathrm{Al}$, the formation of $\mathrm{Al}$-oxide on alloys, so called $\mathrm{Al}_{2} \mathrm{O}_{3}$-forming (Calorizing or Aluminizing), has been widely used 
intentianly in metallurgical engineering in order to enhance e.g. the high temperature corrosion resistance of alloys [122,148-154].

Interestingly, the oxide layer of W14 was much thicker than that of W19, although the Al content of W14 (1.64 wt\%) is lower than that of W19 (2.87 $\mathrm{wt} \%$ ). This trend might be explained by the oxidation rate of $\mathrm{Al}$ depending on the $\mathrm{Al}$-content. Ike et. al has studied high temperature oxidation behavior of $\mathrm{Fe}-14 \mathrm{Cr}$ alloys with low $\mathrm{Al}$ contents [149]. In their study, the oxidation rate decreased with increasing $\mathrm{Al}$ addition from 0.75 to $5.0 \mathrm{wt} \%$. This is in accordance with our findings.

The distinct localization of $\mathrm{FeO}, \mathrm{CrO}, \mathrm{NiO}, \mathrm{MnO}$ and $\mathrm{C}$ was found in $\mathrm{W} 19$ (Fig. 4.23 and Fig. 4.24), implying the formation of multiple phases, depending on the $\mathrm{Al}$ content in alloys. According to Al-Fe phase diagram, Fe is only slightly soluble in fcc- $\mathrm{Al}$ [155]. In the $\mathrm{Al}_{2} \mathrm{O}_{3}-\mathrm{FeO}-\mathrm{Fe}_{2} \mathrm{O}_{3}$ ternary system, $\mathrm{Al}_{2} \mathrm{O}_{3}$ can dissolve up to 12 wt $\% \mathrm{Fe}_{2} \mathrm{O}_{3}$ in the temperature range $1300-1370{ }^{\circ} \mathrm{C}[156]$. When oxidation of austenitic stainless steels containing $\mathrm{Al}$ occurs, $\mathrm{Al}$ is selectively oxidized as described above. The small solubility of $\mathrm{Fe}$ in $\mathrm{Al}$ and $\mathrm{Fe}_{2} \mathrm{O}_{3}$ in $\mathrm{Al}_{2} \mathrm{O}_{3}$ results in phase separation in the oxide layer of W19 under solution annealing treatment.

\subsubsection{Kinetics of oxidation: details on ion mobilities}

As mentioned above, a stacking of different oxide layers was observed on stainless steels. If the oxidation process is thermodynamically controlled, the oxide layer would be stacked in the sequence of getting the lowest standard free energy of formation of oxides under thermodynamically equilibrium conditions, as shown in the Ellingham diagram (Fig. 2.16). However, this study, like certain others, shows that the oxide stacking sequence does not follow the order of the standard free energy formation of oxides. It means that the oxidation process is not only thermodynamically but also kinetically controlled.

To interpret the ion-diffusion rate in the oxide, as presented in section 2.3.4, the crystal field theory can be introduced [132,157]. Possible diffusion paths for cations in close-packed anion lattices and the preferred route in 
cubic structures are shown to be by alternate, adjacent octahedral and tetrahedral positions, while diffusion via similar interstices, i.e. tetrahedral to tetrahedral or octahedral to octahedral, is prohibited by the severe lattice distortion. [132]. The rate of lattice diffusion of a cation through oxide is related to the activation energy for transfer from octahedral to tetrahedral site, and vice versa. Each site has certain stabilization energy for the cations. As a consequence, the difference between the octahedral and tetrahedral crystal field stabilization energies, termed the crystal field preference energy (CFPE), implies the relative ion mobility in the oxide. The crystal field stabilization energies [158] and CFPE [132] for transition-metals in spinels are listed in Table 5.1 with their ionic radii [24].

In Table 5.1, we should note that the results are given for a certain oxidation number of the cations. Any change in the oxidation state may change the values. According to the summary in Table 5.1, both $\mathrm{Cr}^{3+}$ and $\mathrm{Ni}^{2+}$ prefer octahedral sites. In contrast, $\mathrm{Fe}^{3+}$ and $\mathrm{Mn}^{2+}$ have no site preference. It suggests that once $\mathrm{Cr}^{3+}, \mathrm{Ni}^{2+}$ and $\mathrm{Fe}^{2+}$ do not easily move to the tetrahedral site through an adjacent octahedral site, while $\mathrm{Fe}^{3+}$ and $\mathrm{Mn}^{2+}$ migrate through the route consisting of alternate tetrahedral and octahedral sites. This model explains the fast diffusion of $\mathrm{Mn}^{2+}$ despite of its relatively large ionic radius compared to that of $\mathrm{Cr}^{2+}$.

Table 5.1 Crystal field stabilization [158], Crystal field Preference Energies (CFPE) [132] for transitionmetal ions in oxides and ionic radii [24]. The CFPE is defined as the difference between the octahedral and tetrahedral crystal field stabilization energies of oxide. (Data from Dunitz [158], Allen [132] and Hasegawa [24])

\begin{tabular}{cccccc}
\hline Ion & $\begin{array}{c}\text { Number of d } \\
\text { electrons }\end{array}$ & $\begin{array}{c}\text { Octahedral } \\
\text { Stabilization } \\
(\mathrm{kJ} / \mathrm{mol})\end{array}$ & $\begin{array}{c}\text { Tetrahedral } \\
\text { Stabilization } \\
(\mathrm{kJ} / \mathrm{mol})\end{array}$ & $\begin{array}{c}\text { CFPE } \\
(\mathrm{kJ} / \mathrm{mol})\end{array}$ & $\begin{array}{c}\text { lonic radii } \\
(\mathrm{nm})\end{array}$ \\
\hline $\mathrm{Cr}^{3+}$ & 3 & 224.7 & 66.9 & 158 & 0.063 \\
$\mathrm{Mn}^{2+}$ & 5 & 0 & 0 & 0 & 0.082 \\
$\mathrm{Fe}^{3+}$ & 5 & 0 & 0 & 0 & 0.065 \\
$\mathrm{Fe}^{2+}$ & 6 & 49.8 & 33.1 & 17 & 0.079 \\
$\mathrm{Co}^{2+}$ & 7 & 92.9 & 61.9 & 31 & 0.077 \\
$\mathrm{Ni}^{2+}$ & 8 & 122.2 & 36.0 & 86 & 0.073 \\
\hline
\end{tabular}


On this basis, the ranking order of relative ion mobility in the oxide grown on a stainless steel might be as follows:

$$
\mathrm{Fe}^{3+} \text { and } \mathrm{Mn}^{2+}>\mathrm{Fe}^{2+}>\mathrm{Co}^{2+}>\mathrm{Ni}^{2+}>\mathrm{Cr}^{3+}
$$

The order of ion mobilities agrees well with the stacking sequence of $\mathrm{Fe}^{-}$ and Mn-oxide determined by this SIMS study. But, the stacking sequence of $\mathrm{Cr}^{-}$and $\mathrm{Ni}^{-}$oxide differs from the expected order. In the SIMS results, the peaks in the $\mathrm{NiO}$ curves were found beneath the CrO. This is not consistent with the order of ion mobilities noted above. Similar trends to this SIMS observation have been reported by several authors [124,128-130,133,134]. The reason of this behavior could be that large amount of Ni being oxidized at the beginning of oxidation. According to the Ellingham diagram (Fig. 2.16), the standard free energy of the formation of $\mathrm{Ni}$ oxide is positive than that of Cr oxide, suggesting lower affinity between oxygen and Ni. However, at the beginning of oxidation, the partial pressure of oxygen is so high enough at the surface, that $\mathrm{Ni}$ could be predominantly oxidized at this stage of the whole reaction processes due to its high ion mobility. Assuming that Ni oxide with several monolayers is formed at the surface, a gradient of oxygen chemical potential from the surface-side to the bulk occurs, then leading to a selective oxidation of $\mathrm{Cr}$. $\mathrm{NiO}$ being buried under the $\mathrm{Cr}$ oxide layer. Therefore the maximum peak of $\mathrm{CrO}$ is slightly shallower than the NiO peak. At later stages, differences in diffusion rates control the formation of oxides. The oxidation of $\mathrm{Fe}$ is thermodynamically unfavorable. However, its greater mobility allows diffusion through the Ni-rich oxide and $\mathrm{Cr}$-rich oxide and the formation of Fe-rich oxides at the outer layer should result. The same should account for the oxidation of $\mathrm{Mn}$.

\subsection{Hydrogen embrittlement}

\subsubsection{Influence of surface strain-induced a'-martensite and oxide layer}

In order to reveal whether strain-induced a'-martensite or the oxide layer plays a major role in the RRA-value, tensile tests and SIMS analysis on 
W11 with and without a'-martensite were performed, as shown in section 4.2.1. W11 with a'-martensite is in the as-machined condition since austenite phase $(\mathrm{Y})$ partially transforms to a'-martensite during the machining process. Under the machining condition, $50 \mu \mathrm{m}$ thick a'-martensite was formed at the surface as shown in Fig. 4.9. The a'-martensite-free W11 was produced by solution annealing at $1050{ }^{\circ} \mathrm{C}$ for $15 \mathrm{~min}$. The oxide layer type formed on W11 without a'-martensite is "high constant oxide level", as described in section 4.2.2.2.

The RRA-value of W11 with a'-martensite and a layer of high constant oxide level was as low as $51.6 \%$ compared to $84.1 \%$ for W11 without a'-martensite solution annealed (decaying sub-surface oxide layer), as summarized in Table 4.1. This means, W11 with a'-martensite was less ductile than W11 without a'-martensite when tested in hydrogen gas. Consequently, W11 with a'-martensite was more susceptible to HE than W11 without $\alpha$-martensite.

The layer of high constant oxide level formed on W11 without a'-martensite should inhibit hydrogen entry. However, during the tensile test the surface oxide breaks and crack growth occurs, suggesting that the oxide layer only initially prevents the hydrogen attacks. As a consequence, a'-martensite is judged to play a major role in the HE process. In addition, this, in turn, suggests a considerable impact of the HE- testing method itself to evaluate a HE-susceptibility. This point will be discussed in detail in section 5.2.3.3.2.

The susceptiblility of W11 with a'-martensite to HE is mainly related to the presence of the martensite phase at the sample surface. Hydrogen diffusivity in a'-martensite (bcc) is about three orders of magnitude higher than in austenite phase (fcc) [16]. During tensile tests in $40 \mathrm{MPa}$ hydrogen atmosphere, hydrogen penetrates into the specimen via the surface a'-martensite, which acts as a "hydrogen diffusion highway" [159]. Hydrogen atoms in the specimen then solute in the austenite (fcc) phase where the solubility for hydrogen is high or traps at internal defects. 


\subsubsection{Influence of austenite stability}

As discussed in the previous section, the surface $\alpha^{\prime}$-martensite acts as a rapid diffusion path for hydrogen. During tensile tests, it is also expected that the austenite phase partially transforms to a'-martensite by deformation process. The amount of a'-martensite, formed during tensile tests, depends on the strain rate, the tensile test temperature, and the chemical composition of the stainless steels. In case of commercial stainless steels such as AISI 304 and 316, many researchers have reported the effect of the tensile test temperature and the strain rate on the martensite transformation behavior [35,36,123,160-163]. Further, influences of a'-martensite on tensile test properties in hydrogen atmosphere under various test conditions are also reported. Details on these aspects are already described in sections 2.2.3.3 and 2.2.3.4. This section focuses on impacts of the chemical composition on the martensite transformation.

To eliminate the effect of pre-existing surface $\alpha$-martensite, all steels (W11-W21) were solution annealed at 1050 or $1150{ }^{\circ} \mathrm{C}$ for $15 \mathrm{~min}$ before testing. All of the tensile tests were performed at constant temperature $-50{ }^{\circ} \mathrm{C}$ and at a constant strain rate of $5.5 \times 10^{-5} \mathrm{~s}^{-1}$. At this test temperature, a'-martensite transformation more easily occurs than at room temperature.

As described already, the presence of a'-martensite increases the hydrogen susceptibility of steels. From this viewpoint, it is readily expected that the susceptibility to HE has a correlation to the steels' phase stability. The Ni content is often used as an indicator of austenite stability, since $\mathrm{Ni}$ is a strong austenite stabilizer. Several papers reported clear correlation between the RRA-values and the $\mathrm{Ni}$ content [164,165]. However, as previously mentioned (chapter 1 and section 4.2.2.1), a poor correlation between the RRA-values vs the $\mathrm{Ni}$ content is found as well, in the range of less than $10 \mathrm{wt} \% \mathrm{Ni}[18]$. In this study, large scattering was found even for the alloys with greater $\mathrm{Ni}$ contents $(\mathrm{Ni} \sim 11 \mathrm{wt} \%)$ as shown in Fig. 4.20. These facts suggest that the $\mathrm{Ni}$ content does not provide enough accuracy to estimate the austenite stability of low-nickel steels.

In this study, we found a strong correlation between the RRA-value and 
the probability of the formation of a'-martensite nuclei (B), as shown in Fig. 4.29. Here, the probability of a' nuclei formation is obtained from Olson-Cohen model. In the following sections, firstly, the Olson-Cohen model will be interpreted. Then, the findings in Fig. 4.29 will be discussed. Finally, three alternative empirical equations will be examined based on the relationship between the RRA-value and so-called B-parameter.

\subsubsection{Olson-Cohen model: details on $\mathrm{a}-$ and $\beta$-parameters}

As described in section 4.3.4, the Olson-Cohen model gives an information about the rate of shear band formation ( $\alpha$-parameter) and the probability of the formation of a'-martensite nuclei (B-parameter). The former is related to the stacking fault energy (SFE) and the latter corresponds to the austenite stability $[166,167]$.

Before discussing the dependency of $\alpha^{-}$and 6 -parameters on RRA, the content of these parameters are described in detail. The nucleation of a'-martensite is schematically illustrated by Olson and Cohen [166], based on the works of Bogers and Burgers [168], as shown in Fig. 5.3. According to Bogers and Burgers [168], an a'-martensite (bcc) structure can be generated from an austenite (fcc) structure by two successive shears. These shears are $1 / 3$ and $1 / 2$ fcc twinning shear, referred to as $\frac{T}{2}$ and $\frac{T}{3}$ in Fig. 5.3. In fcc metals, it is known that a perfect dislocation of $\frac{a}{2}\langle 110\rangle$ dissociates in two partial dislocations as described in Eq. (5-1).

$$
\frac{a}{2}<110>\rightarrow \frac{a}{6}<211>+\frac{a}{6}<12 \overline{1}>
$$

The $\frac{T}{2}$ shear can be accomplished by an array of $\frac{a_{f c c}}{6}[21 \overline{1}]$ Shockley partial dislocations on every second $(1 \overline{1} 1)_{f c c}$ plane, while the $\frac{T}{3}$ shear can be achieved by an array of $\frac{a_{f c c}}{6}[211]$ Shockley partial dislocations on every 
third $(\overline{1} 11)_{f c c}$ plane, shown in Fig. 5.3 (a). After intersection of these two arrays, the stacking faults left behind, resulting in an $\varepsilon^{-m a r t e n s i t e ~(h c p) ~}$ platelet structure, as shown in Fig. 5.3 (b). The $\varepsilon^{-}$martensite platelet extends in a band structure during the deformation. Further, an a'-martensite (bcc) nucleus is formed at the intersections between the $\varepsilon$-martensite bands.

In Fig. 5.3, $\varepsilon$-martensite is shown as an intermediate phase in the transformation from austenite to a'-martensite. However, a'-martensite transformation can also proceed without going through the $\varepsilon$ phase. The a'-martensite nucleates at slip band intersections from the austenite phase with the aid of pile up dislocations [161,169-171].

(a) before intersection process

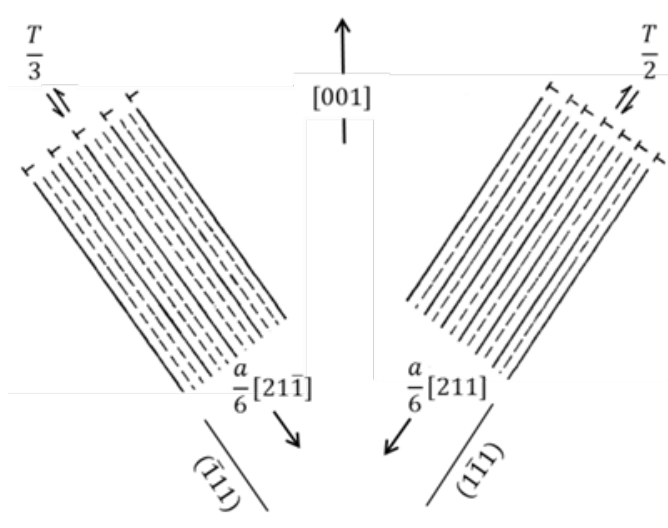

(b) after intersection process

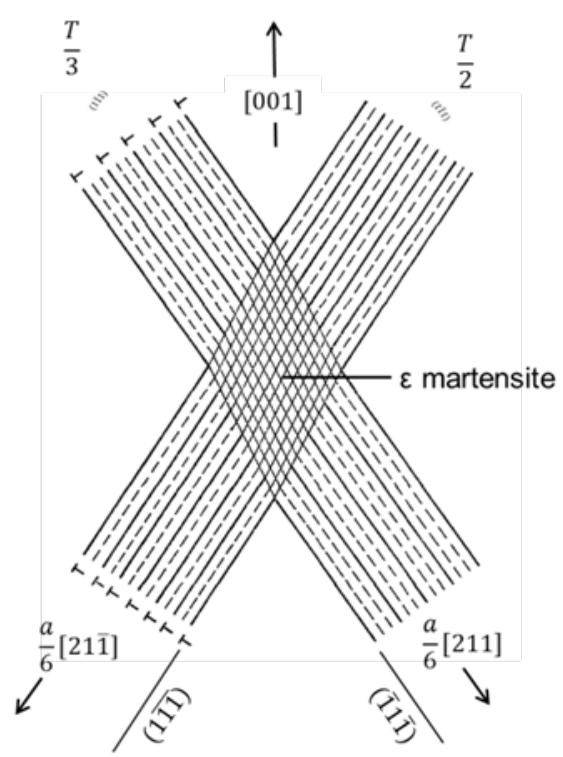

Fig. 5.3 Schematic illustration of the nucleation of $\alpha^{\prime}$ martensite at the shear band intersection due to two arrays of $a_{f c c} / 6\langle 112\rangle$ Schockley partial dislocations in austenite. (a) before intersection process and (b) after intersection process. One array $T / 2$ has partial dislocations on every second $\{111\}_{f c c}$ plane and averages one-half of a twinning shear, while the other array $T / 3$ has partial dislocations on every third $\{111\}_{f c c}$ plane and averages one-third of a twinning shear. The resulting doubly-faulted intersection has a hcp structure $(\varepsilon$-martensite) $[166,167]$. (Picture from Bracke[167], modified) 
Thus, the $\alpha$-parameter in the Olson-Cohen model corresponds the rate of shear band formation via stacking faults represented as $\frac{T}{2}$ shear, $\frac{T}{3}$ shear and $\varepsilon$-martensite. The 8 -parameter corresponds to the probability of the formation of a'-martensite nuclei formed at shear band intersections.

\subsubsection{Stacking fault energy and Martensite transformation}

A controversy exists concerning HE of austenitic stainless steels. Some authors argued strain-induced a'-martensite playing a major role in $\mathrm{HE}$ since martensite phase (bcc), formed during tensile test, acts as "hydrogen diffusion highway" $[13,15,16,159,164,172,173]$. Other authors propose HE is correlated to low stacking fault energy (SFE) [165,174-177], since hydrogen-assisted fracture has been observed for stable austenitic stainless steels such as AISI 309 and 310, in which no martensite transformation occurred during tensile test. This hypothesis is as follows. Low SFE enhances planar dislocation motion, resulting in dislocation pileups at obstacles such as grain boundaries, inclusions, segregation and so on. If a metal or alloy contains hydrogen, hydrogen transport by dislocations occurs during the tensile tests. In the region of the pileup, the hydrogen concentrations would be high, leading to greater embrittlement [175].

In general, the effects of strain induced a'-martensite and SFE on HE are usually difficult to distinguish, since a'-martensite nucleation is closely-linked with the shear band formation associated with SFE, as described in section 5.2.2.1. Nevertheless, the relationship between the rate of shear band formation ( $\alpha$-parameter) and the probability of the formation of a'-martensite nuclei (B-parameter) are examined.

Fig. 5.4 shows 6 -parameter as a function of a-parameter obtained in this study. A dashed line in Fig. 5.4 shows the trend in the data. The 8-parameter is proportional to the $\alpha$-parameter in the range from 2 to 6.5. Above $\alpha=6.5$, it seems that the B-parameter becomes constant. In case of W11 and W19, the probability of the formation of a'-martensite nuclei is low, although the rate 


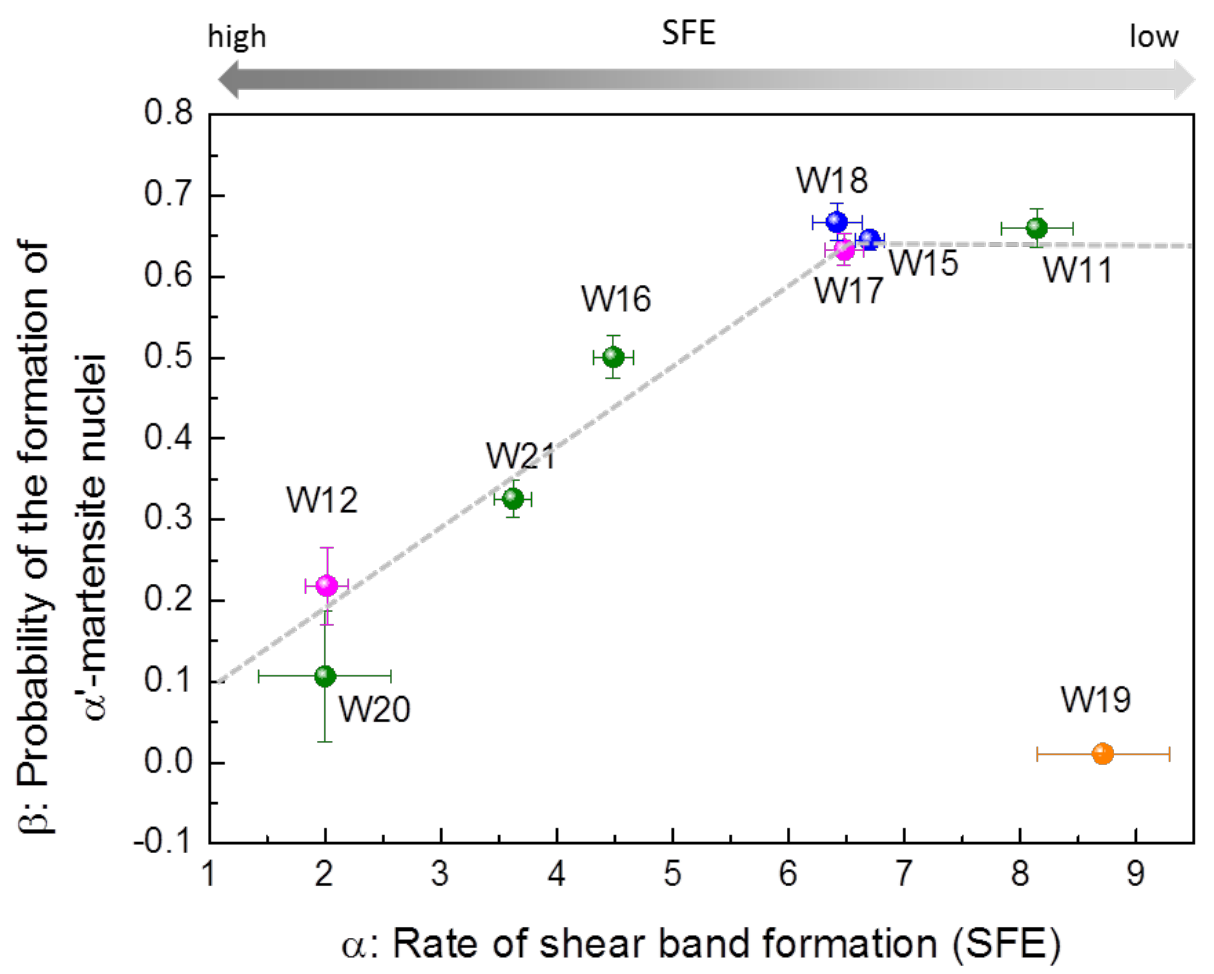

Fig. 5.4 Probability of the formation of $\alpha^{\prime}$-martensite nuclei as a function of rate of shear band formation (SFE). The colored points represent the type of oxide layer, (a) thick oxide: orange, (b) decaying sub-surface oxide: olive, (c) thin surface oxide: blue, and (d) high constant oxide level: magenta. Except for W19, $\beta$ parameter increase with increasing a parameter.

of shear band formation is high. This means that not all shear band intersections result in nucleation site for a'-martensite formation, since martensite transformation is limited by critical nucleation size [178].

As shown in Fig. 4.29, a close correlation between the B-parameter (probability of the formation of a'-martensite nuclei) and the RRA-value is found, while the less correlation is found between the a-parameter and the-RRA value. Consequently, martensite transformation plays a predominant role in the HE susceptibility of the low-nickel steels.

\subsubsection{Ni equivalent and $M_{\mathrm{d} 30}$ temperature}

The 6-parameter presented in the previous section was experimentally obtained as shown in Fig. 4.28. From the practical point of view, it is convenient to use indicators like the $\mathrm{Ni}$ content for the prediction of HE. But, it has already been mentioned that the Ni content itself does not seem to be 
available for this purpose (section 4.3.1 and section 5.2.2). Alternatively, we propose three empirical equations which are often used to describe the alloy's phase stability; (1) the Ni equivalent and (2) the $\mathrm{M}_{\mathrm{d} 30}$ temperature as given by Angel (Eq. (2-4) [35]) and (3) the $\mathrm{M}_{\mathrm{d} 30}$ temperature as given by Nohara (Eq. $(2-5)$ [36]) as described in section 2.1.2 and 2.1.3.2.

For the prediction of HE, several authors have proposed other empirical equations which were directly derived from the relationship between RRA-values (or RA-values) and alloy compositions [165,179]. In this study, however, we return to the concept of "phase stability" to interpret different experimental results reported so far. In the following, first, the accuracy of the above three equations for the phase stability is examined. After that, the most accurate equation will be further discussed to verify its validity for the prediction of HE in sections 5.2.2.3 and 5.2.2.4.

Fig. 5.5 shows the Ni equivalent plotted as a function of the B-parameter. No significant correlation between $\mathrm{Ni}$ equivalent and B-parameter can be seen in Fig. 5.5. This result is predictable since the Ni equivalent indicates the austenite stability as a function of the $\mathrm{Cr}$ equivalent in the Schaeffler diagram, suggesting the lack of the $\mathrm{Cr}$ contribution to the phase stability in this equation. 


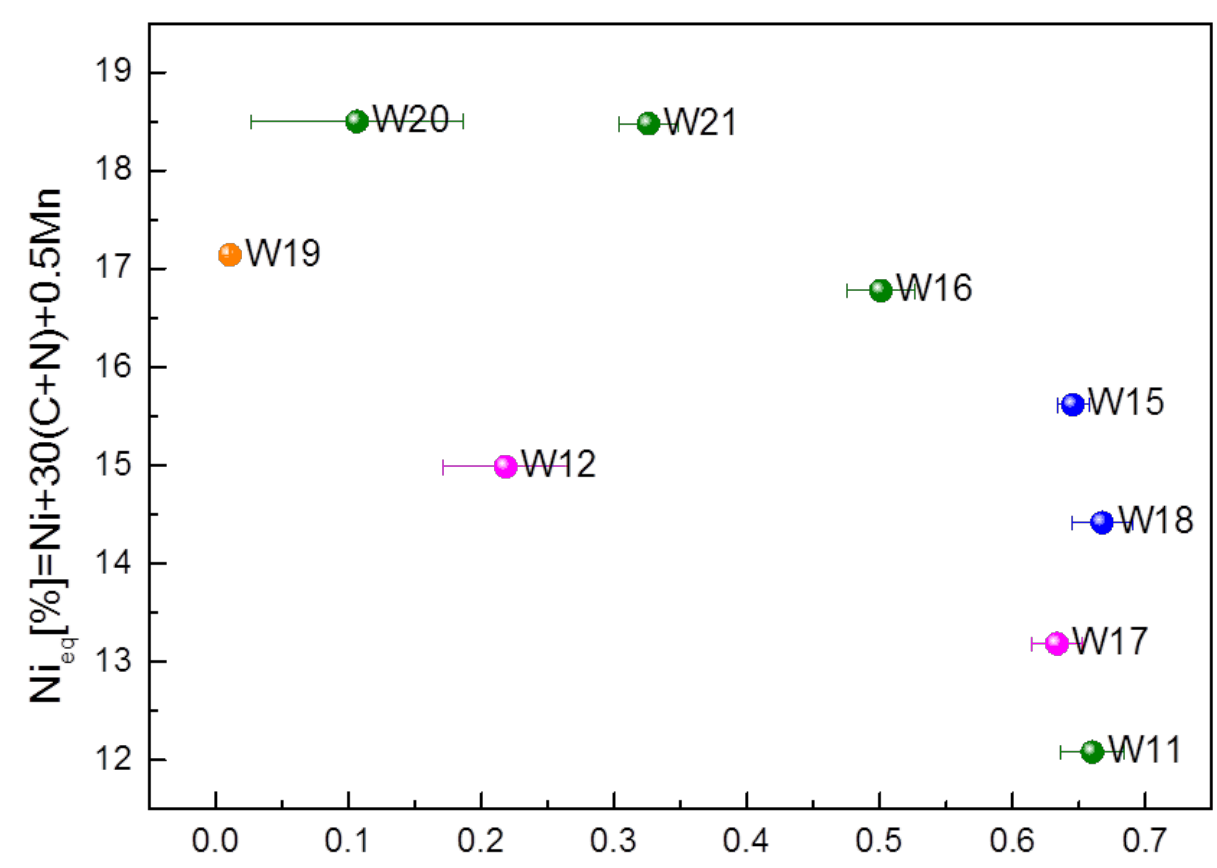

$\beta$ : Probability of the formation of $\alpha^{\prime}$-martensite nuclei

Fig. $5.5 \mathrm{Ni}$ equivalent plotted as a function of $\beta$ parameter. The colored points represent the type of oxide layer, (a) thick oxide: orange, (b) decaying sub-surface oxide: olive, (c) thin surface oxide: blue, and (d) high constant oxide level: magenta. No significant correlation between $\mathrm{Ni}$ equivalent and $\beta$ parameter.

Likewise, the $\mathrm{M}_{\mathrm{d} 30}$ temperature is calculated by the equation of Angel and Nohara and represented as a function of the 8-parameter in Fig. 5.6 and Fig. 5.8, respectively.

It is clear that the $\mathrm{M}_{\mathrm{d} 30}$ temperature by Nohara shows a close correlation with the experimentally obtained B-parameter, while $\mathrm{M}_{\mathrm{d} 30}$ temperature by Angel shows less correlation. The difference between two equations is the contribution of $\mathrm{Ni}$ and $\mathrm{Cu}$ to the austenite stability. In Angel's equation, the effect of Ni seems to be underestimated. Further, Nohara's equation takes into account the effect of $\mathrm{Cu}$, which has the same influence as nickel on the $\mathrm{M}_{\mathrm{d} 30}$ temperature. It also takes into account the influence of the grain size, which affects the martensite transformation. The grain size effect on the martensite transformation will be discussed in section 5.2.3.1. The departure of W19 from the trend line is attribute to the lack of the Al-contribution in the $M_{d 30}$ equation of Nohara. 


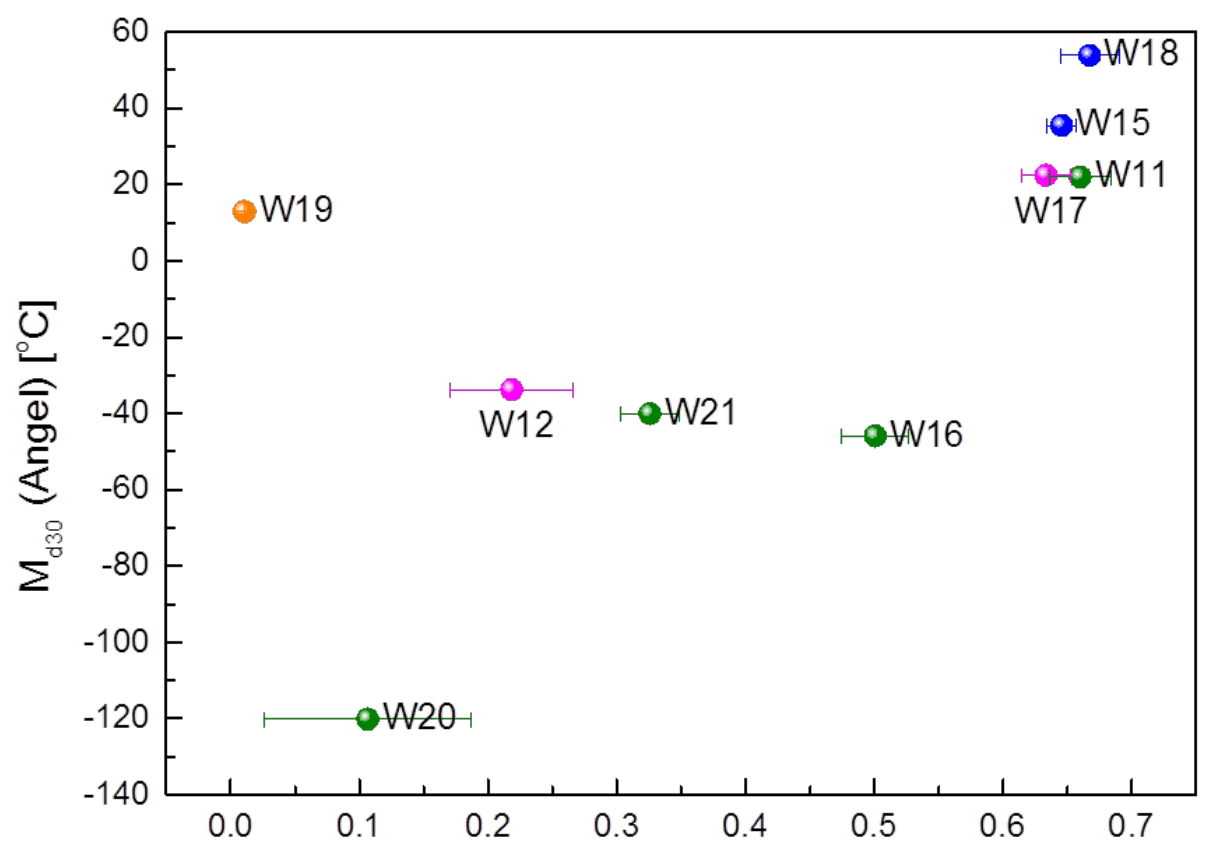

$\beta$ : Probability of the formation of $\alpha^{\prime}-$ martensite nuclei

Fig. 5.6 $M_{d 30}$ (Angel) plotted as a function of $\beta$ parameter. $M_{d 30}$ were calculated by Angel equation[35]. The colored points represent the type of oxide layer, (a) thick oxide: orange, (b) decaying sub-surface oxide: olive, (c) thin surface oxide: blue, and (d) high constant oxide level: magenta. A correlation between $M_{d 30}$ and $\beta$ is found

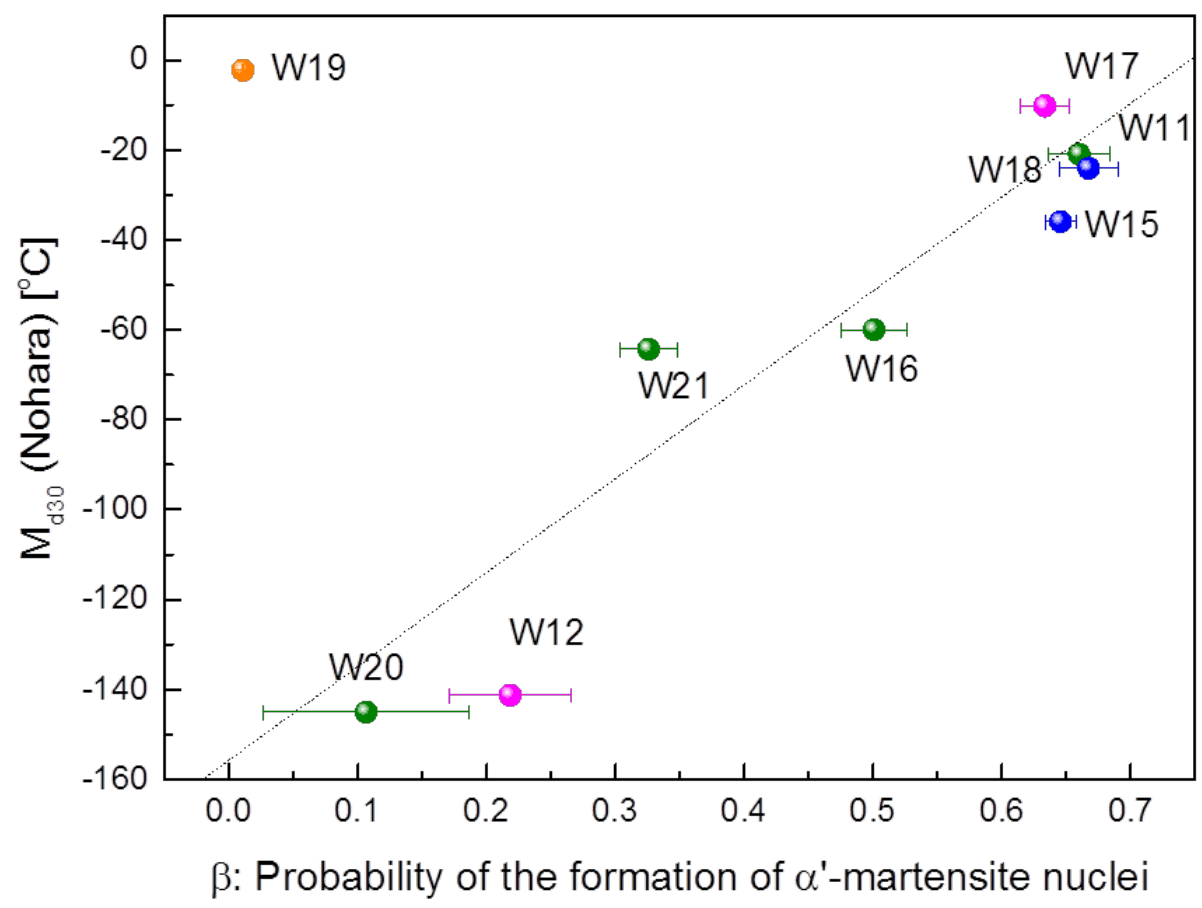

Fig. 5.7 $M_{d 30}$ (Nohara) plotted as a function of $\beta$ parameter. $M_{d 30}$ were calculated by Nohara equation [36]. The colored points represent the type of oxide layer, (a) thick oxide: orange, (b) decaying sub-surface oxide: olive, (c) thin surface oxide: blue, and (d) high constant oxide level: magenta. A close correlation between $M_{d 30}$ and $\beta$ is found 


\subsubsection{RRA-value vs $M_{d 30}$ at low temperature}

As deduced in the previous section, the $\mathrm{M}_{\mathrm{d} 30}$ temperature by Nohara shows a linear relationship with the B-parameter. This means that the $M_{\mathrm{d} 30}$ temperature by Nohara has a potential to serve as a good indicator for $\mathrm{HE}$ instead of $\mathrm{Ni}$ content. To evaluate the capability of the $\mathrm{M}_{\mathrm{d} 30}$ (Nohara) temperature as an indicator predicting the susceptibility to $\mathrm{HE}$, the RRA-values obtained at $-50{ }^{\circ} \mathrm{C}$ are plotted as a function of $\mathrm{M}_{\mathrm{d} 30}$ temperature to serve, as shown in Fig. 5.8. In this figure, comparable results reported by Fujii et al. [180] and by Michler [18] are also implemented. These results were obtained from similar tensile tests at low tempearture $\left(-40{ }^{\circ} \mathrm{C}\right.$ [180], $50{ }^{\circ} \mathrm{C}$ [18]) in high-presure hydrogen gas (45 MPa [180], $40 \mathrm{MPa}$ [18]) and in air to derive the RRA-values. In case of Fujii's data, there is no description of grain size. In order to plot their data, $50 \mu \mathrm{m}$ was adopted as grain size and dashed bars indicate the variation range for grain sizes varying from 5 to 200 $\mu \mathrm{m}$.

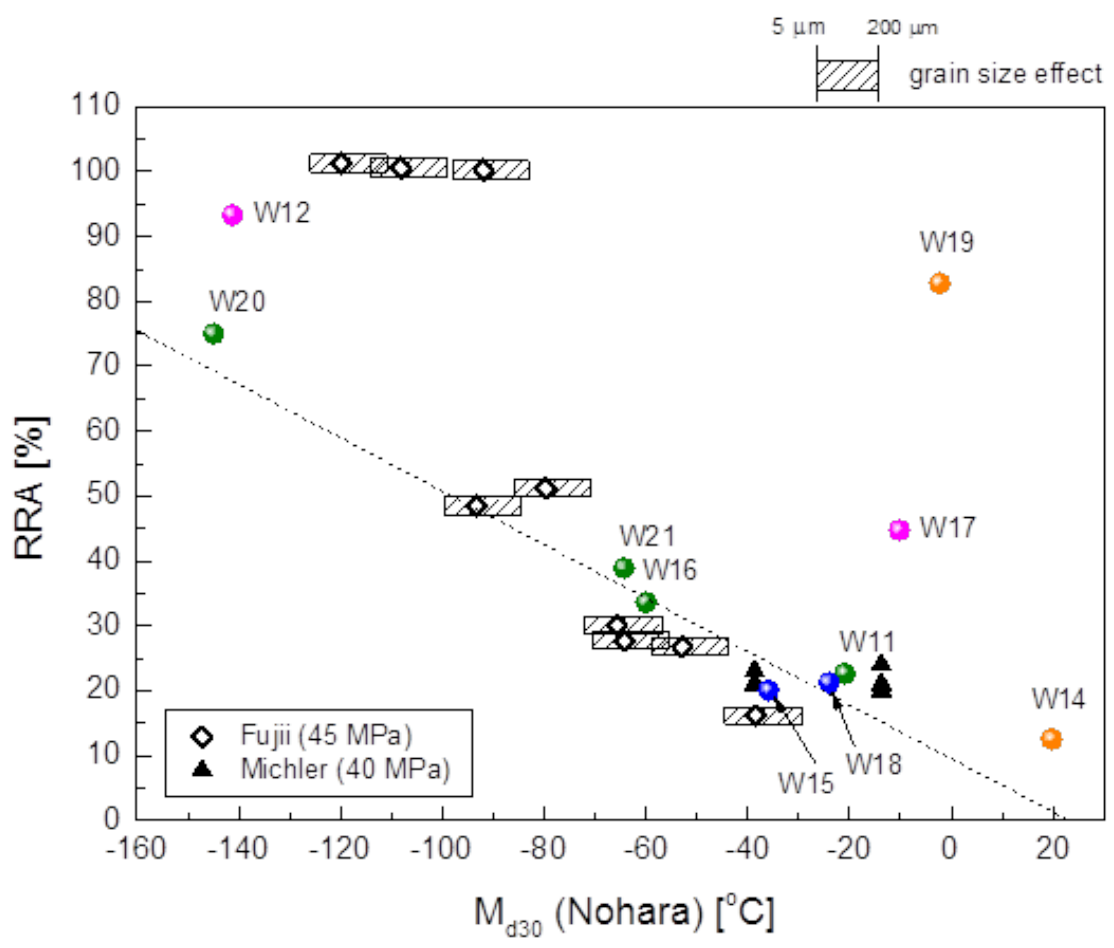

Fig. 5.8 RRA plotted as a function of $M_{d 30}$. The tensile tests were performed at $-50^{\circ} \mathrm{C}$ in $40 \mathrm{MPa}$ hydrogen and in air in this study. $\mathrm{M}_{\mathrm{d} 30}$ were calculated by Nohara equation [29]. Comparable results by Fujii et al. [149] and by Michler [18] are also represented. The colored points represent the type of oxide layer, (a) thick oxide: orange, (b) decaying sub-surface oxide: olive, (c) thin surface oxide: blue, and (d) high constant oxide level: magenta. A clear correlation was found above $-80^{\circ} \mathrm{C}$ of $\mathrm{M}_{\mathrm{d} 30}$ temperature. 
Dashed line shown in Fig. 4.29 provides an indication of trend. Except for $\mathrm{W} 12$, W14, W17 and W19, a clear correlation is found above $-80{ }^{\circ} \mathrm{C}$ of $\mathrm{M}_{\mathrm{d} 30}$ temperatures. It should be stressed that the departure of W14 and W19 from a linearity can be directly related to the lack of the Al-contribution in the $\mathrm{M}_{\mathrm{d} 30}$ equation of Nohara. In case of $\mathrm{W} 12$ and $\mathrm{W} 17$, these points also deviate somewhat from the linear relationship between the RRA-value and the B-parameter, as shown in Fig. 4.29. Below $-80{ }^{\circ} \mathrm{C}$, three points in Fujii also depart from the trend line. This feature will be discussed later.

\subsubsection{RRA-value vs $M_{\mathrm{d} 30}$ at room temperature}

Similarly, Fig. 5.9 shows RRA values for samples tested at room temperature versus $\mathrm{M}_{\mathrm{d} 30}$ for $\mathrm{W} 11$ and for similar data from Fukuyama [6,181], Michler [18], Walter [182] and Caskey [183]. The literature data were selected on the basis of the-RRA value vs $\mathrm{Ni}$ content, as reported by Michler (Fig. 4.15) [18].

Above $-80{ }^{\circ} \mathrm{C}$ in Fig. 5.9, a close correlation is found between the RRA-value and $\mathrm{M}_{\mathrm{d} 30}$. Exceptions are some data points surrounded by a circle. The general trend is indicated by a solid line. A large variation, indicated by a shaded region, is found at around $-80^{\circ} \mathrm{C}$. Below $-80^{\circ} \mathrm{C}$, the RRA-values are more or less constant and near $100 \%$. Only exception is steel group A.

The group surrounded by the circle is discussed now. W11 for $15 \mathrm{~min}$ contains the layer type of high constant oxide level as described in 4.2.2. The scatter of steel B is due to macro-segregation, as discussed by Michler et al. [18]. The scatter of group $\mathrm{C}$ is attributed to a large variation in grain size (10 - $350 \mu \mathrm{m}$ ) [183]. In this figure, however, the grain size effect on martensite transformation is taken into account. Further, steels of large grain size also show significantly higher RRA values compared to the trend line. Consequently, other potential factors such as surface roughness, macro-segregation, inclusions, etc. must be further considered. For instance, a tensile specimen with smooth surface improves by about $50 \%$ in the $R R A$-values compared to that with surface roughness of $R_{a}=0.4 \mu m$ [184]. 


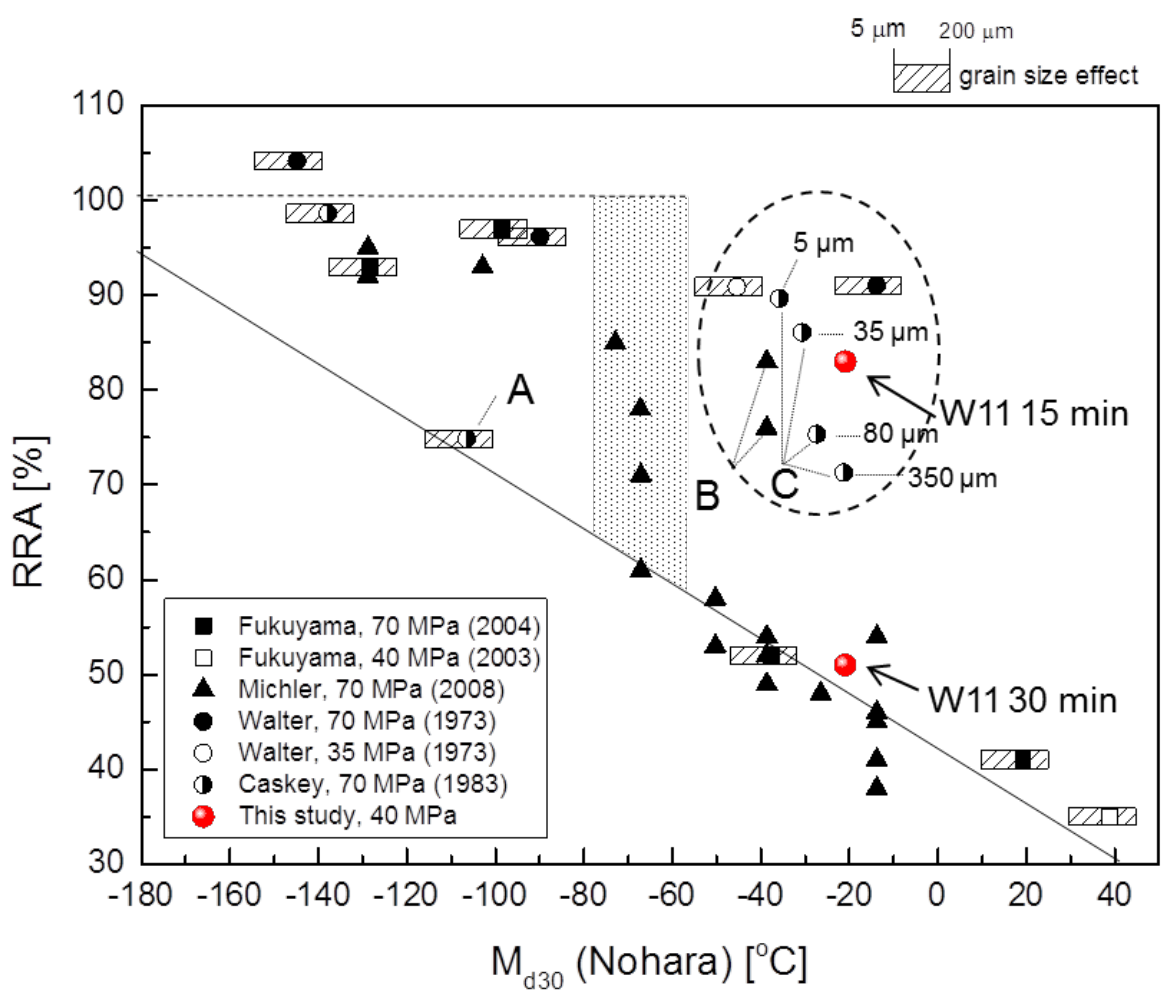

Fig. 5.9 RRA plotted as a function of $M_{d 30}$ including data from Fukuyama [6,181], Michler [18], Walter [182] and Caskey [183]. W11 were tensile tested at RT in 40 $\mathrm{MPa}$ hydrogen and in air. $\mathrm{M}_{\mathrm{d} 30}$ were calculated by Nohara equation [29].

Above $-80{ }^{\circ} \mathrm{C}$ of $\mathrm{M}_{\mathrm{d} 30}$, a close correlation is found except for some data points surrounded by a circle. A large variation is found at around $-80^{\circ} \mathrm{C}$ of $\mathrm{M}_{\mathrm{d} 30}$. Below $-80{ }^{\circ} \mathrm{C}, \mathrm{RRA}$ values are more or less constant near $100 \%$

Meanwhile, ASTM has published "Standard Test Method for Determination of Susceptibility of Metals to Embrittlement in Hydrogen Containing Environments at High Pressure, High Temperature, or Both" referred as G142-98 since 1998, in order to achieve comparable results among different researchers [185]. Currently, many researchers refer to this G142-98 standard for tensile tests in high pressure hydrogen atmosphere. It should be noted that the surface roughness of $R_{a}=0.38 \mu \mathrm{m}$ [20], mentioned in section 3.2.1.7, is within an allowance of G142-98, though a certain scatter is clearly present among the data. This means that the standard test method should be modified in line with new findings and, in fact, it was modified in 2004 and in $2011[100,186]$.

We, thus, have removed these old data in Fig. 5.9 and replotted again in 
Fig. 5.10., using recently reported RRA-values, obtained by tensile tests in high-pressure hydrogen gas / air, as a function of the calculated $\mathrm{M}_{\mathrm{d} 30}$ (Nohara) temperature. By this, following the standard G142-98 from 2011, should give us more reliable summary on the current discussion. For comparison, results on thermally pe-charged steels [165] are also plotted in this figure (blue data plots). In the following sections, we will discuss testing results in high-pressure hydrogen gas / air and by thermally pre-charging method separately.

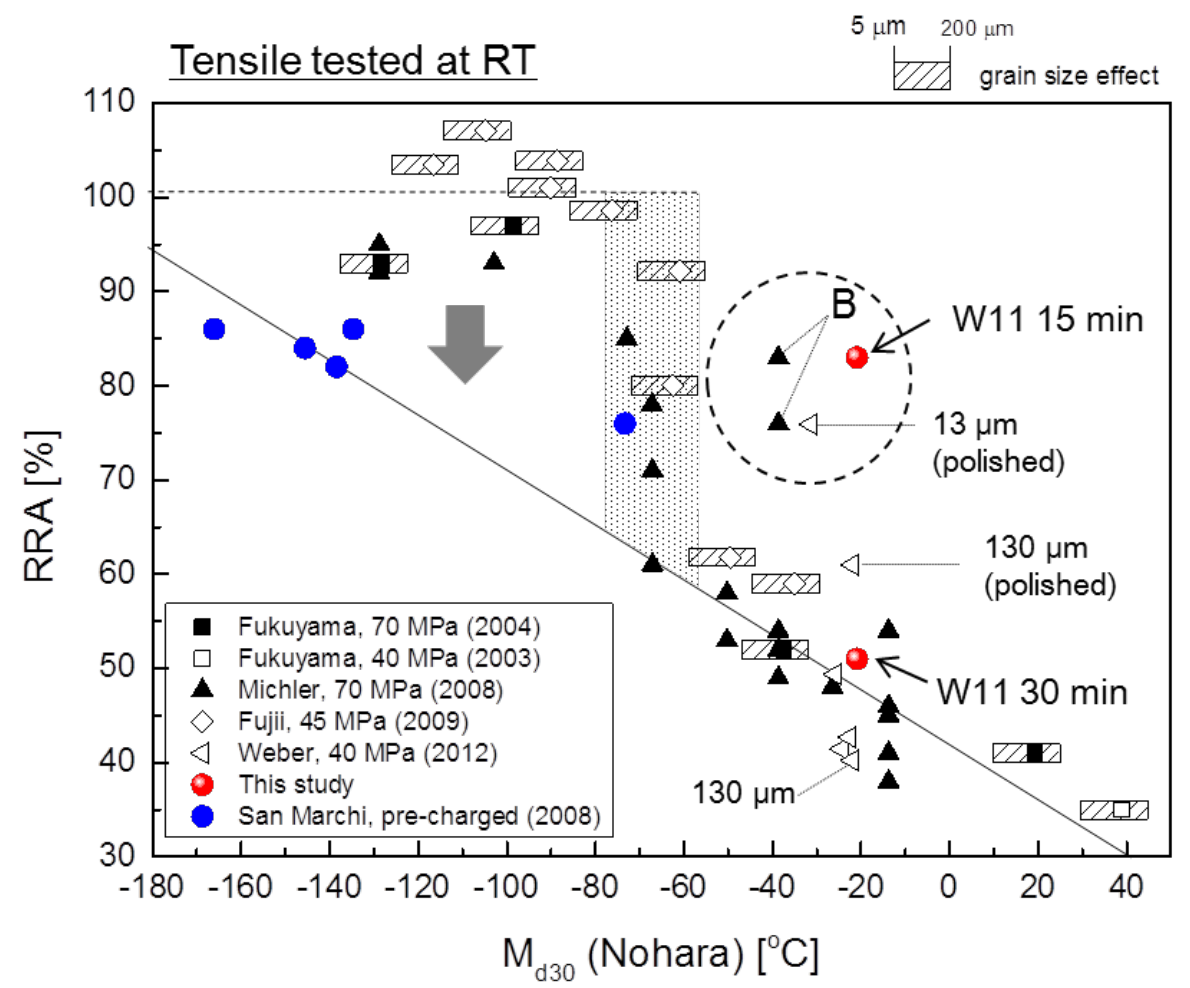

Fig. 5.10 RRA plotted as a function of $M_{d 30}$ including data from Fukuyama [6,181], Michler [18], Fuji [180], Weber [184] and San Marchi [165]. W11 were tensile tested at $\mathrm{RT}$ in $40 \mathrm{MPa}$ hydrogen and in air. $\mathrm{M}_{\mathrm{d} 30}$ were calculated by Nohara equation [29].

Above $-80{ }^{\circ} \mathrm{C}$ of $\mathrm{M}_{\mathrm{d} 30}$, a close correlation is found except for some data points surrounded by a circle. There is a threshold band from $-80^{\circ} \mathrm{C}$ to $-60{ }^{\circ} \mathrm{C}$ of $\mathrm{Md}_{30}$. There is a threshold band from $-80{ }^{\circ} \mathrm{C}$ to $-60^{\circ} \mathrm{C}$ of $\mathrm{Md}_{30}$. Below $-80{ }^{\circ} \mathrm{C}, \mathrm{RRA}$ values are more or less constant near $100 \%$. Below $-80{ }^{\circ} \mathrm{C}$, steels tested at $45 \mathrm{MPa}$ shows higher RRA compared with steels tested at $70 \mathrm{MPa}$.

RRA of steels with $M_{d 30}$ below the threshold band is quasi-static and could decrease with increasing hydrogen pressure for tensile tests. In steels with $M_{d 30}$ above the threshold band, martensite transformation plays a dominant role. 


\subsection{RRA-value in high-pressure hydrogen / air}

Fig. 5.10 exhibits quite similar features like Fig. 5.9. Here again, above $-80{ }^{\circ} \mathrm{C}$ of $\mathrm{M}_{\mathrm{d} 30}$, a close correlation is found, except for some data points surrounded by a circle. In the circle, the observed scatter of W11 annealed for $15 \mathrm{~min}$ is due to the layer type of high constant oxide level, as described in 4.2.2. The steel B is attributed to macro segregation [18]. In the steel with 13 $\mu \mathrm{m}$ grain size (polished), growth of cracks was stopped by obstacles such as grain boundaries [184]. This point will be discussed in detail in section 5.2.3.1. If we disregard these data points, a large variation is found at around $-80^{\circ} \mathrm{C}$, as shown as a clear RRA-value drop here. Below $-80{ }^{\circ} \mathrm{C}$, RRA values obtained in hydrogen atmosphere are more or less constant near $100 \%$.

Interestingly, steels tested at $45 \mathrm{MPa}$ [Fujii] show relatively high RRA values compared to that tested at $70 \mathrm{MPa}$ [Fukuyama, Michler]. This trend is significantly below $-80{ }^{\circ} \mathrm{C}$ of $\mathrm{M}_{\mathrm{d} 30}$. The steels with $\mathrm{M}_{\mathrm{d} 30}$ below $-80{ }^{\circ} \mathrm{C}$ are more stable against transformation from austenite to martensite. This means that martensite transformation hardly takes place in these steels. Thus, hydrogen transport may be retarded due to the lack of the rapid hydrogen diffusion path through the martensite phase. The hydrogen pressure during tensile tests at $45 \mathrm{MPa}$ is smaller than $70 \mathrm{MPa}$, leading to relatively high RRA-values compared with that at $70 \mathrm{MPa}$. In steels with $\mathrm{M}_{\mathrm{d} 30}$ below $-80^{\circ} \mathrm{C}$, the strain rates used in the cited study (with $3.3 \times 10^{-5}-$ $9.2 \times 10^{-5} \mathrm{~s}^{-1}$ in a similar order like the strain rate used here) are insufficient to achieve enough hydrogen concentration at lattice defects such as dislocations, interfaces etc. as the hydrogen diffusion time is short.

\subsection{RRA-values obtained by thermally pre-charging method}

San Marchi et al. have also addressed the shortage of hydrogen diffusion time and suggested a thermal pre-charging method to achieve a uniform and high concentration of hydrogen in relatively stable steels [165]. They reported on tensile test results for five different stainless steel specimens 
(modified AISI 316L) in annealed conditions [165]. These results are worthy to reveal the effect of internal hydrogen on the RRA-values, as shown in Fig. 5.10. (In San Marchi's study, they evaluated the effect of hydrogen by using reduction in area (RA). Fig. 5.10 represents the calculated RRA-values which were computed by dividing the RA values of steel with pre-charging by the RA-value of steel without pre-charging).

As expected, thermally pre-charged steels exhibit lower RRA values than that tensile tested in a high pressure hydrogen atmosphere. It seems that there is a threshold band from $-80{ }^{\circ} \mathrm{C}$ to $-60{ }^{\circ} \mathrm{C}$ of $\mathrm{M}_{\mathrm{d} 30}$. Consequently, the steels with $\mathrm{M}_{\mathrm{d} 30}$ below the threshold band seem to be relatively stable, because the strain rate is insufficient for hydrogen diffusion. This means that RRA-values obtained by tensile tests in a high pressure hydrogen atmosphere are "quasi-static" and may decrease by increasing the applied hydrogen pressure, as indicated by an arrow in Fig. 5.10. In steels with $\mathrm{M}_{\mathrm{d} 30}$ above the threshold band, martensite transformation plays a dominant role, as described in section 5.2.1.

\subsubsection{Influence of thermal history}

In the previous sections, we concluded that the martensite phase transformed during tensile tests plays a major role in $\mathrm{HE}$ of the austenitic steels. This effect of martensite formation was taken into account in Fig. 4.15, Fig. 4.29, Fig. 5.8 and Fig. 5.10, to thoroughly evaluate different RRA data. However, some data points are still far away from the general trend line. For instance, in Fig. 5.10, the sample W11 annealed for 15 min was found to be rather insusceptible against $\mathrm{HE}$, while W11 annealed for 30 min follows the trend line. This suggests that thermal history also becomes an important factor to HE. The difference in annealing time changes the grain size, the dislocation density, the type of the oxide layer and so on. Thus, in this section, influences of these micro-structural properties on the HE will be discussed based on the results of Fig. 4.15, Fig. 4.29 and Fig. 5.10. 


\subsubsection{Gain size}

Firstly, we focus on the influence of the grain size associated with the martensite transformation on HE. As expressed in the $\mathrm{M}_{\mathrm{d} 30}$ (Nohara) equation (Eq. (2-5) [36]), $\mathrm{M}_{\mathrm{d} 30}$ temperature decreases with decreasing grain size, in other words, martensite transformation is impinged by grain refining. The question arises if grain refining improves the resistance of steels to HE or not. A few researchers have systematically studied the effects of grain size on the HE [160,163-165].

Moody et al. studied the effect of hydrogen on the fracture toughness of the $\mathrm{Fe}^{-\mathrm{Ni}}$-Co superalloy IN903 (austenite) with grain sizes ranging from 28 to $200 \mu \mathrm{m}$ [187]. They found that fracture toughness was independent of the grain size.

In contrast, Eliezer et al. reported that the resistance to HE of austenitic stainless steels (AISI 304L, 316L, 316, 321 and 347) improves by decreasing the grain size $[188,189]$. Recently, Weber et al. investigated the effect of grain size on the formation of strain-induced a'-martensite and HE of AISI 304L at room temperature. Their results are also represented in Fig. 5.10 and, as can be seen, $13 \mu \mathrm{m}$ (polished) shows higher RRA values than $130 \mu \mathrm{m}$ (polished) [184], supporting the results of Eliezer et al.

The improvement of resistance to $\mathrm{HE}$ by grain size refining seems to associate with the formation of strain-induced a'-martensite during tensile tests. However, the martensite transformation curves of 13 and $130 \mu \mathrm{m}$ (polished), obtained under tensile testing at room temperature and in air, indicate that the amount of martensite is more or less independent from grain sizes [184]. This may seem curious from the perspective of the grain size effect in Nohara's equation. But, it should be considered that the tensile tests in Ref. [184] were performed at room temperature. In case of AISI 304 $\left(\mathrm{M}_{\mathrm{d} 30}:-20^{\circ} \mathrm{C}\right)$, significant grain size effect on martensite transformation was observed in the tensile test temperature range from -20 to $-50{ }^{\circ} \mathrm{C}$, while a small effect is found above $-10^{\circ} \mathrm{C}$ [36]. Consequently, the grain size effect on martensite transformation is negligibly small at room temperature when the $\mathrm{M}_{\mathrm{d} 30}$ temperature of steels is below that. Thus, only if tensile tests are 
carried out at low temperature, the grain size effect on martensite transformation must be taken into account.

The improvement of the resistance to $\mathrm{HE}$ by grain refinement arises from other causes rather than the martensite transformation. In the study by Weber et al., the improvement of resistance to $\mathrm{HE}$ by grain size refining is attributed to obstacles such as grain boundaries and segregation bands where growth of hydrogen-assisted cracks is stopped. [184].

Now, we come back to the results on W11 annealed for 15 min and $30 \mathrm{~min}$, respectively, as shown in Fig. 4.15. Again, the results were obtained by tensile tests performed at room temperature. The improvement of the RRA-value was found on the sample annealed for 15 min when compared to that annealed for $30 \mathrm{~min}$. The effect of heat treatment time on the grain size is significantly small, since the grain size in $15 \mathrm{~min}$ and $30 \mathrm{~min}$ is almost identical. (The mean grain size was $47 \mu \mathrm{m}$ for $15 \mathrm{~min}$ sample and $53 \mu \mathrm{m}$ for 30 min sample, respectively [20].) Therefore, the difference in the data of W11 annealed for $15 \mathrm{~min}$ and $30 \mathrm{~min}$ samples must arise from other contributions than the grain size effect.

\subsubsection{Dislocation density and dislocation substructure}

The difference in the annealing time of $15 \mathrm{~min}$ and $30 \mathrm{~min}$ also changes the number of dislocations formed during sample machining prior to the annealing process. XRD measurements shown in section 4.2.2.3 verified that the dislocation density in W11 annealed for $30 \mathrm{~min}$ is one order of magnitude lower than that in W11 annealed for 15 min (Table 4.3). This is in good agreement with the expected general recovery effect [190].

Now we consider the dislocation-hydrogen interaction during tensile tests. Regarding the HELP theory (2.2.1.4), hydrogen may facilitate the dislocation motion, leading to HE of steels. From this point of view, it can be expected that W11 annealed for 30 min would be more susceptible to HE than W11 annealed for $15 \mathrm{~min}$, because the dislocation density of $30 \mathrm{~min}$ sample is higher than that of $15 \mathrm{~min}$. But, the RRA-value of the sample 
annealed for $30 \mathrm{~min}$ is lower than that annealed for $15 \mathrm{~min}$ (Fig. 4.15). This is in contrast to the mentioned assumption based on the HELP theory.

The higher RRA-vaue for the sample annealed for 15 min could be interpreted in terms of the dislocation substructures. Louthan et al. investigated the effects of high energy rate forging (HERF) process on HE [175]. By using this process, dislocation tangles and cell structures form in austenitic steels without the formation of martensite phase. The specimens were pre-charged in $69 \mathrm{MPa}$ hydrogen gas and tensile tested in air. They reported on $68 \%$ reduction in area (RA) observed in HERFed AISI 304L as compared with $36 \%$ RA in annealed AISI 304L [175]. This means that HERF process (prior to hydrogen charging) improves the resistance to HE of AISI 304L. The effects of HERF on HE could be ascribed to the initiation of dislocation tangles and cellular structures that impede hydrogen transport by dislocation motion [175].

In this part of the study, we analyzed the dislocation substructures in the samples of the as received, the solution annealed for $15 \mathrm{~min}$, and solution annealed for $30 \mathrm{~min}$ by TEM (Fig. 4.18). In the samples that was solution annealed for $15 \mathrm{~min}$, the dislocation tangles are partially observed, while only some planar dislocations are found in the solution annealed for $30 \mathrm{~min}$. It seems that the higher RRA value for 15 min sample can be explained by the assumption that tangled dislocations and celluar structures inhibit dislocation motion [175]. However, this explanation cannot be immediately applied to this study, since the dislocation substructure observed in W11 annealed for 15 min sample differs from Louthan's study. In the TEM image of our sample (Fig. 4.18 (d)), the cellular dislocation structure is not evidently seen. The tangle dislocations were only partially observed. In contrast, HERFed AISI 304L shows strong cellular dislocation structure [175].

A later study by West suggests that the HERF process improves the resistance to $\mathrm{HE}$ only in precharged conditions [191]. Under tensile tests at high hydrogen pressure in the range from 69 to $173 \mathrm{MPa}$, HERFed steels were severely embrittled compared with annealed steels, and the degree of 
embrittlement increased with increasing hydrogen test pressure [191]. This means, at high hydrogen pressure, tangle dislocations and cellular dislocation structure do not have any beneficial effect in minimizing the susceptibility of steels to HE. In this study, tests were carried out at $40 \mathrm{MPa}$ hydrogen pressure. This pressure is lower than that used by West et. al. However, the extrapolation of $69 \mathrm{MPa}$ to $40 \mathrm{MPa}$ predicts that dislocation structures do not play a beneficial role in resistance to $\mathrm{HE}$.

The impact of the HERF process associated with dislocation substructures is still open question, since HERF processes might change microstructures including grain sizes as well. Further investigations on the effect of dislocation structures on HE are necessary.

\subsubsection{Oxide layer}

\subsection{Summary of four types of oxide layer}

Before discussing the influence of the oxide layer on $\mathrm{HE}$, the four different types of oxide layers determined in section 4.3.2 are summarized in Fig. 5.11. In this figure, the three-dimensional distribution of elements is also schematically illustrated based on the secondary ion images. (The secondary ion images of each specimen can be found in Fig. 4.23 for W14 and W19, and in Appendix 8.5 for W11, W12, W16, W17, W20 and W21, and Fig. 4.17 for W11, when annealed for $15 \mathrm{~min}$.) Again, all specimens were annealed for $30 \mathrm{~min}$, except for the W11 specimen annealed for $15 \mathrm{~min}$. 


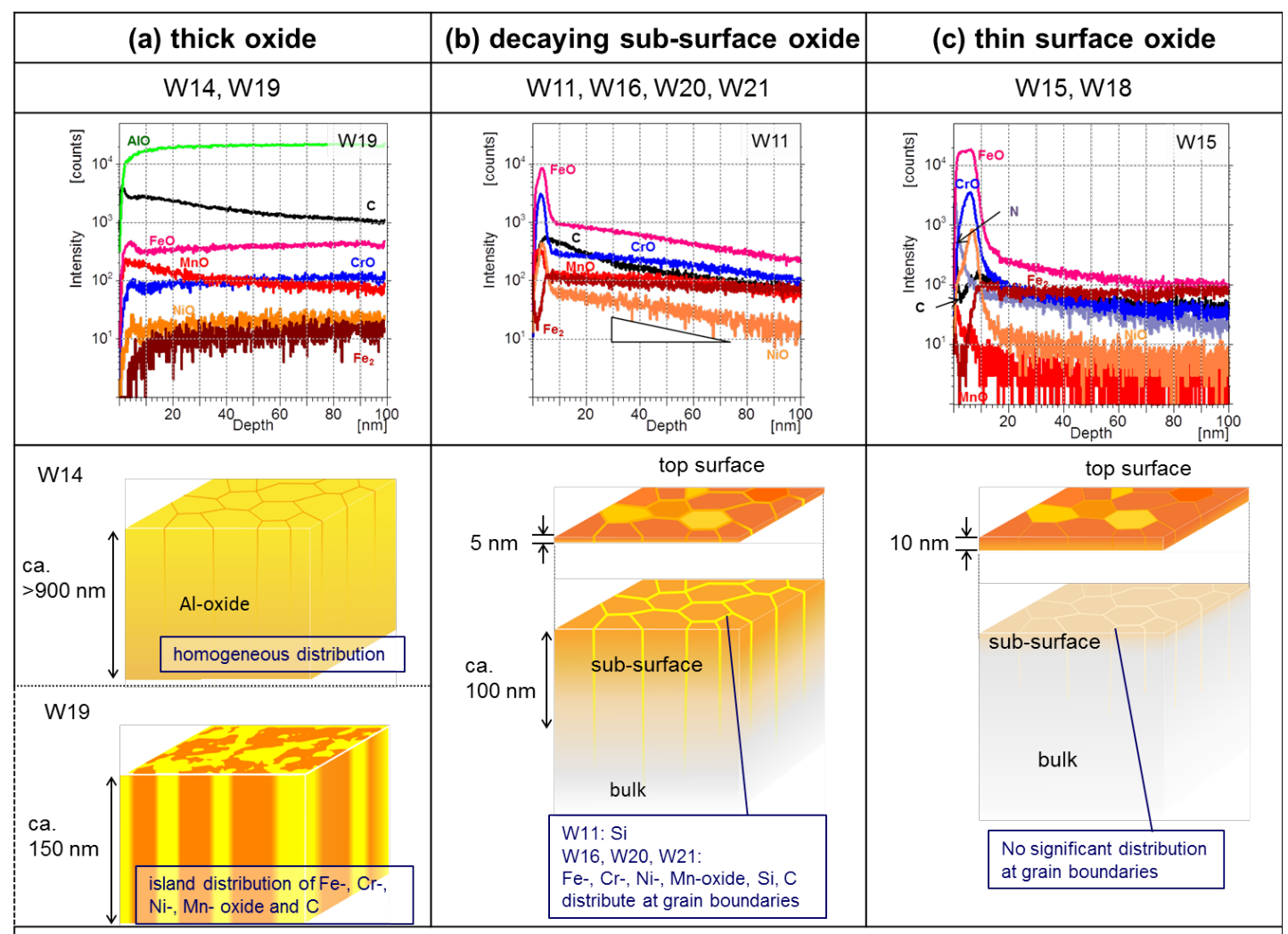

(d) high constant oxide level

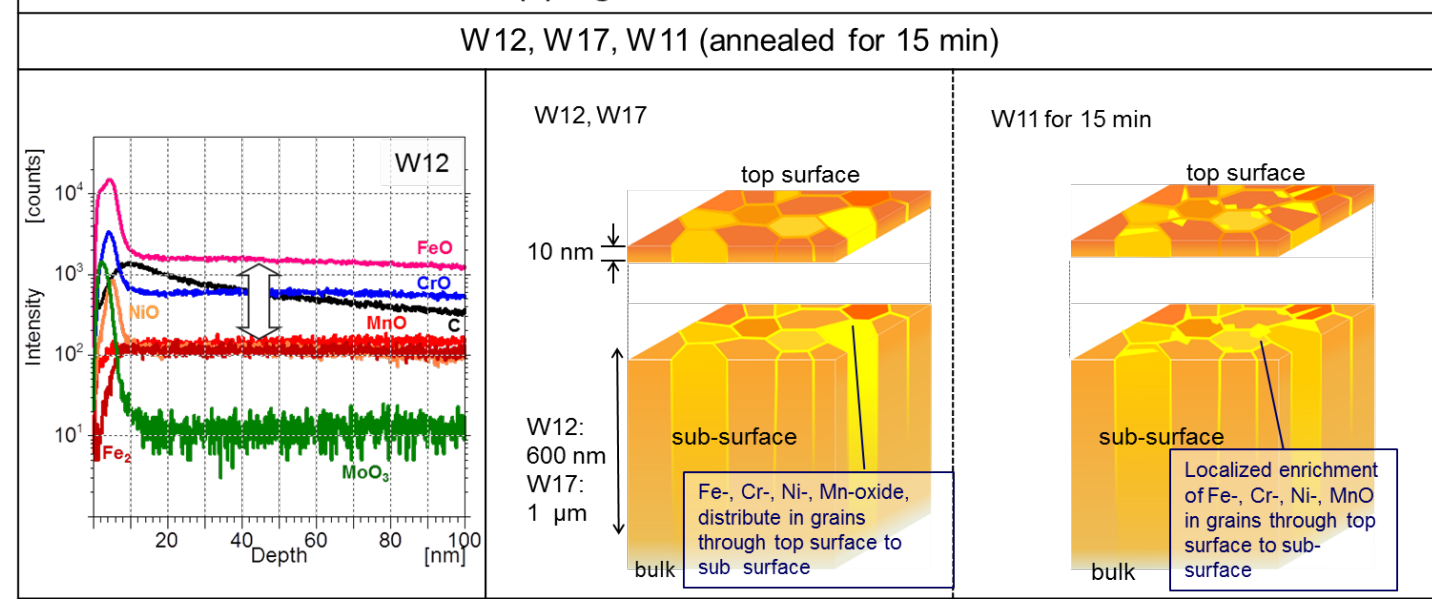

Fig. 5.11 Summary of four types of oxide layer formed on W11-W21 steels

The distribution features of each type of oxide are briefly summarized as follows.

(a) Thick oxide

- W14: Relatively homogeneous distribution of each element.

- W19: Island distribution of $\mathrm{Fe}^{-}, \mathrm{Cr}^{-}, \mathrm{Ni}^{-}, \mathrm{Mn}^{-}$oxide and $\mathrm{C}$.

(b) decaying sub-surface oxide

- W11: Si distributes at grain boundaries in sub-surface region. 
- W16, W20, W21: Fe-, Cr-, Ni-, Mn־oxide, Si, and C significantly enrich in grain boundaries in sub-surface region.

(c) thin surface oxide

- W15, W18: Relatively homogeneous distribution of each element is found in both surface and sub-surface region.

(d) high constant oxide level

- W12, W17: $\mathrm{Fe}^{-}, \mathrm{Cr}^{-}, \mathrm{Ni}^{-}, \mathrm{Mn}$-oxide localize in some grains from top surface to sub-surface region.

- W11 for 15 min: Partial localization of $\mathrm{Fe}^{-}, \mathrm{Cr}^{-}, \mathrm{Ni}^{-}, \mathrm{Mn}-$ oxide in some grains is found from top surface to sub-surface region.

\subsection{Influence of oxide layer on HE}

To elucidate the influence of the oxide layer on HE, we focus on the results of the $R R A$-values vs $\mathrm{Md}_{30}$ (Nohara) which was found to be a strong indicator for the impact of martensite transformation on HE (section 5.2.2). Fig. 5.8 shows the RRA values for the low-nickel steels as a function of $\mathrm{Md}_{30}$. Tensile tests were performed at $-50{ }^{\circ} \mathrm{C}$ in $40 \mathrm{MPa}$ hydrogen. In this figure, a threshold band is found at $-80^{\circ} \mathrm{C}$ of $\mathrm{M}_{\mathrm{d} 30}$. Above $-80^{\circ} \mathrm{C}$ of $\mathrm{M}_{\mathrm{d} 30}$, W17 and $\mathrm{W} 19$ show higher RRA-values with respect to the trend line.

As discussed in the previous sections, any departure from the trend line indicates that the $\alpha$-martensite formation alone cannot explain $\mathrm{HE}$ of austenitc stainless steels (see section 5.2.2.3 and 5.2.2.4). The grain size impact on $\mathrm{HE}$ is considered to be small in this study, since all samples have similar mean grain sizes in the range between 40 and $60 \mu \mathrm{m}$. Dislocation density in samples is in the range of $10^{12}$ to $10^{14}$ (Fig. 4.27), and does not systematically scale with the RRA-values (see Fig. 4.27 and section 5.2.3.2). This suggests that dislocations existing prior to the tensile tests are not solely responsible for the scatter observed in Fig. 5.8.

Concerning the influence of oxide layers on HE, we found by looking at Fig. 5.8 that the oxide type; (b) decaying sub-surface oxide and (c) thin surface oxide follow the trend line, while RRA value for W19 ((a) thick oxide) and W17 ((d) high constant oxide level) are far above the trend line. 
Interestingly, this feature was also found in the tensile test results at room temperature shown in Fig. 5.9. As mentioned before, the RRA-value of the annealed for 15 min sample shows a higher RRA value compared to that when annealed for $30 \mathrm{~min}$ (Fig. 5.9). SIMS results show that the oxide layer on W11 annealed for 15 min sample is of the "high constant oxide level" type, while decaying sub-surface oxide was found on $30 \mathrm{~min}$ sample. Thus, HE of samples having a "high constant oxide level" is influenced by the surface oxide.

According to the scheme of Fig. 1.2 [4], for the HE of alloys, the following processes occur; (1) Hydrogen transport, (2) Hydrogen adsorption on the surface, (3) Dissociation from molecular hydrogen to monoatomic hydrogen, (4) Hydrogen entry, (5) Hydrogen diffusion to the fracture propagation zone in the stress field, and (6) Hydrogen trapping at defects such as dislocations, vacancies, grain boundaries, etc.

Often, oxide layers work as protective layers to hydrogen access because oxide layers may prevent the dissociation of hydrogen molecules or prevent hydrogen diffusion. However, during tensile tests, the oxide layers break due to the sample elongation. Consequently, dissociation of hydrogen molecules occurs on a fresh surface created by cracking the oxide layers. The crack-like distribution consisting of the oxide and the fresh surface is found in Fig. 3.13. This image was taken near the screw parts of test piece which is least elongated after tensile testing. Thus, the distribution of the oxide and the fresh surface in Fig. 3.13 might be regarded as a surface formed in an early stage of tensile testing. Meanwhile, this crack-like distribution suggests also that the remaining oxide limits the entry site for hydrogen.

Regarding the mechanical properties of oxides, the oxide itself is inherently brittle. The thermal expansion of oxides is smaller than that of metals, resulting in local elastic compression in the oxides and tension in the metals during the cooling process [192]. This could lead either the brittle fracture or spalling of the oxide layer during tensile tests. However, the oxide thickness and the external strain strongly influences the failure mode of the oxide layer, since brittleness is regarded as a size-dependent concept [193]. 
The failure map, which is represented failure strain as a function of oxide thickness, indicates that a thin oxide layer of less than several hundred nm fails in a ductile manner [193]. Thus, the deformation mode of the studied oxide layers follows the same manner as the bulk material in the early stages of tensile testing.

The ratio between the specimen elongation and the oxide layer thickness must also be considered. In this study, the strain rate of $5.5 \times 10^{-5} \mathrm{~s}^{-1}$ was applied for the gauge length of $30 \mathrm{~mm}$. Thus, the cross-head displacement corresponding to specimen elongation is $1.65 \mu \mathrm{m} \cdot \mathrm{s}^{-1}$. In this regard, the oxide layer thickness among the samples indicates that (b) and (c) type of the oxide layer, which possess the oxide layer region in the range from 5 to $100 \mathrm{~nm}$, break within several seconds and hydrogen could enter through the fresh surface. Afterwards, crack growth occurs. The onset of such cracking can be closely linked to the mechanical properties of the oxide on and in the matrix. Meanwhile, in (d) type of the oxide layer, the high constant oxide signal continues in the range from $600 \mathrm{~nm}$ to $1 \mu \mathrm{m}$ in depth. This type of oxide may suppress the hydrogen entry in the course of cracking at the early stage of tensile testing.

It should be considered that the (a) type of the thick oxide layer is not a similar case, although the RRA-value for W19 is higher than the trend line. The tensile test was explicitly carried out after removing the thick oxide layer. The RRA-value for W19 without thick oxide is almost same as that for W19 with the thick oxide layer [20]. As a result, we conclude that the thick aluminum oxide layer on W19 is not causing the additional increase of the RRA-value. Further, in case of W12, the high RRA-value is not only the relatively thick oxide layer, but also attributed to the test conditions. As described in section 5.2.2.4.2, stable steels with $\mathrm{M}_{\mathrm{d} 30}$ below the threshold band show "quasi-static" RRA-values, when the strain rate is insufficient for hydrogen diffusion.

In the light of the above discussion, it should be emphasized that the dominant contribution of the oxide layer on $\mathrm{HE}$ is found in the results of W17 and in W11 when annealed for a $15 \mathrm{~min}$. Lateral distribution of oxides also 
influences the increase in their RRA value. The localized enrichment of oxides in grains is found as shown in Fig. 4.17 and Appendix 8.5. This enrichment becomes more pronounced with depth. From a macroscopic point of view, the lateral enrichment of oxides might work as obstacles that block crack propagation [18,184]. From the microscopic point of view, however, dislocation motion impede at obstacles and dislocation pile-ups occur, resulting in the stress concentrations at these sites [191,194]. Although the results of this study suggest that the lateral enrichment of oxides influence on $\mathrm{HE}$, more research is required to elucidate the crack propagation behavior at obstacles including oxides in both macroscopic and microscopic scale.

The other conceivable source for the improvement of the RRA-value is the influence of residual oxygen in environment of the tensile test system. As described in section 2.2.3.2, the gas purity affects the mechanical performance. The presence of impurity gas may inhibit the entry of hydrogen into materials. Especially, oxygen gas is preferentially absorbed compared with hydrogen and a quick re-passivation of fresh surfaces can occur [47]. In this study, only pure hydrogen gas $\left(\geq 99.9999 \% \mathrm{H}_{2}\right)$ was used, with an oxygen content of $0.2 \mathrm{ppm}$ and other impurities of less than $0.5 \mathrm{ppm}$ [73]. Thus the influence of gas impurities during the tests in this work can be neglected. 


\section{Summary}

As current high-cost $\mathrm{Ni}$ containing stainless steels are considered to be replaced by low-nickel content counterparts in the future, a detailed and fundamental investigation of $\mathrm{HE}$ behavior from various aspects is of high importance. In this thesis, therefore, low-nickel austenitic stainless steels were studied and their HE behavior was investigated in details. Careful consideration was made especially to determine the impact of the oxide layers on $\mathrm{HE}$, because, in general, oxide layers on steels work as a substantial barrier against hydrogen entry. For the analysis of oxide layers on the studied alloys, Time-of Flight Secondary Ion Mass Spectrometry (TOF-SIMS) technique was exclusively employed. This technique provides three-dimensional information for all elements with high sensitivity in the ppb-range and high depth resolution, enough to resolve nm-layers from the top surface to bulk regions. The results were supported by studies using X-ray diffraction (XRD), Energy Dispersive X-ray spectrometry (EDX) and Transmission Electron Microscope (TEM) to clarify significant impacts. Furthermore, data were provided from third parties regarding the embrittlement accessibility describing relative reduction of area (RRA) and martensite transformation curves.

All specimens were solution annealed (at $1050{ }^{\circ} \mathrm{C}$ or $1150{ }^{\circ} \mathrm{C}$ ) to restore the material, since the presence of the surface a'-martensite formed during the specimen manufacturing increases the susceptibility to $\mathrm{HE}$ of alloys (section 4.2.1). Tensile tests were performed in air and in $40 \mathrm{MPa}$ hydrogen gas either at room temperature or at $-50{ }^{\circ} \mathrm{C}$ to achieve maximum embrittlement effects. These tensile test conditions were adopted for the environment of practical hydrogen gas tank systems which are proposed by automakers (chapter 1, Fig. 2.9 and Fig. 2.11). The resistance of steels to HE, expressed as relative reduction of area (RRA), is reported to be proportional to the Ni content. However, the scatter of RRA data vs Ni content was found to be large, also for the sample in this study, especially below $11 \mathrm{wt} \% \mathrm{Ni}$ (Fig. 4.20). The RRA value is even found to change for sample of identical $\mathrm{Ni}$ 
content (W11 annealed for $15 \mathrm{~min}$ and for $30 \mathrm{~min}$, see Fig. 4.15). Thus, this relation was judged to be insufficient at least for the steels handled in this study. This calls for a new indicator instead of the Ni content.

Possible sources regarding the wide scatter of the RRA-values vs $\mathrm{Ni}$ content are the complexity of the variables, i.e., phase stability (a'-martensite content), the grain size, the dislocation density and the dislocation structure prior to the tensile test, and the oxide layer. To evaluate the contribution of the oxide layers on $\mathrm{HE}$, these variables are considered first and, summarized as below.

\section{Phase stability ( $\alpha$ '-martensite content)}

First of all, the martensite transformation of the austenite phase was found to play a dominant role in $\mathrm{HE}$ of the low-nickel austenitic stainless steels. This can be directly related to the fast hydrogen diffusion in the a'-martensite phase (bcc) and the higher solubility in the austenite matrix (fcc) (Fig. 2.14 and Fig. 2.15). To determine the impact of a'-martensite on the RRA-values, a new indicator is here suggested to be necessary.

\section{- RRA vs $\alpha^{-}$and B-parameter by Olson Cohen equation}

Martensite transformation curves for the studied alloys were fitted by the Olson-Cohen equation. Two parameters were obtained: The rate of shear-band formation ( $\alpha$-parameter) and the probability of the formation of a'-martensite nuclei (B-parameter). The RRA-values vs B-parameter represents a close linear relationship (Fig. 4.28), while less linearity was found in the RRA-values vs a-parameter (Fig. 4.29). This can be considered that the probability of the formation of $\alpha$ '-martensite nuclei (B-parameter) governs the susceptibility of these alloys to hydrogen atmosphere.

\section{- Evaluation of the new indicator $\mathrm{M}_{\mathrm{d} 30}$ (Nohara)}

Based on the above results, and, for comparison of the results obtained in this study with literature data, the practical effectiveness of three empirical equations was tested: (1) Ni equivalent, (2) $\mathrm{M}_{\mathrm{d} 30}$ temperature by Angel (3) $\mathrm{M}_{\mathrm{d} 30}$ temperature by Nohara. All equations were tested with respect to the 
B-parameter in the Olson-Cohen equation. As a result, the $\mathrm{M}_{\mathrm{d} 30}$ temperature by Nohara was found to highly correlate with the B-parameter and, therefore, was adapted as the new alternative indicator for the prediction of HE (Fig. 5.5 - Fig. 5.7).

\section{- Data in the light of the new indicator: the threshold band}

The RRA-values obtained in this study and reported data were carefully compared by using the new indicator $\mathrm{M}_{\mathrm{d} 30}$ (Nohara) temperature, as summarized in Fig. 5.8 - Fig. 5.10. Three distinct regions were evidently found in the RRA-values versus $\mathrm{M}_{\mathrm{d} 30}$; (1) above $-80^{\circ} \mathrm{C}$ of $\mathrm{M}_{\mathrm{d} 30}$, RRA-values decrease with increasing $\mathrm{M}_{\mathrm{d} 30}$ temperature, (2) around $-80^{\circ} \mathrm{C}$, a large variation in RRA-values (which is named the "threshold band"), (3) below $80{ }^{\circ} \mathrm{C}$, constant RRA-values of nearly $100 \%$ The threshold band is not highlighted in Fig. 5.8 because insufficient number of data points). The constant RRA-values below $-80{ }^{\circ} \mathrm{C}$ correspond to the lack of the rapid hydrogen diffusion paths through the martensite phase. This interpretation is well supported by the lower RRA found in thermal pre-charged steels. Consequently, in case of the steels with $\mathrm{M}_{\mathrm{d} 30}$ below the threshold band, RRA obtained by tensile tests in a high pressure hydrogen atmosphere is "quasi-static".

\section{The influence of heat treatment}

Some data points are located away from the trend line in RRA vs $\mathrm{M}_{\mathrm{d} 30}$ and attributed to the macro-segregation and variation in grain-size (Fig. 5.8 Fig. 5.10). This trend was found also in the reference steel of W11 (Fig. 5.10). The difference in heat treatment time prior to the tensile test caused large scatter in RRA for W11. Shorter heat treatment time (15 min) resulted in the improvement of its resistance to HE. This impact of heat treatment time is attributed to the change in microstructural properties such as grain size, dislocation density, dislocation structure, and also the type of the oxide layer.

Concerning the grain size and dislocation effects, they are regarded as of minor to HE, as considered from the summary on reference steel W11 in below. 


\section{- Grain size}

Grain size effects are considered to be marginal for W11 since the average grain size in W11 for $15 \mathrm{~min}$ and $30 \mathrm{~min}$ is almost identical. (section $5.2 .3 .1)$

\section{- Dislocation density and dislocation structure}

The dislocation density in W11 annealed for 15 min was one order of magnitude higher than that in W11 annealed for $30 \mathrm{~min}$ (Table 4.3). According to the HELP theory, hydrogen facilitates dislocation motion due to the elastic interaction between $\mathrm{H}$ and dislocations, leading to $\mathrm{HE}$ of steels. Higher dislocation density and higher RRA observed in W11 treated for 15 min is, therefore, inconsistent with the assumption based on the HELP theory.

TEM observation on thinned W11 revealed partial dislocation tangles in W11 annealed for $15 \mathrm{~min}$, while planar dislocations were present in W11 annealed for $30 \mathrm{~min}$ (Fig. 4.18). This variation in the dislocation structure was relatively small compared with that in the literature data which supports the impediment of the dislocation motion model. Thus, for the scatter of W11 for $15 \mathrm{~min}$ and $30 \mathrm{~min}$, it is judged that dislocation structures formed prior to the tensile tests do not play a beneficial role in resistance to HE.

After consideration of the different variables, in a second step the impact of the oxide layers on HE was evaluated

\section{- Oxide layer stacking sequence}

SIMS analysis was performed on the studied alloys to characterize the oxide layer formed in the solution annealing process. A layer stacking of Fe-, $\mathrm{Cr}^{-}$and $\mathrm{Ni}^{-}$-oxide was found. The sequence starting at the surface is Fe-rich oxide, Cr-rich oxide, and Ni-rich oxide. The stacking sequence of $\mathrm{Fe}^{-}$and Cr-oxides was successfully explained by the different ion mobilities in the frame of the oxidation kinetics (section 5.1.5). However, the Ni-oxide does not 
follow this general result and is, from thermodynamics aspects, also the less stable oxide.

To develop a general picture on the oxides contribution to HE valid for all the different steels of this study, the shapes of the oxide layer depth profiles were taken for classification. Four different shapes of oxide layer depth profiles could be deduced: (a) thick oxide, (b) decaying sub-surface oxide, (c) thin surface oxide, and (d) high constant oxide level.

For the reference steel (W11) annealed for $15 \mathrm{~min}$ and $30 \mathrm{~min}$, two distinct shapes were found in the depth profiles; the "high constant oxide level" in 15 min sample and "decaying sub-surface oxide" in 30 min sample, resulting from the difference in the solution annealing time (Fig. 4.16). The "high constant oxide level" layer of W11 for $15 \mathrm{~min}$ is partly due to the local enrichment of oxides as observed in secondary ion images (Fig. 4.17). On one hand, the local enrichment of oxide also has the potential to inhibit the crack propagation process, since macroscopic defects (grain boundaries, segregation bands) work as obstacles. On the other hand, in the microscopic observation, dislocation motion is impeded at obstacles and dislocation pile-up occurs, resulting in the stress concentrations at these sites. Consequently, more research is required to elucidate the crack propagation behavior at obstacles, including oxides, in both macroscopic and microscopic scales.

For the low-nickel steels, all four types of oxide layer shapes were found; (a) thick oxide on W14 and W19, (b) decaying sub-surface oxide on W11, W16, W20 and W21, (c) thin surface oxide on W15 and W18, and (d) high constant oxide level on W12 and W17 (Fig. 4.21).

The RRA-values of most samples follow the linear dependency in the figure of RRA vs $\mathrm{M}_{\mathrm{d} 30}$ (Fig. 5.8) and their accessibility to HE is, therefore, dominated by the martensite contribution. But, RRA for W12 and W17, which possess the layer of high constant oxide level, shows a higher value than the trend line (Fig. 5.8). Importantly, this trend is the same as that observed on W11 for 15 min, suggesting an improvement of the steels' HE 
resistance by this special type of the oxide layer (Fig. 4.16 and Fig. 5.10). High constant oxide continuing in the range from $600 \mathrm{~nm}$ to $1 \mathrm{\mu m}$ in depth may suppress the hydrogen entry at the early stage of tensile testing, because of the slow specimen elongation of $1.65 \mu \mathrm{m} \cdot \mathrm{s}^{-1}$. The relatively high RRA-value found for W19 whose oxide layer is categorized into "thick oxide" is attributed not to the oxide layer type, but to its phase stability.

Cnsequently, main conclusions on the HE-resistance of low-nickel austenitic stainless steels drawn from this study can be briefly listed as follows.

- HE of austenitic stainless steels is primarily governed by the steels' phase stability, since the martensite phase acts as the rapid diffusion path for hydrogen.

- The influence of phase stability distinctly appears in the results of tensile tests in hydrogen atmosphere.

- The effect of the oxide layer on HE depends on the oxide depth profile. For the (a) thick oxide, (b) decaying sub-surface oxide, (c) thin surface oxide types, the oxide layer play a secondary role on HE.

- The oxide layer type (d) of "high constant oxide" improves the resistance of alloys to $\mathrm{HE}$. This was interpreted by the process of crack propagation at early stages in the tensile tests.

As a final remark on the entire aspects reviewed so far, the empirical equation of $\mathrm{M}_{\mathrm{d} 30}$ temperature by Nohara should be noted as beneficial to compare $\mathrm{HE}$ data reported by other groups. Application of this type of examination will be of great help to interpret consistently experimental results on $\mathrm{HE}$ behavior, which involves a large number of variables. On such purpose, more data have to be accumulated from high pressure hydrogen tensile tests. From the data obtained so far, it was clearly shown that, the contributions to HE behavior from macro segregation, grain size, dislocation density and dislocation structure, and oxide layer should be carefully 
examined. Especially, reports regarding the characterization of oxide layer formed during solution annealing and its impact on HE have apparently not been published to date. In this study, it was firstly shown, by using TOF-SIMS, to link the oxide layer properties and their impacts on HE. Further experimental studies, or new experimental techniques that enable to observe a direct association to the mechanical behavior of the oxide layer and its effect on $\mathrm{HE}$, will remain to be an exciting topic. 


\section{Bibliography}

[1] F. Cell, E. Vehicles, A portfolio of power-trains for Europe: a fact-based analysis, (2011).

[2] S. Bakker, H. van Lente, M.T.H. Meeus, Credible expectations - The US Department of Energy's Hydrogen Program as enactor and selector of hydrogen technologies, Technol. Forecast. Soc. Change. 79 (2012) 1059-1071.

[3] H. Vehoff, Hydrogen in Metals III, Bd. 73 der Topics in Applied Physics., Springer Berlin Heidelberg, Berlin, 1997.

[4] C. Borchers, T. Michler, A. Pundt, Effect of Hydrogen on the Mechanical Properties of Stainless Steels, Adv. Eng. Mater. 10 (2008) $11-23$.

[5] W.T. Chandler, R.J. Walter, Effects of High-Pressure Hydrogen on Metals at Ambient Temperature, NASA-CR-102425, R-7780-1, Rocket. Calif. (1969).

[6] S. Fukuyama, L. Zhang, K. Yokogawa, Development of Materials Testing Equipment in High Pressure Hydrogen and Hydrogen Environment Embrittlement of Austenitic Stainless Steels (in Japanese), J. Japan Inst. Met. 68 (2004) 62-65.

[7] Y. Hosoya, A. Inoue, T. Masumoto, Effect of Hydrogen on Crack Cracks Propagation Behavior and Microstructures around Cracks in Austenitic Stainless Steels (in Japanese), Tetsu to Hagane. 64 (1978) 769.

[8] D. Eliezer, D.G. Chakrapani, C.J. Altstetter, E.N. Pugh, The influence of austenite stability on the hydrogen embrittlement and stresscorrosion cracking of stainless steel, Metall. Trans. A. 10 (1979) 935941.

[9] S. Singh, C.J. Altstetter, Effects of hydrogen concentration on slow crack growth in stainless steels, Metall. Trans. A. 13 (1982) 1799-1808.

[10] R.J. Walter, P.R. Jewitt, W.T. Chandler, On the mechanism of hydrogen-environment embrittlement of iron- and nickel-base alloys, Mater. Sci. Eng. 5 (1970) 99-110.

[11] R.J. Walter, W.T. Chandler, Influence of hydrogen pressure and notch severity on hydrogen-environment embrittlement at ambient temperatures, Mater. Sci. Eng. 8 (1971) 90-97. 
[12] G. Schuster, C.J. Altstetter, Fatigue of stainless steel in hydrogen, Metall. Trans. A. 14 (1983) 2085-2090.

[13] T.P. Perng, C.J. Altstetter, Comparison of hydrogen gas embrittlement of austenitic and ferritic stainless steels, Metall. Trans. A. 18 (1987) $123-134$.

[14] T.P. Perng, C.J. Altstetter, Cracking kinetics of two-phase stainless steel alloys in hydrogen gas, Metall. Trans. A. 19 (1988) 145-152.

[15] G. Han, Effect of strain-induced martensite on hydrogen environment embrittlement of sensitized austenitic stainless steels at low temperatures, Acta Mater. 46 (1998) 4559-4570.

[16] M. Martín, S. Weber, C. Izawa, S. Wagner, A. Pundt, W. Theisen, Influence of machining-induced martensite on hydrogen-assisted fracture of AISI type 304 austenitic stainless steel, Int. J. Hydrogen Energy. 36 (2011) 11195-11206.

[17] T. Michler, C. San Marchi, J. Naumann, S. Weber, M. Martín, Hydrogen environment embrittlement of stable austenitic steels, Int. J. Hydrogen Energy. 37 (2012) 16231-16246.

[18] T. Michler, A. Yukhimchuk, J. Naumann, Hydrogen environment embrittlement testing at low temperatures and high pressures, Corros. Sci. 50 (2008) 3519-3526.

[19] H.H. Uchida, E. Fromm, Kinetics of hydrogen absorption of titanium/metal (vanadium, chromium, manganese, iron, nickel) sandwich films with and without oxygen precoverage at $300 \mathrm{~K}$, J. Less Common Met. 131 (1987) 125-132.

[20] M. Martín, Development of lean alloyed austenitic stainless steels with a high resistance to hydrogen environment embrittlement, $\mathrm{PhD}$ thesis, Eigenverlag des Lehrstuhls Werkstofftechnik der Ruhr-Universität Bochum, ISBN: 978-3-943063-09-7, 2012.

[21] H. Kaesche, Corrosion of Metals - Physicochemical Principles and Current Problems, Springer-Verlag, Berlin Heidelberg, New York, 2003.

[22] R. Kirchheim, B. Heine, H. Fischmeister, S. Hofmann, H. Knote, U. Stolz, The passivity of iron-chromium alloys, Corros. Sci. 29 (1989) 899-917.

[23] J.R. Davis, (Ed.), Alloy Digest Sourcebook: Stainless Steels, ASM International, Materials Park, Ohio, 2000. 
[24] M. Hasegawa, (Ed.), Stainless steel Handbook (in Japanese), Nikkan Kogyo Shimbun Co.,Ltd., Tokyo, 1960.

[25] P. Marshall, Austenitic Stainless Steels: Microstructure and mechanical properties, Elsevier applied science publishers, London and New York, 1984.

[26] A.L. Schaeffler, Constitution Diagram for Stainless-steel Weld Metal. 2. Schaeffler Diagram, Met. Prog. 106 (1974) 227-227.

[27] D. Olson, Prediction of austenitic weld metal microstructure and properties, Weld. J. Res. Suppl. (1985) 281s.

[28] M. Kikuchi, T. Maki, T. Sakuma, H. Sudo, I. Tamura, R. Tanaka, Iron and Steel Materials (in Japanese), The Japan Institute of Metals and Materials, Sendai, Japan, 1985.

[29] L. Vitos, P. Korzhavyi, B. Johansson, Elastic Property Maps of Austenitic Stainless Steels, Phys. Rev. Lett. 88 (2002) 155501.

[30] L. Vitos, P. a Korzhavyi, B. Johansson, Stainless steel optimization from quantum mechanical calculations, Nat. Mater. 2 (2003) 25-28.

[31] Z. Nishiyama, Martensite transformation (in Japanese), Maruzen Ltd., Tokyo, 1971.

[32] G.E. Totten, (Ed.), Steel Heat Treatment: Metallurgy and Technologies Steel Heat Treatment Handbook, Second Edition, Taylor \& Francis, Boca Raton, London, New York, 2007.

[33] A.H. Bott, F.B. Pickering, G.J. Butterworth, Development of high manganese high nitrogen low activation austenitic stainless steels, J. Nucl. Mater. 141-143 (1986) 1088-1096.

[34] U. Krupp, C. West, H.-J. Christ, Deformation-induced martensite formation during cyclic deformation of metastable austenitic steel: Influence of temperature and carbon content, Mater. Sci. Eng. A. 481-482 (2008) 713-717.

[35] T.J. Angel, Formation of martensite in austenitic stainless steels, J. Iron Steel Inst. 177 (1954) 165.

[36] K. Nohara, Y. Ono, N. Ohashi, Composition and Grain Size Dependencies of Strain-induced Martensitic Transformation in Metastable Austenitic Stainless Steels (in Japanese), Tetsu to Hagane. 63 (1977) 772-782. 
[37] S.K. Varma, J. Kalyanam, L.E. Murk, V. Srinivas, Effect of grain size on deformation-induced martensite formation in 304 and 316 stainless steels during room temperature tensile testing, J. Mater. Sci. Lett. 13 (1994) 107-111.

[38] K. Kamachi, M. Toge, T. Nakanori, X-Ray Study of Hydrides in Autenitic Stainless Steel (in Japanese), Soc. Mater. Sci. Japan. 26 (1977) 322.

[39] N. Narita, C.J. Altstetter, H.K. Birnbaum, Hydrogen-related phase transformations in austenitic stainless steels, Metall. Trans. A. 13 (1982) 1355-1365.

[40] P. Rozenak, L. Zevin, D. Eliezer, Hydrogen effects on phase transformations in austenitic stainless steels, J. Mater. Sci. 19 (1984) $567-573$.

[41] A.P. Bentley, G.C. Smith, Phase transformation of austenitic stainless steels as a result of cathodic hydrogen charging, Metall. Trans. A. 17 (1986) 1593-1600.

[42] P. Rozenak, D. Eliezer, Phase changes related to hydrogen-induced cracking in austenitic stainless steel, Acta Metall. 35 (1987) 23292340 .

[43] D.G. Ulmer, C.J. Altstetter, Phase relations in the hydrogen-austenite system, Acta Metall. Mater. 41 (1993) 2235-2241.

[44] S. Sugiyama, H. Ohkubo, M. Takenaka, K. Ohsawa, M.I. Ansari, N. Tsukuda, et al., The effect of electrical hydrogen charging on the strength of 316 stainless steel, J. Nucl. Mater. 283-287 (2000) 863-867.

[45] M. Hoelzel, S. a. Danilkin, H. Ehrenberg, D.M. Toebbens, T.J. Udovic, H. Fuess, et al., Effects of high-pressure hydrogen charging on the structure of austenitic stainless steels, Mater. Sci. Eng. A. 384 (2004) $255-261$.

[46] R.A. Oriani, Whitney Award Lecture-1987: Hydrogen-The Versatile Embrittler, Corrosion. 43 (1987) 390-397.

[47] ASTM, Hydrogen Embrittlement Testing, ASTM International, 100 Barr Harbor Drive, PO Box C700, West Conshohocken, PA 19428-2959, 1974.

[48] M.R. Louthan, M.J. Morgan, Some technology gaps in the detection and prediction of hydrogen-induced degradation of metals and alloys, J. Nondestruct. Eval. 15 (1996) 113-120. 
[49] R. Kirchheim, A. Pundt, Hydrogen in Metals, in: D. Laughlin, K. Hono (Eds.), Phys. Metall. Vol. 3, Elsevier, Amsterdam, Waltham, Heidelberg, London, New York, Oxford, Paris, San Diego, San Francisco, Sydney, Tokyo, 2014: pp. 2597-2705.

[50] A. Barnoush, Hydrogen embrittlement, revisited by in situ electrochemical nanoindentation, $\mathrm{PhD}$ thesis, Universität des Saarlandes, 2007.

[51] F. Terasaki, N. Takano, Mechanism of Hydrogen Embrittlement in Iron and Low Strength Steels (in Japanese), Mater. Japan. 33 (1994) 922.

[52] S.P. Lynch, Metallographic and fractographic techniques for characterising and understanding hydrogen-assisted cracking of metals, in: R.P. Gangloff, B.P. Somerday (Eds.), Gaseous Hydrog. Embrittlement Mater. Energy Technol. Vol. 1 Probl. Its Characterisation Eff. Part. Alloy Classes, Woodhead Publishing Ltd., 80 High Street, Sawston, Cambridge, CB22 3HJ, UK, 2012.

[53] J.P. Hirth, Effects of hydrogen on the properties of iron and steel, Metall. Trans. A. 11 (1980) 861-890.

[54] C.D. Beachem, A new model for hydrogen-assisted cracking (hydrogen "embrittlement"), Metall. Trans. 3 (1972) 441-455.

[55] S.P. Lynch, Environmentally assisted cracking: Overview of evidence for an adsorption-induced localised-slip process, Acta Metall. 36 (1988) 2639-2661.

[56] H.K. Birnbaum, P. Sofronis, Hydrogen-enhanced localized plasticity - a mechanism for hydrogen-related fracture, Mater. Sci. Eng. A. 176 (1994) 191-202.

[57] J. Morlet, H. Johson, A.R. Troiano, A new concept of hydrogen embrittlement in steel, J. Iron Steel Inst. 189 (1958) 37.

[58] A.R. Troiano, The role of hydrogen and other interstitials in the mechanical behavior of metals, Trans. Metall. Soc. AIME. 52 (1960) 54.

[59] W. Whiteman, A.R. Troiano, Hydrogen embrittlement of austenitic stainless steel, Corrosion. 21 (1965) 53.

[60] R.A. Oriani, The diffusion and trapping of hydrogen in steel, Acta Metall. 18 (1970) 147-157. 
[61] H.A. Wriedt, R.A. Oriani, Effect of tensile and compressive elastic stress on equilibrium hydrogen solubility in a solid, Acta Metall. 18 (1970) 753-760.

[62] R.A. Oriani, P.H. Josephic, Equilibrium aspects of hydrogen-induced cracking of steels, Acta Metall. 22 (1974) 1065-1074.

[63] R.A. Oriani, P.H. Josephic, Hydrogen-enhanced load relaxation in a deformed medium־carbon steel, Acta Metall. 27 (1979) 997-1005.

[64] R.A. Oriani, P.H. Josephic, Equilibrium and kinetic studies of the hydrogen-assisted cracking of steel, Acta Metall. 25 (1977) 979-988.

[65] T. Matsumoto, J. Eastman, H.K. Birnbaum, Direct observations of enhanced dislocation mobility due to hydrogen, Scr. Metall. 15 (1981) 1033-1037.

[66] A. Kimura, H.K. Birnbaum, Plastic softening by hydrogen plasma charging in pure iron, Scr. Metall. 21 (1987) 53-57.

[67] I.M. Robertson, H.K. Birnbaum, An HVEM study of hydrogen effects on the deformation and fracture of nickel, Acta Metall. 34 (1986) 353366 .

[68] I.M. Robertson, D. Tetter, Controlled environment transmission electron microscopy., Microsc. Res. Tech. 42 (1998) 260-9.

[69] P.J. Ferreira, I.M. Robertson, H.K. Birnbaum, Hydrogen effects on the interaction between dislocations, Acta Mater. 46 (1998) 1749-1757.

[70] P.J. Ferreira, I.M. Robertson, H.K. Birnbaum, Hydrogen effects on the character of dislocations in high-purity aluminum, Acta Mater. 47 (1999) 2991-2998.

[71] I.M. Robertson, The effect of hydrogen on dislocation dynamics, Eng. Fract. Mech. 68 (2001) 671-692.

[72] D.F. Teter, I.M. Robertson, H.K. Birnbaum, The effects of hydrogen on the deformation and fracture of B-titanium, Acta Mater. 49 (2001) 4313-4323.

[73] T. Tabata, H.K. Birnbaum, Direct observations of hydrogen enhanced crack propagation in iron, Scr. Metall. 18 (1984) 231-236.

[74] P. Rozenak, I.M. Robertson, H.K. Birnbaum, HVEM studies of the effects of hydrogen on the deformation and fracture of AISI type 316 austenitic stainless steel, Acta Metall. Mater. 38 (1990) 2031-2040. 
[75] P. Sofronis, H.K. Birnbaum, Mechanics of the hydrogen - dislocation impurity interactions-I. Increasing shear modulus, J. Mech. Phys. Solids. 43 (1995) 49-90.

[76] R. Kirchheim, Reducing grain boundary, dislocation line and vacancy formation energies by solute segregation. I. Theoretical background, Acta Mater. 55 (2007) 5129-5138.

[77] R. Kirchheim, Revisiting hydrogen embrittlement models and hydrogen-induced homogeneous nucleation of dislocations, Scr. Mater. 62 (2010) 67-70.

[78] R. Kirchheim, Reducing grain boundary, dislocation line and vacancy formation energies by solute segregationII. Experimental evidence and consequences, Acta Mater. 55 (2007) 5139-5148.

[79] S. Pillot, L. Coudreuse, Hydrogen-induced disbonding and embrittlement of steels used in petrochemical refining, in: R.P. Gangloff, B.P. Somerday (Eds.), Gaseous Hydrog. Embrittlement Mater. Energy Technol. Vol. 1 Probl. Its Characterisation Eff. Part. Alloy Classes, Woodhead Publishing Ltd., 2012.

[80] P.R. Jewitt, R.J. Walter, W.T. Chandler, R.P. Frohmberg, Hydrogen Environment Embrittlement of Metals (NASA CR-2163), NASA CR-2163. (1973).

[81] C. San Marchi, B.P. Somerday, Technical Reference on Hydrogen Compatibility of Materials, SANDIA REPORT, SAND2008-1163. (2008).

[82] T. Michler, M. Lindner, U. Eberle, J. Meusinger, Assessing hydrogen embrittlement in automotive hydrogen tanks, in: R.P. Gangloff, B.P. Somerday (Eds.), Gaseous Hydrog. Embrittlement Mater. Energy Technol. Vol. 1 Probl. Its Characterisation Eff. Part. Alloy Classes, Woodhead Publishing Ltd., 2012.

[83] R.J. Walter, W.T. Chandler, Influence of gaseous hydrogen on metals, NASA-CR-124410. (1973).

[84] H.R. Gray, Hydrogen Environment Embrittle, NASA-TM-X-68088; E-6995, Rocketdyne for the National Aeronautics and Space Administration, Canoga Park CA, 1972.

[85] Linde, Hydrogen 6.0, http://www.linde.com/.

[86] H. Mehrer, Springer Series in Splid-State Sciences 155, Diffusion in Solid: Fundamentals, Methods, Materials, Diffusion-Controlled Processes, Springer-Verlag, Berlin, Heidelberg, New York, 2007. 
[87] J.K. Gorman, W.R. Nardella, Hydrogen permeation through metals, Vacuum. 12 (1962) 19-24.

[88] C. San Marchi, B. Somerday, S. Robinson, Permeability, solubility and diffusivity of hydrogen isotopes in stainless steels at high gas pressures, Int. J. Hydrogen Energy. 32 (2007) 100-116.

[89] British Stainless Steel Association, http://www.bssa.org.uk/.

[90] A.D. Le Claire, Permeation of Gases through Solids, Defect Diffus. Forum. 34 (1983) 1.

[91] G. Alefeld, J. Völkl, Hydrogen in Metals I, Bd. 28 der Topics in Applied Physics., Springer-Verlag, Berlin, Heidelberg, New York, 1978.

[92] T. Tanabe, Y. Yamanishi, K. Sawada, S. Imoto, Hydrogen transport in stainless steels, J. Nucl. Mater. 123 (1984) 1568-1572.

[93] N. Birks, G.H. Meier, F.S. Pettit, Introduction to the high-temperature oxidation of metals, 2nd Ed., Cambridge University Press, The Edinburgh Building, Cambridge CB2 8RU, UK, 2006.

[94] S.R.J. Saunders, J.R. Nicholls, Oxidation, Hot Corrosion and Protection of Metallic Materials, in: R.W. Cahn, P. Haasen (Eds.), Phys. Metall. vol.2, 4 th, Elsevier Science B. V., Amsterdam, Lausanne, New York, Oxford, Shannon, Tokyo, 1996.

[95] J.R. Davis, (Ed.), ASM Specialty Handbook: Heat-Resistant Materials, ASM International, Ohio, 1997.

[96] C.O. A Olsson, D. Landolt, Passive films on stainless steels - Chemistry, structure and growth, Electrochim. Acta. 48 (2003) 1093-1104.

[97] A. Atkinson, R.I. Taylor, Impurity diffusion in $\mathrm{NiO}$ grain boundaries, J. Phys. Chem. Solids. 47 (1986) 315-323.

[98] TWI Ltd., http://www.twi-global.com/.

[99] Under great pressure - TWI's Hydrogen test facility, http://www.youtube.com/.

[100] G142-98 (2004), Standard test method for determination of susceptibility of metals to embrittlement in hydrogen containing environments at high pressure, high temperature, or both., ASTM International, West Conshohocken, 2004. 
[101] A. Benninghoven, The History of Static SIMS: A Personal Perspective, in: J.C. Vickerman, D. Briggs (Eds.), ToF-SIMS Surf. Anal. by Mass Spectrom., Chichester, IM Publications, 2001: pp. 41-72.

[102] D. Rading, TOF-SIMS 5, Product Presentation, ION-TOF User's Meet. Shin-Osaka, Japan. (2005).

[103] ION-TOF GmbH, TOF-SIMS Software ver.4.1.

[104] T. Grehl, Improvement in TOF-SIMS Instrumentation for Analytical Application and Fundamental Research, PhD Thesis, Westfälische Wilhelms-Universitat Münster, 2003.

[105] H.A. Storms, K.F. Brown, J.D. Stein, Evaluation of a cesium positive ion source for secondary ion mass spectrometry, Anal. Chem. 49 (1977) 2023-2030.

[106] K. Asami, K. Hashimoto, An X-ray photo-electron spectroscopic study of surface treatments of stainless steels, Corros. Sci. 19 (1979) 10071017.

[107] D.F. Reich, Sample Handling, in: J.C. Vickerman, D. Briggs (Eds.), ToF-SIMS Surf. Anal. by Mass Spectrom., 1st ed., IM Publications, Chichester UK and SurfaceSpectra, Manchester UK, 2001: pp. 113135.

[108] JEOL, Guide to Scanning Microsocope Observation, http://www.jeolusa.com/.

[109] A. Putnis, An Introduction to Mineral Sciences, Cambridge University Press, 1992.

[110] J. Thomas, T. Gemming, Analytical Transmission Electron Microscopy, Springer Netherlands, Dordrecht, Heidelberg, New York, London, 2014.

[111] B.D. Cullity, Elements of X-Ray Diffraction 2nd ed., Addison-Wesley publishing company, 1978.

[112] G. Williamson, W. Hall, X-ray line broadening from filed aluminium and wolfram, Acta Metall. 1 (1953) 22-31.

[113] G.K. Williamson, R.E. Smallman, III. Dislocation densities in some annealed and cold-worked metals from measurements on the X-ray debye-scherrer spectrum, Philos. Mag. 1 (1956) 34-46.

[114] Struers A/S, Metallographic preparation of stainless steel, http://www.struers.com/ 
[115] P.J. Gellings, M.A. DE Jongh, Grain boundary oxidation and the chromium-depletion theory of intercrystalline corrosion of austenitic stainless steels, Corros. Sci. 7 (1967) 413-421.

[116] E.A. Gulbransen, K.F. Andrew, F.A. Brassart, Oxidation of Molybdenum $550{ }^{\circ} \mathrm{C}$ to $1700{ }^{\circ} \mathrm{C}$, J. Electrochem. Soc. 110 (1963) 952959.

[117] B.D. Bastow, D.P. Whittle, G.C. Wood, Alloy depletion profiles resulting from the preferential removal of the less noble metal during alloy oxidation, Oxid. Met. 12 (1978) 413-438.

[118] A.C.S. Sabioni, R.P.B. Ramos, V. Ji, F. Jomard, W.A.D.A. Macedo, P.L. Gastelois, et al., About the Role of Chromium and Oxygen Ion Diffusion on the Growth Mechanism of Oxidation Films of the AISI 304 Austenitic Stainless Steel, Oxid. Met. 78 (2012) 211-220.

[119] A.C.S. Sabioni, R.P.B. Ramos, J. Vincent, F.J. Jomard, F.Emiliane Advíncula Malheiros, Vincent Ji, Oxygen Diffusion Study in Oxidation Films of the AISI 304 Austenitic Stainless Steel, Defect Diffus. Forum. 323 (2012) 345.

[120] A.F. Padilha, R.L. Plaut, P.R. Rios, Annealing of Cold-worked Austenitic Stainless Steels., ISIJ Int. 43 (2003) 135-143.

[121] R.E. Smallman, R.J. Bishop, Modern Physical Metallurgy and Materials Engineering: Science, Process, Applications, Butterworth-Heinemann, Oxford, 1999.

[122] F.H. Stott, G.C. Wood, J. Stringer, The influence of alloying elements on the development and maintenance of protective scales, Oxid. Met. 44 (1995) 113-145.

[123] G.B. Olson, M. Cohen, Kinetics of strain-induced martensitic nucleation, Metall. Trans. A. 6 (1975) 791-795.

[124] G. Betz, Composition-vs-depth profiles obtained with Auger electron spectroscopy of air-oxidized stainless-steel surfaces, J. Appl. Phys. 45 (1974) 5312.

[125] R. Schubert, The analysis of 301 stainless steel by SIMS, J. Vac. Sci. Technol. 12 (1975) 505.

[126] T. Tanabe, S. Imoto, Surface Oxidation of Type 316 Stainless Steel, Trans. Japan Inst. Met. 20 (1979) 507.

[127] I. Olefjord, The passive state of stainless steels, Mater. Sci. Eng. 42 (1980) 161-171. 
[128] D.R. Baer, Protective and non-protective oxide formation on 304 stainless steel, Appl. Surf. Sci. 7 (1981) 69-82.

[129] D.R. Baer, M.D. Merz, Differences in oxides on large-and small-grained 304 stainless steel, Metall. Trans. A. 11 (1980) 1973-1980.

[130]G.J. Stokkers, A. van Silfhout, G.A. Bootsma, T. Fransen, P.J. Gellings, Interaction of oxygen with an AISI 314 stainless steel surface studied by ellipsometry and auger electron spectroscopy in combination with ion bombardment, Corros. Sci. 23 (1983) 195-204.

[131] J.C. Langevoort, L.J. Hanekamp, P.J. Gellings, On the kinetics of oxidation of austenitic stainless steels AISI 304 and incoloy 800H, Appl. Surf. Sci. 28 (1987) 189-203.

[132] G.C. Allen, J.M. Dyke, S.J. Harris, A. Morris, A surface study of the oxidation of type 304L stainless steel at $600 \mathrm{~K}$ in air, Oxid. Met. 29 (1988) 391-408.

[133] S. Shibagaki, A. Koga, Y. Shirakawa, H. Onishi, H. Yokokawa, J. Tanaka, Chemical reaction path for thin film oxidation of stainless steel, Thin Solid Films. 303 (1997) 101-106.

[134] A. Vesel, M. Mozetič, A. Zalar, Oxidation of AISI 304L stainless steel surface with atomic oxygen, Appl. Surf. Sci. 200 (2002) 94-103.

[135] A. Vesel, M. Mozetic, A. Drenik, N. Hauptman, M. Balat-Pichelin, High temperature oxidation of stainless steel AISI316L in air plasma, Appl. Surf. Sci. 255 (2008) 1759-1765.

[136] A.N. Hansson, M. Mogensen, S. Linderoth, M.A.. Somers, Inter-Diffusion between $\mathrm{NiO}$ Coating and the Oxide Scale on Fe-22Cr Alloy, J. Corros. Sci. Eng. 6 (2003) 73.

[137] I. Saeki, T. Saito, R. Furuichi, H. Konno, T. Nakamura, K. Mabuchi, et al., Growth process of protective oxides formed on type 304 and 430 stainless steels at 1273 k, Corros. Sci. 40 (1998) 1295-1302.

[138] N. V. Bangaru, Diffusion coatings of steels: Formation mechanism and microstructure of aluminized heat-resistant stainless steels, J. Vac. Sci. Technol. B Microelectron. Nanom. Struct. 2 (1984) 806.

[139] R. Hales, The high temperature oxidation behaviour of austenitic stainless steels, Mater. Corros. Und Korrosion. 29 (1978) 393-399.

[140] R.E. Lobnig, H.P. Schmidt, K. Hennesen, H.J. Grabke, Diffusion of cations in chromia layers grown on iron-base alloys, Oxid. Met. 37 (1992) 81-93. 
[141] F.H. Stott, F.I. Wei, C.A. Enahoro, The influence of manganese on the High-temperature oxidation of iron-chromium alloys, Mater. Corros. 40 (1989) 198-205.

[142] G.C. Wood, High-temperature oxidation of alloys, Oxid. Met. 2 (1970) $11-57$.

[143] J.D. Hodge, Diffusion of Chromium in Magnetite as a Function of Oxygen Partial Pressure, J. Electrochem. Soc. 125 (1978) 55C.

[144] R.K. Wild, Vacuum annealing of stainless steel at temperatures between 770 and 1470K, Corros. Sci. 14 (1974) 575-586.

[145] N.S. McIntyre, T.C. Chan, C. Chen, Characterization of oxide structures formed on nickel-chromium alloy during low pressure oxidation at $500-600{ }^{\circ} \mathrm{C}$, Oxid. Met. 33 (1990) 457-479.

[146] G.C. Allen, J.M. Dyke, S.J. Harris, A. Morris, The oxidation of Inconel-690 alloy at $600 \mathrm{~K}$ in air, Appl. Surf. Sci. 31 (1988) 220-238.

[147] H.J. Mathieu, D. Landolt, An investigation of thin oxide films thermally grown in situ on $\mathrm{Fe}-24 \mathrm{Cr}$ and $\mathrm{Fe}-24 \mathrm{Cr}-11 \mathrm{Mo}$ by auger electron spectroscopy and X-ray photoelectron spectroscopy, Corros. Sci. 26 (1986) 547-559.

[148] W.E. Boggs, The Oxidation of Iron-Aluminum Alloys from $450{ }^{\circ} \mathrm{C}$ to $900{ }^{\circ} \mathrm{C}$, J. Electrochem. Soc. 118 (1971) 906-913.

[149] H. Ike, H. Okabe, E. Tsuji, The Oxidation Behavior of Fe-14Cr Alloys with Low Al Contents at High Temperatures, J. Japan Inst. Met. (in Japanese). 42 (1978) 509-517.

[150] J.C. Pivin, D. Delaunay, C. Roques-Carmes, A.M. Huntz, P. Lacombe, Oxidation mechanism of $\mathrm{Fe}-\mathrm{Ni}-20-25 \mathrm{Cr}-5 \mathrm{Al}$ alloys-influence of small amounts of yttrium on oxidation kinetics and oxide adherence, Corros. Sci. 20 (1980) 351-373.

[151] V. Ramakrishnan, J. A. McGurty, N. Jayaraman, Oxidation of high-aluminum austenitic stainless steels, Oxid. Met. 30 (1988) 185200 .

[152]R. Prescott, M.J. Graham, The oxidation of iron-aluminum alloys, Oxid. Met. 38 (1992) 73-87.

[153] Y. Yamamoto, M.P. Brady, Z.P. Lu, P.J. Maziasz, C.T. Liu, B.A. Pint, et al., Creep-resistant, Al2O3-forming austenitic stainless steels., Science. 316 (2007) 433-6. 
[154] M.P. Brady, Y. Yamamoto, M.L. Santella, P.J. Maziasz, B.A. Pint, C.T. Liu, et al., The development of alumina-forming austenitic stainless steels for high-temperature structural use, JOM. 60 (2008) 12-18.

[155] B. Predel, Landolt-Börnstein, Group IV: Physical Chemistry, Thermodynamic Properties: Phase Equilibria, Crystallographic and Thermodynamic Data of Binary Alloys . Ac-Au - Au-Zr, Springer-Verlag, Berlin/Heidelberg, 1991.

[156] L.M. Atlas, W.K. Sumida, Solidus, Subsolidus, and Subdissociation Phase Equilibria in the System Fe-Al-O, J. Am. Ceram. Soc. 41 (1958) 150-160.

[157] M.G.C. Cox, B. Mcenaney, V.D. Scott, A chemical diffusion model for partitioning of transition elements in oxide scales on alloys, Philos. Mag. 26 (1972) 839-851.

[158] J.D. Dunitz, L.E. Orgel, Electronic properties of transition-metal oxides-II, J. Phys. Chem. Solids. 3 (1957) 318-323.

[159] Y. Mine, C. Narazaki, K. Murakami, S. Matsuoka, Y. Murakami, Hydrogen transport in solution-treated and pre-strained austenitic stainless steels and its role in hydrogen-enhanced fatigue crack growth, Int. J. Hydrogen Energy. 34 (2009) 1097-1107.

[160] S.S. Hecker, M.G. Stout, K.P. Staudhammer, J.L. Smith, Effects of Strain State and Strain Rate on Deformation-Induced Transformation in 304 Stainless Steel: Part I. Magnetic Measurements and Mechanical Behavior, Metall. Trans. A. 13 (1982) 619-626.

[161] L.E. Murr, K.P. Staudhammer, S.S. Hecker, Effects of Strain State and Strain Rate on Deformation-Induced Transformation in 304 Stainless Steel: Part II. Microstructural Study, Metall. Trans. A. 13 (1982) 627635 .

[162] J. Talonen, H. Hänninen, Formation of shear bands and strain-induced martensite during plastic deformation of metastable austenitic stainless steels, Acta Mater. 55 (2007) 6108-6118.

[163] J. Talonen, H. Hänninen, P. Nenonen, G. Pape, Effect of strain rate on the strain-induced $\mathrm{Y} \rightarrow \mathrm{a}^{\prime}$-martensite transformation and mechanical properties of austenitic stainless steels, Metall. Mater. Trans. A. 36 (2005) 421-432.

[164] L. Zhang, M. Wen, M. Imade, S. Fukuyama, K. Yokogawa, Effect of nickel equivalent on hydrogen gas embrittlement of austenitic stainless steels based on type 316 at low temperatures, Acta Mater. 56 (2008) 3414-3421. 
[165] C. San Marchi, B.P. Somerday, X. Tang, G.H. Schiroky, Effects of alloy composition and strain hardening on tensile fracture of hydrogen-precharged type 316 stainless steels, Int. J. Hydrogen Energy. 33 (2008) 889-904.

[166] G.B. Olson, M. Cohen, A mechanism for the strain-induced nucleation of martensitic transformations, J. Less Common Met. 28 (1972) 107118.

[167] L. Bracke, L. Kestens, J. Penning, Transformation mechanism of $\mathrm{a}^{\prime}$-martensite in an austenitic Fe-Mn-C-N alloy, Scr. Mater. 57 (2007) $385-388$.

[168] A.J. Bogers, W.G. Burgers, Partial dislocations on the $\{110\}$ planes in the B.C.C. lattice and the transition of the F.C.C. into the B.C.C. lattice, Acta Metall. 12 (1964) 255-261.

[169] T. Suzuki, H. Kojima, K. Suzuki, T. Hashimoto, M. Ichihara, An experimental study of the martensite nucleation and growth in 18/8 stainless steel, Acta Metall. 25 (1977) 1151-1162.

[170] J.W. Brooks, M.H. Loretto, R.E. Smallman, In situ observations of the formation of martensite in stainless steel, Acta Metall. 27 (1979) 18291838.

[171] T.S. Byun, N. Hashimoto, K. Farrell, Temperature dependence of strain hardening and plastic instability behaviors in austenitic stainless steels, Acta Mater. 52 (2004) 3889-3899.

[172] S. Fukuyama, D. Sun, L. Zhang, M. Wen, K. Yokogawa, Effect of Temperature on Hydrogen Environment Embrittlement of Type 316 Series Austenitic Stainless Seels at Low Temperatures, J. Japan Inst. Met. 67 (2003) 456-459.

[173] S. Hannula, H. Hänninen, S. Tähtinen, Influence of nitrogen alloying on hydrogen embrittlement in AISI 304-type stainless steels, Metall. Trans. A. 15 (1984) 2205-2211.

[174] T.L. Capeletti, M.R. Louthan, The Tensile Ductility of Austenitic Steels in Air and Hydrogen, J. Eng. Mater. Technol. 99 (1977) 153.

[175] M.R. Louthan, G.R. Caskey, J.. Donovan, D.. Rawl, Hydrogen embrittlement of metals, Mater. Sci. Eng. 10 (1972) 357-368.

[176] J.A. Brooks, A.J. West, Hydrogen induced ductility losses in austenitic stainless steel welds, Metall. Trans. A. 12 (1981) 213-223. 
[177] C. San Marchi, B.P. Somerday, X. Tang, G.H. Schiroky, Hydrogen Assisted Fracture of Type 316 Stainless Steel at Sub-Ambient Temperature, in: Proc. PVP2008 2008 ASME Press. Vessel. Pip. Div. Conf. July 27-31, 2008, Chicago, Illinois, USA, ASME, 2008: pp. 191199.

[178] V. Shrinivas, S.K. Varma, L.E. Murr, Deformation-induced martensitic characteristics in 304 and 316 stainless steels during room-temperature rolling, Metall. Mater. Trans. A. 26 (1995) 661-671.

[179] K. Yokogawa, S. Fukuyama, Method for deciding austenite stainless steel, Japan Patent Publication No. 2005-00995, 2005.

[180] H. Fujii, S. Ohmiya, Tensile properties of SUS304 stainless steel in high pressure hydrogen at room temperature, J. High Press. Insitute Japan (in Japanese). 47 (2009) 85-94.

[181] S. Fukuyama, L. Zhang, M. Wen, K. Yokogawa, Tensile Properties of SUS304 Stainless Steel in High Pressure Hydrogen at Room Temperature (in Japanese), J. Japan Inst. Met. 67 (2003) 157-160.

[182] R.J. Walter, W.T. Chandler, Effects of high pressure hydrogen on metals at ambient temperature, NASA Rep. R-7780-1 Prep. by Rocket. Res. Center, Canoga Park. CA, USA. (1969).

[183] G.R. Caskey, Hydrogen compatibility handbook for stainless steels, Savannah River Lab., Tech. Rep. DP-1643. (1983).

[184] S. Weber, M. Martín, W. Theisen, Impact of heat treatment on the mechanical properties of AISI $304 \mathrm{~L}$ austenitic stainless steel in high-pressure hydrogen gas, J. Mater. Sci. 47 (2012) 6095-6107.

[185] G142-98, Standard test method for determination of susceptibility of metals to embrittlement in hydrogen containing environments at high pressure, high temperature, or both., in: ASTM International, West Conshohocken, 1998.

[186] G142-98 (2011), Standard test method for determination of susceptibility of metals to embrittlement in hydrogen containing environments at high pressure, high temperature, or both., in: ASTM International, West Conshohocken, 2011.

[187] N.R. Moody, R.E. Stoltz, M.W. Perra, The effect of hydrogen on fracture toughness of the $\mathrm{Fe}-\mathrm{Ni}$-Co superalloy IN903, Metall. Trans. A. 18 (1987) 1469-1482. 
[188] E. Minkovitz, D. Eliezer, Grain-size and heat-treatment effects in hydrogen-assisted cracking of austenitic stainless steels, J. Mater. Sci. 17 (1982) 3165-3172.

[189] P. Rozenak, D. Eliezer, Effects of metallurgical variables on hydrogen embrittlement in AISI type 316, 321 and 347 stainless steels, Mater. Sci. Eng. 61 (1983) 31-41.

[190] R.W. Cahn, Recovery and Recrystallization, in: R.W. Cahn, P. Haasen (Eds.), Phys. Metall. vol.3, 4 th, Elsevier Science B. V., Amsterdam, Lausanne, New York, Oxford, Shannon, Tokyo, 1996.

[191] A.J. West, M.R. Louthan, Dislocation transport and hydrogen embrittlement, Metall. Trans. A. 10 (1979) 1675-1682.

[192] B. Pieraggi, R.A. Rapp, Stress generation and vacancy annihilation during scale growth limited by cation-vacancy diffusion, Acta Metall. 36 (1988) 1281-1289.

[193] J. Robertson, M.I. Manning, Limits to adherence of oxide scales, Mater. Sci. Technol. 6 (1990) 81.

[194] K.A. Nibur, B.P. Somerday, D.K. Balch, C. San Marchi, The role of localized deformation in hydrogen-assisted crack propagation in $21 \mathrm{Cr}-$ 6Ni-9Mn stainless steel, Acta Mater. 57 (2009) 3795-3809.

[195] M.R. Louthan, J.A. Donovan, G.R. Caskey, Hydrogen diffusion and trapping in nickel, Acta Metall. 23 (1975) 745-749.

[196] P. Tsong-Pyng, C.J. Altstetter, Effects of deformation on hydrogen permeation in austenitic stainless steels, Acta Metall. 34 (1986) 17711781.

[197] S. Xiukui, X. Jian, L. Yiyi, Hydrogen permeation behaviour in austenitic stainless steels, Mater. Sci. Eng. A. 114 (1989) 179-187.

[198] Y. Mine, T. Kimoto, Hydrogen uptake in austenitic stainless steels by exposure to gaseous hydrogen and its effect on tensile deformation, Corros. Sci. 53 (2011) 2619-2629.

[199] H. Katsuta, K. Furukawa, Hydrogen and Deuterium Transport through Type 304 Stainless Steel at Elevated Temperatures, J. Nucl. Sci. Technol. 18 (1981) 143-151.

[200] Y. Sakamoto, H. Katayama, The Electrochemical Determination of Diffusivity and Solubility of Hydrogen in an Austenitic Type 304 Steel (in Japanese), J. Japan Inst. Met. 46 (1982) 805. 
[201] D.M. Grant, D.L. Cummings, D. a. Blackburn, Hydrogen in 304 steel: Diffusion, permeation and surface reaction, J. Nucl. Mater. 149 (1987) 180-191.

[202] T. Shiraishi, M. Nishikawa, T. Yamaguchi, K. Kenmotsu, Permeation of multi-component hydrogen isotopes through austenitic stainless steels, J. Nucl. Mater. 273 (1999) 60-65.

[203] E. Hashimoto, T. Kino, Hydrogen permeation through type 316 stainless steels and ferritic steel for a fusion reactor, J. Nucl. Mater. 133-134 (1985) 289-291.

[204] N. Kishimoto, T. Tanabe, T. Suzuki, H. Yoshida, Hydrogen diffusion and solution at high temperatures in $316 \mathrm{~L}$ stainless steel and nickel-base heat-resistant alloys, J. Nucl. Mater. 127 (1985) 1-9.

[205] D.M. Grant, D.L. Cummings, D.A. Blackburn, Hydrogen in 316 steel diffusion, permeation and surface reaction, J. Nucl. Mater. 152 (1988) $139-145$.

[206] K.S. Forcey, D.K. Ross, J.C.B. Simpson, D.S. Evans, Hydrogen transport and solubility in 316L and 1.4914 steels for fusion reactor applications, J. Nucl. Mater. 160 (1988) 117-124.

[207] S.A. Steward, Review of hydrogen isotope permeability through materials, Lawrence Livermore Natl. Lab, Tech. Rep. UCRL-53441. (1983). 


\section{Appendix}

\subsection{An overview on shared roles}

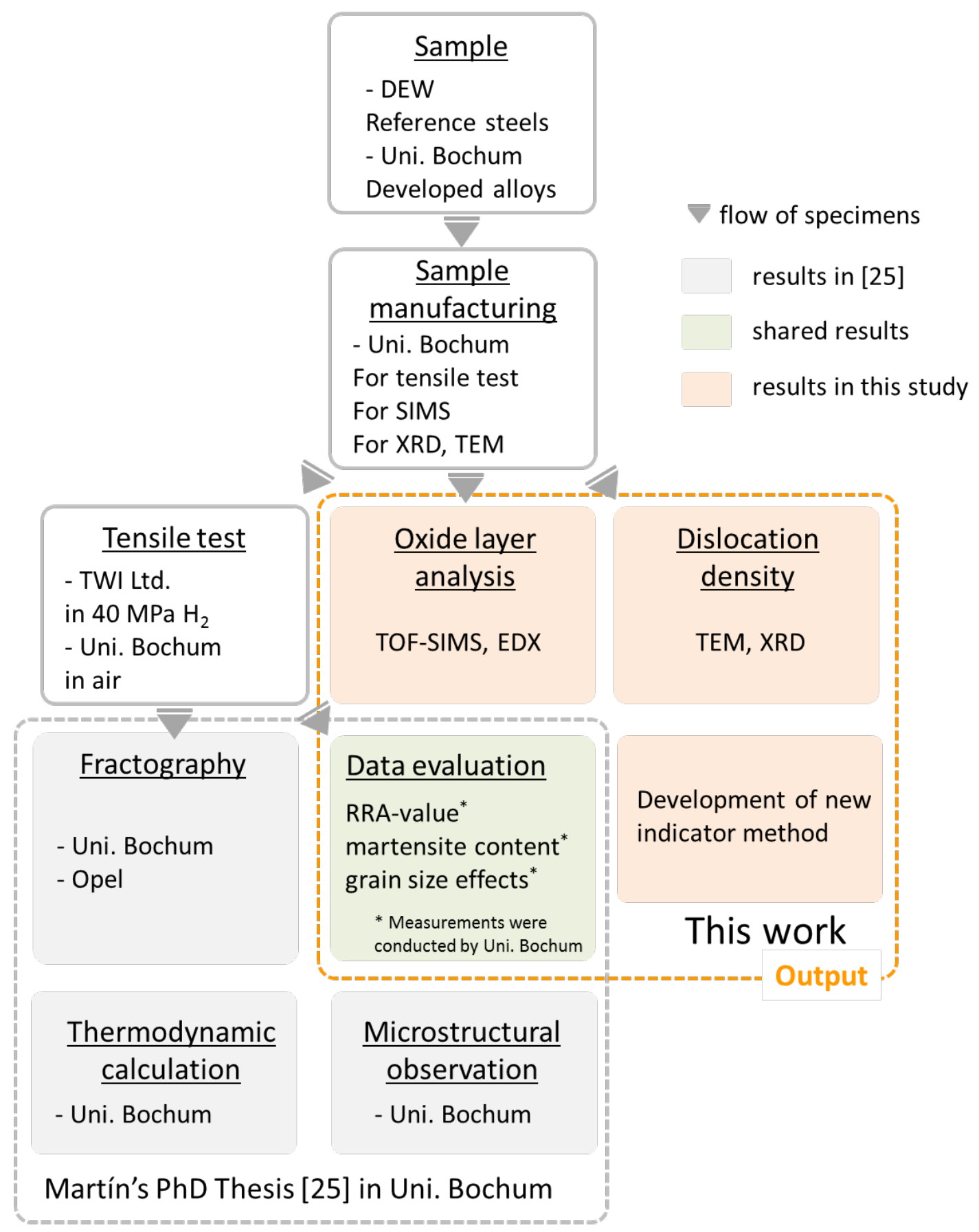




\subsection{Diffusion data}

Table 8.1 Activation parameters of $\mathrm{H}$ permeation in $\mathrm{Ni}, \mathrm{Fe}$ and $\mathrm{Pd}$ according to the data cited in Steward's review [207]

\begin{tabular}{llll}
\hline & Permeability & Reference \\
& \multicolumn{2}{c}{$\Phi=\Phi_{0} \exp \left(-H_{\Phi} / R T\right)$} & \\
& $\Phi_{0}\left(\frac{m o l H_{2}}{m s \sqrt{M P a}}\right)$ & $H_{\Phi}\left(\frac{k J}{m o l}\right)$ & \\
\hline $\mathrm{Ni}$ & $3.22 \times 10^{-4}$ & 54.79 & (Robertson 1973) \\
$\mathrm{Fe}$ & $4.10 \times 10^{-5}$ & 34.92 & (Gonzaletz 1967) \\
$\mathrm{Pd}$ & $2.20 \times 10^{-4}$ & 15.67 & (Koffler 1969) \\
\hline
\end{tabular}

Table 8.2 Activation parameters of $\mathrm{H}$ diffusion in $\mathrm{Ni}$, $\mathrm{Fe}$ and $\mathrm{Pd}$ according to Alefeld and Völkl [91]

\begin{tabular}{lll}
\hline & \multicolumn{2}{c}{$D=D_{0} \exp \left(-H_{D} / R T\right)$} \\
& $D_{0}\left(\frac{\mathrm{m}^{2}}{s}\right)$ & $H_{\mathrm{D}}\left(\frac{\mathrm{kJ}}{\mathrm{mol}}\right)$ \\
& $4.8 \times 10-7$ & 39.3 \\
\hline $\mathrm{Ni}(\mathrm{T}<\mathrm{Tc})$ & $6.9 \times 10-7$ & 40.5 \\
$\mathrm{Ni}(\mathrm{T}>\mathrm{TC})$ & $4.15 \times 10-8$ & 4.2 \\
$\mathrm{Fe}$ & $2.90 \times 10-7$ & 22.2 \\
$\mathrm{Pd}$ &
\end{tabular}




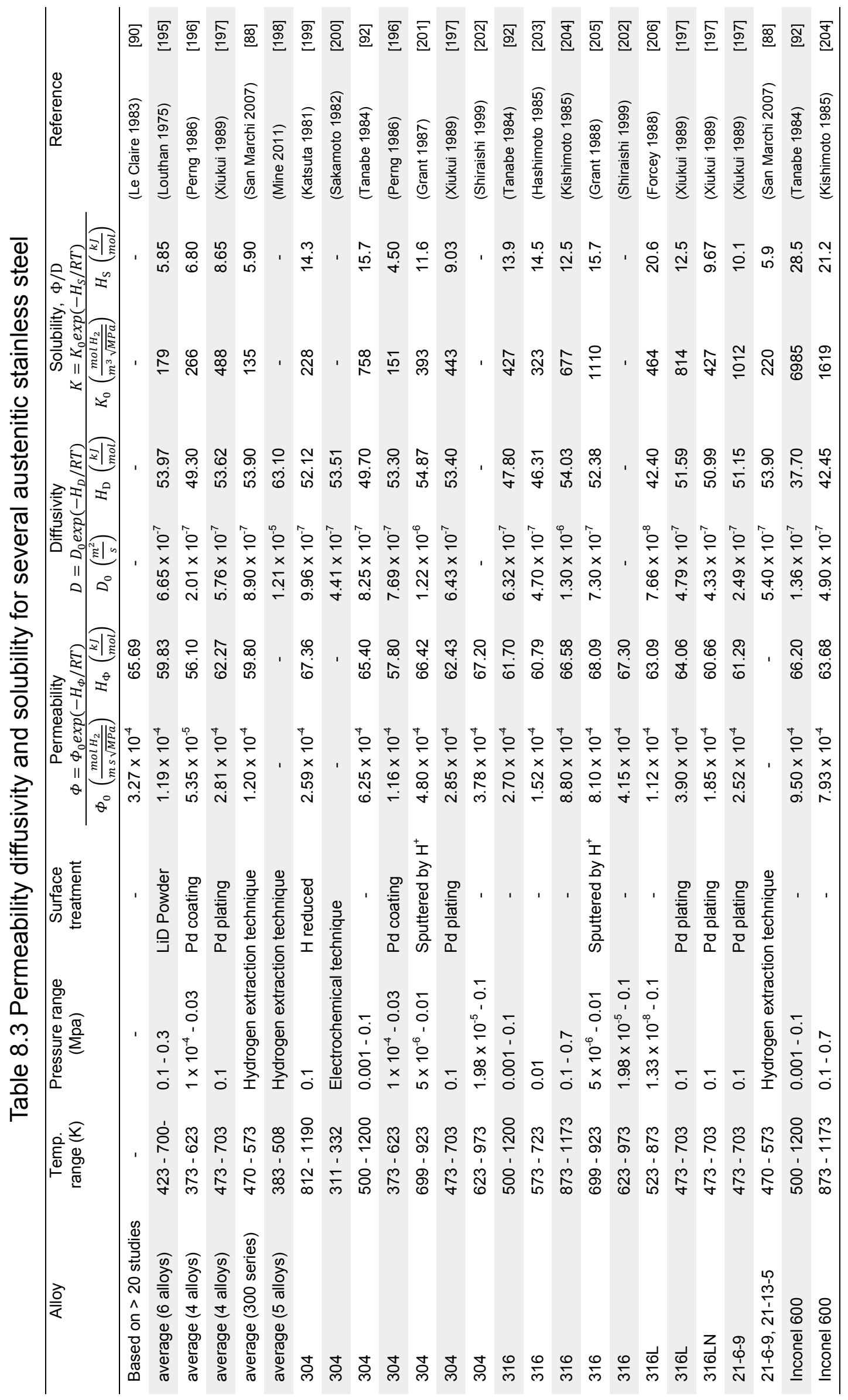




\subsection{Long depth profile on W12 and W17}
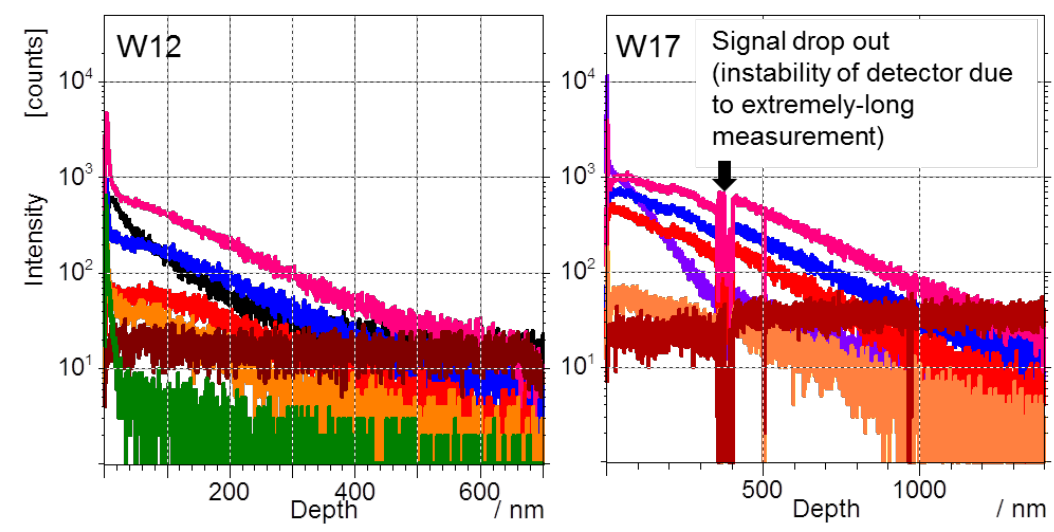


\subsection{Repeatability measurements on W11-W21}

(a) decaying sub-surface oxide: W11, W16, W20, W21
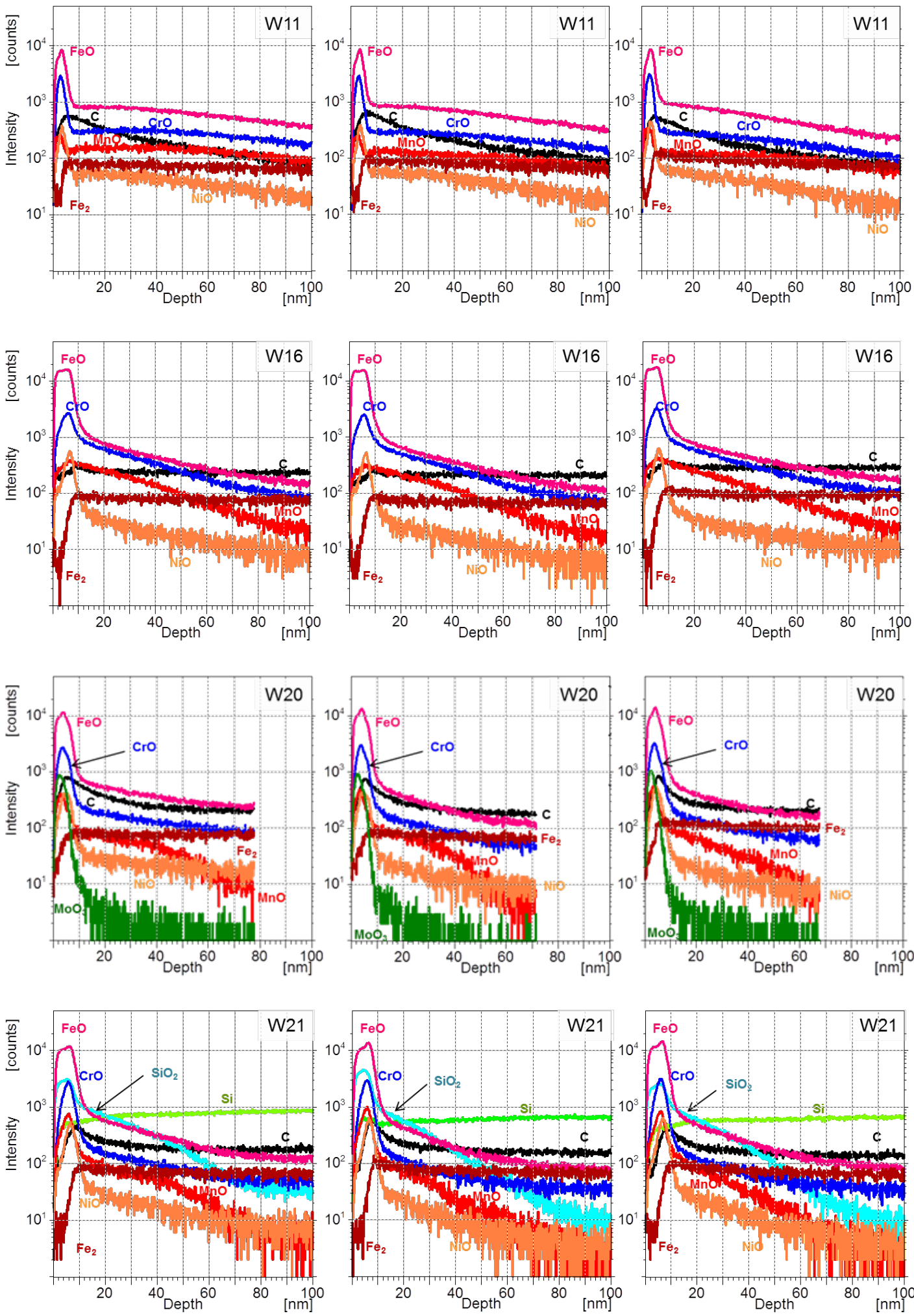

(b) thin surface oxide: W15, W18 

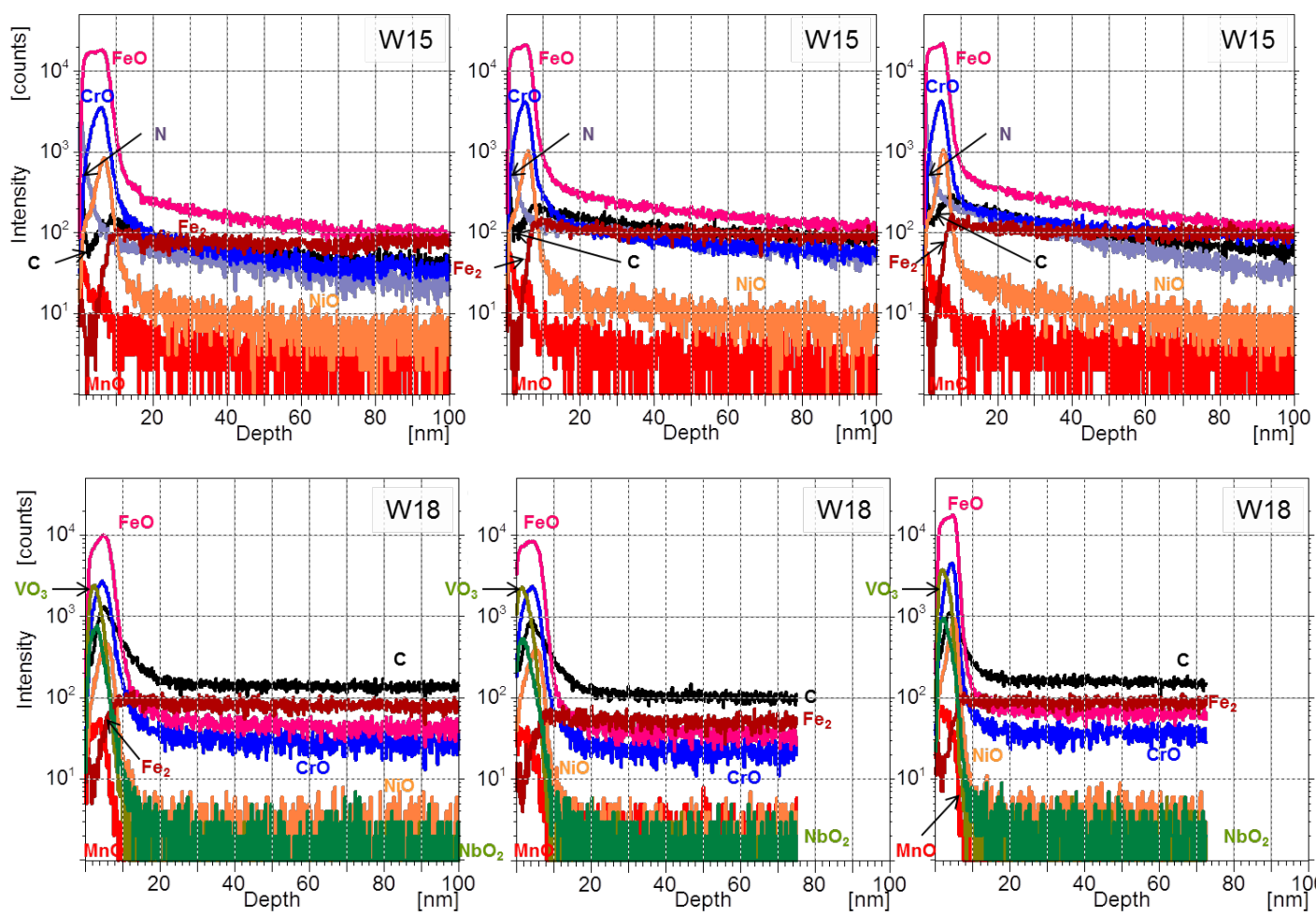

(c) high constant oxide level: W12, W17
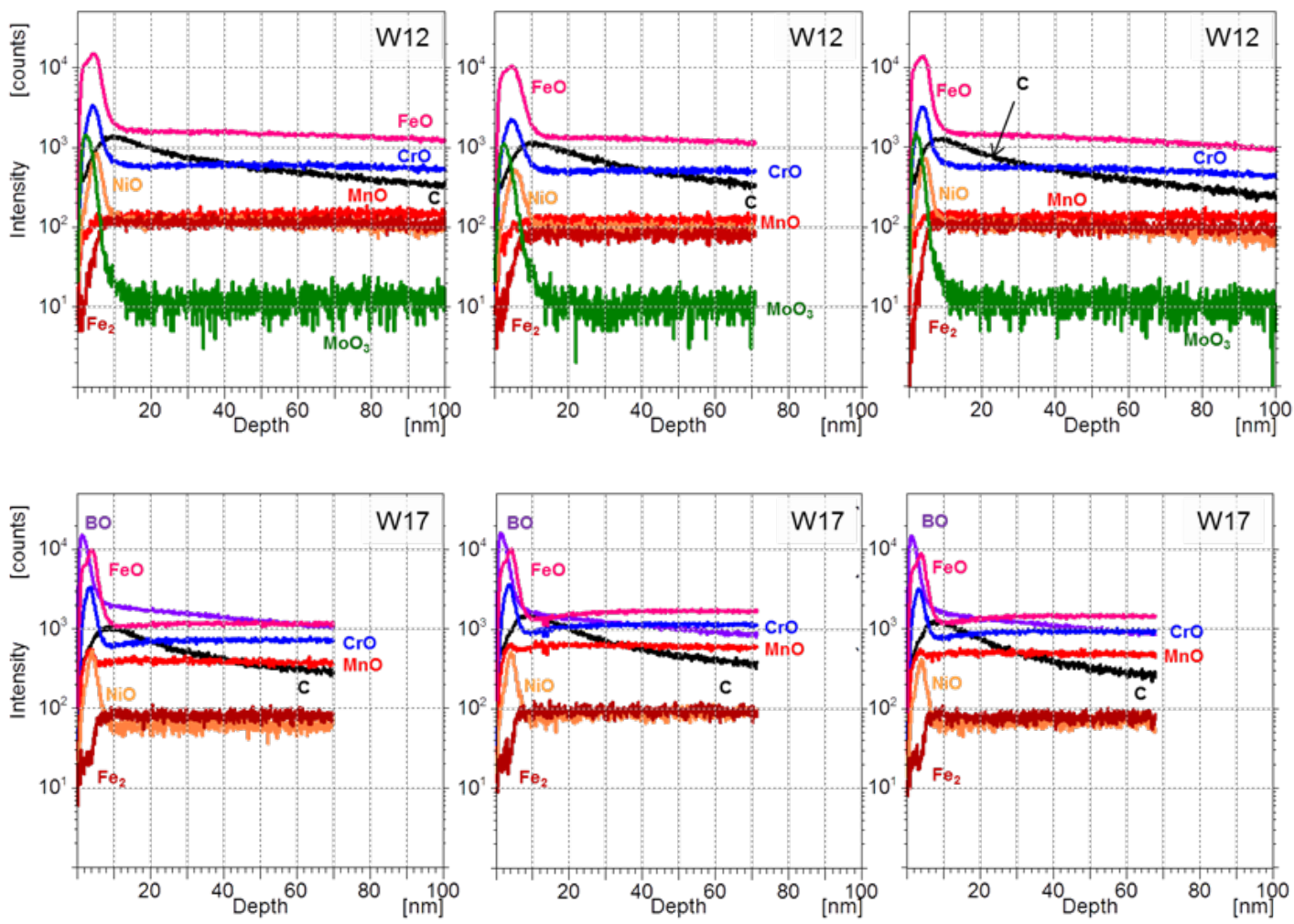


\subsection{Ion images on W11-W21}

The ion images on the low-nickel steels are represented. These images were derived for two different characteristic sample depths of (I) 0-10 nm and (II) $40-50 \mathrm{~nm}$. The depth profile corresponding to these images are shown in Fig. 4.21 and section 8.4.

(a) decaying sub-surface oxide: W11, W16, W20, W21

W11 annealed for 30 min in graphite heater

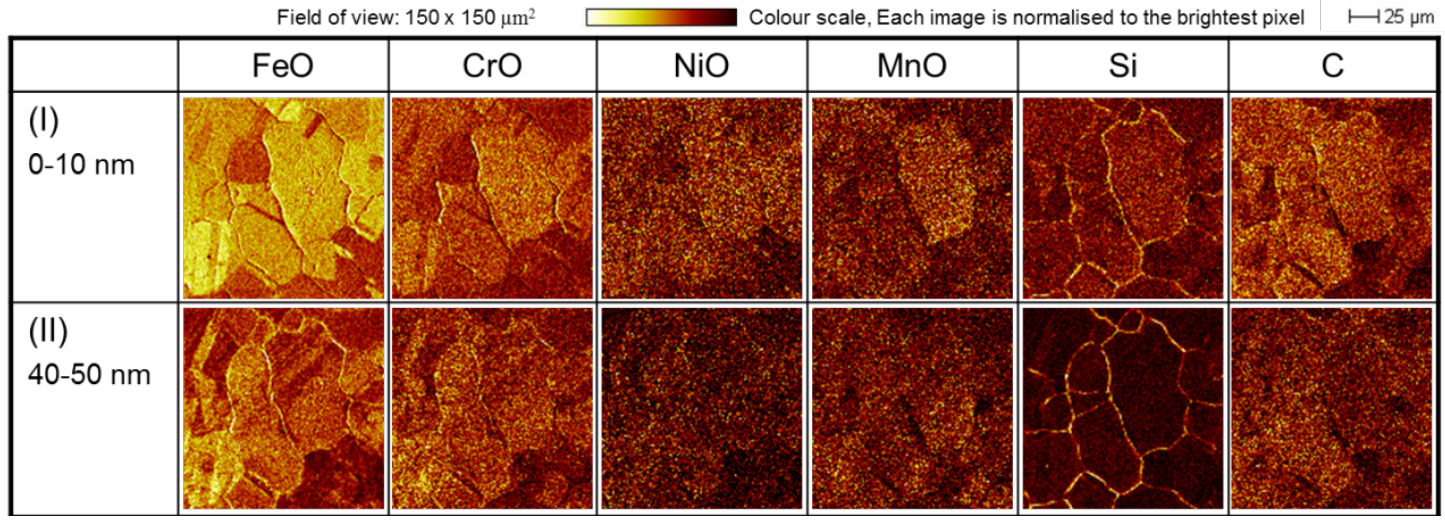

W16 annealed for 30 min in graphite heater

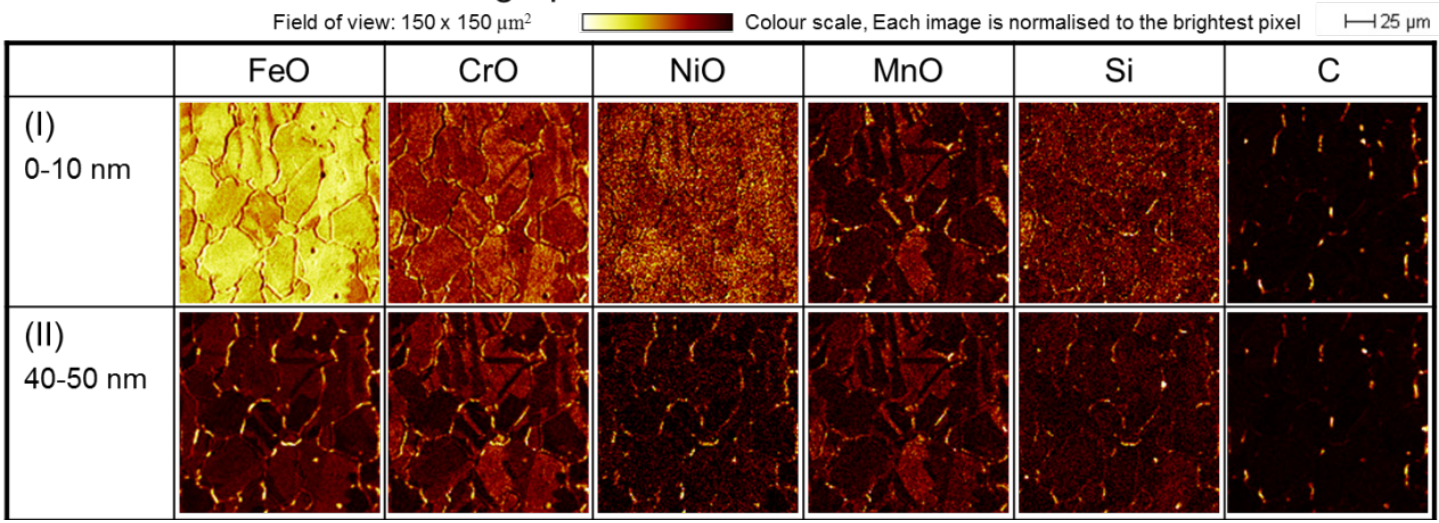

W20 annealed for $30 \mathrm{~min}$ in graphite heater

\begin{tabular}{|c|c|c|c|c|c|c|}
\hline & $\mathrm{FeO}$ & $\mathrm{CrO}$ & $\mathrm{NiO}$ & $\mathrm{MnO}$ & $\mathrm{Si}$ & C \\
\hline $\begin{array}{l}(\mathrm{I}) \\
0-10 \mathrm{~nm}\end{array}$ & & & & & & \\
\hline $\begin{array}{l}\text { (II) } \\
40-50 \mathrm{~nm}\end{array}$ & & & & & & \\
\hline
\end{tabular}


W21 annealed for $30 \mathrm{~min}$ in graphite heater

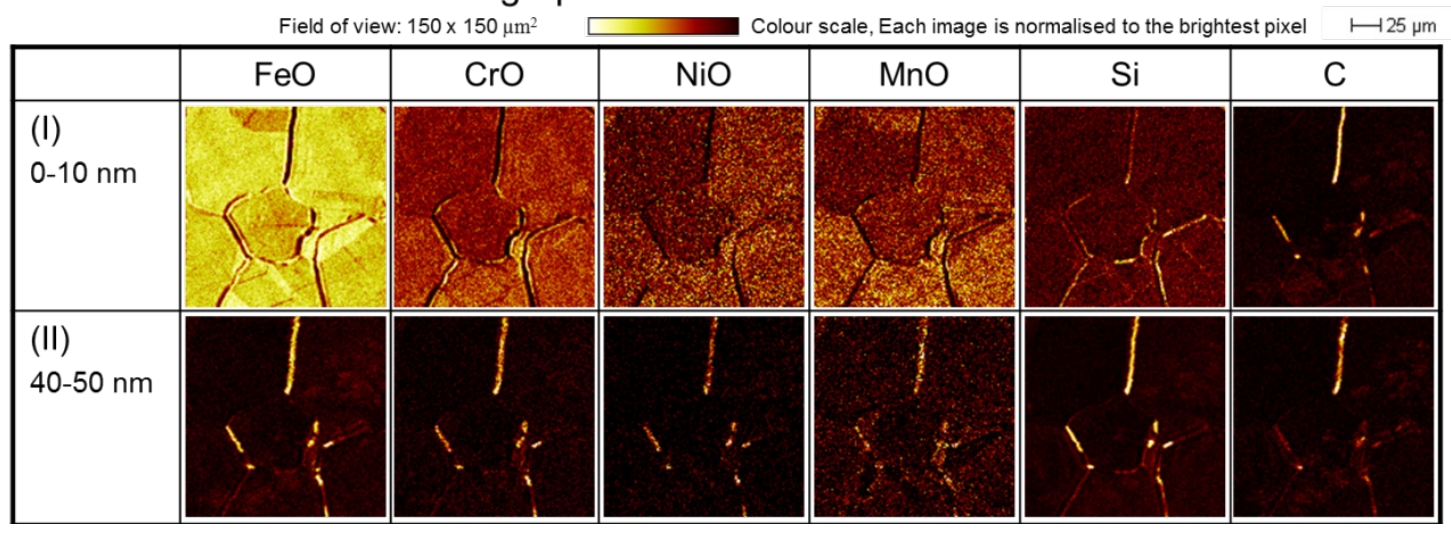

(b) thin surface oxide: W15, W18

W15 annealed for $30 \mathrm{~min}$ in graphite heater

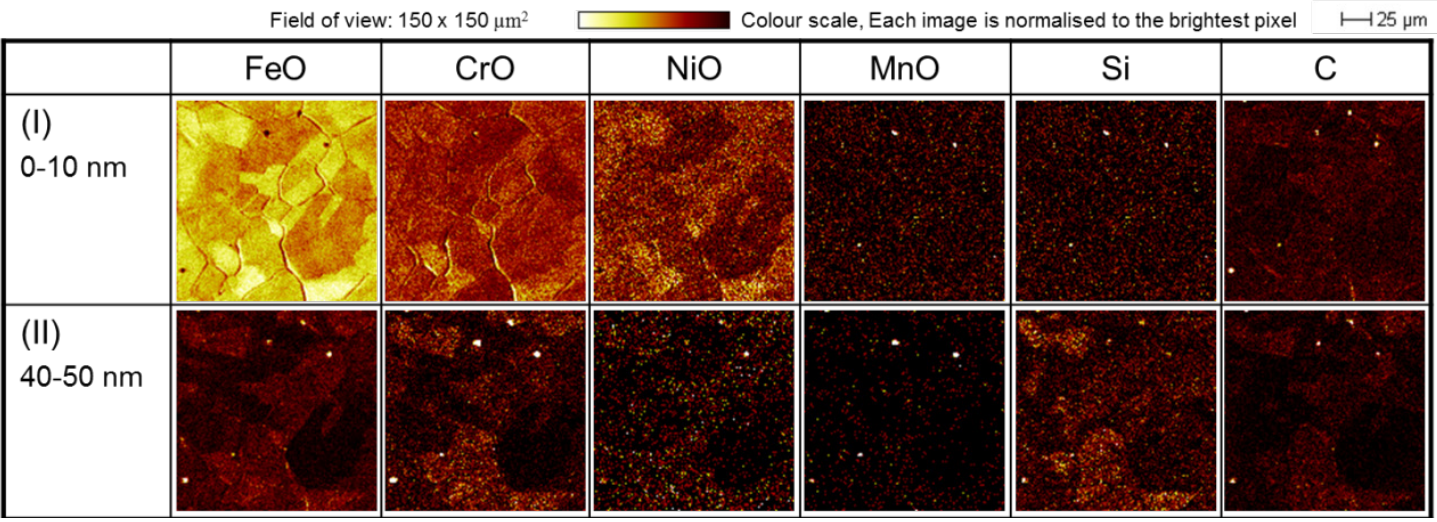

W18 annealed for $30 \mathrm{~min}$ in graphite heater

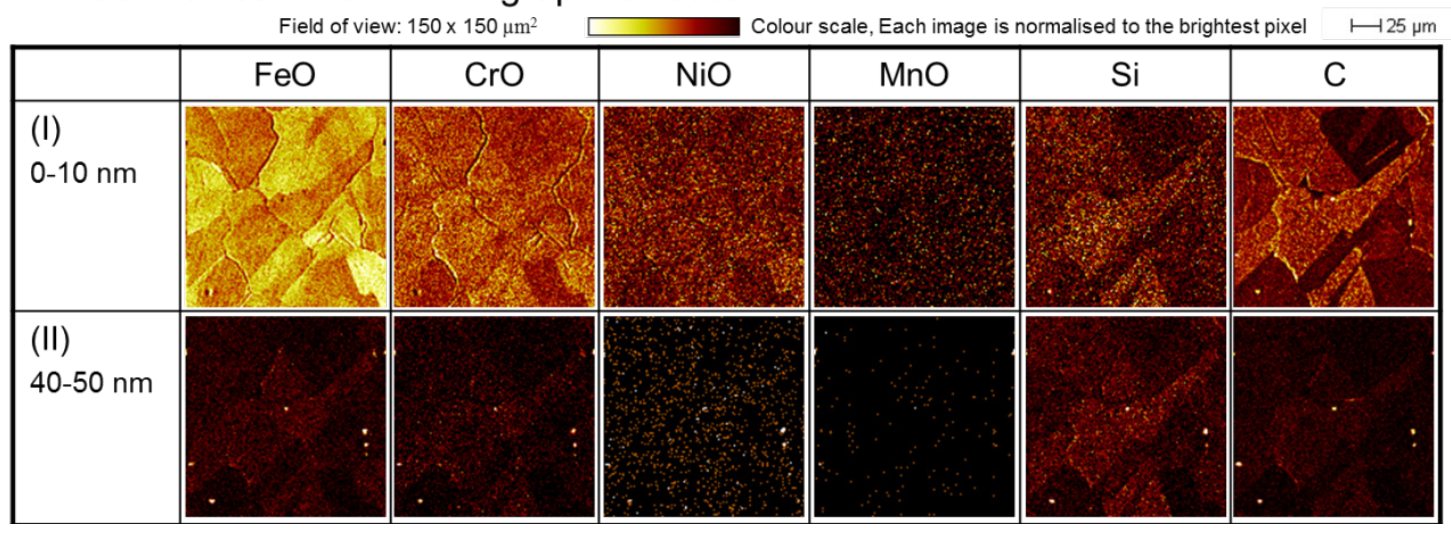


(c) high constant oxide level: W12, W17

W12 annealed for $30 \mathrm{~min}$ in graphite heater

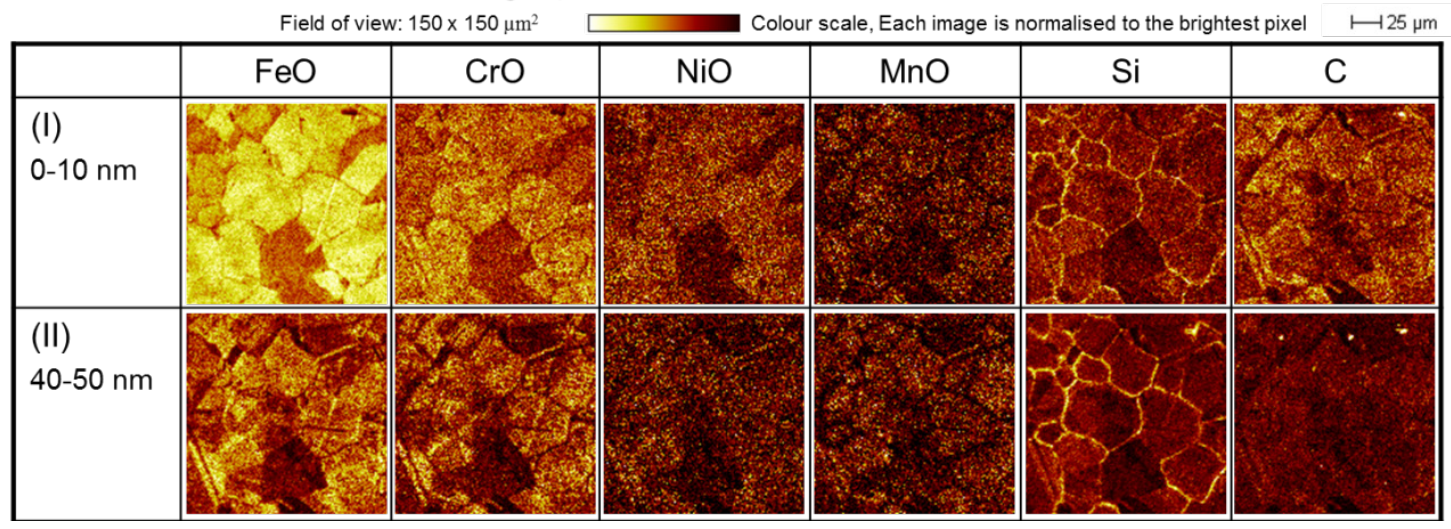

W17 annealed for 30 min in graphite heater

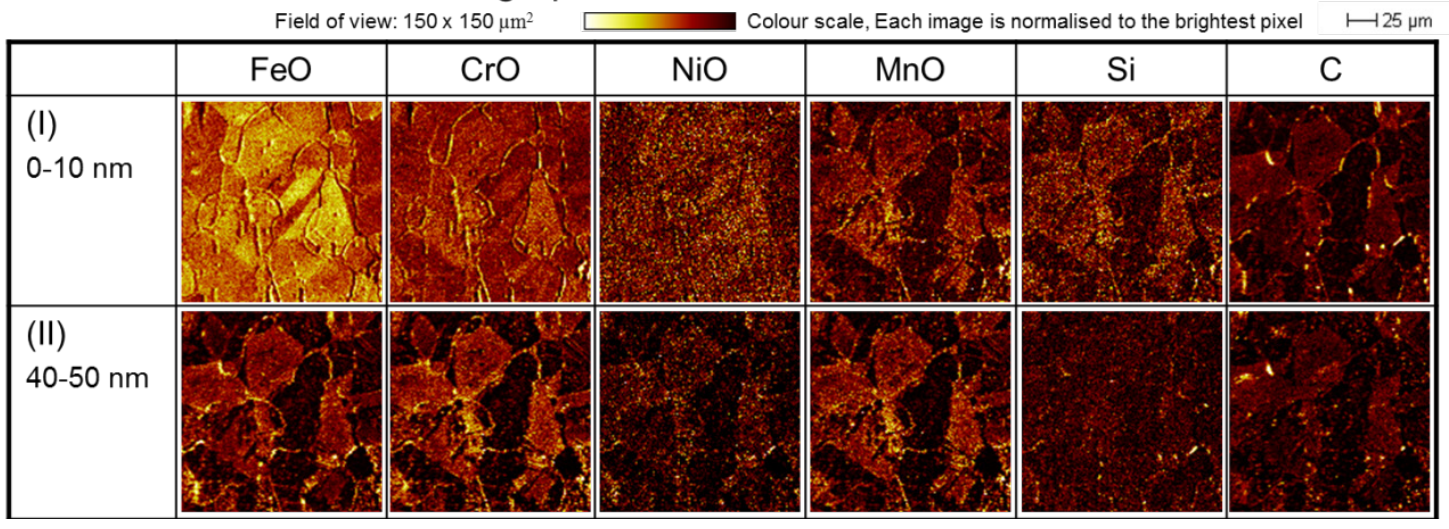




\subsection{Depth profile on Polished W11-W21}
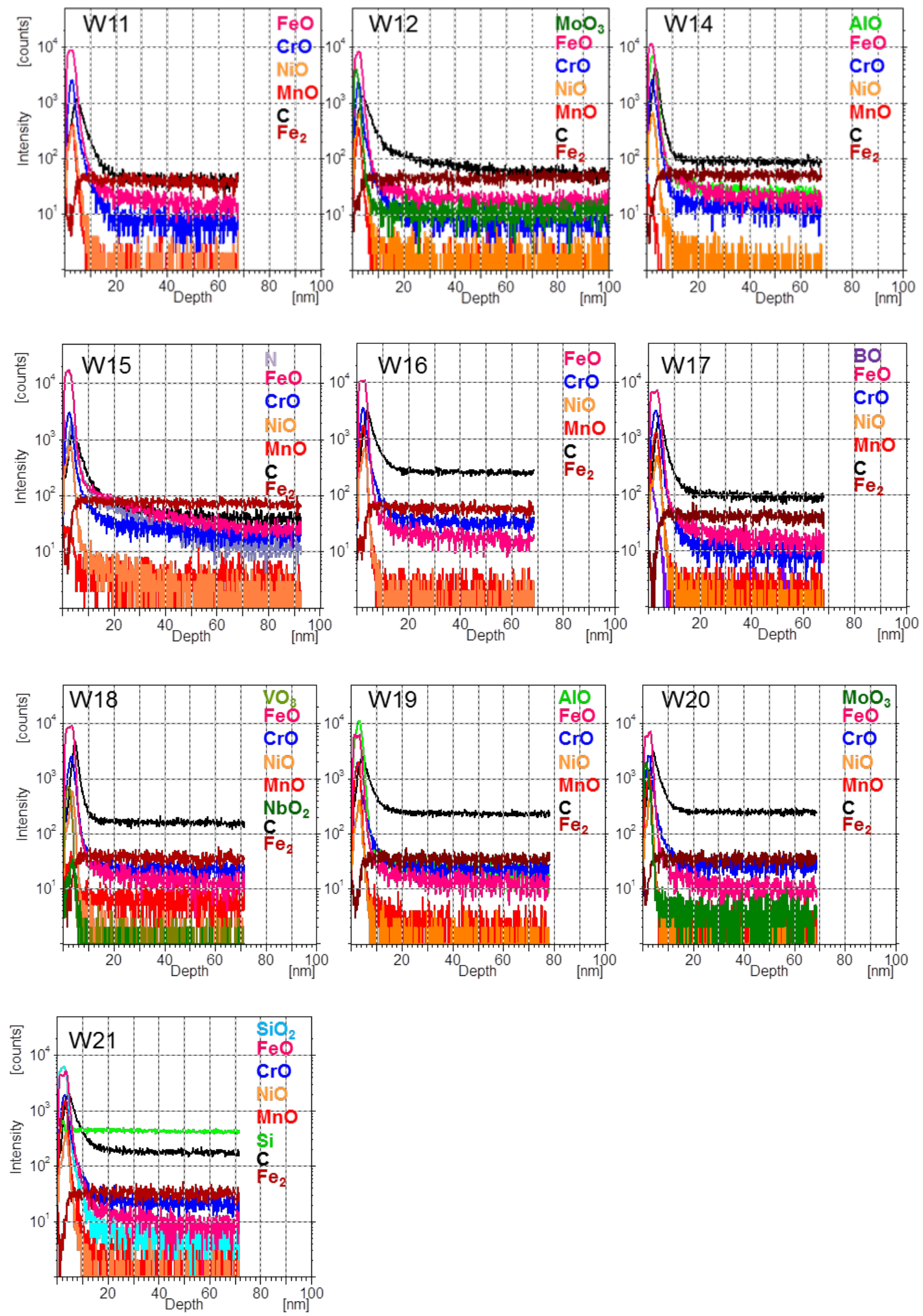


\section{List of publications}

[1] C. Izawa, H.H Uchida, H. Okuhira, Y. Nishi, Hydrogen absorption of $\mathrm{LaNi}_{5}$ after LiOD treatment and surface characterization by TOF-SIMS, Appl. Surf. Sci., 203-204 (2003) 665-668

[2] C. Izawa, S. Wagner, M. Martin, S. Weber, A. Bourgeon, R. Pargeter, T. Michler, A. Pundt, SIMS study on the surface chemistry of stainless steel AISI 304 cylindrical tensile test samples showing hydrogen embrittlement, J. Alloys Comp., 509 (2011) S885-S890

[3] M. Martin, S. Weber, C. Izawa, S. Wagner, A. Pundt, W. Theisen, Influence of machining-induced martensite on hydrogen-assisted fracture of AISI type 304 austenitic stainless steel, Int. J. Hydrogen Energy 36 (2011) 11195-11206

[4] C. Izawa, S. Wagner, M. Martin, S. Weber, R. Pargeter, T. Michler, H.H. Uchida, A. Pundt, SIMS analysis on austenitic stainless steel: The influence of type of oxide surface layer on hydrogen embrittlement, J. Alloys Comp., 580 (2013) S13-S17 


\section{Danksagung}

An erster stelle möchte ich Frau Prof. Dr. Astrid Pundt ganz herzlich danken für die hervorragende Betreuung meiner Arbeit und ihre unerschütterliche Zuversicht und mentale Unterstützung meines Studiums. Ohne ihre freundliche Unterstützung und Ermutigung auch in allen Lebenslagen hätte ich diese Arbeit nicht durchführen können. Ich möchte noch einmal meine tiefe Dankbarkeit dafür ausdrücken.

Auch danke ich Herrn Prof. Dr. Reiner Kirchheim herzlich für seine großzügige Unterstützung meines Promotionsvorhabens in Deutschland, sein stets offenes Ohr zu jeder Zeit, und für die Übernahme des Korreferates.

Den weiteren Mitgliedern der Wasserstoff-in-Metallen-Gruppe, auch den ehemaligen, Dr. Stefan Wagner, Helmut Takahiro Uchida, Vladimir Burlaka, Magnus Hamm, Marian Bongers, Anshu Tyagi und den Mitgliedern der Defactants-Gruppe, Dr. Christine Borchers, Martin Deutges, Marie Christine Tiegel, möchte ich danken.

Ein besonder Dank geht an Dr. Stefan Wagner für seine Unterstützung im HYDEE Projekt.

Marting Deutiges danke ich herzlich für allseitige Kooperation und Beihilfe an der TEM-Beobachtung.

Dieter Plischke, Frank Köhler, Florian Oelkers-Hilker, Tobias Schulz, Denny Wagner, Michael Tetzlaff, Michael Malchow, Cornelia Mewes, Volker Radisch, Matthias Hahn, Karin Ahlborn, Kerstin Born danke ich für Ihre technische Unterstützung.

Insbesondere danke ich Florian Oelkers-Hilker und Kerstin Born für die Probepräparation.

Außerdem danke ich herzlich Matthias Hahn für die Unterstützung bei REM-Messungen.

Ein spezieller Dank geht an Frau Christine Kuba, Frau Karin Haake, Frau Kerstin Reinecke und Frau Phuong Quan für ihre freundliche 
Persönliche Unterstützung bei meines Aufenthalts im Institut und in Deutschland.

Ich danke der ION-TOF GmbH für sorgfältige Unterstützung bei der Wartungs des TOF-SIMS.

Zusätzlich HYDEE Projekt danke ich herzlich dem BMBF und Kooperationspartnern des HYDEE-Projekts für die finanzielle Unterstützung dieser Arbeit und den Mitgliedern von HYDEE für ihre Discussionsbeiträge.

In der Tokai Universität in Japan habe ich großzügige Unterstützung von Prof. Dr. Haru-Hisa Uchida und Herrn Yasuo Miyamoto erfahren. Hier möchte ich meine tiefe Dankbarkeit dafür ausdrücken.

Jedoch geht mein herzlichster Dank an Dr. Ryota Gemma, ohne dessen Unterstützung diese Arbeit nicht möglich gewesen wäre.

Nicht zuletzt danke ich ganz herzlich meinen Vater im Himmel, und meiner Mutter und meinen Schwestern in Japan für die Ermöglichung meines Studiums und die vorbehaltlose Unterstützung in allen Lebenslagen. 